Hans-Joachim Hauser

\title{
Verteilungswirkungen der Staatsverschuldung
}

Eine kreislauftheoretische Inzidenzbetrachtung 


\section{Hans-Joachim Hauser}

\section{Verteilungswirkungen der Staatsverschuldung}

Unter Zugrundelegung des kreislauftheoretischen Erklärungsansatzes werden die Verteilungswirkungen interpersoneller, intertemporaler und intergenerativer Art untersucht, die von einer staatlichen Kreditnahme ausgehen. Parallel dazu verläuft eine Auseinandersetzung mit den Aussagen der klassischen Schuldtheorie zu diesem Problemkreis.

Hans-Joachim Hauser, geboren 1948 in Mannheim, studierte von 1967-1972 Volkswirtschaftslehre an der Albert-Ludwigs-Universität Freiburg. Er war dort am Institut für Finanzwissenschaft von 1973-1978 als wissenschaftlicher Mitarbeiter tätig. 
Verteilungswirkungen der Staatsverschuldung 


\section{Finanzwissenschaftliche Schriften}

Herausgegeben

von den Professoren

Albers, Krause-Junk, Littmann, Oberhauser, Pohmer, Schmidt

\section{Band 11}

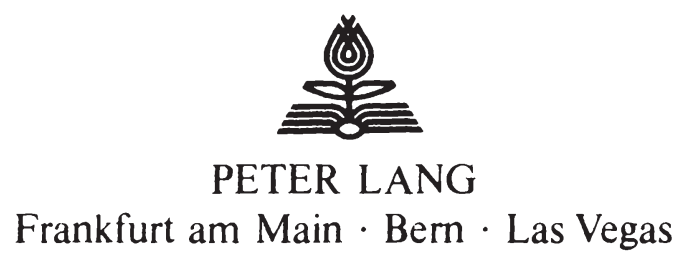




\section{Hans-Joachim Hauser}

\section{Verteilungswirkungen der Staatsverschuldung}

Eine kreislauftheoretische Inzidenzbetrachtung

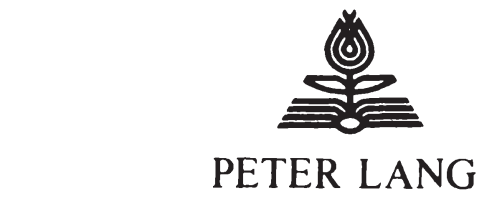

Frankfurt am Main · Bern · Las Vegas 
CIP-Kurztitelaufnahme der Deutschen Bibliothek

\section{Hauser, Hans-Joachim}

Verteilungswirkungen der Staatsverschuldung:

e. kreislauftheoret. Inzidenzbetrachtung/Hans-Joachim

Hauser. - Frankfurt am Main, Bern, Las Vegas:

Lang, 1979.

(Finanzwissenschaftliche Schriften; Bd. 11)

ISBN 3-8204-6341-0

Open Access: The online version of this publication is published on www.peterlang.com and www.econstor.eu under the international Creative Commons License CC-BY 4.0. Learn more on how you can use and share this work: http://creativecommons.org/licenses/by/4.0.

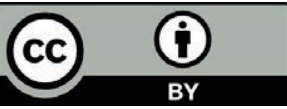

This book is available Open Access thanks to the kind support of ZBW - Leibniz-Informationszentrum Wirtschaft.

ISBN 3-8204-6341-O

ISBN 978-3-631-75249-4 (eBook)

(C) Verlag Peter Lang GmbH, Frankfurt am Main 1979

Alle Rechte vorbehalten.

Nachdruck oder Vervielfaltigung, auch auszugsweise, in allen Formen wie Mikrofilm, Xerographie, Mikrofiche, Mikrocard, Oftset verboten.

Druck: Fotokop Wilhelm Weihert KG, Darmstadt

Titelsatz: Fotosatz Aragall GmbH, Wolfsgangstraße 92, Frankfurt am Main 
I. EINLEITUNG $\ldots \ldots \ldots \ldots \ldots \ldots \ldots \ldots \ldots \ldots \ldots \ldots \ldots$

A Problemstellung $\ldots \ldots \ldots \ldots \ldots \ldots \ldots \ldots \ldots \ldots \ldots \ldots$

B Zielsetzung und Plan der Arbeit .................. 11

II. ENTWICKLUNG DES INZIDENZKONZEPTS $\ldots \ldots \ldots \ldots \ldots \ldots \ldots$

A Untersuchungsobjekt und Methode ................... 13

1. Staatliche Ressourcenbeanspruchung durch Kreditauf-

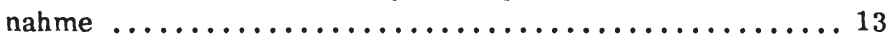

2. Alternative makroökonomische Wirkungs mechanismen .... 14

3. Verteilungs theoretische Vor überlegung ............ 14

4. Indikatoren der Verteilung $\ldots \ldots \ldots \ldots \ldots \ldots \ldots \ldots \ldots \ldots$

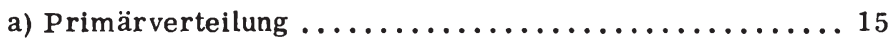

b) Sekundärverteilung $\ldots \ldots \ldots \ldots \ldots \ldots \ldots \ldots \ldots \ldots \ldots$

c) Absolut - und Quotenbetrachtung ............. 16

B Inzidenzanalyse ......................... 17

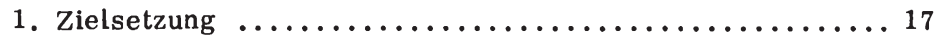

2. Inzidenz im klassischen System .............. 17

3. Inzidenz im kompensatorischen System .......... 18

4. Inzidenzkonzepte ........................ 19

a) effektive Inzidenz $\ldots \ldots \ldots \ldots \ldots \ldots \ldots \ldots \ldots \ldots$

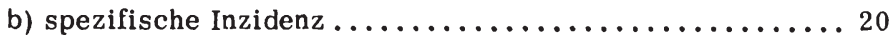

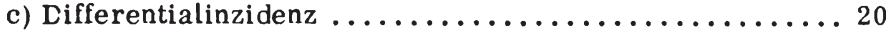

d) Budgetinzidenz $\ldots \ldots \ldots \ldots \ldots \ldots \ldots \ldots \ldots \ldots \ldots \ldots \ldots$

5. Inzidenz der Staatsverschuldung $\ldots \ldots \ldots \ldots \ldots \ldots \ldots \ldots \ldots$

III. VERTEILUNGSEFFEKTE IN DER PHASE CER SCHULD-

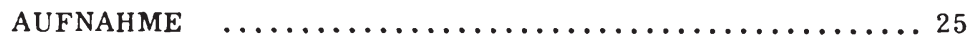

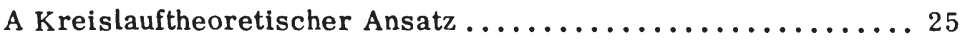

1. Makroökonomische Ausgangsbeziehungen .......... 25

a) Grundkonzept verteilungstheoretischer Interpretation der Kreislaufbeziehungen .................... 25 
b) Keynessche Verteilungsgleichung ............26

c) Funktionale Abhängigkeiten ............... 27

d) Verteilungswirkungen in Abhängigkeit vom Crowding-

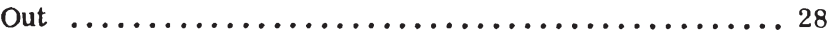

2. Verteilungs modell des Kaldor-Typs ............. 29

a) Grundstruktur des Modells ................. 29

b) Berücksichtigung von verhaltensfunktionen $\ldots \ldots \ldots \ldots 30$

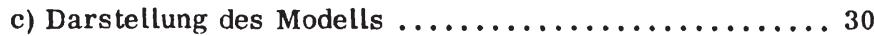

d) Verteilungswirkungen $\ldots \ldots \ldots \ldots \ldots \ldots \ldots \ldots \ldots \ldots \ldots \ldots$

e) Vergleich der Ergebnisse nach Kaldor und der

Keynesschen Verteilungsgleichung ....................

f) Variation der Sparneigungen $\ldots \ldots \ldots \ldots \ldots \ldots \ldots \ldots \ldots$

g) Berücksichtigung eines Crowding-out .......... 35

3. Determinanten der Investitionsnachfrage $\ldots \ldots \ldots \ldots \ldots$

a) Verhältnis von Investitionsneigung zu Investitions -

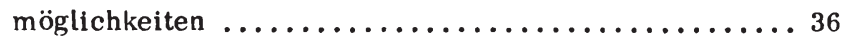

b) Auswirkungen einer staatlichen Schuldaufnahme auf die private Investitionsnachfrage .............. 37

4. Elastizität des Geld- und Kreditangebots ........... 38

a) Rolle des Bankensystems ..................... 38

b) Bestimmungsfaktoren der Elastizität des

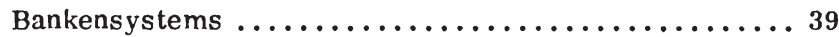

c) Kreditgewährung durch das Bankensystem ........ 40

d) Erfolgsmöglichkeiten kompensierender geldpolitischer

Eingriffe ........................... 41

5. Ergebnis ............................ 42

6. Exkurs: Zu den Verteilungswirkungen der Staats verschuldung in einer Situation der Unterbeschäftigung ... 43

B Neoklassischer Ansatz ...................... 46

1. Charakteristik der Analyse .................46

2. Staatliche Schuldaufnahme als Bestandteil der gesamtwirtschaftlichen Vermögensbildung ............47

3. Entwicklung des Faktorpreises Zins ........... 47

4. Wirkungen auf die funktionale Einkommensverteilung .... 49

5 . Wirkungen auf die institutionelle Verteilung ........ 50

6. Beurteilung der Verteilungsaussagen ........... 51 
IV. VERTEILUNGSEFFEKTE IN DER PHASE DES SCHULDEN -

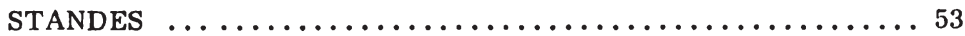

A Auswirkungen der Schuldaufnahme auf die Folgeperiode ..... 53

1. Kreislauftheoretischer Ansatz ................ 53

a) stationäre wirtschaft $\ldots \ldots \ldots \ldots \ldots \ldots \ldots \ldots \ldots \ldots$

b) wachsende Wirtschaft $\ldots \ldots \ldots \ldots \ldots \ldots \ldots \ldots \ldots \ldots$

2. Grenzproduktivitätstheoretischer Ansatz .......... 55

B Zinszahlungen auf Staatspapiere $\ldots \ldots \ldots \ldots \ldots \ldots \ldots$

1. Traditionelle Sicht $\ldots \ldots \ldots \ldots \ldots \ldots \ldots \ldots \ldots \ldots \ldots \ldots \ldots \ldots \ldots$

a) Vorbemerkung $\ldots \ldots \ldots \ldots \ldots \ldots \ldots \ldots \ldots \ldots \ldots$

b) Transfer-Ansatz ..................... 57

c) Methodische Einwände .................. 57

d) Verteilungs relevanz des Steuer-Zins-Kreislaufs für eine kreislauftheoretische Analyse ............ 59

2. Effektive Inzidenz der Zinssteuern ............660

a) Zielsetzung der Analyse .................660

b) Ausgangs modell $\ldots \ldots \ldots \ldots \ldots \ldots \ldots \ldots \ldots \ldots \ldots \ldots \ldots$

c) Verteilungswirkungen bei unterschiedlichen AufbringerEmpfänger-Konstellationen ..................64

c1) Fall I Steuerzahler: Arbeitnehmer

Zinsempfänger: Unternehmer ........664

c2) Fall II Steuerzahler: Unternehmer

Zinsempfänger: Arbeitnehmer .........667

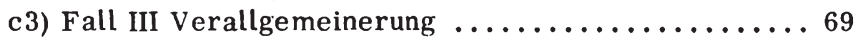

d) Schlußfolgerungen $\ldots \ldots \ldots \ldots \ldots \ldots \ldots \ldots \ldots \ldots$

V. INTERTEMPORALE VERTEILUNGSWIRKUNGEN $\ldots \ldots \ldots \ldots 77$

A Vorüberlegungen $\ldots \ldots \ldots \ldots \ldots \ldots \ldots \ldots \ldots \ldots \ldots \ldots \ldots \ldots$

1. Struktur - und Niveaueffekte $\ldots \ldots \ldots \ldots \ldots \ldots \ldots \ldots$

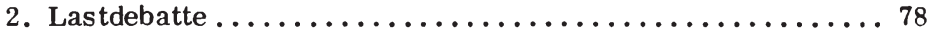

3. Organizistische versus individualistische Staats-

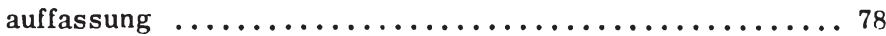

B Makroökonomische Ansätze .................. 80

1. Darstellung des Wachstumsansatzes ............. 80

2. Verteilungspolitische Beurteilung des Wachstumsansatzes . 81

C Mikroökonomische Ansätze .................. 83

1. Ricardo .............................. 83 
a) Äqui valenztheorem $\ldots \ldots \ldots \ldots \ldots \ldots \ldots \ldots \ldots . \ldots . \ldots 3$

b) Gründe für differentielle Effekte $\ldots \ldots \ldots \ldots \ldots \ldots . . \ldots 3$

b1) Mangelnde Information und Illusion ........... 83

b2) Unterschiedliche Zeitpräferenzen ............ 85

c) Verteilungspolitische Beurteilung Ricardos ........ 85

2. Buchanan .............................. 86

a) Grundlegende Thesen $\ldots \ldots \ldots \ldots \ldots \ldots \ldots \ldots \ldots, 86$

b) Kosten im Entscheidungsprozeß.............. 88

c) Kosten der Staatsverschuldung $\ldots \ldots \ldots \ldots \ldots \ldots \ldots, 89$

c1) Entscheidungsbeeinflussende Kosten ........... 89

c2) Entscheidungsbeeinflußte Kosten ............ 89

d) Verteilungspolitische Beurteilung .............. 90

3. Ansätze zur Erklärung intergenerativer Verteilungs effekte ................................ 92

a) Bowen-Davis-Kopf $\ldots \ldots \ldots \ldots \ldots \ldots \ldots \ldots \ldots \ldots, 92$

b) Head $\ldots \ldots \ldots \ldots \ldots \ldots \ldots \ldots \ldots \ldots \ldots \ldots, 93$

D Schlußfolgerungen $\ldots \ldots \ldots \ldots \ldots \ldots \ldots \ldots \ldots \ldots \ldots \ldots, 95$

1. Aussagefähigkeit der mikroökonomischen Ansätze ...... 95

2. Integrierende Betrachtung des mikro- und makroökonomischen Ansatzes ........................ 96

3. Beurteilung in kreislauftheoretischer Sicht ......... 97

E Exkurs: Intertemporale Verteilungseffekte bei einer Ausgangssituation der Unterbeschäftigung - Lastverschiebungsparadox..................... 99

VI. WIRKUNGEN AUF DIE VERMÖGENSVERTEILUNG ........ 101

A Grundlegende Zusammenhänge $\ldots \ldots \ldots \ldots \ldots \ldots \ldots \ldots \ldots$

1. Vermögens verteilung als verteilungspolitisches Problem . 101

2. Vermögenspolitische Begründung einer staatlichen Schuldaufnahme ........................... 101

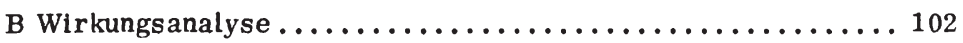

1. Auswirkungen einer staatlichen Neuverschuldung auf die Vermögens verteilung ...................... 102

a) Klassischer Fall ........................ 102

b) Keynesianischer Fall ..................... 103

2. Gläubiger-Schuldner-Hypothese ................... 104

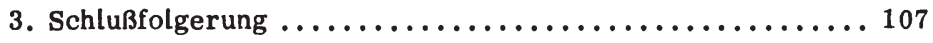

VII. ZUSAMMENFASSUNG DER ERGEBNISSE ............. 109

ANMERKUNGEN $\ldots \ldots \ldots \ldots \ldots \ldots \ldots \ldots \ldots \ldots \ldots \ldots \ldots \ldots \ldots \ldots$

LITERATURVERZEICHNIS .................... 147 


\section{Erstes Kapitel}

\section{E I N L E I T U N G}

\section{A) PROBLEMSTELLUNG}

Von Beginn der Geschichte ökonomischer Lehrmeinungen an war die Theorie des öffentlichen Kredits Gegenstand heftiger Auseinandersetzungen. Brachte man zu Zeiten des klassischen Liberalismus dem Eingreifen des Staates in den Wirtschaftsablauf generell Mißtrauen entgegen, so ist dieses zwar zurückgegangen, doch sind Relikte davon noch heute in der Beurteilung der staatlichen Verschuldung zu finden. Befürchtet man durch eine Staatsverschuldung schon einen nicht kontrollierbaren Zugriff des Staates auf das Sozialprodukt an sich, so geht dies häufig mit dem Vorurteil von der Unproduktivität staatlicher Ausgaben Hand in Hand. Mit dem Anerkennen der konjunkturpolitischen Bedeutung der staatlichen Verschuldung und der damit verbundenen Abkehr von rein objektbezogenen Haushaltsgrundsätzen sind zwar die orthodoxesten Standpunkte aufgeweicht, doch finden sich im politischen wie auch wissenschaftlichen $\mathrm{Be}$ reich immer noch Anklänge an diese traditionelle Einstellung[1]. Nicht zuletzt die erhebliche Zunahme der staatlichen Verschuldung in den letzten Jahren[2] hat die allgemeine Diskussion um die damit verbundenen Wirkungen neu belebt, wobei in der teilweise recht emotional geführten Argumentation immer wieder unterschwellig Vorbehalte gegenüber dem staatlichen Kredit zum Ausdruck gebracht wurden[3].

Im wissenschaftlichen Bereich verläuft die "altehrwürdige"[4] Diskussion um die Wirkungen einer staatlichen Kreditaufnahme zwar nicht mehr so heftig wie noch vor einigen Jahren[5], doch ist die Einschätzung der ökonomischen Effekte dieser Maßnahme keineswegs als einheitlich zu charakterisieren[6]. Sieht man die sowohl in der Alltagsmeinung[7] als auch im politischen Bereich[8] gegenüber diesem Finanzierungsinstrument vorhandenen Animositäten und Vorurteile vor dem Hintergrund einer auch nicht geschlossenen Einschätzung in der finanzwissenschaftlichen Diskussion, so erscheint es von nicht geringem Interesse, die für die widersprüchlichen Einschätzungen des öffentlichen Kredits verantwortlichen Ursachen herauszuarbeiten, um so der Beurteilung eine rationale Basis geben zu können.

Die Gründe für diese Divergenzen sind auf unterschiedlichen Ebenen angesiedelt. Zunächst ist auf den erheblichen Einfluß zu verweisen, der von der Formulierung der Fragestellung ausgeht, kann diese doch entscheidend die Ergebnisse der Untersuchung bestimmen. Damit ist das Problem der Bezugs - oder Vergleichsgröße angesprochen, das in der finanzwissenschaftlichen Diskussion generell, aber ganz speziell in Verbindung mit der Staatsverschuldung eine we- 
sentliche Rolle spielt[9]. In engem Zusammenhang damit steht die äußerst uneinheitliche Terminologie, mit der die einzelnen Autoren die Wirkungen einer staatlichen Schuldaufnahme zu erfassen suchen. Dieser Sachverhalt, der schon als Begriffsverwirrung bezeichnet werden kann, wurde und wird vielfach in der Literatur beklagt[10], man denke dabei nur an die Verwendung des ohnehin schon emotionsträchtigen Begriffs der Last einer Staatsverschuldung mit seinen vielfältigen Interpretationen[11].

Umstrittener dürfte der zweite Begründungskomplex sein, der für die unterschiedlichen Beurteilungen dieses staatlichen Finanzierungsinstruments angeführt werden kann. Es handelt sich um die von den jeweiligen Autoren getroffenen Annahmen bezüglich der makroökonomischen Wirkungsmechanismen, über die der Ressourcentransfer in den staatlichen Bereich abläuft. Allerdings sollte hier eine Konfrontation mit den Vorgängen in der Realität eine Antwort hinsichtlich der komparativen Überlegenheit der einzelnen Ansätze ermöglichen. In Abhängigkeit vom theoretischen Standpunkt werden häufig bestimmte Effekte a priori hinwegdefiniert oder in den Bereich des Vernachlässigbaren verwiesen (dann meist als Sekundäreffekt charakterisiert), die beim Diskussionspartner eine zentrale Rolle spielen mögen[12]. Wird in der Diskussion nur noch auf Ergebnisse abgestellt, und ein solches Vorgehen läßi sich nicht nur im politischen Bereich feststellen[13], so sind einander widersprechende Aussagen und Mißverständnisse eine fast zwangsläufige Folge, werden je nach theoretischem Ausgangspunkt doch u. U. völlig verschiedene Sachverhalte untersucht. Daher müssen diese Wirkungsmechanismen bei der Auseinandersetzung mit einzelnen Autoren stärker in den Vordergrund gerückt werden, um so die Bedingtheiten ihrer Aussagen aufzuzeigen. Wenn etwa Buchanan davon ausgeht, $\mathrm{da} \beta$ "the logic of the analysis seems irrefutable within the limits of the model discussed", so ist dies lediglich die an jede Analyse zu stellende Konsistenzbedingung, während die Behauptung, "and this seems to be the only full appropriate model for the purpose at hand"'[14] einen ungleich gewichtigeren Anspruch verkörpert, der aber durch die Fähigkeit, Phänomene der Realität zu erklären, überprüfbar ist.

Nicht zuletzt im Hinblick auf eine wirtschaftspolitische Umsetzung scheint es von Bedeutung, die ökonomischen Wirkungen der staatlichen Kreditaufnahme in einem einheitlichen Bezugsrahmen zu untersuchen. Aufgrund der weitgehend einmütigen Beurteilung des konjunkturpolitischen Einsatzes der Staatsverschuldung soll dessen Problematik hier nicht zur Diskussion stehen. Statt dessen werden, den Charakter des staatlichen Einnahmeninstruments betonend, die Verteilungseffekte der staatlichen Kreditaufnahme untersucht. Dabei wird auf eine Situation der Vollbeschäftigung abgestellt, in der das Verteilungsproblem aufgrund der konkurrierenden Ansprüche an das Sozialprodukt vorrangig ist; zudem wird der unmittelbare Vergleich mit den Aussagen der auf diese Ausgangssituation abstellenden klassischen Schuldtheorie erleichtert[15]. So ist ein nicht geringer Teil der Aufgabe darin zu sehen, eine systematische, die relevanten Verteilungseffekte der Staatsverschuldung umfassende Betrachtung anzustellen, die in der Literatur zu findenden Teilaspekte[16] in diesen Gesamt- 
zusammenhang zu integrieren und damit deren verteilungspolitische Wertung zu ermöglichen. Die Analyse stellt dabei ausschließlich auf - im Vergleich zu den kritisierten Lastbegriffen - semantisch neutrale Verteilungseffekte ab, auch dies sollte zur Versachlichung der Diskussion beitragen können[17].

\section{B) ZIELSETZUNG UND PLAN DER ARBEIT}

Im Mittelpunkt der Arbeit steht also die Untersuchung der Wirkungen einer staatlichen Schuldaufnahme auf die Einkommens- und Vermögensverteilung. Dabei greift die Analyse auf einen speziellen Erklärungsansatz der Verteilung, nämlich den kreislauftheoretischen, zurück, mit dessen Hilfe die Verteilungswirkungen interpersoneller, intertemporaler und intergenerativer Art in einem Gesamtkonzept erfaßt werden sollen. Die Wahl des kreislauftheoretischen Ansatzes zur Erklärung der Verteilungswirkungen leitet sich zum einen aus dem speziellen Untersuchungsobjekt[18], zum anderen aus der Flexibilität dieses Ansatzes ab. So kann eine bewußte Gegenposition zur - die Diskussion beherrschenden - klassischen Schuldtheorie eingenommen werden, es können aber auch die von dieser Theorie unterstellten Wirkungsabläufe[19] einer verteilungspolitischen Würdigung unterzogen werden (wenn auch aus kreislauftheoretischer Sicht). Dieses Vorhaben ist bei der öffentlichen Schuldaufnahme aufgrund der intertemporalen Verflechtung recht komplex. So sind Auswirkungen auf die Verteilungssituation der Ausgangsperiode, die sich in Zusammenhang mit der Verzinsung ergebenden Verteilungseffekte in den Folgeperioden und die Interdependenzen dieser Effekte im Zeitablauf $\mathrm{zu}$ beachten. Will man das Bild von den Verteilungseffekten abrunden, so muß sich auch noch eine vermögenspolitische Würdigung anschließen. Die Analyse wird sich auf diese Verteilungseffekte beschränken, die gleichfalls verteilungsrelevante Tilgung soll außer Betracht bleiben.

Die Wirkungen auf die Einkommensverteilung werden in zeitlicher Differenzierung zunächst für die Phase der Schuldaufnahme untersucht. Gerade diesem Problemkreis wurde lange Zeit keine Beachtung geschenkt, was - wie noch zu zeigen sein wird - mit der Behandlung innerhalb der klassischen Schuldtheorie zu erklären ist[20]. In diesem Abschnitt wird auch der der klassischen Schuldtheorie entsprechende verteilungstheoretische Ansatz, nämlich der grenzproduktivitäts theoretische, aufgegriffen, um zumindest ein Stück weit den Weg einzuschlagen, den eine verteilungstheoretisch fundierte klassische Schuldtheorie beschreiten müßte.

Dann gilt es, die Verteilungseffekte in der Folgeperiode stellvertretend für alle weiteren Perioden $\mathrm{zu}$ untersuchen. Dabei ist zunächst einmal zu uiberprüfen, ob und wie die Verteilungswirkungen der Schuldaufnahmephase nachwirken. Als zusätzlicher verteilungsrelevanter Sachverhalt, der mit der staatlichen Schuldaufnahme als kausal verbunden anzusehen ist, muß die Besteuerung zur Finanzierung der Zinszahlungen berücksichtigt werden. Dem kreislauftheoreti- 
schen Ansatz entsprechend sind die Auswirkungen der Steuererhebung zur Finanzierung der Zinszahlungen auf die gesamtwirtschaftliche Nachfrage und damit die Einkommensverteilung zu untersuchen.

Die Überlegungen $\mathrm{zu}$ den Verteilungseffekten in der ersten Periode und einer repräsentativen Verzinsungsperiode sowie deren mögliche Interdependenzen liefern dann den Hintergrund für eine Beurteilung der intertemporalen und intergenerativen Effekte, die in der Diskussion um die Wirkungen der Staatsverschuldung einen zentralen Platz einnehmen[21]. Durch die Rückführung auf verteilungstheoretisch relevante Kategorien soll erreicht werden, die unterschiedlichen mikro- und makroökonomischen Ansätze vergleichbar zu machen.

Mit der chronologisch differenzierenden Betrachtung der Einkommensverteilung sind noch nicht alle Verteilungsaspekte hinreichend erfaßt, da auch die Rückwirkungen auf die Vermögensverteilung berücksichtigt werden müssen. Die staatliche Schuldaufnahme stellt ex definitione einen Bestandteil der privaten Vermögensbildung der betreffenden Periode dar. Zudem können sich, wie noch zu zeigen sein wird, infolge einer staatlichen Kreditaufnahme inflationäre Impulse ergeben, was Rückwirkungen auf die Vermögensbestände in Form von Wertveränderungen hat. Auch diesen Aspekt gilt es bei der vermögenspolitischen Beurteilung zu berücksichtigen.

In Analogie zur Behandlung der Verteilungswirkungen anderer staatlicher Einnahmeninstrumente soll hier auf die Inzidenz der Staatsverschuldung abgestellt werden. Die Bedeutung der konkreten Fragestellung wurde oben schon hervorgehoben, weswegen im folgenden Kapitel zuerst der Entwicklung einer dem hier verwendeten theoretischen Ansatz adäquaten Fragestellung nachgegangen werden soll. 


\author{
Zweites Kapitel
}

ENTWICKLUNG DES INZIDENZKONZEPTS

\title{
A) UNTERSUCHUNGSOBJEKT UND METHODE
}

1. Staatliche Ressourcenbeanspruchung durch Kreditaufn ahme

Jeder von staatlichem Einnahme- oder Ausgabeverhalten ausgehende Impuls zieht eine Kette von Reaktionen nach sich, die in unterschiedlicher Form und Intensität auf die einzelnen Wirtschaftssubjekte ausstrahlen. Insofern ist es für den Träger staatlicher Wirtschaftspolitik unabdingbar, diese Wirkungs kette zumindest in ihren qualitativen Zusammenhängen $\mathrm{zu}$ überschauen, da nur so die Verfolgung einer rationalen Wirtschaftspolitik im Sinne von Ziel-MittelSystemen möglich ist. Eines der vorrangigen Ziele stellt dabei das der Verteilung dar, das mit budgetpolitischen Maßnahmen gleich zweifach angesprochen wird, da sowohl mit der Einnahmen- als auch mit der Ausgabenseite[22] diese Zielsetzung verfolgt werden kann, wobei hier allerdings nur erstere behandelt werden soll. Ausgangspunkt der Verteilungsüberlegungen soll der Umstand staatlicher Ressourcenbeanspruchung sein, wodurch - in einer Situation der Vollbeschäftigung - eine konkurrierende Beziehung zwischen staatlichem und privatem Bereich entsteht[23].

Entsprechend ihren spezifischen Eigenschaften sind bei den verschiedenen Finanzierungsarten staatlicher Ausgaben unterschiedliche Verteilungseffekte im privaten Bereich zu erwarten. Mit dieser Betrachtung verläßt man die einfache Sicht der Dichotomie zwischen privatem und öffentlichem Sektor, um die primär interessierenden Verteilungseffekte struktureller Art innerhalb des privanten Bereichs zu erfassen.

Das Untersuchungsobjekt wird im folgenden die staatliche Kreditaufnahme sein. Wir werden uns dabei ausschließlich mit der Schuldaufnahme einer zentralen Behörde im Inland befassen; Sonderprobleme der kommunalen Verschuldung oder der Schuldaufnahme im Ausland bleiben ausgeschlossen. Zunächst soll auch keine nähere Spezifizierung der staatlichen Kreditaufnahme erfolgen, sondern diese Finanzierungsart gewissermaßen e contrario so definiert werden, daß hierbei nicht - wie bei einer Steuerfinanzierung - qua hoheitlicher Macht auf die Verfügungseinkommen der privaten Wirtschaftssubjekte zurückgegriffen wird[24]. Eine erste Präzisierung ist lediglich darin zu sehen, daß auch eine Verausgabung der Mittel unterstellt wird. Diese sehr offene Begriffsfassung hat den Vorteil, daß nicht schon im Stadium der definitorischen Abgrenzung einige Fälle von der Wirkungsanalyse ausgeschlossen werden[25]. 
Um zu Aussagen uiber die strukturellen Verteilungswirkungen eines bestimmten staatlichen Finanzierungsinstruments zu kommen, muß man Einblick in die spezifischen Abläufe gewinnen, die mit dem Ressourcentransfer in den staatlichen Bereich verbunden sind. Im folgenden soll, was diese Wirkungszusammenhänge angeht, zunächst auf zwei konträre, "idealtypische" Modellvorstellungen zurückgegriffen werden.

Zur gütermäßigen Alimentierung einer zusätzlichen Nachfrage des Staates müssen c.p. die privaten Ansprüche in entsprechendem Umfange zurückgedrängt werden[26], was zu Lasten der privaten Konsum- oder Investitionsgüter gehen kann. Der erste Fall, ein Zurückdrängen des privaten Konsumgüteranteils, entspräche Keynesianischen Vorstellungen, wonach sich autonome Ansprüche an das Sozialprodukt - bei noch im einzelnen darzustellenden Voraus setzungen - durchsetzen werden. Neben der privaten Investitionsgüternachfrage stellt eine staatliche, kreditfinanzierte Nachfrage ebenfalls einen autonomen Anspruch dar, so daß die privaten Konsumgüter zurückgedrängt werden müßten.

Die zweite Denkmöglichkeit ist ein klassischen Wirkungsmechanismen gehorchendes System, in dem die zusätzliche staatliche Kreditnachfrage zu Zins steigerungen führt, die ihrerseits den Anteil der privaten Investitionen am Sozialprodukt reduzieren. Dieser Prozeß wird allerdings teilweise durch eine, weil als zinsreagibel angenommen, steigende Ersparnisbildung abgeschwächt. Diese Verdrängungsprozesse sind notwendig, da nach den Vorstellungen dieses Modells die gesamtwirtschaftlichen Ersparnisse die kreditär zu finanzierenden gesamtwirtschaftlichen Ausgaben (d.h. hier die privaten Investitionen und die Staatsausgaben) limitieren.

\section{Verteilungstheoretische Vorüberlegung}

Es soll im folgenden - durchaus bewußt als Alternative zur klassischen Schuldtheorie - die Inzidenz der Staatsverschuldung in kreislauftheoretischer Sicht dargestellt werden. Dabei stellen wir auf die kurzfristigen Verteilungswirkungen $a b$, was den Einbau der staatlichen Verschuldung in ein kreislauftheoretisches Modell erheblich erleichtert. Beide oben angeführten Wirkungsmechanis men sollen berücksichtigt und ihre Konsequenzen auf die Verteilung in kreis lauftheoretischer Sicht dargestellt werden. Die vergleichsweise hohe Flexibilität des kreislauftheoretischen Ansatzes, mit dessen Hilfe verschiedene Formen des Ressourcentransfers in den staatlichen Bereich verteilungstheoretisch interpretierbar sind, stellt eine erste Begründung zur Wahl dieses Konzepts dar. Vor allem aber ist in diesem Zusammenhang auf den Charakter der so finanzierten staatlichen Nachfrage als autonome Größe zu verweisen, die die globalen Nachfrageverhältnisse nicht unerheblich beeinflusst. Schon aus dieser Sicht scheinen die Annahmen des kreislauftheoretischen Ansatzes sinnvoller 
als die einer Welt vollkommener Märkte in der klassischen Theorie. Darüberhinaus vermag der kreislauftheoretische Ansatz unmittelbar Aussagen für verteilungs relevante Untergruppen zu treffen, was sich bei einer Konfrontation mit den Aussagen des grenzproduktivitätstheoretischen Ansatzes als nicht geringer Vorteil erweisen wird. Auch für die Phase des Schuldenstandes schien die kreislauftheoretische Untersuchung sinnvoll, wurde doch auf den Sachverhalt der unterschiedlichen Nachfrageströme je nach Besitzverhältnissen an den staatlichen Papieren schon mehrfach verwiesen. So scheint es naheliegend, für den beispielhaften Fall einer autonomen Nachfrage wie den der kreditfinanzierten Staatsausgaben eine verteilungstheoretische Konzeption zugrundezulegen, die in ihrem Erklärungskalkül auch unmittelbar auf diesen Zusammenhängen aufbaut.

\section{Indikatoren der Verteilung}

\section{a) Primärverteilung}

Jede verteilungstheoretische Aussage muß auf Indikatoren Bezug nehmen, die die Wirkungen einer bestimmten Maßnahme anzeigen. Dies wird bei allen verteilungstheoretischen Ansätzen eine Größe sein, die entweder den prozentualen Anteil einer bestimmten Einkommensart oder aber den Anteil der Einkommen einer bestimmten Gruppe an der Grundgesamtheit (die üblicherweise dem Sozialprodukt entspricht) angibt. Stellt der erste Fall den der funktionellen Einkommensverteilung dar, die die Anteile der dem Produktionsprozeß entstammenden Faktorentgelte angibt, so handelt es sich beim zweiten Fall um einen Ansatz zur Erklärung der personellen Einkommensverteilung, die die Möglichkeit des Bezugs mehrerer Einkommensarten komplex umfaßt. Dieses Phänomen wird auch mit dem Begriff der Querverteilung[27] oder institutionellen[28] Verteilung umschrieben. Welche Einkommenskategorien im einzelnen erklärt werden, hängt entscheidend von dem jeweiligen zur Verwendung gelangenden theoretischen Ansatz ab.

Da den Verteilungspolitiker primär die Verteilungssituation bestimmter Gruppierungen, d.h. die institutionelle Einkommensverteilung interessiert, liefert der hier verwendete kreislauftheoretische Ansatz der Verteilung bereits eine sinnvolle Information. Die die Verteilung determinierenden Parameter beziehen sich auf das Gesamteinkommen einer homogenen (bzw. in ihrem Verhalten als homogen angenommenen) sozioökonomischen Gruppe, so daß dieser Ansatz eine Erklärung der institutionellen Verteilung liefert[29]. Es werden also die Anteile zweier verteilungspolitisch relevanter Gruppierungen bestimmt, wobei sich diese durch bestimmte Verhaltensweisen voneinander abheben. In kreis lauftheoretischen Ansätzen ist dies ublicherweise eine Zweiteilung in die Gruppe der Unternehmer und die der Arbeitnehmer. Die dominante Einkommensart ist bei letzteren kontraktbestimmtes Einkommen, bei den Unternehmern Gewinneinkommen im weiteren Sinne, beide Gruppen können aber auch andere 
Einkommensarten beziehen[30]. Die jeweiligen Anteile des Unternehmer- und Arbeitnehmereinkommens am Sozialprodukt stellen die Primärverteilung dar.

\section{b) Sekundärverteilung}

Durch die Einbeziehung weiterer Aktivitäten des Staates kommt eln zusätzlicher Faktor für die Verteilungseffekte ins Spiel, der über die oben geschilderte Beeinflussung der Primärverteilung hinausgeht[31]. Der Staat erhebt einerseits Steuern und andere Abgaben, durch die das ursprüngliche Bruttoeinkommen der Wirtschaftssubjekte in das verfügbare Einkommen transformiert wird. Andererseits teilt die öffentliche Hand in Gestalt von Transferzahlungen einzelnen Gruppen Einkommensteile zu, die deren verfügbares Einkommen wieder erhöhen. Das verfügbare Einkommen zeigt also an, wie sich die personellen Einkommen der Wirtschaftssubjekte durch die Einwirkung des Staates verändern. Diese Einkommensgröße ist damit ein Indikator für die staatliche Sekundärverteilung[32], die erst das endgültige Verteilungsbild determiniert. Es ist zu betonen, daß dieses Eingreifen des Staates nicht nur Effekte auf die Sekundärverteilung hat, sondern die Primärverteilung und die Sekundärverteilung in hohem Grade interdependent sind[33]. Gerade diese gegenseitigen Abhängigkeiten, denen im Rahmen der üblichen formalen Inzidenzbetrachtungen wenig Beachtung geschenkt wird, werden noch Gegenstand näherer Betrachtung sein.

\section{c) Absolut - und Quotenbetrachtung}

Bei den meisten Verteilungsparametern handelt es sich um Relativzahlen, die den Anteil einer Gruppe zum gesamten Verteilungspotential in Beziehung setzen. Dies stellt eine zulängliche Beschreibung der Verteilungsstruktur dar, was oben als eines der Untersuchungsziele genannt wurde. Dennoch sei schon an dieser Stelle vor einer Verabsolutierung der Quotenaussagen gewarnt, da neben der Verteilungsstruktur dem Verteilungsniveau eine ebensolche Bedeutung als Wohlstandsindikator zukommt.

Dieser Vorbehalt ist vor allem aufgrund der durch den Untersuchungsgegenstand bedingten zeitübergreifenden Sicht angebracht, eine ausschließliche $\mathrm{Be}$ trachtung der Strukturentwicklung würde ein nur unvollständiges Verteilungs bild geben[34][35]. So werden zunächst in der Schuldaufnahmephase bei Untersuchung der strukturellen Verteilung die Quotenaussagen im Vordergrund stehen, während bei den Fragen der intertemporalen Verteilung zusätzlich die Verteilungsniveaus in die Beurteilung einbezogen werden müssen. 


\section{B) INZIDENZANALYSE}

\section{Zielsetzung}

Allgemein läßt sich als Zielsetzung einer Inzidenzbetrachtung festhalten, daß sie die sich aufgrund eines staatlichen Eingriffs ergebenden Verteilungseffekte für den privaten Bereich aufzeigen will[36]. Dabei handelt es sich um eine recht offene Definition, die auf alle staatlichen Maßnahmen angewendet werden kann. Die weitere inhaltliche Präzisierung des Inzidenzbegriffs muß sich seinem teleologischen Charakter entsprechend[37] nach der Fragestellung und dem zur Verwendung kommenden theoretischen Ansatz richten; so wird in dieser Untersuchung nur auf makroökonomische Inzidenzüberlegungen abgestellt werden. Auch Musgrave, der die Entwicklung der Inzidenztheorie entscheidend geprägt hat, weist auf diese Bedingtheiten hin. Zwar führt er seine Inzidenzanalysen primär im Rahmen der neoklassischen Theorie durch[38], stellt aber einige ergänzende Überlegungen zur Inzidenz in einem kompensatorischen System an, das dem von uns als keynesianisch bezeichneten entspricht, und kommt hierfür auch zu anderen Inhalten[39], auf die noch einzugehen sein wird.

Es soll zunächst die neoklassische Inzidenzkonzeption dargestellt, im Anschluß daran der unserer kreislauftheoretisch ausgerichteten Analyse adäquate Ansatz aufgezeigt werden.

\section{Inzidenz im klassischen system}

Angesichts der Vielzahl möglicher Effekte, die mit einer staatlichen Maßnahme verbunden sein können, ist es vorrangige Aufgabe jeglicher Inzidenzanalyse, die zu untersuchenden Wirkungen klar abzugrenzen. So zeigen sich die wesentlichen Unterschiede der dogmengeschichtlich weit zurückreichenden Diskussion vor allem darin, welche Effekte aus der Gesamtheit der mit einer Steuerauflage[40] verbundenen aufgegriffen werden[41]. Wenn diese Diskussion auch nicht als abgeschlossen betrachtet werden kann, lassen sich doch gewisse Gemeinsamkeiten herausstellen. Das heute in der Literatur akzeptierte, konstitutive Element einer Inzidenzanalyse geht auf Musgrave zurück, der die Änderung der Verteilung des für den privaten Gebrauch zur Verfügung stehenden Einkommens als Inzidenz einer finanzpolitischen Maßnahme definiert[42]. Im Mittelpunkt einer Inzidenzanalyse stehen also die Verteilungseffekte. Explizit ausgeschlossen bleiben hiernach alle Wirkungen auf das Gesamteinkommen und die Produktion, die mit der betrachteten Maßnahme verbunden sein mögen. Gleiches gilt für die Effekte, die sich durch den Ressourcentransfer ergeben, da diese als Folge der Verausgabung, nicht aber der Einnahmenerzielung angesehen werden. Zwar sieht Musgrave die Interdependenzen zwischen diesen verschiedenen Wirkungen, schließt sie aber dennoch von der Inzidenzbetrachtung im engeren Sinne aus[43]. 
Da auch die makroökonomischen Inzidenzanalysen zumeist in klassischen Wirkungsmechanismen gehorchenden Modellen abgehandelt werden, was ihrer Herkunft von mikroökonomischen, preistheoretischen Ansätzen entspricht, ist es nur folgerichtig, daß zur Ermittlung der Verteilungseffekte auf die Veränderungen der relativen Güter-bzw. Faktorpreise abgestellt wird. Lediglich diese erscheinen als sinnvoller Indikator für Verteilungsaussagen; die Entwicklung der absoluten Preise und damit des Preisniveaus hat für überlegungen im walras-paretianischen Rahmen keine Bedeutung[44]. Dies betont auch McLure: "The knowledge of the changes in absolute prices, per se, is not essential for the analysis of tax incidence, at any rate. As we have seen tax incidence is primarily a matter of shifts in relative prices. Redistribution of income can result from inflationary or deflationary policies, including those of taxation. But these are best considered as separate phenomena resulting from the chosen macroeconomic policy rather than from the particular tax in question"'[45]. So erkennt zwar McLure durchaus die Kausalitätsbeziehung zwischen möglichen inflationären Effekten und steuerlichen Maßnahmen an, will diese aber dennoch von der Inzidenzanalyse ausschließen.

Solange ein System auf exogene Impulse mit entsprechender Flexibilität der Preise auf allen Güter- und Faktormärkten bei Wahrung der Vollbeschäftigung reagiert, sind für verteilungspolitische Aussagen tatsächlich nur die relativen Preise von Belang. Ist das Funktionieren dieser Mechanismen in der Realität aber nicht mehr in idealtypischer Weise gegeben, haben wir es also mit einem im Musgraveschen Sinne kompensatorischen System zu tun, so kommen Veränderungen des Preisniveaus gerade für Aussagen über die Verteilung erhebliche Bedeutung zu. Für die kreislauftheoretische Betrachtungsweise der Verteilung sind diese Preisniveauveränderungen die entscheidenden Mechanismen, mit denen das System auf exogene Störungen reagiert. Es wird bei den Überlegungen zur Inzidenz im kompensatorischen System zu prüfen sein, inwieweit diese grundlegenden Unterschiede in den Wirkungsmechanismen auch im jeweiligen Inzidenzkonzept Berücksichtigung finden.

\section{Inzidenz im kompensatorischen System}

Gegenüber dem neoklassischer Theorie verbundenen Inzidenzansatz, der die über Veränderungen relativer Preise eintretenden Verteilungseffekte im totalen Gleichgewicht bis auf die einzelnen Märkte zurückverfolgt, muß der Ansatz einer kreislauftheoretisch orientierten Inzidenzanalyse globaler sein. Wenn auch bei jeder makroökonomischen Inzidenzuntersuchung die Gefahr besteht, daß die Aussagefähigkeit durch Aggregation erheblich reduziert wird[46], muß dieser Schritt dennoch als Annäherung an die Realität gewagt werden.

Auch Musgrave entwickelt, wie schon erwähnt, ein Inzidenzkonzept für das kompensatorische System. Im Unterschied zum klassischen Ansatz, der ex definitione eine Situation der Vollbeschäftigung garantiert, sind hier Einkommens - und Beschäftigungswirkungen sowie Preisniveaueffekte $\mathrm{zu}$ erwarten. 
Wiederum soll aber die Inzidenz diese Beschäftigungs - und Preiseffekte nicht enthalten, was nach Musgrave die Einbeziehung staatlicher Stabilisierungsmaßnahmen wie etwa monetärer Restriktionen notwendig macht, um so reine Verteilungseffekte ausweisen zu können[47]. Es wird der Geldpolitik eine kompensierende Rolle zugeschrieben[48], bestimmte Wirkungsabläufe (nämlich solche mit preissteigernden Impulsen) sind a priori von der Verteilungsanalyse ausgeschlossen. Aufgrund dieser Annahme, die gewissermaßen einen Als-Ob-Zustand der neoklassischen Theorie herstellen soll, kann aber diese Inzidenzkonzeption nicht mehr als realitätsnah bezeichnet werden. Wenn bestimmte Maßnahmen mit Preisniveaueffekten einhergehen, so ist diese unmittelbare Kausalität zu berücksichtigen. Ein auf das kompensatorische System ausgerichtetes Inzidenzkonzept muß die von Preisniveauvariationen ausgehenden Verteilungswirkungen enthalten[49], also auch die Inzidenz der Inflation ausweisen, was in den Ansätzen nach Musgrave vermieden wird. Werden bestimmte, mit einer Maßnahme einhergehende Wirkungen ausgeschlossen, kann eine solche Untersuchung nicht mehr das gesamte in der Realität zu beobachtende Spektrum an Wirkungsabläufen erfassen. Eine solche Inzidenz weist statt dessen die Effekte der finanzpolitischen Maßnahme und die kompensierender geldpolitischer Effekte aus[50]. Dieses Vorgehen impliziert zum einen, daß eine solche Kompensation durch die Geldpolitik uiberhaupt möglich ist, und zum anderen, daß sie auch von den entsprechenden wirtschaftspolitischen Instanzen durchgefürt wird.

Nach diesen grundsätzlichen Vorïberlegungen zur Inzidenz im keynesianischen System wollen wir uns im folgenden mit der die konkrete Fragestellung betreffenden Inzidenzform befassen.

\section{Inzidenzkonzepte}

\section{a) Effektive Inzidenz}

Wenn als Ziel einer Inzidenzanalyse die Erfassung der Verteilungseffekte einer finanzpolitischen Maßnahme genannt wurde, kann es letztlich nur darum gehen, die endgültigen Verteilungsänderungen nach Ablauf aller Anpassungsprozesse zu ermitteln, die als die tatsächliche oder effektive Inzidenz bezeichnet wird[51]. Die vielfach geforderte verteilungstheoretische Fundierung[52] bedeutet dabei bereits einen wichtigen Schritt auf dem Wege zu einer gehaltvollen Inzidenzanalyse, da nur so die Einseitigkeiten partialanalytischer Betrachtungen überwunden werden können.

Neben dieser effektiven Inzidenz verlieren andere Inzidenzaussagen inhaltlich an Bedeutung. Bestenfalls Maßstabfunktion[53] für die Zielerreichung bei wirtschaftspolitischen Entscheidungen kommt der Aussage der beabsichtigten oder formalen Inzidenz zu, die Ausdruck der vom Gesetzgeber intendierten Belastungen ist. Ähnliches gilt für die gesetzliche Inzidenz (impact bzw. statutory incidence), die an den äußerlichen Merkmalen der Zahlungsvorgänge an- 
knüpft[54] und nicht zuletzt deswegen häufig vorschnell mit der effektiven Inzidenz gleichgesetzt wird.

Damit sind rein formale Unterscheidungen zwischen unterschiedlichen Inzidenzformen eingefüht, die aber im Zusammenhang mit wirkungsanalytischen Überlegungen nur sekundär sein können, da es hierbei immer um die Frage nach der effektiven Inzidenz geht. Für eine Wirkungsanalyse wesentlich sind dagegen die unterschiedlichen Fragestellungen, die mit der in finanzwissenschaftlichen Untersuchungen gängigen Unterscheidung in spezifische Inzidenz, Differentialinzidenz und Budgetinzidenz verbunden sind. Es sollen im folgenden diese unterschiedlichen Inzidenzkonzepte im Hinblick auf die Eignung für die vorliegende Problemstellung verglichen werden.

\section{b) Spezifische Inzidenz}

Das engste der eingeführten Konzepte ist das der spezifischen Inzidenz, wonach die Inzidenzeffekte bei jeweils isolierter Variation der Einnahmen- oder aber Ausgabenseite untersucht werden. In Musgravescher Terminologie werden dabei entweder die Staatsausgaben (bei der spezifischen Einnahmeninzidenz) oder aber die Staatseinnahmen (bei der spezifischen Ausgabeninzidenz) als konstant angenommen, so daß sich je nach Änderungsrichtung der Variablen bei Vollbeschäftigung als Ausgangssituation inflationäre oder deflationäre Effekte einstellen werden. Gerade die Preisniveaubewegungen, die aus allen Inzidenzüberlegungen verbannt werden, veranlaßten Musgrave, dieses Konzept zu verwerfen.

In kreislauftheoretischer Sicht sind jedoch nicht die Preiseffekte die eigentliche Beschränkung dieses Ansatzes, sondern, stellt man auf die spezifische Einnahmeninzidenz $a b$, die (zu) restriktive Annahme bezüglich der Ausgabenseite. Geht man hypothetisch von einer Einnahmenerhöhung aus, so impliziert die spezifische Inzidenzbetrachtung die Stillegung der Mittel, eine, wenn auch etwa in der Bundes republik institutionell verankerte, so doch wenig repräsentative Verwendungsart[55]. Die Untersuchungsform der spezifischen Inzidenz kann also einer kreislauftheoretischen Betrachtung, für die die Art der Verwendung der Einnahmen eine die Wirkungen determinierende Rolle spielt, nicht zugrunde gelegt werden[56].

\section{c) Differentialinzidenz}

Dieses Konzept vergleicht die Verteilungswirkungen beim Austausch zweier aufkommensgleicher Maßnahmen, wobei je nach Definition dieser Aufkommensgleichheit (nominal oder real) unterschiedliche Ergebnisse zu erwarten sind[57].

Geht dieses Konzept in realer Betrachtung von einer Aufkommensgleichheit aus, so müßten die Verteilungseffekte eigentlich schon bekannt sein[58]. Die einfacher vorzustellende nominale Gleichheit des Aufkommens hätte zur Folge, 
daß die staatlichen Realausgaben nicht mehr determiniert sind bzw. eine zusätzliche Überschuß- oder Defizitfinanzierung notwendig wird, um das reale Ausgabenniveau aufrechtzuerhalten. Damit können aber wieder Preisniveaueffekte einhergehen, weswegen die Differentialinzidenz zumeist zwei Maßnahmen untersucht, die ein gleiches Realaufkommen erbringen.

Unabhängig von diesen Einwänden bleibt das Problem, daß die Wirkungen zweier Maßnahmen erfaßt werden müssen, so daß sich die Frage stellt, ob nicht nur kasuistische Aussagen möglich sind, da nach kombinatorischen $\mathrm{Ge}-$ setzen alle denkbaren Finanzierungsmaßnahmen verglichen werden könnten. Um dem letztgenannten Einwand zu begegnen, wird auf eine verteilungsneutrale Steuer als gemeinsamer Nenner zurückgegriffen. Dies stellt zwar eine Vereinfachung dar, es erhebt sich aber immer noch die Frage, wie die Wirkungen der Einzelkomponenten erfaßt werden können[59], ohne - um mit Fecher zu sprechen - einem infiniten Regreß zu verfallen[60].

\section{d) Budgetinzidenz}

Dieses Konzept versucht eine sehr umfassende Problemstellung zu lösen, da die Einnahmen- und die Ausgabenseite des Budgets mit ihren Verteilungswirkungen erfaßt werden sollen. Sowohl für das klassische wie auch für das kompensatorische System wird die Bedeutung dieser Problemstellung nicht in Zweifel gezogen. So sind auch die Haupteinwände meist pragmatischer Art, wenden sich also nicht prinzipiell gegen die Methodik. Bei diesem Untersuchungsziel tritt das Problem der Zurechnung gleich in zweifacher Form auf. Einerseits wird das Prinzip der Nonaffektation durchbrochen, d.h., bestimmten Ausgaben müssen Einnahmen zugeordnet werden, andererseits muß eine Zurechnung der Verteilungswirkungen staatlicher Leistungen auf verteilungsrelevante Gruppierungen erfolgen. Für eine verteilungspolitische Beurteilung stellt sich zusätzlich das Bewertungsproblem dieser staatlichen Leistungen.

Wenn es sich bei dieser Problemstellung der saldierenden Budgetbetrachtung auch um eine relevante, wirtschaftspolitische Frage handelt, so soll hier doch nur auf die Verteilungseffekte der Finanzierungsseite abgestellt werden[61], da wir die Wirkungen der staatlichen Schuldaufnahme in allgemeiner Form darzustellen versuchen. Diese Allgemeinheit der Aussage ist aber bei dem oben geschilderten Konzept der Budgetinzidenz nicht gewährleistet, da diese ein bestimmtes, einmaliges Budget im Hinblick auf seine Verteilungswirkungen beurteilt[62]. Unser Untersuchungsziel ist insofern dieser komplexeren Fragestellung vorgelagert, als zur Beantwortung der Frage nach der Budgetinzidenz auch die Verteilungswirkungen der Finanzierungsseite bekannt sein müssen. Dies setzt allerdings voraus, daß die Verteilungseffekte einer bestimmten Finanzierungsart unabhängig von der konkreten Gestalt des Ausgabenprogramms zu erfassen sind. Hiermit ist ein Aggregationsproblem angesprochen, da sich bei hinreichend disaggregierender Betrachtung durchaus unterschiedliche Verteilungseffekte für die einzelnen Wirtschaftssubjekte in Abhängigkeit vom konkre- 
ten Ausgabenprogramm ergeben können[63]. Für den unserer Analyse zugrunde gelegten vertellungstheoretischen Ansatz spielt eine Differenzierung der Güterkäufe des Staates allerdings keine entscheidende Rolle, da in dem hochaggregierten Modell lediglich auf die Entwicklung der globalen Nachfrageströme abzustellen ist. Allerdings impliziert diese Sicht eine gewisse Flexibilität der Produktionsstruktur[64]; unbeschadet davon werden auch strukturelle Effekte eintreten, die dann aber in der Durchschnittsbetrachtung des Aggregats untergehen.

Eine isolierte Betrachtung der Verteilungswirkungen der Einnahmenseite des Budgets ist in kreislauftheoretischer Sicht möglich, damit kann der oben geschilderte Teilaspekt der Fragestellung der Budgetinzidenz zureichend beantwortet werden. Bei dieser Budgetinzidenz, die lediglich die Verausgabung der Mittel, nicht aber die distributiven Aspekte der damit finanzierten Projekte erfaßt, tritt dann auch das Problem der Nonaffektation in den Hintergrund.

\section{Inzidenz der statsverschuldung}

Angesichts der Vielfältigkeit des Inzidenzbegriffs stellt sich die Frage, inwieweit die Verwendung eines Inzidenzkonzepts für die Staatsverschuldung überhaupt sinnvoll ist. Zunächst ließe sich rein pragmatisch argumentieren, daß dieser Versuch in der Literatur immer wieder unternommen wurde[65]. Neben dieser eher vordergründigen Aussage ist aber auf die alle staatlichen Einnahmen- und Ausgabeninstrumente umfassende Bedeutung des Inzidenzbegriffs $z u$ verweisen. Da der Ressourcentransfer vom privaten in den öffentlichen Bereich Opportunitätskosten für die privaten Wirtschaftssubjekte hervorruft, erscheint es durchaus angebracht, diese je nach Finanzierungsart unterschiedlichen Effekte in vergleichbarer Form darzustellen. Gerade die tatsächliche oder vermeintliche Lautlosigkeit einer Finanzierung über Kreditaufnahme spricht für die Ermittlung der Inzidenz der Staatsverschuldung.

Die Untersuchung der Inzidenz der Staatsverschuldung in kreislauftheoretischer Sicht hat darüber hinaus die Zielsetzung, die Grenzen der Aussagefähigkeit der üblicherweise verwendeten Inzidenzkonzepte aufzuzeigen. Durch die Wahl des dem Untersuchungsobjekt adäquaten verteilungstheoretischen Ansatzes mußten auch im Hinblick auf das Inzidenzkonzept gewisse Änderungen eingeführt werden. Dieses erlaubt nun die Erfassung auch solcher Phänomene, die in der eingeführten Inzidenztheorie (und damit auch bei den Überlegungen der klassischen Schuldtheorie) ex definitione nicht in Erscheinung treten können, obwohl sie für die Beurteilung der Verteilungswirkungen der Staatsverschuldung von großer Bedeutung sind.

Nach diesen Vorüberlegungen ist die Fragestellung für die folgende, kreislauftheoretische Untersuchung klargestellt. Es soll die in obigem Sinne definierte Budgetinzidenz der Staatsverschuldung herausgearbeitet werden, die zwar die Verausgabung der Mittel unterstellt, nicht aber die Verteilungseffekte der da- 
mit finanzierten Staatsausgaben berücksichtigt. Zudem stellt die Saldierung der zwei Budgetseiten kein methodisches Problem dar, da die Einzelwirkungen unabhängig voneinander ausgewiesen werden können.

An diesem Punkt ist etwa an Andel zu erinnern, der bei einer verteilungs politischen Beurteilung die Berücksichtigung der jeweils relevanten Alternative fordert[66]. Mit der Aufforderung zu einer solchen Sichtweise will Andel das seiner Ansicht nach (zu) unguinstige Bild von den Verteilungswirkungen der Staatsverschuldung korrigieren[67]. Auch hier kommt das oben schon angesprochene Problem der Bezugsgröße zum Ausdruck, denn es ist selbstverständlich, daß durch Vergleich mit einer verteilungsmäßig ungünstigeren Alternative eine an sich nicht positiv $z u$ beurteilende Maßnahme relativ gewinnt[68]. Allerdings läßt sich durch die Wahl entsprechender Vergleichs größen nahezu jede beliebige Aussage erzielen.

Damit soll das Problem der "relevanten Alternative" in verteilungspolitischer Sicht keinesfalls verleugnet werden, aber auch zur Beantwortung einer solch konkreten Frage ist die Kenntnis der effektiven Inzidenz der zur Diskussion stehenden Finanzierungsmaßnahmen Vorbedingung. So erscheint es insgesamt sinnvoller, die Inzidenzwirkungen der einzelnen Maßnahmen aufzuzeigen, die dann im konkreten Anwendungsfall kombiniert werden können. Dies ermöglicht auch ein differenzierteres Eingehen auf diese Maßnahme, da nicht zu erwarten ist, daß die Wirkungen eindeutig determiniert sind.

Wenn im folgenden eine chronologische Betrachtung vollzogen wird, die die Effekte auf die Primärverteilung in der Schuldaufnahmephase und dann die Effekte auf Primär - und Sekundärverteilung in der "Bestandsphase" unter sucht, so ist dies die Konsequenz unseres Inzidenzkonzepts, das die Verteilungswirkungen einer bestimmten Finanzierungsart verfolgen will. Aufgrund der spezifischen Form der Staatsverschuldung bedingt dies eine (komparativstatische) intertemporale Betrachtungsweise. 

Drittes Kapitel

VERTEILUNGSEFFEKTE IN DER PHASE DER SCHULDAUF-
NAHME

A) KREISLAUFTHEORETISCHER ANSATZ

1. Makroökonomische Ausgangsbeziehungen

a) Grundkonzept verteilungstheoretischer Interpretationen der Kreislaufbeziehungen

Im Mittelpunkt der auf Keynesschen Gedanken basierenden Erklärungsansätze der Verteilung stehen die Faktoren der gesamtwirtschaftlichen Nachfrage, die sowohl für das Niveau als auch für die Struktur der Verteilung entscheidend sind[69]. Ausgangspunkt aller dieser Überlegungen sind die Identitäten der gesamtwirtschaftlichen Kreislaufbeziehungen, aus denen sich Definitionsgleichungen für die Einkommen der Gruppe der Unternehmer einerseits und der Arbeitnehmer andererseits ableiten lassen.

Für die verteilungstheoretische Aussage ist dabei wesentlich, daß im Kreis lauf alle Ausgaben an die Unternehmer zurückfließen. Die kreislauftheoretische Erklärung vermag so die Existenz von Kreislaufgewinnen oder windfall profits aufzuzeigen und zu begründen[70].

Ohne die Bedeutung anderer theoretischer Erklärungsansätze der Verteilung generell in Frage stellen zu wollen, muß man festhalten, daß die Verteilungs wirkungen einer Schuldaufnahme in der Vollbeschäftigung ohne Berücksichtigung der Kreislaufzusammenhänge nicht erklärbar sind, stellt die staatliche Ausgabenfinanzierung über Kreditaufnahme doch eine autonome Ausgabenkategorie dar[71], die unmittelbar die Nachfrageströme beeinflußt. Die Ergebnisse der folgenden kreislauftheoretischen Analyse erheben insofern durchaus den Anspruch auf verteilungspolitische Relevanz.

Als erste formale Darstellung, die auch in Verbindung mit dem Problem der öffentlichen Verschuldung in der Literatur zu finden ist[72], sei auf die sogenannte Keynessche Verteilungsgleichung (oder 2. Keynessche Gleichung)[73] eingegangen. 
b) Keynessche Verteilungsgleichung

Mit den folgenden Definitionsgleichungen sei eine geschlossene Wirtschaft erfaßt, in der sich die Staatstätigkeit auf die über Kreditaufnahme $\left(K_{s t}\right)$ finanzlerten Staatsausgaben $\left(A_{S t}\right)$ beschränkt. Oben wurde schon auf die Vorzüge einer Betrachtungsweise verwiesen, die den Staat mit seinen Aktivitäten neu in das Modell einfuhrt[74]. So kann sich das Interesse auf die Entwicklung der Primärverteilung konzentrieren, da keine Steuererhebung und deren Verausgabung zu berücksichtigen sind, d.h. die Sekundärverteilung noch keine Rolle spielt. Wir gehen von den Definitionsgleichungen aus, die die Entstehung und Verteilung des Sozialprodukts beschreiben[75]:

$$
\begin{array}{ll}
\text { III.1. } & \mathrm{Y}=\mathrm{C}+\mathrm{I}_{\mathrm{pr}}+\mathrm{A}_{\text {st }} \\
\text { III.2. } & \mathrm{Y}=\mathrm{Y}_{\mathrm{u}}+\mathrm{Y}_{\mathrm{nu}}
\end{array}
$$

In der Budgetgleichung des Staates kommt die ausschließlich kreditäre Finanzierungsform zum Ausdruck:

III. 3. $\quad \mathrm{A}_{\text {st }}=\mathrm{K}_{\mathrm{st}}$

Ferner muß dieses System der Bedingung genügen, daß die gesamtwirtschaftliche Ersparnis der Vermögensbildung entspricht. Da wir von einer staatlichen positiven Vermögensbildung absehen, die staatliche Verschuldung aber Bestandteil der privaten Vermögensbildung ist[76], ergibt sich aus dieser Bedingung die modifizierte Form

III.4. $\mathrm{S}=\mathrm{V}_{\mathrm{pr}}=\mathrm{I}_{\mathrm{pr}}+\mathrm{K}_{\mathrm{st}}$

Aus diesen Gleichungen läßt sich nach Unterteilung der Einkommensaggregate der Unternehmer $\left(Y_{u}\right)$ und der Nichtunternehmer $\left(Y_{n u}\right)$ in ihren Konsum-bzw. Ersparnisanteil folgende Beziehung ableiten:

III. 5. $\quad \mathrm{Y}_{\mathrm{u}}=\mathrm{C}_{\mathrm{u}}+\mathrm{I}_{\mathrm{pr}}+\mathrm{K}_{\mathrm{st}}-\mathrm{S}_{\mathrm{nu}}$

Diese Bestimmungsgleichung für das Unternehmereinkommen, als Keynessche Verteilungsgleichung bekannt, veranschaulicht rein definitorische Zusammenhänge, aufgrund ihres tautologischen Charakters kann sie unbeschränkte Gültigkeit beanspruchen. Danach wird das Unternehmereinkommen - bei strenger ceteris-paribus-Argumentation - positiv beeinflußt von den privaten Investitionen und der staatlichen Kreditaufnahme als Elementen der privaten Vermögensbildung sowie dem Konsum der Unternehmerhaushalte, während die Ersparnis der Nichtunternehmer negativ auf die Unternehmereinkommen wirkt. 
Um die Relativität dieser Aussagen aufzuzeigen, sei aus den Definitionsgleichungen auch die analog aufgebaute Bestimmungsgleichung für das NichtUnternehmereinkommen abgeleitet:

III.6. $\mathrm{Y}_{\mathrm{nu}}=\mathrm{C}_{\mathrm{nu}}+\mathrm{I}_{\mathrm{pr}}+\mathrm{K}_{\mathrm{st}}-\mathrm{S}_{\mathrm{u}}$

Aus dem unmittelbaren Vergleich wird ersichtlich, daß noch weitere Annahmen notwendig seln müssen, um aus diesen Gleichungen eindeutige Schlußfolgerungen ziehen zu können. Dazu ist es erforderlich, die Interdependenzen der in den Gleichungen enthaltenen Größen zu erhellen. Hierbei sind die Wirkungsmechanismen bedeutsam, die bei Größenänderungen als wahrscheinlich für die Erklärung der Abläufe anzunehmen sind.

\section{c) Funktionale Abhängigkeiten}

Für die Interpretation dieser Zusammenhänge ist ein wesentliches Systemmerkmal marktwirtschaftlicher Wirtschaftsordnungen ausschlaggebend, das oben schon angesprochen wurde. Die Unternehmer können ihre Ansprüche an das Sozialprodukt autonom durchsetzen, da die hierfür aufzubringenden Ausgaben wieder als Einkommen im Kreislauf an sie zurückfließen. Diese Möglichkeit ist für die Gruppe der Arbeitnehmer nicht gegeben. Berücksichtigt man, daß nicht die Arbeitnehmer, sondern die Unternehmer die über das Ausmaß der Investitionen bestimmende Gruppe sind und daß eine kreditäre finanzierte Ausweitung des Konsums der Arbeitnehmer nicht im Kreislauf zu diesen als Einkommen zurückfließt, so wird die Erklärungsdominanz der Einkommensgleichung III. 5. deutlich.

Neben den autonomen Ansprüchen der Unternehmer enthält das System aufgrund der Einbeziehung des öffentlichen Sektors auch solche des Staates, die sich gleichfalls in der privaten Vermögensbildung niederschlagen. Es ist also ferner zu untersuchen, welche Effekte sich innerhalb dieses Aggregats der Vermögensbildung ergeben. Hierzu kann auf die oben eingeführten alternativen Wirkungsmechanismen zurückgegriffen werden. Während nach der klassischen Hypothese die private Vermögensbildung eine durch das Sparen gegebene Größe darstellt und damit die staatliche Nachfrage mit privaten Investitionen konkurrieren müßte, ergeben sich im keynesianischen System nicht ohne weiteres solche Konkurrenzbeziehungen.

Der Grad der Substitution zwischen privaten Investitionen und staatlichen Ansprüchen, in der Literatur auch als Crowding-out-Effekt bezeichnet[78], wird dabei im klassischen Ansatz von den jeweiligen Zinselastizitäten der Sparund Investitionsfunktionen bestimmt[79]. In jedem Falle ist aber eine entsprechende, freiwillige Ersparnis notwendige Voraussetzung zur Vermögensbildung. Im Falle eines völlig zinsunelastischen Kapitalangebots würde bei dann konstant bleibender privater Vermögensbildung eine zusätzliche staatliche Verschuldung in vollem Umfange ein Crowding-out hervorrufen. 
Als Grundgedanke keynesianischer Wirkungsabläufe kann dagegen die Vorstellung bezeichnet werden, daß die Ersparnisbildung eine eher passive Rolle spielt, die sich den gesamtwirtschaftlichen Erfordernissen der Vermögensbildung aus $\mathrm{I}_{\mathrm{pr}}+\mathrm{K}_{\mathrm{St}}$ (als aktivem Part) anpaßt[80]. Wir werden in unserem auf die Kreislaufzusammenhänge ausgerichteten Ansatz diese beiden Wirkungsmechanismen mit ihren unterschiedlichen Crowding-out-Effekten auf ihre Verteilungswirkungen hin untersuchen. Damit rückt die Frage nach den Bestimmungsfaktoren des Crowding-out in den Mittelpunkt, was uns bei der Überprüfung des Investitionsverhaltens und der Situation auf den monetären Märkten noch ausführlich beschäftigen wird. Diese Überlegungen werden im Anschluß bzw. in Verbindung mit dem verteilungstheoretischen Ansatz Kaldors angestellt. Zuvor aber sollen einige mögliche Verteilungswirkungen anhand der Keynesschen Verteilungsgleichung dargestellt und interpretiert werden.

\section{d) Verteilungswirkungen in Abhängigkeit vom Crowding-out}

Tritt der Staat mit einer zusätzlichen Kreditnachfrage auf, so ergibt sich im idealtypischen keynesianischen Fall eine Zunahme des Aggregats der privaten Vermögensbildung in voller Höhe der staatlichen Kreditnachfrage, da hier kein Crowding-out angenommen wird. In ceteris-paribus Betrachtung erhöht sich im Umfang der zusätzlichen Vermögensbildung das Unternehmereinkommen, was bei Vollbeschäftigung, die unserem Inzidenzkonzept zugrundegelegt wurde, eine entsprechende Einkommenseinbuße der Nichtunternehmer mit sich bringen muß.

Legt man hingegen das klassische Konzept zugrunde, so ist für den Extremfall einer völligen Konstanz der gesamtwirtschaftlichen Ersparnis die Lösung leicht eingängig. Da sich die private Vermögensbildung nicht ändert, die staatlichen Ansprüche also voll zu Lasten der privaten Investitionen gehen müssen, wird sich - wie aus Gleichung III.5. ersichtlich - auch die Primärverteilung nicht ändern.

Diese Aussage kann aber auch für den Fall aufrecht erhalten werden, daß sich das Aggregat der privaten Vermögensbildung ändert. Es sei daran erinnert, daß je nach Zinselastizitäten der Spar- und Investitionsfunktionen eine Veränderung dieser Größe eintreten kann. Diese wird aber von in absoluter Betrachtung kompensierenden Bewegungen der Größen $C_{u}$ respektive $S_{n u}$ begleitet. Da sich in kreislauftheoretischer Sicht eine Erhöhung der freiwilligen Ersparnisse beider Gruppen in gleicher Richtung auf die Einkommensverteilung auswirkt, bleibt in diesem Falle die Primärverteilung generell unbeeinflußt.

Es ergibt sich so bei der Analyse der Kreislaufzusammenhänge unter Zugrundelegung klassischer Wirkungsmechanismen bezüglich des Crowding-out eine Rechtfertigung des Vorgehens der klassischen Schuldtheorie, die die Effekte in der Schuldaufnahmephase vernachlässigt, da keine Verteilungsänderungen zu erwarten sind. Diese Rechtfertigung erfolgt hier allerdings in einer nicht modellimmanenten Betrachtung; dieser Gedanke wird bei einer sich auch der 
klassischen Methodik bedienenden Argumentation wieder aufgegriffen werden.

Mit diesen aus einfachen definitorischen Gleichungen gewonnenen Zusammenhängen sind bereits erste tendenzielle Aussagen über die Verteilungswirkungen staatlicher Verschuldung möglich. Im folgenden sollen nun diese Überlegungen in ein einfaches postkeynesianisches verteilungstheoretisches Modell eingebaut werden, in dessen Rahmen dann auch eine verbale Interpretation der Ablaufprozesse von einem Gleichgewichtszustand zum anderen erfolgen wird.

\section{Verteilungsmodell des Kaldor - Typs}

\section{a) Grundstruktur des Modells}

Die oben schon diskutierten Kreislaufzusammenhänge werden erst in Verbindung mit Verhaltensfunktionen zu einem theoretischen Gerüst, das die Verteilungszusammenhänge unter Kreislaufaspekten zu erklären vermag. Wir wollen uns dabei an die Urform der postkeynesianischen Verteilungstheorie von Kaldor anschließen. Dieser beruft sich bei der Entwicklung seines verteilungstheoretischen Ansatzes explizit auf Keynessche Gedankengänge, indem er die Multiplikatoranalyse, die bei Keynes für Fragen der Beschäftigungspolitik verwendet wurde, für die Verteilungsproblematik nutzbar machen will[81]. Mit dieser Ausrichtung ist schon die Absage an die Bestimmung des Verteilungs niveaus verbunden, dieses wird mit der Annahme der Vollbeschäftigung eines gegebenen Ressourcenbestandes als bekannt vorausgesetzt. Sein Untersuchungsziel ist die strukturelle Verteilung der Einkommen auf die Gruppe der Gewinnbezieher und der Arbeitnehmer.

Der entscheidende Ausgangspunkt für die Wirkungsabläufe innerhalb dieses Modells ist darin zu sehen, daß Abweichungen zwischen notwendig werdendem und freiwillig geleistetem gesamtwirtschaftlichem Konsumverzicht über Verteilungs änderungen ausgeglichen werden. Innerhalb bestimmter Grenzen, auf die noch einzugehen sein wird, paßt sich die Ersparnisbildung der gesamtwirtschaftlichen Investition[82] an, wobei dieser Mechanismus sowohl für expansive als auch kontraktive Bewegungen Gültigkeit beansprucht. In Verbindung mit der Vollbeschäftigungsannahme impliziert diese Sicht, daß es ein Spektrum von Gleichgewichtssituationen bei Vollbeschäftigung gibt, die durch unterschiedliche Einkommensverteilungen gekennzeichnet sind. Zur Bestimmung der Einkommensverteilung ist allerdings noch eine Hypothese bezüglich der Lohnsatzentwicklung notwendig, da erst dadurch das System geschlossen wird. Hier geht man von einer Unabhängigkeit der nominellen Lohnsätze von der Entwicklung des Preisniveaus aus, wobei auch schon bestimmte lags der Anpassung die Verteilung determinierbar machen. So wird der Reallohn und damit die Verteilungssituation der Arbeitnehmer eine Variable in Abhängigkeit von der Entwicklung des Preisniveaus, welches wiederum vom Umfang der autonomen Ausgaben bestimmt wird. 


\section{b) Berücksichtigung von Verhaltensfunktionen}

Der entscheidende Schritt von der Keynesschen Verteilungsgleichung zu einem verteilungstheoretischen Modell besteht in der Einbeziehung einiger, wenn auch recht einfacher Verhaltensfunktionen: So werden die Sparfunktionen zweier Im Kreislaufprozeß an unterschiedlicher Stelle stehender Gruppen unterschieden, ferner wird eine Hypothese bezülich des Investitionsverhaltens der Unternehmer eingeführt. Die zunächst getroffene Annahme einer exogen gegebenen Investitionsquote wird noch bei Untersuchung des zu erwartenden Crowdingout kritisch überprüft werden müssen.

Kaldor geht von jeweils linear-homogenen Sparfunktionen für die Gruppe der Unternehmer und der Nicht-Unternehmer[83] aus, wobei die Sparneigung der Unternehmer immer über der der Arbeitnehmer liegt. Als Begründung für die Divergenz der beiden Sparneigungen wird von Kaldor auf den erheblichen Anteil der einbehaltenen Gewinne an der Ersparnisbildung der Unternehmer verwiesen[84]. Ging Kaldor noch von einer funktionellen Verteilungstheorie aus, bei der unterstellt wurde, daß die beiden Gruppen nur je eine spezifische Einkommensart beziehen - die Nichtunternehmer kontraktbestimmtes Einkommen, die Unternehmer Einkünfte aus Vermögen und Gewinne im weiteren Sinne wird nach Abschluß der Pasinetti-Diskussion[85] das Kaldor-Modell als Erklärungsansatz für die institutionelle Verteilung angesehen[86].

Eine Erklärung der Unterschiede in der Sparneigung über die Sparfähigkeit macht auch die Annahme Pasinettis plausibel, daß bei Bezug verschiedener Einkommensarten die gesamte durchschnittliche Sparneigung nicht zunehme, da hier Substitutionen der Ersparnisbildung aus verschiedenen Einkommens arten stattfinden. Von entsprechenden Strukturverschiebungen in der Querverteilung könnten zwar Rückwirkungen auf die Verhaltensparameter ausgehen, doch dürfte dies bei unserem Untersuchungsobjekt und der kurzfristigen Analyse, die Änderungen der Vermögensverteilung nicht endogen erfassen will, zu vernachlässigen sein.

\section{c) Darstellung des Modells}

Neben die schon oben eingeführten Gleichungen der Einkommensentstehung, der Einkommensverteilung, der Budgetgleichung und der Gleichgewichtsbedingung treten die homogen-linearen Sparfunktionen für die beiden verteilungsrelevanten Gruppen. Ausgangspunkt des Modells sind also folgende Gleichungen, die dem Modellcharakter entsprechend in Realgrößen definiert sind[87]:

$$
\begin{aligned}
& \text { III.1. } \quad \mathrm{Y}=\mathrm{C}+\mathrm{I}_{\mathrm{pr}}+\mathrm{A}_{\mathrm{st}} \\
& \text { III.2. } \quad \mathrm{Y}=\mathrm{Y}_{\mathrm{u}}+\mathrm{Y}_{\mathrm{nu}}
\end{aligned}
$$


III. 9. $\mathrm{A}_{\mathrm{st}}=\left(1-\mathrm{s}_{\mathrm{st}}\right) \mathrm{K}_{\mathrm{st}}$

III. 10. $S_{u}=s_{u} Y_{u}$

III. 11. $s_{n u}=s_{n u} Y_{n u}$

III. 12. $S=S_{u}+S_{n u}$

III. 13. $\mathrm{S}=\mathrm{I}+\mathrm{A}_{\mathrm{st}}$

Mit der Berücksichtigung der Nichtverausgabung in III.9. kann auch der Fall der Stillegung erfaßt werden. Diese geht nicht in die Gleichgewichtsbedingung III.13. dieses Realmodells ein, da sie keine reale Ersparnis darstellt. Da die beiden Verhaltensparameter $s_{u}$ und $s_{n u}$, die exogen determinierten Aggregate Ipr und $A_{S t}$ (sowie $s_{s t}$ ) und mit der Vollbeschäftigungsannahme auch das reale Sozialprodukt gegeben sind, lassen sich die Quoten der Primärverteilung aus obigem System ableiten.

Durch Substitution und Auflösung nach der Gewinnquote ergibt sich für $\mathrm{Y}_{\mathrm{u}} / \mathrm{Y}$ :

III. 14. $\frac{Y_{u}}{Y}=\frac{\frac{I_{p r}}{Y}+\left(1-s_{s t}\right) \frac{K_{s t}}{Y}-s_{n u}}{s_{u}-s_{n u}}$ oder

III. 15. $\frac{\mathrm{Y}_{\mathrm{u}}}{\mathrm{Y}}=\frac{\frac{\mathrm{I}_{\mathrm{pr}}}{\mathrm{Y}} \mathrm{s}_{\mathrm{nu}}}{\mathrm{s}_{\mathrm{u}}-\mathrm{s}_{\mathrm{nu}}}+\frac{\left(1-\mathrm{s}_{\mathrm{st}}\right) \frac{\mathrm{K}_{\mathrm{st}}}{\mathrm{Y}}}{\mathrm{s}_{\mathrm{u}}-\mathrm{s}_{\mathrm{nu}}}$

Bezüglich der Funktionsfähigkeit des Modells sind gewisse Grenzen für die Parameter zu beachten, die anhand Gleichung III.14. dargestellt seien. $\mathrm{Zu}-$ nächst muß die Bedingung $s_{u} \neq s_{n u}$ erfüllt sein, da andernfalls die Gleichung nicht determiniert wäre. Zudem wurde oben die Annahme begründet, daß $s_{u}>s_{n u}$ sei, was nach Kaldor die eigentliche Stabilitätsbedingung darstellt, da sich sonst bei Anpassungsprozessen kein Gleichgewicht mehr einstellen würde[88]. Aus diesen beiden Bedingungen folgt ferner, daß sich der Anteil der nicht konsumtiv verwendeten Güter am Sozialprodukt zwischen den beiden Sparneigungen bewegen muß, da diese mit den Einkommen gewichtet die gesamtwirtschaftliche Ersparnis ausmachen, so daß sich die Stabilitätsbedingung in Form folgender Ungleichung darstellen läßt:

III. 16.

$$
\left.s_{u}>\frac{I_{p r}+\left(1-s_{s t}\right) K_{s t}}{Y}\right\rangle s_{n u}
$$


Darüber hinaus gilt es noch rein qualitative Grenzen zu beachten, bei deren Überschreiten das System insgesamt gefährdet wäre. Diese Grenzen sind durch die Verteilungssituationen markiert, die das Spektrum möglicher Gleichgewichtssituationen begrenzen. So kann die Gewinnquote nicht beliebig zunehmen, da es für die reale Lohnquote eine - wie auch immer definierte - Untergrenze gibt. Andererseits darf die Gewinnquote nicht unter einen Wert fallen, der eine noch angemessene Verzinsung ermöglicht, da andernfalls die Annahmen der Exogenität der Investitionen und der Vollbeschäftigung nicht mehr gewährleistet wären[89].

Dem Charakter dieses Modells entsprechend ist auch die staatliche Kreditquote als Realgröße aufzufassen. Dies bedeutet, daß die nominale Größe staatlicher Schuldaufnahme zunächst unbestimmt ist, da mit Preissteigerungen im Zuge der Verausgabung zu rechnen ist, die auch auf das staatliche Ausgabenprojekt rückwirken werden. Da sich das staatliche Ausgabeverhalten aber durchaus an realen Güterkörben orientiert[90], ist diese real definierte Kreditnachfrage zumindest ebenso plausibel wie die in der klassischen Schuldtheorie uibliche Unterstellung der Zinsunempfindlichkeit staatlicher Kreditnachfrage. Problematischer ist dagegen die Bezugnahme auf das Sozialprodukt, d.h. die Quotenbetrachtung. Bei erstmaliger staatlicher Schuldaufnahme stellt dies kein Problem dar, da sich die Quote bei gegebenem realem Sozialprodukt rein definitorisch ergibl. Inwieweit eine solche Quote dann aber in der Folgeperiode Zielgröße des staatlichen Ausgabenverhaltens sein kann, wird in Kapitel IV. noch zu erörtern sein.

\section{d) Verteilungswirkungen}

Wie aus Gleichung III.14. ersichtlich kommt der staatlichen Schuldaufnahme im Kaldor-Modell bezüglich der Verteilungseffekte eine Rolle zu, die der der privaten Investitionen vergleichbar ist, sofern keine kompensierenden Bewegungen innerhalb des Aggregats der privaten Vermögensbildung zu erwarten sind. Zunächst sei aber der Kaldorsche Ablauf unterstellt, der als bisher noch nicht explizit angeführte, notwendige Voraussetzung die eines hinreichend elastischen Kreditsystems bedingt. Dies bedeutet, daß die monetären Voraussetzungen zur Finanzierung der in diesem Modell unvermeidlichen Preissteigerungen gegeben sein müssen, wenn die privaten Investitionen nicht tangiert werden sollen.

Der Einfluß der staatlichen Schuldaufnahme kommt unter dieser Annahme im additiven Glied von III.15. zum Ausdruck. Danach kann die staatliche Schuldaufnahme nur positive Impulse auf das Unternehmereinkommen ausüben. Lediglich für den Fall, daß die aufgenommenen Mittel in vollem Umfange (Stillegungskoeffizient $s_{s t}=1$ ) stillgelegt würden, ergäbe sich kein Verteilungs effekt zugunsten der Unternehmereinkommen. Diese Einbezlehung der Stillegung[91] soll nochmals daran erinnern, wie wesentlich die Verausgabung der Mittel und die damit verbundenen gütermäßigen Ansprüche an das Sozialpro- 
dukt in kreislauftheoretischer Sicht für die Verteilungswirkungen sind. In Höhe der nachfragewirksamen Verschuldung ergibt sich ein multiplikativer Verteilungseffekt zugunsten der Unternehmereinkommen, dessen Umfang von der Differenz der beiden Gruppensparneigungen bestimmt wird. Kaldor bezeichnet diese Größe als Sensitivitätskoeffizient, der für die Stabilität des Systems verantwortlich ist[92]. Da es sich um den Kehrwert der Differenz zweier echter Brüche handelt, wird dieser Multiplikator um so größer sein, je geringer die Differenz der beiden Sparquoten ist. Die Bedeutung für die Stabilität (des Systems) erklärt sich aus dem Wirkungsmechanismus des Modells, der bei Gleichgewichtsstörungen durch erhöhte Staatsverschuldung solange Einkommensumschichtungen zugunsten der Gruppe mit der höheren Sparneigung erforderlich macht, bis die gesamtwirtschaftlich erforderliche Ersparnisbildung erreicht ist. Je geringer nun diese Differenz, desto größer müssen bei gleichen Absolutbeträgen die Verteilungsänderungen sein.

Das hier vorliegende Ergebnis unterscheidet sich vom einfachen Fall der Keynessschen Verteilungsgleichung, bei der sich Verteilungsänderungen nur im Umfange der staatlichen Schuldaufnahme ergaben, durch die multiplikativen Effekte. Im folgenden soll eine Erklärung für diese Divergenzen gegeben werden, die gleichzeitig die jeweils unterstellten Wirkungsabläufe kritisch hinterfragt.

e) Vergleich der Ergebnisse nach Kaldor und der Keynesschen Verteilungsgleichung

Die Unterschiede zwischen der Aussage der einfachen Keynesschen Verteilungsgleichung und dem Modell Kaldors liegen allein im quantitativen Effekt, den eine Kreditfinanzierung von Staatsausgaben zugunsten der Unternehmereinkommen erzeugt. Zeigt die einfache Verteilungsgleichung ein erhöhtes Unternehmereinkommen im Umfange der Staatsverschuldung an, wobei die Wirkungsabläufe ohne Erklärungshypothese bleiben, so ergibt sich beim differenzierteren Kaldor-Ansatz ein multiplikativer Effekt, dessen Ausmaß von den Verhaltensparametern bestimmt wird. In der Keynesschen Verteilungsgleichung wird lediglich die Bedingung umschrieben, daß die zusätzliche Vermögensbildung von den Unternehmern geleistet wird. Das Kaldor-Modell umfaßt dagegen zusätzliche Verhaltensannahmen, die die "Umverteilung" im Rahmen eines Multiplikatorprozesses erklären.

Ginge man hypothetisch davon aus, daß die Zusatzeinkommen der Unternehmer in Gestalt der Kreislaufgewinne voll gespart würden[93], die marginale Sparneigung also bei eins läge, so ergäbe sich unabhängig von der Sparneigung der Arbeitnehmer der in der Keynesschen Verteilungsgleichung geschilderte Fall. Damit wird der Unterschied im Ablauf der beiden Ansätze deutlich, denn in der Keynesschen Verteilungsgleichung wird der Konsum der Unternehmer als Absolutgröße konstant gehalten, während er bei Kaldor gemäß der Konsumneigung als relativ konstant betrachtet wird. 
Die Ausrichtung der Spar-bzw. Konsumneigung auf reale Größen ist eine nicht unproblematische Annahme[94]. Dies gilt insbesondere für die unterstellte Konstanz der realen Sparneigung der Arbeitnehmer, derzufolge bei steigenden Preisen und konstantem Nominaleinkommen die nominale Sparsumme konstant blelben muß. Für das Unternehmerverhalten ist diese Unterstellung, daß keine Geldillusion vorliegt, unproblematisch, da eine Erhöhung der nominalen Sparbeträge bei auch absolut zunehmendem Nominaleinkommen nicht unplausibel erscheint. Wir haben oben schon darauf verwiesen, daß bei im Kreislauf anfallenden windfall-profits auch eine marginale Sparneigung von eins nicht überraschen würde[95], mutmaßliche Veränderungen des Sparverhaltens der Unternehmer würden aber in eine solche Richtung verlaufen, die auf den Umfang der Verteilungseffekte dämpfend wirken würden. Zieht man diese Überlegungen mit heran, so werden sich die tatsächlich ergebenden Verteilungseffekte zwischen den beiden Extremen bewegen. In jedem Fall ist unter den modellimmanenten Annahmen eine Verbesserung der Verteilungsposition der Unternehmer zu erwarten, die sogar ein Mehrfaches des ursprünglichen Impulses ausmachen kann. Analogien zur mehr als vollständigen Überwälzung bei anderen staatlichen Einnahmeinstrumenten liegen nahe.

\section{f) Variation der Sparneigungen}

Die bisherigen Überlegungen wurden unter Annahme der Konstanz der Verhaltensparameter getroffen, lediglich mögliche Rückwirkungen von Kreislaufgewinnen auf diese Größen wurden angesprochen. Dabei wurde aufgezeigt, daß eine Erhöhung der Sparneigung aufgrund von Kreislaufgewinnen die notwendige Umverteilung zugunsten der Unternehmer reduziert. Aber auch eine Veränderung der Sparleistung der Arbeitnehmer beeinflußt die aufgrund einer staatlichen Verschuldung eintretenden Verteilungseffekte.

Im folgenden sollen deshalb die Verteilungswirkungen einer Staatsverschuldung untersucht werden, die aus zusätzlicher Ersparnis der Arbeitnehmer alimentiert wird. Mit dieser Annahme soll keine Aussage über die Wahrscheinlichkeit einer solchen Konstellation verbunden sein. Da sich eine solche Verhaltensänderung auf die zusätzliche Staatsverschuldung hin nicht unmittelbar in der relativen Größe der Sparneigung erfassen läßt, sei von der Gleichgewichtsbedingung des Gleichungssystems ausgegangen. Es sei unterstellt, daß im Umfange der zusätzlichen nachfragewirksamen Kreditaufnahme $\mathrm{K}_{\mathrm{St}}$ auch vermehrt von den Nicht-Unternehmern gespart wird. Die zusätzliche Sparquote bemißt sich dann am Anteil von $\mathrm{K}_{\mathrm{st}}$ am Gesamteinkommen dieser Gruppe, d.h. $\Delta \mathrm{s}_{\mathrm{nu}}=$ $\mathrm{K}_{\mathrm{st}} / \mathrm{Y}_{\mathrm{nu}}$. Unsere Gleichgewichtsbedingung lautet nun $\mathrm{V}_{\mathrm{pr}}=\mathrm{I}_{\mathrm{pr}}+\mathrm{K}_{\mathrm{st}}=$ $s_{u} \cdot Y_{u}+s_{n u} \cdot\left(Y-Y_{n u}\right)+\Delta s_{n u} \cdot\left(Y-Y_{n u}\right)$. Setzt man für den letzten Summanden die obige Bestimmungsgleichung ein, so verschwindet die staatliche Kreditnahme und ihr Gegenpart, die Ersparnisbildung, aus dem System, was nichts anderes bedeutet, als daß sich ein unverändertes Verteilungsbild ergibt. 
Entsprechende Überlegungen lassen sich für eine Mehrersparnis der Unternehmer oder aber beider Gruppen treffen. In jedem Falle einer zusätzlichen Ersparnisbildung in Höhe der staatlichen Kreditnahme ergibt sich dann, daß keine Auswirkungen auf die Primärverteilung auftreten.

Die Beurteilung der Verteilungswirkungen der Staatsverschuldung bei gleichzeltig ansteigender Ersparnisbildung der Arbeitnehmer muß zunächst zwiespältig bleiben. Zwar treten die oben aufgezeigten negativen Verteilungseffekte nicht auf, die ohne diese Parameteränderung unvermeidlich wären, doch wäre eine solche Anregung zur Ersparnisbildung ohne begleitende Kreditauf nahme des Staates verteilungspolitisch wirkungsvoller. Entsprechend den kreislauftheoretischen Aussagen hätte dies isoliert betrachtet zu einer Verbesserung der Verteilungsposition der Nicht-Unternehmer führen müssen. Dann wäre nämlich der in der vermögenspolitischen Diskussion vielfach zitierte Fall der Vermögensbildung ohne Konsumverzicht realisiert, während im Falle der begleitenden Staatsverschuldung eine Vermögensbildung mit Konsumverzicht zugunsten des Staates stattfindet. Für den Fall einer erhöhten Ersparnis der Unternehmer wäre für die Gruppe der Arbeitnehmer weder Konsumverzicht noch Vermögensbildung zu konstatieren[96].

Geht aber eine solche Verhaltensänderung kausal auf die staatliche Schuldaufnahme zurück, so ist gegen diese saldierende Betrachtung nichts einzuwenden, entspricht sie doch dem hier zugrundegelegten Inzidenzkonzept.

Allerdings ist der Unterstellung einer solchen Kausalität große Skepsis entgegenzubringen, da selbst besonders attraktive Konditionen zunächst zu einer Umschichtung der Vermögensanlagen, nicht aber zu einer Vermögensneubildung führen werden[97]. So darf aus einer bestimmten Anlageform keinesfalls auf eine Zunahme der absoluten Ersparnis geschlossen werden. Erst nach Analyse der gesamtwirtschaftlichen Zusammenhänge der Vermögensbildung kann beurteilt werden, ob tatsächlich eine zusätzliche Ersparnisbildung erfolgte und damit keine negativen Effekte auf die Einkommensverteilung zu erwarten sind.

\section{g) Berücksichtigung eines Crowding-out}

Die bisherigen Überlegungen zu den Verteilungswirkungen der staatlichen Schuldaufnahme gingen davon aus, daß in vollem Umfange der zusätzlichen, staatlichen Ansprüche die private Vermögensbildung zunehmen würde[98]. Schon bei der Keynesschen Verteilungsgleichung wiesen wir aber auf die $\mathrm{Be}-$ deutung eines möglichen Crowding-out für die Entwicklung der Einkommensverteilung hin, auch im Modell Kaldors gelten diese Zusammenhänge. Im klassischen Extremfall der völligen Verdrängung der privaten Investitionen ergäbe sich eine unveränderte Einkommensverteilung bei (nahezu) konstanter Vermögensbildung. Im privaten Sektor geht zwar die Realinvestition zurück, die Geldvermögensbildung nimmt dagegen aufgrund des Außenverhältnisses des Staates $z u$, es ergäbe sich lediglich eine Umstrukturierung der Ver- 
mögensarten. Da dieser Extremsituation aber wohl nur Sonderfall-Charakter zukommt, muß man dahingehend relativieren, daß in dem Umfange, in dem die private Investitionsgüternachfrage zurückgeht, von der staatlichen Nachfrage keine Veränderung der Primärverteilung der betrachteten Periode zu erwarten ist[99]. Angesichts der Bedeutung der Crowding-out-Effekte für die Verteilung müssen die Bestimmungsfaktoren der Beziehungen im Vermögensaggregat näher untersucht werden, was zunächst zu einer Betrachtung der Determinanten des privaten Investitionsverhaltens führt. Da Kaldor von einer exogen gegebenen Investitionsquote ausgeht, kann dieses Untersuchungsziel auch so formuliert werden, daß die Plausibilität dieser Annahme bei einer zusätzlichen staatlichen Kreditnahme zu überprüfen ist.

\section{Determinanten der Investitionsnachfrage}

a) Verhältnis von Investitionsneigung $\mathrm{zu}$ Investitionsmöglichkeiten

Bei den bisherigen Überlegungen zu den Verteilungseffekten staatlicher Verschuldung wurde nur hypothetisch ein Crowding-out angenommen, ansonsten gingen wir aber in modellimmanenter Argumentation von Kaldors Wirkungs mechanismen aus, nach denen ein hinreichend flexibles Kreditsystem unterstellt wird, um alle autonomen Ansprüche finanzieren zu können. Damit ist in Gestalt der Investitionsmöglichkeiten eine notwendige, wenn auch nicht hinreichende Bedingung zur Aufrechterhaltung der privaten Investitionsnachfrage genannt[100]. Ob diese Voraussetzung als gegeben angenommen werden kann und inwieweit dies von Verschuldungsort und -art abhängig ist, soll im folgenden Abschnitt untersucht werden. Die bisherige Annahme hinreichender Elastizität des Bankensystems bedeutete ein ebenso apodiktisches Vorgehen wie die Annahmen der klassischen Schuldtheorie bezüglich der kompensierenden, geldpolitischen Eingriffe.

Hinreichende Bedingung für die Aufrechterhaltung der Investitionsgüternachfrage ist dann, daß die gegebenen Investitionsmöglichkeiten auch beansprucht werden. Um diese Frage beantworten zu können, müssen die ins Investitionskalkül der Unternehmer einfließenden Faktoren auf ihre Bedeutung hin untersucht werden, denn trotz vorhandener Investitionsmöglichkeiten könnte die Investitionsneigung zurückgehen. Der Charakter der notwendigen Voraussetzung der Investitionsmöglichkeit kommt dagegen zum Ausdruck, wenn bei zwar vorhandener Investitionsneigung, aber nicht gegebener Investitionsmöglichkeit die Investitionsnachfrage zurückgehen muß. 
b) Auswirkungen einer staatlichen Schuldaufnahme auf die private Investitionsnachfrage

Für die klassische Schuldtheorie sind die in Verbindung mit einer zusätzlichen staatlichen Kreditnachfrage zu erwartenden Zinssteigerungen die entscheidende Größe, da ausschließlich diese die Verdrängung der privaten Investitionen bewirken. Der Zinssatz ist in der klassischen Theorie zum einen Indikator der Investitionsmöglichkeiten, zum anderen geht er als entscheidende Größe in das Investitionskalkül ein[101]. Diese monokausale Sicht der Investitionsdeterminanten ist aber nicht aufrecht zu erhalten. Kommt dem Zinssatz auch noch Indikatorfunktion für die Investitionsmöglichkeiten $z u$, so spielt diese Größe für die Investitionsneigung eine nur marginale Rolle.

Die Investitionsneigung, d.h. das Ausmaß der gewünschten Investitionstätigkeit, bestimmt sich aus der Grenzprofitrate oder Grenzleistungsfähigkeit des Kapitals und den subjektiv bestimmten Mindestrenditen. Diese Größe der gewünschten Rendite ist vom Marktzins weitgehend unabhängig, aber auch für die Rentabilitätsermittlung spielt der Zins nur unter bestimmten Voraussetzungen eine Rolle[102]. Handelt es sich nämlich um im Preiserschen Sinne Unternehmerkapitalisten, so ist für diese der gesamte Kapitalgewinn Orientierungsgröße, der Marktzins ist dann ex definitione nicht von Bedeutung. Anders dagegen in dem Falle, wenn der Unternehmergewinn im engeren Sinne Entscheidungskriterium für ein Investitionsvorhaben ist. Hier ist der Marktzins (und seine Veränderungen) von Bedeutung, wenn er als Kostenfaktor ins Gewicht fällt. Davon ist aber ein relativ geringer Anteil an den gesamtwirtschaftlichen Investitionen betroffen[103], so daß der Einfluß des Zinssatzes auf die Investitionsneigung nahezu vernachlässigt werden kann.

Anstelle der Zinssatzveränderungen gilt es, die Auswirkungen einer staatlichen Schuldaufnahme auf die Gewinn- und Absatzerwartungen zu berücksichtigen, die für das Ausmaß der gewünschten Investition entscheidend sind. Sind damit die Auswirkungen auf die Investitionsneigung erfaßt, so muß anschließend gefragt werden, ob auch die hinreichende Bedingung in Gestalt der Investitionsmöglichkeiten gegeben, die gewünschte Investition also auch finanzierbar ist.

Aufgrund einer ausgabenwirksamen, staatlichen Kreditaufnahme ergibt sich (unter dem Vorbehalt gegebener Investitionsmöglichkeiten) eine Nachfrageexpansion, die sich tendenziell in einer Erhöhung der Kreislaufgewinne niederschlägt. Die Gewinnsituation der Unternehmen wird sich also verbessern. Unter Berücksichtigung dieser Faktoren, die als eindeutig dominierende Argumente in der gesamtwirtschaftlichen Investitionsfunktion anzusprechen sind, ist ein Crowding-out aufgrund eines Rückgangs der Investitionsneigung völlig ausgeschlossen, wirken die von der staatlichen Kreditaufnahme ausgehenden Impulse doch insgesamt stimulierend auf die Investitionsneigung.

Damit werden die Investitionsmöglichkeiten zum entscheidenden Faktor für ein mögliches Crowding-out. Lediglich für den Fall, daß die Mittel zur Finanzierung aller Nachfragewünsche nicht zur Verfügung stehen, könnte das von der 
klassischen Theorie angenommene Crowding-out tatsächlich eintreten, nur von Zinssatzsteigerungen sind aber keine restriktiven Effekte zu erwarten[104]. Damit ist der klassische Wirkungsmechanismus gewissermaßen auf den Kopf gestellt, ziehen nun doch staatliche Ansprüche private nach sich.

Im folgenden soll nun den Bestimmungsfaktoren nachgegangen werden, die das quantitative Ausmaß des Angebots an Finanzlerungsmitteln, also die Elastizität des Geld- und Kreditangebots, bestimmen.

\section{Elastizität des Geld-und Kreditangebots}

a) Rolle des Bankensystems

Die Investitionsmöglichkeiten werden so zur Schlüsselgröße für die interpersonellen Verteilungseffekte[105], da von seiten der Investitionsneigung keine kontraktiven Impulse auf das Investitionsverhalten zu erwarten sind, im Gegenteil sogar eine Erhöhung der Investitionsneigung anzunehmen ist.

Indem man den Investitionsmöglichkeiten eine solch entscheidende Rolle zuerkennt, werden die Geldschöpfungs möglichkeiten der Banken für die Verteilungseffekte wesentlich. Das Bankensystem nimmt eine Art Pufferfunktion gegenüber exogenen Einflüssen auf das System ein, indem es zu endogenen Variationen des Geldangebots in der Lage ist[106]. Eine gewisse, so verstandene Endogenität[107] ist aber konstitutives Merkmal entwickelter Volkswirtschaften mit entsprechendem Geldsystem. Damit entfernt man sich von den starren availablefunds-Vorstellungen klassischer Prägung, denenzufolge das Konkurrieren um ein vorgegebenes Kreditangebot bei entsprechend geringer Zinsempfindlichkeit des Staates zwangsläufig zu einem Rückgang der Investitionsmöglichkeiten für die Privaten führen muß.

An dieser Stelle soll schon einschränkend bemerkt werden, daß es sich bei der Frage der Elastizität oder des Liquiditätsstatus des Bankensystems nicht nur um eln qualitatives, sondern auch (und vor allem) um ein quantitatives Problem handelt, da die Endogenität des Geld- und Kreditangebots nur im Verhältnis zu der zusätzlichen Geldnachfrage beurteilt werden kann.

Um zu annähernd die Realität erfassenden Aussagen zu gelangen, die ïber den rein konditionalen Charakter der deus-ex-machina-Vorstellungen von der Geldpolitik der klassischen Schuldtheorie hinausgehen[108], sollen im folgenden die Rückwirkungen unterschiedlicher Formen der Staatsverschuldung auf die Liquiditätssituation des Bankensystems untersucht werden. Da hier mögliche kontraktive Effekte im Vordergrund stehen, wird auf eine Zentralbankverschuldung nicht eingegangen[109]. Deren Wirkungen finden in der Literatur eine einmütige Beurtellung. So wird nach lang- und kurzfristiger Verschuldung beim Geschäftsbankensystem differenzlert, mögliche Unterschiede, die sich durch eine unmittelbare Verschuldung bel den privaten Nicht-Banken ergeben, sollen am Rande vermerkt werden[110] [111]. 
Zur Beurteilung der Wirkungen auf die Liquiditätssituation des Bankensystems ist es angebracht, zunächst auf deren Bestimmungsfaktoren einzugehen. Damit löst man sich von der rein abstrakten Betrachtungsweise, da diese Zusammenhänge entscheidend von institutionellen Gegebenheiten geprägt sind. Wir werden deshalb den institutionellen Rahmen und die damit gemachten Erfahrungen in der Bundesrepublik in die Betrachtung einbeziehen. Dabei soll dem Inzidenzkonzept entsprechend von kompensierenden geldpolitischen Maßnahmen abstrahiert werden. Unsere Überlegungen werden es dann aber ermöglichen, eine Beurteilung der geldpolitischen Eingriffs möglichkeiten vorzunehmen, die von der klassischen Schuldtheorie als gegeben unterstellt werden.

\section{b) Bestimmungsfaktoren der Elastizität des Bankensystems}

Prinzipielle Voraussetzung zur Giralgeldschöpfung des Bankensystems, und in dieser Fähigkeit drückt sich die Endogenität des Geldangebots aus, ist die Verfügbarkeit über Zentralbankgeld. Da von Reaktionen der Zentralbank zunächst abgesehen wird, muß sich also Zentralbankgeld in Händen der Geschäftsbanken befinden bzw. diese müssen sich solches beschaffen können, um den zunehmenden Bedarf zu befriedigen[112]. Geht man davon aus, daß die Banken aufgrund der Unverzinslichkeit nur geringe Bestände an nicht gebundenem Zentralbankgeld (Überschußreserven) halten[113], so rücken die frei verfügbaren Liquiditätsreserven bzw. die Sekundärliquidität in den Blickpunkt, da sie Indikator für die Möglichkeiten des Bankensystems sind, sich zusätzliche Primärliquidität in Form von Zentralbankgeld zu beschaffen. Neben den schon erwähnten Überschußreserven sind zunächst die Refinanzierungs möglichkeiten bei der Zentralbank in Form unausgenutzter Diskont- und Lombardkontingente zu nennen, daneben aber auch die inländischen staatlichen Geldmarktpapiere, sofern sie jederzeit an die Zentralbank zurückgegeben werden können[114]. Den zumindest früher bei weitem wichtigsten Faktor, der allerdings von der Ausgestaltung des Wechselkurssystems abhängt, stellt die außenwirtschaftliche Komponente der Zentralbankgeldschaffung dar. Bei festen Wechselkursen ist die Bundesbank verpflichtet, beliebige Mengen an Devisen anzukaufen[115], so daß diese Rückgriffsmöglichkeiten eine nahezu völlige Endogenität des inländischen Geldangebots mit sich bringen. So war auch die monetäre Situation in der Bundesrepublik lange durch eine entsprechend hohe Elastizität des Geldangebots gekennzeichnet[116]. Der Übergang zu einem System flexibler Wechselkurse eliminiert im Idealfall diese Möglichkeit der Geschäftsbanken[117], da ohne Eingreifen der Zentralbank nun jede Devisentransaktion im Sinne eines echten Tausches ohne Mitwirkung der Zentralbank abläuft, die inländische Zentralbankgeldmenge also von diesen Einflüssen völlig unberührt bleibt[118]. 
Im folgenden sei der Vereinfachung halber davon ausgegangen, daß der Staat über Konten bei den Geschäftsbanken verfügt. Dann räumen die Banken dem Staat bei Übernahme von Staatspapieren Sichtguthaben ein, wofür sie entsprechend den Mindestreserveverpflichtungen Zentralbankgeld aufbringen müssen[119]. Halten die Banken Überschußreserven, d.h. über die Mindestreserven hinausgehende Zentralbankgeldmengen, oder ist ihnen die Umwandlung von Sekundärliquidität in Primärliquidität möglich, so stellt die Befriedigung der zusätzlichen staatlichen Kreditnachfrage kein Problem dar; möglicherweise auftretende Zinseffekte sollen hier nicht weiter verfolgt werden[120]. Aus diesen allgemeinen Überlegungen wird deutlich, daß lediglich die Nicht-Verfügbarkeit über (aktuelles oder potentielles) Zentralbankgeld eine durch die staatliche Nachfrage notwendig werdende Ausdehnung der Investitionsmöglichkeiten verhindern könnte[121].

Für diesen Fall müßten Hypothesen bezüglich des Bankenverhaltens getroffen werden, da nicht a priori unterstellt werden kann, daß diese einer - auch wenn als zinsrobust unterstellten - staatlichen Kreditnachfrage nachkommen. In diesem Zusammenhang ist an eine besondere Eigenschaft bestimmter kurzfristiger Staatspapiere zu denken, die deren Attraktivität für die Geschäftsbanken erklärt. In die Geldmarktregulierung einbezogene kurzfristige Papiere können nämlich jederzeit an die Zentralbank zurückgegeben werden und stellen somit einen bedeutenden Posten der Sekundärliquidität des Bankensystems dar. Allerdings ist diese Eigenschaft an eine entsprechende Zusage der Bundesbank gebunden. Gerade in jüngster Zeit läßt sich beobachten, daß die kurzfristigen Staatspapiere nicht, mehr in die Geldmarktregulierung einbezogen sind. "Zentralbankgeld und kurzfristige Staatsschuldtitel können nämlich zumindest für die Wirtschaftseinheiten des finanziellen Sektors sehr enge, wenn nicht sogar vollständige Substitute darstellen. Mit anderen Worten: die Geschäftsbanken und andere finanzielle Institute betrachten Zentralbankgeld und kurzfristige staatliche Wertpapiere liquiditäts mäßig als nahezu gleichwertig"'[122]. Wird eine solche Rücknahmeverpflichtung eingeräumt, hat die Zentralbank über diese Bestände an potentieller Primärliquidität keine Gewalt.

Geht man zunächst einmal davon aus, daß im Umfang des staatlichen Kredits andere Kreditzusagen abgeblockt werden, so würde bereits eine teilweise Rückgabe der Papiere genügen, auch die privaten Kreditwünsche befriedigen zu können. Die Banken hätten darüber hinaus sogar die Möglichkeit, zusätzliche Kredite zu vergeben. An dieser Stelle muß allerdings auf den hypothetischen Charakter dieser Argumentation in Extremen verwiesen werden, da in einer solchen Situation wohl kaum zu diesem Finanzierungsinstrument gegriffen würde.

Handelt es sich dagegen um eine langfristige Verschuldung, so kann ein Crowding-out dann eintreten, wenn die Geschäftsbanken ihr Aktivgeschäft mit privaten Wirtschaftssubjekten zugunsten des Staates einschränken. Lediglich in diesem Falle ergibt sich ein Wirkungsmechanismus gemäß den klassischen Vorstellungen, wobel als Voraussetzungen keinerlei Bestände an Sekundärliquidität 
(was ein idealtypisches flexibles Wechselkurssystem impliziert) und ein Präferieren der Kreditgewährung an den Staat zu nennen sind. Bezieht man die Möglichkeit der Verschuldung bei privaten Nichtbanken ein, für die die Rückgriffsmöglichkeit auf Zentralbankgeld keine Rolle spielt, so ergeben sich nur für die letztgenannte Situation einer gegen Null gehenden Sekundärliquidität der Geschäftsbanken restriktive Effekte[123]. Diese Verschuldungsform ist also einer langfristigen Verschuldung im Bankensystem vergleichbar.

d) Erfolgs möglichkeiten kompensierender geldpolitischer Eingriffe

Obige Ausführungen sollten die zentrale Bedeuturg der Liquiditätssituation des gesamten Bankensystems deutlich gemacht haben, von deren Zustand die Verdrängungseffekte im klassischen Sinne determiniert werden. Wie gezeigt wurde, gilt diese Aussage unabhängig vom jeweiligen Verschuldungsort; im Falle der Zentralbankverschuldung wird dieses Problem uberhaupt nicht aktuell. Wie auch bei der Vermögensbildung müssen alle monetären Märkte betrachtet werden, da durch Substitutionsbeziehungen unterschiedliche Verschuldungsorte eng mit einander verbunden sind. Eine rein formale Betrachtungsweise, die lediglich auf den Erwerber der staatlichen Papiere abstellt, kann zu keinen gehaltvollen Ergebnissen führen[124].

Entscheidende Bedeutung kommt diesem Phänomen deshalb zu, weil die Liquiditätssituation u.U. durch geldpolitische Bemühungen nur wenig beeinflußbar ist. Dann aber wird die normative Prämisse der klassischen Inzidenztheorie, die Geldpolitik habe dafür zu sorgen, daß nur ein bestimmtes Kreditvolumen zur Verfügung stehe, um so Preiseffekte im Ansatz zu verhindern, inhaltsleer bzw. ir real. Ein unüberwindliches Hindernis kann dabei schon die Ausgestaltung des Wechselkurssystems sein. Liegt ein System fester Wechselkurse vor, so ist die inländische Liquidität kaum steuerbar, aber auch bei flexiblen Wechselkursen, die zumindest eine unkontrollierbare Beeinträchtigung von der außenwirtschaftlichen Flanke her verhindern werden[125], kann sich diese klassische Bedingung als unerfüllbar erweisen, wenn die Geschäftsbanken über entsprechende $\mathrm{Be}$ stände an Sekundärliquidität verfügen. "Die zu neutralisierenden Beträge könnten dann nämlich kurzfristig das Kompensationspotential der Bundesbank übersteigen. Die Bundesbank hat es jedoch bei der gegebenen Absicherung nach außen in der Hand, die freien Liquiditätsreserven der Kreditinstitute niedrig zu halten'"[126]. Auch wenn man so aufgrund der in den letzten Jahren geänderten institutionellen Bedingungen für die Bundesrepublik zum Schluß kommen kann, daß eine kompensierende Geldpolitik möglich scheint[127], ist damit noch keine Aussage über die sich tatsächlich einstellenden Wirkungen in einer konkreten Situation getroffen.

Oben wurde schon auf die Bedeutung der quantitativen Abstufung hingewiesen. Da ein bestimmter Umfang an Sekundärliquidität immer vorhanden sein wird, ist in diesem Rahmen auch eine Endogenität des Geldangebots anzunehmen[128]. 
Bei der Diskussion der Endogenität des Geld- und Kreditangebots wurde aus schließlich auf die Rolle der Banken abgestellt, für den privaten Sektor wurde damit implizlt von elner Konstanz der Verhaltensweisen ausgegangen. Löst man aber diese vereinfachende Annahme auf, so mulssen auch Veränderungen der Umlaufsgeschwindigkeit des Geldes in das Kalkül einbezogen werden, die in ihren Wirkungen Geldmengenexpansionen oder -kontraktionen vergleichbar sind. Hierin ist ein zusätzlicher Faktor für die Annahme einer gewissen Endogenität zu sehen.

Zudem stellt sich im Falle einer staatlichen Schuldaufnahme das Problem, ob die unterschiedlichen Entscheidungsinstanzen, nämlich Regierung und Notenbank, überhaupt eine solche Absprache bezüglich geldpolitischer Kompensation zu treffen vermögen[129]. Hier soll nur auf das Koordinationsproblem verwiesen werden, die konstitutiven Diagnose- und Prognoseprobleme seien vernachlässigt. Auch wenn bei einem System flexibler Wechselkurse die generelle Steuerbarkeit der Zentralbankgeldmenge durch eine entsprechend rigorose Geldpolitik möglich scheint, könnten mit einem solchen Vorgehen doch folgenschwere Nebenwirkungen verbunden sein. "Es wird daher [allerdings] von der Annahme ausgegangen, daß die geld- und kreditpolitischen Instrumente in solcher Dosierung zum Einsatz kommen, daß beispielsweise ihre Restriktionswirkung nicht um jeden Preis erzielt werden soll; ein solcher Preis könnte die Gefährdung des wirtschaftlichen Wachstums oder der Verzicht auf marktwirtschaftliche Grundsätze sein"[130]. Die Annahmen der klassischen Schuldtheorie bezüglich der Rolle der Geldpolitik sind dann äußerst kritisch zu beurteilen, wenn durch restriktives Eingreifen der Geldpolitik Stabilisierungskrisen heraufbeschworen werden[131]. Zudem drängt sich die Frage nach der Rationalität einer so konzipierten staatlichen Einnahmepolitik auf.

\section{Ergebnis}

Die kreislauftheoretische Untersuchung der Verteilungswirkungen einer staatlichen Schuldaufnahme auf die Primärverteilung derselben Periode hat zweierlei deutlich werden lassen. Als entscheidendes Ergebnis muß die in verteilungspolitischer Sicht ausgesprochen negative Beeinflussung der Einkommensverteilung hervorgehoben werden, die sich aus den Kreislaufzusammenhängen ergibt. Hierbei sind Verteilungsumschichtungen möglich, die ein Mehrfaches der staatlichen Schuldaufnahme ausmachen können. Auch die kreditfinanzierten staatlichen autonomen Ansprüche wirken also verteilungsdeterminierend. Sie sind nicht von der Einkommensverteilung und der damit verbundenen Ersparnis abhängig. Da auch der andere wesentliche Bestandteil der autonomen Nachfrage, die privaten Investitionen, durch die staatlichen Ansprüche nicht negativ tangiert wird, sondern zusätzliche positive Impulse erhält, könnte lediglich eine entsprechend restriktiv wirkende Geldpolitik diese verteilungspolitische Beurteilung modifizieren[132]. 
Wurde obiges Ergebnis unter der Prämisse erzielt, daß keine kompensierenden geldpolitischen Eingriffe erfolgen, so muß als zweites Resultat dieser Überlegungen festgehalten werden, daß solche Eingriffe auch nur unter ganz bestimmten Voraussetzungen als möglich erscheinen. Wenn aber die von der klassischen Theorie als selbstverständlich zugrundegelegten Crowding-outEffekte nicht einmal durch diskretionäre Eingriffe erzielbar sind, so gewinnen die hier beschriebenen Abläufe an zusätzlicher Erklärungskraft.

Damit kann keine Eindeutigkeit der Wirkungen unterstellt werden. Im Einzelfall sind bei einer Vollbeschäftigungssituation als Ausgangslage die oben beschriebenen Crowding-out-Effekte auch durch geldpolitische Eingriffe herbeiführbar, aber eben nur bei Erfüllung bestimmter institutioneller Voraussetzungen und weiterer einengender Annahmen als wahrscheinlich anzusehen.

Nimmt man einen solchen Fall trotz dieser Einschränkungen als gegeben an, so wird die Primärverteilung, die mit der institutionellen Verteilung gleichzusetzen ist, unverändert bleiben. Unter Annahme klassischer Wirkungsmechanismen ergibt sich dann in kreislauftheoretischer Betrachtung eine hinreichend plausible Begründung für die Vernachlässigung der Auswirkungen einer staatlichen Schuldaufnahme auf die Einkommensverteilung der Ausgangsperiode in der klassischen Schuldtheorie.

Allerdings kann diese Begründung für den klassischen Ansatz nicht befriedigen, da sie nicht modellimmanent erfolgt. Es sollen deshalb noch einige Überlegungen zu diesem Problemkreis innerhalb eines grenzproduktivitätstheoretischen Ansatzes angestellt werden, da dieser die neoklassischen Vorstellungen entsprechende Methodik darstellt. Damit ist zugleich die Zielsetzung verbunden, die verteilungspolitische Aussagefähigkeit einer grenzproduktivitätstheoretischen Betrachtung der staatlichen Kreditnahme zu prüfen.

Vor diesen Überlegungen im Rahmen eines gänzlich anderen Erklärungskalküls soll allerdings noch ein Exkurs eingeschoben werden, der sich im Rahmen des bisher verwendeten theoretischen Gerüsts mit den möglichen Aussagen zu den Verteilungswirkungen der Staatsverschuldung in einer Situation der Unterbeschäftigung befassen soll.

6. Exkurs: Zu den Verteilungswirkungen der Staatsverschuldung in einer Situation der Unterbeschäftigung

Die bisherigen Überlegungen zu den Verteilungseffekten einer zusätzlichen Staatsverschuldung befaßten sich mit einer Vollbeschäftigungssituation, für die charakteristisch war, daß die gütermäßigen Ansprüche des Staates zu Lasten einer der anderen Verteilungspole gehen mußten. Das Konkurrieren um die knappen Ressourcen ist konstitutiv für das oben als das strukturelle bezeichnete Verteilungsproblem. Entsprechend ergab sich für die Verteilungswirkungen infolge einer staatlichen Kreditnahme, daß diese vom jeweiligen Umfang des Crowding-out abhängig sind, wobei sich die Verteilungsänderungen bei ge- 
gebenem realem Output ausschließlich über Preisniveauveränderungen einstellten.

In einer Phase der Unterauslastung des gesamtwirtschaftlichen Produktions potentials sind diese Zusammenhänge nicht mehr in der gleichen Weise gegeben. Eine zusätzliche Güterbeanspruchung muß keineswegs die Verschlechterung der Verteilungssituation einer anderen Gruppe bedeuten, sondern kann sogar für alle Gruppen absolute Verbesserungen mit sich bringen, da die Anpassungen des Systems auf den exogenen Impuls einer kreditfinanzierten staatlichen Nachfrage (hin zu einem neuen Gleichgewichtszustand) nun auch oder sogar ausschließlich uiber Reaktionen des Outputs laufen. Aus dem Umstand, daß Mengenänderungen für die Verteilungserklärung wesentlich werden, ist ersichtlich, daß das Verteilungsmodell Kaldors allein diese Abläufe nicht mehr zu erklären vermag[133]. Dennoch sollen einige nachfragetheoretisch orientierte Verteilungsüberlegungen angestellt werden, um zumindest die verteilungsdeterminierenden Faktoren und gleichzeitig die Bedeutung des verteilungspolitischen Problems in dieser Situation aufzuzeigen.

Erhöht der Staat in einer solchen konjunkturellen Ausgangssituation seine autonomen Ansprüche an das Sozialprodukt, so bedeutet dies zunächst eine Erhöhung der nominellen Gesamtnachfrage[134], die im Kreislauf zu den Unternehmern fließt. Gleichzeitig wird diese Nachfrageerhöhung aber auch eine Ausweitung der Produktion mit sich bringen (der Fall des reinen Lagerabbaus sei vernachlässigt), so daß ein Teil der staatlichen Ausgaben in Gestalt einer höheren Lohnsumme an die Arbeitnehmer fließt[135]. Sieht man von denkbaren Lohnsatzveränderungen ab, so wird die Produktionsfunktion sowie das unternehmerische Preissetzungskalkül für die Entwicklung der Verteilung maßgeblich, das rein nachfragetheoretische Verteilungsmodell kann diese nicht mehr eindeutig erklären.

Aber auch hier ist für das Erreichen einer Gleichgewichtssituation, in der die freiwilligen Konsumverzichte mit den gesamtwirtschaftlich notwendigen übereinstimmen, vonnöten, daß die zusätzliche staatliche Kreditnachfrage durch private Ersparnisbildung alimentiert wird. So wird in Abhängigkeit von der Zunahme der gesamten Wertschöpfung einerseits und der Lohnsumme andererseits auch die Ersparnisbildung der Arbeitnehmer zunehmen, aber ohne die oben erwähnten zusätzlichen Informationen können die sich dabei einstellenden Verteilungssituationen nicht eindeutig bestimmt werden[136]. Allerdings kann in einer solchen Aufschwungsphase schwerlich von einer stabilen Gleichgewichtssituation die Rede sein, da sich verschiedene Multiplikator- und Akzeleratorwirkungen überlagern werden.

Diese multiplikativen und akzelerativen Wirkungen sind nun im Gegensatz zur Situation der Vollbeschäftigung nicht mehr rein nominaler Natur, sondern sind mit Produktions- und Beschäftigungswirkungen verbunden, die für beide Gruppen eine absolut höhere Güterversorgung bewirken. Allerdings sind diese Niveaueffekte von Struktureffekten überlagert, denen aber vergleichsweise geringe verteilungspolitische Relevanz zukommt. Schon oben wurde auf die 
u.U. beschränkte Aussagekraft von Quoten verwiesen, da ein relatives Zurückbleiben bei gleichzeitig allgemein sich verbesserndem Verteilungsniveau einer differenzierten Beurteilung bedarf.

Im Rahmen eines solchen Aufschwungsprozesses, der von der zusätzlichen Staatsverschuldung nur initiiert wurde, mit einer zunehmenden Investitions quote und den daraus ableitbaren Erfordernissen an die gesamtwirtschaftliche Vermögensbildung ist allerdings damit zu rechnen, daß die Gewinnquote schneller zunehmen wird als die Arbeitnehmereinkommen, da sich diese zusätzlichen autonomen Ansprüche an das Sozialprodukt in Kreislaufgewinnen niederschlagen. So läßt sich auch empirisch beobachten, daß die Lohnquote in rezessiven Phasen ihre höchsten Werte aufweist[137], in einer Situation also, die meist auch durch ungenügende Investitionsgüternachfrage gekennzeichnet ist. Andererseits wird die in Aufschwungphasen steigende Gewinnquote (es sei nochmals an die Vermögenszusammenhänge erinnert) immer wieder ein lohnpolitisches und damit auch stabilitätspolitisches Problem sein, obwohl sie eine systembedingte Notwendigkeit darstellt. Wenn diese Entwicklung der Verteilungsquoten Ausdruck des systemimmanenten Prozesses zur Rückgewinnung von Stabilität und Vollbeschäftigung ist, kann man daran kein negatives verteilungspolitisches Urteil anknüpfen[138].

Stellt man das niveauorientierte Verteilungsziel, das mit dem der Vollbeschäftigung übereinstimmt, über das an formalen Quoten orientierte strukturelle, so wird die Vernachlässigung der Produktions- und Einkommenseffekte im Rahmen der Inzidenzüberlegungen durchaus plausibel. Bei einer solchen Prioritätssetzung rücken die strukturellen Überlegungen erst dann wieder in den Vordergrund, wenn es um die durch konkurrierende Ansprüche hervorgerufenen Verteilungsprobleme geht.

Wenn auch aufgrund des hier verwendeten theoretischen Erklärungsansatzes keine eindeutigen Aussagen zu den Verteilungswirkungen der Staatsverschuldung in der Rezession gemacht werden, sondern nur die Endpunkte eines durch eine solche Kreditnahme angestoßenen Aufschwungsprozesses aufgezeigt werden können, ist es doch von stabilitäts - und verteilungspolitischer Bedeutung, die innere Notwendigkeit der dabei eintretenden Entwicklung der Verteilungsquoten aufgezeigt zu haben. 


\section{B) NEOKLASSISCHER ANSATZ}

\section{Charakterlstik der Analyse}

Im Gegensatz zur Keynestanischen Erklärung der Vertellung, die die Nachfrageströme und ihre Veränderungen in den Mittelpunkt der Betrachtung stellt, gehen neoklassisch orientierte Ansätze von der Angebotsseite aus. Aus dem Zusammenspiel der streng rational operierenden Wirtschaftssubjekte auf (je nach Annahme) mehr oder weniger vollkommenen Märkten ergibt sich ein gesamtwirtschaftliches Verteilungsergebnis, das weitgehend in $\mathrm{Ab}-$ hängigkeit von technologischen Bestimmungsfaktoren zu sehen ist[139].

Unter der Annahme einer klassischen (linear-homogenen) Produktionsfunktion, der für alle Märkte geltenden Form der vollkommenen Konkurrenz und der Gewinnmaximierung als Verhaltensmaxime ist die gesamtwirtschaftliche Verteilung determiniert. Die mit ihren Grenzwertprodukten entlohnten Faktoren schöpfen das Volkseinkommen aus (adding-up-theorem), Gewinneinkommen sind nicht existent[140]. Wenn diese Annahmen auch nur das einfache Grundmodell umschreiben, entsprechen diese Wirkungsmechanismen doch denen modifizierter Ansätze; auch bei ihnen werden die Anteile der funktionellen Einkommensarten durch die Eigenschaften der Produktionsfunktion und die Marktformen bestimmt[141].

Diese für verteilungspolitische Zielsetzungen zunächst resignativ stimmende Aussage betrifft aber nur die funktionelle Einkommensverteilung, die hier enger gefaßt ist als bei der kreislauftheoretischen Betrachtung. Mit dem Aufzeigen der Entwicklung der funktionellen Einkommensverteilung ist aber noch keine Aussage über die primär interessierende institutionelle Verteilung verbunden. Hierzu müssen zusätzliche Informationen über die Verteilung der Bestandsgrößen bekannt sein, um eine Zuordnung der funktionellen Einkommensarten vornehmen zu können.

Trotz dieser Relativierung der unmittelbaren verteilungspolitischen Relevanz der funktionellen Einkommenskategorien im angebotstheoretischen Ansatz müssen diese doch Grundlage für alle Überlegungen zur personellen Einkommensverteilung sein. Wenn im folgenden die Distributionswirkungen der staatlichen Neuverschuldung im grenzproduktivitätstheoretischen Ansatz aufgezeigt werden sollen, bedeutet dies, daß unter Berücksichtigung der Einflüsse auf die relativen Seltenheiten der Produktionsfaktoren die tendenziellen Entwicklungen ihrer Preise (Zins - und Lohnsätze) sowie ihre relativen Anteile am Volks einkommen $\mathrm{zu}$ erfassen sind. Es ist allerdings zu bedenken, daß Anpassungs prozesse an veränderte Faktorpreis-Relationen wohl eher auf lange Sicht zu erwarten sind. Da dieser Einwand aber für die reine Modellwelt der vollkommenen Konkurrenz unerheblich ist und ohnehin nur die tendenziellen, modellimmanenten Entwicklungen verfolgt werden, sollen diese Wirkungen trotz des auf die kurzfristige Betrachtung abstellenden Untersuchungsziels aufgezeigt werden[142]. Darüber hinaus entbehrt die zeitliche Zuordnung der zu untersuchenden Effekte nicht einer gewissen Problematik. Im Vordergrund der Be- 
trachtung soll die Phase der Schuldaufnahme stehen, da zu diesem Zeltpunkt durch das staatliche Eingrelfen Wirkungen hervorgerufen werden Veränderungen der Zinssätze)[143], die sich bei Unterstellung einer entsprechend hohen Anpassungsgeschwindigkeit noch in dieser Perlode auf die relativen Seltenheiten der Faktoren Kapital und Arbeit auswirken.

2. Staatliche Schuldaufnahme als Bestandteil der gesamtwirtschaftlichen Vermögensbildung

Analog zum Vorgehen bei der kreislauftheoretischen Betrachtung soll untersucht werden, wie sich die Primärverteilung aufgrund einer staatlichen Kreditaufnahme verändert. Von wenigen Ausnahmen abgesehen wurden diese Effekte der Staatsverschuldung von den Autoren, die sich der klassischen Schuldtheorie bedienen, überhaupt nicht beachtet oder aber als vernachlässigbar dargestellt. Mit dem Erscheinen des Aufsatzes von Gandenberger zu den Verteilungswirkungen öffentlicher Kreditnahme[144] hat sich allerdings ein grundlegender Wandel im formalen Ansatz vollzogen, dessen Spuren deutlich in der Literatur zu verfolgen sind[145]. So findet sich in den meisten neueren finanzwissenschaftlichen Lehrbüchern zumindest ein Hinweis auf die Bedeutung der funktionellen Einkommensverteilung, wenn dies auch häufig als ein von der personellen Verteilung völlig unabhängiger Sachverhalt behandelt wird[146]. Gandenberger lenkte die Aufmerksamkeit vom Steuer-Zins-Kreislauf, der als der verteilungspolitisch relevanteste Sachverhalt in der klassischen Schuldtheorie galt, auf die Veränderungen der Primärverteilung durch eine Neuverschuldung. Damit wurde die unglückliche, vom Transfer-Ansatz unterstellte Kausalverbindung zwischen der Anleihefinanzierung und der von anderen Faktoren abhängigen Wahl des Anlageorts aufgelöst.

\section{Entwicklung des Faktorpreises Zins}

Im folgenden soll der Grundgedanke Gandenbergers aufgegriffen, dann aber im Rahmen der grenzproduktivitätstheoretischen Erklärung der Verteilung wei tergeführt werden. Steht bei Gandenberger die Erklärung der Verteilungssituation der Verzinsungsperiode im Vordergrund[147], so rechnen wir diese Effekte aus obengenannten Gründen bereits der Periode der Schuldaufnahme zu. Im nächsten Kapitel wird auch auf die Verteilungssituation der nachfolgenden Verzinsungsperiode eingegangen.

Gemäß der faktorpreisorientierten Sicht der Grenzproduktivitätstheorie der Verteilung muß das Geschehen auf dem Kapitalmarkt untersucht werden, um über dort stattfindende Preisveränderungen erste Rückschlüsse auf die Verteilungswirkungen zu ziehen. Die Konstanz der Kapitalangebotsfunktion, was der Annahme einer streng exogen determinierten Geldmenge gleichkommt[148], ist eine der wesentlichen Ausgangsprämissen der Ableitung Gandenbergers. Von der Pufferfunktion des Bankensystems wird also abstrahiert; die gesamt- 
wirtschaftlich zur Verfügung stehende Kreditmenge ist ausschließlich von der privaten Ersparnisbildung abhängig. Die zusätzliche, staatliche Kapitalnachfrage kommt in einer Verschiebung der gesamtwirtschaftlichen Nachfragefunktion zum Ausdruck. Da auch Gandenberger von einer völlig zinsunelastischen Nachfrage des Staates ausgeht, erfährt die Nachfragefunktion eine Parallelverschiebung um den Absolutbetrag der staatlichen Kreditwünsche nach rechts[149]. Bei konstanter Angebotsfunktion, und dies in Frage zu stellen gibt die Staatsverschuldung keinen Anlaß[150], wird sich ein neues Marktgleichgewicht einstellen, dessen Lage - und damit der neue Gleichgewichts zinssatz und die Kreditmenge - im Vergleich zum alten Gleichgewichtspunkt von den Elastizitäten der jeweiligen Funktionen abhängt. Wir wollen dabei von den Extremen völlig elastischer bzw, unelastischer Verläufe absehen, sind doch die klassischen Funktionen durch dazwischen liegende Elastizitätswerte charakterisiert[151]. Der hier gewählte Verlauf für Angebots - und Nachfragefunktion unterstellt also sowohl für die Ersparnisse als auch die Investitionen Zinsabhängigkeit.

In folgender Skizze sei NN die gesamtwirtschaftliche Kreditnachfragefunktion, die Kurve AA die entsprechende Angebotsfunktion. Die Nachfrage verschiebt sich nun im Umfang von $K_{S t}$ nach rechts in die Lage $N^{\prime} N^{\prime}$, wodurch sich der neue Gleichgewichtspunkt $S_{2}$ mit den Koordinaten $i_{2}$ und $K_{2}$ ergibt.

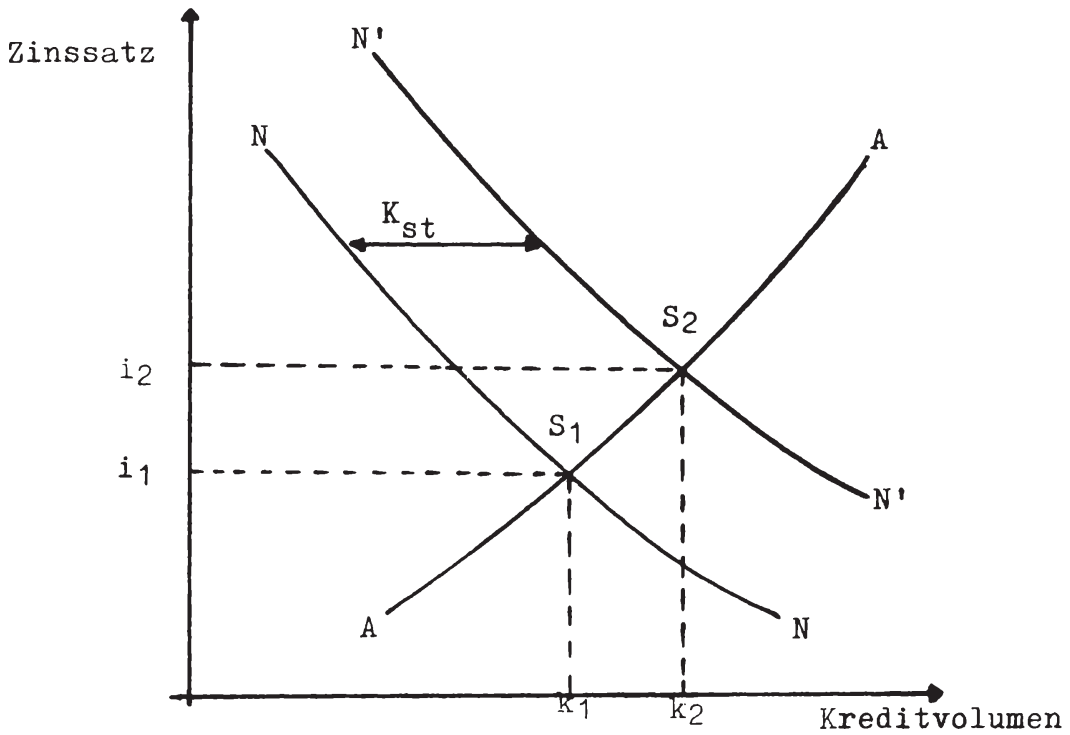


Für die Entwicklung des Zinssatzes einerseits und der sich aus Menge und Preis zusammensetzenden Zinssumme andererseits lassen sich einige generelle Abhängigkeiten aufzeigen. Bei gegebener Nachfragefunktion wird der Verdrängungseffekt privater Investitionen um so geringer, je zinselastischer die Angebotsfunktion ist, bei einem um so größeren Kreditvolumen wird auch der neue Gleichgewichtspunkt $\mathrm{S}_{2}$ liegen. Hier nimmt die Vermögensneubildung am stärksten zu, die Zinssatzerhöhung fällt dagegen nicht so stark aus.

Je zinsunelastischer dagegen die private Ersparnisbildung reagiert, desto höher müssen die Zinssätze steigen, um die private Investitionsnachfrage vom Markt zu verdrängen. Für das Ausmaß dieser notwendigen Zinssatzsteigerung ist aber jeweils auch die Zinselastizität der privaten Investitionsgüternachfrage maßgebend.

Die Entwicklung dieser Einzelfaktoren (Preis - und Mengeneffekte) ist für die Interpretation der Verteilungswirkungen von Bedeutung. Bei Auftreten von Kreditmengeneffekten nimmt die Vermögensbildung $z u$, damit wird die Frage nach dem Personenkreis aufgeworfen, der zusätzliches Vermögen bildet[152]. Darüber hinaus muß auch die Verteilung der Bestände bekannt sein, da von Zinssatzeffekten alle Kapitalbesitzer profitieren. Die Betrachtung kann sich also nicht nur auf die Entwicklung des Aggregats der Zinseinkommen beschränken, da der Differenzierung nach Preis- und Mengeneffekten für über die rein funktionelle Verteilung hinausgehende Aussagen erhebliche Bedeutung zukommt.

4. Wirkungen auf die funktionelle Einkommensverteilung

Spätestens an dieser Stelle muß der ceteris-paribus-Charakter der Betrachtung betont werden. Die Entwicklung der Zinssumme und -quote ist nicht unabhängig von den Reaktionen des Faktoreinsatzverhältnisses und der jeweiligen Faktorpreise zu sehen. Gandenberger betrachtet in seiner Analyse die unmittelbaren Einflüsse der staatlichen Nachfrage auf einem bestimmten Markt, verfolgt weitergehende Wirkungen aber nicht.

Um zu einer vollständigeren Beurteilung der Verteilungseffekte im grenzproduktivitätsorientierten Kalkül zu kommen, muß die Entwicklung der anderen funktionellen Einkommensart hinterfragt werden. Im hochaggregierten makroökonomischen Modell wird dies der Preis für den Faktor Arbeit sein; es muß also zusätzlich die Entwicklung der Lohnsätze beachtet werden. Der Rationalität dieses Ansatzes zufolge ziehen Veränderungen der Faktorpreise unmittelbar solche im Einsatzverhältnis dieser Faktoren im Produktionsbereich nach sich. Die durch den exogenen Impuls des Staates bewirkte Zinssatzerhöhung bedeutet eine Verteuerung des Faktors Kapital, was einen verstärkten Einsatz des substitutiven Faktors Arbeit mit sich bringt. Bei linear-homogenen Produktionsfunktionen mit einer Substitutionselastizität von eins, und eine solche wurde bisher unterstellt, ergibt sich bei einer Änderung der Zins/Lohnrelation eine diesen Effekt kompensierende Veränderung im Kapital/Arbeits-Einsatzverhältnis. Dadurch bleibt die funktionelle Einkommensverteilung konstant, auch wenn 
die Preisrelation zwischen Zins - und Lohnsatz eine Änderung erfährt. Mit diesem Umstand begründet Gandenberger auch, daß er seine Überlegungen nicht in den Rahmen einer weitergehenden Verteilungsanalyse stellt[153].

Dies ist aber nur dann haltbar, wenn tatsächlich eine Substitutionselastizität von eins anzunehmen ist. Weist die gesamtwirtschaftliche Produktionsfunktion andere Werte auf, so sind natürlich die daraus resultierenden Änderungen der funktionellen Einkommensverteilung zu beachten.

Bleiben wir aber beim Fall einer Konstanz der funktionellen Verteilung, so ist auch hier vor vorschnellen verteilungspolitischen Schlußfolgerungen zu warnen. Bei Einbeziehung des Staates sind auch dessen Besitzeinkommen in der entsprechenden gesamtwirtschaftlichen Quote enthalten. Aufgrund der Kreditaufnahme geht aber der Anteil des Staates an dieser funktionellen Einkommensart zurück bzw. wird negativ. Damit steigt bei gesamtwirtschaftlich konstanter Besitzeinkommensquote die des privaten Sektors.

Bleibt man bei Betrachtung der funktionellen Einkommensverteilung stehen, so ergeben sich also keine verteilungsrelevanten Effekte. Berücksichtigt man aber zusätzlich die Antelle des privaten und des öffentlichen Sektors, so ergeben sich in der Schuldaufnahmephase bei konstanter Quote des Arbeitseinkommens eine Erhöhung der Quote des privaten Besitzeinkommens und eine entsprechende Verminderung der Quote des staatlichen Sektors.

\section{Wirkungen auf die institutionelle Verteilung}

Die verteilungspolitische Ziel- und Maßstabsgröße kann auch innerhalb dieses Ansatzes nicht die funktionelle, sondern nur die institutionelle Einkommensverteilung sein. Die erste Differenzierung dieser Art wurde mit Berücksichtigung der staatlichen Besitzeinkommensquote bereits vorgenommen. Aber auch für den privaten Sektor könnten unmittelbar verteilungspolitisch verwertbare Aussagen nur dann abgeleitet werden, wenn in diesem Sektor keinerlei Querverteilung bestünde, d.h. personelle und funktionelle Einkommensverteilung identisch wären.

Verwirft man diese Annahme aber als unrealistisch, so ist zunächst nach dem jeweiligen Anteil der Zinseinkommen am Gesamteinkommen einer bestimmten Gruppe zu fragen, da alle Empfänger dieser Einkommensart bei Zinssatzsteigerungen ein absolut höheres Zinseinkommen beziehen werden. Auch hier weitet sich also die Sicht von den Zeichnern der staatlichen Anleihe auf alle Vermögensbildenden dieser Periode, auch im klassischen Ansatz muß die Staatsverschuldung als Bestandteil der allgemeinen Vermögensbildung aufgefaßt werden, will man ihre effektive Inzidenz ermitteln.

Neben dem Zinssatzeffekt ist aber auch in Abhängigkeit von den Zinselastizitäten ein Kreditmengeneffekt $z u$ verzeichnen. Wenn eine zusätzliche Vermögens blldung stattfindet, ist also zu fragen, wo sich diese Vermögensbildung niederschlägt, well auch hiervon Auswirkungen auf das Pro-Kopf-Einkommen und damit die personelle Verteilung zu erwarten sind. 
Selbst bei Annahme einer Produktionsfunktion mit der Substitutionselastizität von eins ergibt sich im grenzproduktivitätstheoretischen Ansatz eine Veränderung der Einkommensverteilung der funktionellen Einkommensarten für den privaten Sektor, da dort die Besitzeinkommensquote gestiegen ist. Nur bei Nichtberücksichtigung dieser Struktureffekte kann man zum Ergebnis kommen, daß die Verteilungssituation aufgrund der Konstanz der funktionellen Einkommensverteilung unberührt geblieben sei.

Legt man dagegen Produktionsfunktionen mit Substitutionselastizitäten ungleich eins zugrunde, so könnte sich hypothetisch für den privaten Sektor auch eine Konstanz der Einkommensverteilung ergeben. Für eine differenziertere verteilungspolitische Beurteilung müssen aber auch dann zusätzliche Informationen über die Besitzverhältnisse an den Bestandsgrößen vorhanden sein, da nur so eine Aussage iuber die institutionelle Verteilung möglich ist.

Vergleicht man die Verteilungswirkungen der staatlichen Schuldaufnahme in grenzproduktivitätstheoretischer Interpretation mit den oben aufgezeigten Ergebnissen, so zeigt sich ein nicht zu vernachlässigender Unterschied. Bei kreislauftheoretischer Betrachtung der von der klassischen Theorie unterstellten Wirkungsmechanismen ergab sich nämlich, daß für die institutionelle Verteilung keine Veränderungen zu erwarten sind. Dies kann für die grenzproduktivitätstheoretische Verteilungserklärung zwar auch eintreten, muß aber als konstruierter Grenzfall bezeichnet werden.

Dieser Vergleich zwischen den beiden Erklärungsansätzen soll aber hier nicht weiter getrieben werden, da völlig unterschiedliche Einkommenskategorien erfaßt werden. Hierin ist gleichzeitig der Haupteinwand gegenüber der grenzproduktivitätstheoretischen Erklärung der Verteilung zu sehen, daß sie nämlich die in Verbindung mit einer staatlichen Kreditnahme konstitutiven Kreislaufbeziehungen nicht berücksichtigt und die daraus resultierenden Kreislaufprofite ïberhaupt nicht erklären kann.

Für die Erklärungsdominanz des kreislauftheoretischen Ansatzes spricht vor allem der Umstand, daß durch die zusätzliche Nachfrage des Staates immer zumindest in gewissem Umfang expansive Effekte auf die gesamtwirtschaftliche Nachfrage ausgehen werden. Dann aber wird eine verteilungstheoretische Erklärung unbrauchbar, die solche Vorgänge iberhaupt nicht erfassen kann.

Dennoch erschien es sinnvoll, die grenzproduktivitätstheoretischen Überlegungen für eine staatliche Schuldaufnahme in Anlehnung an Gandenberger aufzugreifen, da die entscheidende Gemeinsamkeit im methodischen Ansatz besteht. Auch die klassische Schuldtheorie muß zur Erklärung der effektiven Inzidenz der Staatsverschuldung auf die grundlegenden, verteilungsdeterminierenden Mechanismen und thre Beeinflussung durch die staatliche Schuldaufnahme Bezug nehmen. Bei modellimmanenter Argumentation ergab sich dabel, daß durchaus verteilungspolitisch bedeutsame Sachverhalte zu beachten sein werden, wenn auch keine unmittelbar auf verteilungs relevante Gruppierungen be- 
zogene Aussagen möglich sind. So liegt also auch in der direkten Ausrichtung auf die institutionelle Verteilung ein Vorteil der kreislauftheoretischen Erklärung, dem aber im Vergleich zum oben genannten konstitutiven Mangel des neoklassischen Erklärungsansatzes der Verteilung nur untergeordnete $\mathrm{Be}-$ deutung zukommt. 
Viertes Kapitel

\author{
VERTEILUNGSEFFEKTE IN DER PHASE DES SCHULDEN- \\ ST A N E ES
}

\title{
A) AUSWIRKUNGEN DER SCHULDAUFNAHME AUF DIE FOLGEPERIODE
}

1. Kreislauftheoretischer Ansatz

\section{a) Stationäre Wirtschaft}

Die bisherigen Überlegungen zu den Verteilungswirkungen einer staatlichen Kreditaufnahme betrafen die Phase der Schuldaufnahme. Dabei stand ausschließlich die Primärverteilung zur Diskussion, da der Staat nur über die marktmäßige Schuldaufnahme tätig wurde. Bevor die für die Phase des Schuldenstandes zentralen Effekte der Verzinsung auf die Sekundärverteilung untersucht werden, soll dem eine Betrachtung der Nachwirkungen der Schuldaufnahme auf die Einkommensverteilung der folgenden Periode vorausgehen. Dies berührt insofern den Problemkreis der Verzinsung, als die Primärverteilung dieser Periode Ausgangsbasis der Sekundärverteilung ist. Dabei soll zunächst eine stationäre Wirtschaft unterstellt sein, d.h. die Kapazitätseffekte der Nettoinvestitionen werden vernachlässigt, da so im ersten Schritt mit Absolutgrößen argumentiert werden kann.

Wir kamen oben zum Schluß, daß sich die autonomen Ansprüche des Staates bei hinreichender Elastizität des Bankensystems über Preiseffekte durchsetzen werden. Da es sich bei dieser Kreditaufnahme den Annahmen entsprechend um einen einmaligen Vorgang handelte, ergibt sich in der Folgeperiode ein Rückgang der staatlichen Ansprüche auf das Nullniveau der der Schuldaufnahme vorangehenden Periode. Dann wird sich auch die Einkommensverteilung in ceteris-paribus-Betrachtung auf diesem Ausgangsniveau einspielen, die angefallenen Kreislaufgewinne werden wieder aufgezehrt. Es wird also die Verteilungssituation wiederhergestellt, die vor dem Eintreten des exogenen staatlichen Impulses gegeben war[154].

Behält der Staat hingegen in der Folgeperiode in gleichem Umfange kreditfinanzierte Ansprüche an das Sozialprodukt bei, so ergibt sich c.p. keine neue Verteilungsänderung; die für die Vorperiode aufgezeigten Verteilungswirkungen bleiben bestehen. Dies ist unmittelbar einsichtig, wenn man sich den verteilungsdeterminierenden Charakter der Nachfrageimpulse vor Augen hält; der Staat hat seinen Anteil bereits in der Vorperiode durchgesetzt, neue Preisund damit Verteilungseffekte sind aber nur bei zusätzlichen autonomen Ansprüchen an das Sozialprodukt zu erwarten. 
Die Unterstellung einer stationären Wirtschaft stellt eine sehr restriktive Annahme dar. Deswegen soll nun auf einen Fall mit Wirtschaftswachstum abgestellt werden. Die Ansprüche an das Sozialprodukt, die die Verwendungsstruktur einer bestimmten Periode charakterisieren, lassen sich durch Interpretation der einzelnen Größen als Quoten am Sozialprodukt erfassen[155]. Geht aber, wie hier angenommen, die Neuverschuldung des Staates auf Null zurück, so steht bei Konstanz der Verwendungsstruktur im privaten Bereich ein gütermäßiger Anteil am Sozialprodukt zur Verfügung, der sich in Verteilungsänderungen zugunsten der Arbeitnehmer niederschlägt. Auch in einer wachsenden Wirtschaft ergibt sich so eine Rückkehr zur Verteilungssituation der der Schuldaufnahme vorhergehenden Periode, wobei dies in den alten Verteilungsquoten zum Ausdruck kommt.

Es ist allerdings auf die Grenzen einer solchen ceteris-paribus-Argumentation zu verweisen, die eine Konstanz aller anderen Quoten der Vorperiode Impliziert. Dies stellt eine nicht unproblematische Annahme dar, da sich die Ansprüche der Wirtschaftssubjekte und des Staates an Absolutgrößen orientieren werden. Oben wurde schon in Zweifel gezogen, daß etwa eine Kreditquote Zielgröße für das staatliche Haushaltsgebaren sein könne. Für den Fall der einmaligen Kreditaufnahme spielen diese Überlegungen keine Rolle, hier stellt sich c.p. (wie auch im Falle der stationären Wirtschaft) die strukturelle Verteilung der Periode vor der staatlichen Schuldaufnahme wieder ein. Hält der Staat dagegen seine Kreditnahme in absoluter Höhe aufrecht, so bedeutet dies bei Annahme von realem Wachstum eine Reduktion der staatlichen Schuldaufnahmequote, damit aber auch eine teilweise Kompensation der in der Schuldaufnahmephase erzwungenen Verteilungsänderungen.

Umgekehrt formuliert sind in den Folgeperioden nur dann Preiseffekte infolge einer staatlichen Neuverschuldung zu erwarten, wenn bei Konstanz der anderen Aggregate der gesamtwirtschaftlichen Verwendungsstruktur der relative Anteil der staatlichen Kreditnahme am Sozialprodukt der betrachteten Periode uiber den entsprechenden Wert der Vorperiode hinausgeht. Aus Gleichung III. 15 ergibt sich, daß bei Konstanz der anderen Quoten lediglich eine Erhöhung der Kreditquote (absolute Neuverschuldung bezogen auf das Sozialprodukt) gegenüber der des Vorjahres weitere Verteilungsänderungen hervorzurufen vermag:

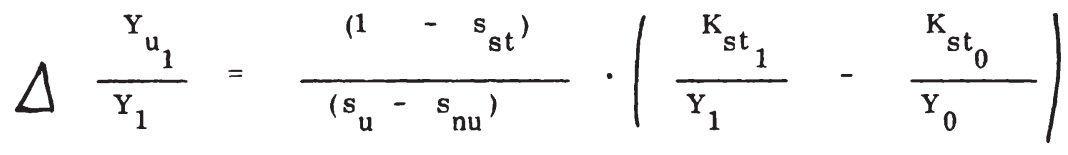

Löst man die ceteris-paribus-Klausel auf, da die Annahme einer konstanten Verwendungsstruktur ohnehin nicht sonderlich plausibel erscheint, so sind die dadurch entstehenden Bewegungsspielräume bzw. Restriktionen zusätzlich zu beachten. Entscheidender Bezugspunkt für die verteilungspolitische Beurteilung 
einer staatlichen Neuverschuldung muß also die gesamtwirtschaftliche Verwendungs-bzw. Nachfragestruktur und deren Veränderungen im Vergleich zur Vorperiode sein.

Diese Überlegungen stellen eine Erweiterung der eigentlichen Fragestellung dar, da von einer einmaligen Verschuldung ausgegangen wurde und deren Verteilungseffekte in den folgenden Perioden aufgezeigt werden sollten. Hierfür kommt man zur eindeutigen Antwort, daß die Verteilungseffekte einen einmaligen Impuls auf die Einkommensverteilung darstellen, mit Fortfall dieses Impulses aber c.p. auch die Einkommensverteilung auf ihr altes Niveau zurückkehrt[156]. Die modifizierenden Überlegungen sind aber dadurch zu rechtfertigen, daß in Kapitel III. vom erstmaligen Auftreten des Staates ausgegangen wurde, damit aber der Umstand einer staatlichen Schuldaufnahme der Vorperiode überhaupt nicht in das Verteilungskalkül mit eingehen konnte. In einem weniger abstrakten Fall sind obige Überlegungen zur Beurteilung der konkreten Verteilungswirkungen einer staatlichen Verschuldung aber zusätzlich zu berücksichtigen.

\section{Grenzproduktivitätstheoretischer Ansatz}

Auch in diesem Abschnitt soll davon ausgegangen werden, daß die staatliche, kreditfinanzierte Nachfrage in der ersten Verzinsungsperiode auf das alte Nullniveau zurückkehrt. Die oben in grenzproduktivitätstheoretischer Sicht aufgezeigten Verteilungswirkungen der Staats verschuldung kamen dadurch zustande, daß der Staat auf Ressourcen zurückgriff, die andernfalls in die private Kapitalbildung eingegangen wären. Durch diese staatliche Absorption wurden die relativen Seltenheiten verändert. Der private Kapitalstock bleibt dadurch c.p. auch in den Folgeperioden geringer als im Falle ohne staatliches Eingreifen[157]. Entscheidend für diesen Ansatz ist, daß die in der Phase der Schuldaufnahme bewirkten Veränderungen auch auf die Einkommensverteilung der Folgeperioden Einfluß ausüben.

Bei der Betrachtung der Schuldaufnahmephase wurde auf die Abhängigkeit der funktionellen Einkommensverteilung, die den Ausgangspunkt für weitere Verteilungsaussagen darstellt, von den Substitutionselastizitäten der zu unterstellenden gesamtwirtschaftlichen Produktionsfunktion hingewiesen. Unabhängig von den sich ergebenden (im Falle der Substitutionselastizitäten $\neq 1$ ) oder nicht ergebenden (Substitutionselastizität $=1$ ) Änderungen der funktionellen Einkommensverteilung ist der entscheidende Unterschied zum kreislauftheoretischen Ansatz darin zu sehen, daß diese Veränderungen der Primärverteilung der Schuldaufnahmephase aufgrund geänderter relativer Seltenheiten in der Folgeperiode Bestand haben. Allerdings nimmt der Einfluß eines solchen einmaligen, exogenen Impulses bei Annahme einer wachsenden Wirtschaft ab und wird bei langfristiger Betrachtung den Wert Null erreichen. 
Bei der kreislauftheoretischen Betrachtung ergab sich, daß die Primärverteilung der Schuldaufnahmephase in der nachfolgenden Periode auf ihr Ausgangsniveau zurückkehrt, wenn keine weitere Schuldaufnahme erfolgt. Die strukturellen Verteilungseffekte sind an die unmittelbare Verausgabung geknüpft, haben aber keinen Einfluß auf die Verteilung in der Folgeperiode. Dies ergibt sich aus den unterschiedlichen Mechanismen der Verteilungserklärung. Ist für die kreislauftheoretische Betrachtung nur der zusätzliche, nachfrageerweiternde Effekt maßgeblich, so sind dies im klassischen System die durch den exogenen staatlichen Einfluß bewirkten Änderungen der relativen Seltenheiten, die auch nach Wegfall des Impulses - allerdings immer geringer werdende - Auswirkungen zeigen.

B) ZINSZAHLUNGEN AUF STAATSPAPIERE

1. Traditionelle Sicht

a) Vorbemerkung

Oben wurde die Sekundärverteilung als die Verteilung charakterisiert, die die staatliche Eingriffe in Form der Erhebung von Steuern und deren Verausgabung erfaßt. Da wir die staatliche Aktivität lediglich in Form einer einmaligen Schuldaufnahme berücksichtigen, waren in der ersten Phase noch keine für die Sekundärverteilung relevanten Effekte zu beachten.

In der Verzinsungsperiode treten nun aber die Finanzierung der Zinszahlungen des Staates und ihre Transferierung in den privaten Sektor als Phänomene der Sekundärverteilung auf. Diese Zahlungsströme stellen demnach, ungeachtet ihres Charakters als Vermögenseinkommen für die privaten Haushalte, Einkommen dar, die das Verteilungsbild der die gesamtwirtschaftliche Wertschöpfung umfassenden Primärverteilung noch einmal verändern können[158].

Dem kreislauftheoretischen Konzept entsprechend ist für die effektive Inzidenz der Zinssteuern - und ausschließlich von einer Steuerfinanzierung der Zinsen soll hier ausgegangen werden - von Bedeutung, in welcher Weise die privaten Wirtschaftssubjekte die Zinszahlungen verwenden; denn es ist für die Verteilungswirkungen ausschlaggebend, wie die Nachfrageströme durch die sekundäre Verteilung beeinflußt, d.h. verengt oder erweitert werden. In diesem Sinne wird im folgenden die effektive Inzidenz der Zinssteuern untersucht, die rein formale Ähnlichkeit mit dem überkommenen Transferansatz darf über die eigentliche Kausalität nicht hinwegtäuschen. 


\section{b) Transfer-Ansatz}

Nicht zuletzt unter dem Einfluß der klassischen Sichtweise galt und gilt teilweise heute noch das Hauptaugenmerk verteilungspolitischer Betrachtungen zur Staatsverschuldung der Phase des Schuldenstandes. Um zu verteilungspolitischen Aussagen bezüglich der Zinszahlungen zu gelangen, wird dabei die Verteilung der staatlichen Wertpapiere (und damit der Zinszahlungen) auf einzelne Einkommensklassen mit der jeweiligen Belastung eben dieser Einkommensklassen durch den Zinsendienst verglichen. Je stärker sich diese Verteilungen in ihren Relationen annähern, als desto "neutraler" wird die Verteilungswirkung charakterisiert. Verteilungspolitisch besonders positiv wird entsprechend dieser Argumentation der Fall bewertet, in dem die Staatspapiere von einkommensschwächeren Gruppen gehalten werden, diese aber keinen oder nur einen geringen Anteil der Zinssteuern aufbringen müssen. Je nach Progressivität oder Regressivität der beiden Strukturen und ihrer Kombinationen mitei nander ergibt sich ein mehr oder weniger wünschenswertes verteilungspolitisches Ergebnis, das sich im Nettotransfer manifestiert. Betrachtet man einmal die Aufbringungsseite als gegeben, so muß man zu dem erstaunlichen Ergebnis gelangen, daß allein durch eine Umschichtung des Vermögensbestandes zwischen einzelnen Einkommensgruppen Verteilungseffekte erzielbar sein sollen. Tauschen beispielsweise die Anleger in unteren Einkommensschichten ihre Industriepapiere gegen bisher von den oberen Einkommensgruppen gehaltene staatliche Papiere, so ergibt sich das nach Andel absurde Ergebnis[159], daß ein solcher Aktivtausch zwischen zwei Einkommensgruppen eine verteilungspolitisch vorteilhafte Aktion sein soll.

Unmittelbarer Ausfluß solcher Überlegungen sind die empirischen Untersuchungen zu den Verteilungswirkungen der Staatsverschuldung, die auf diese beiden Strukturen abstellen und daran eine verteilungspolitische Beurteilung anknüpfen[160]. Ein gerne angeführtes Vorgehen dieser $\operatorname{Art[161]}$ stellen auch die Empfehlungen des Wissenschaftlichen Beirats beim Bundeswirtschaftsministerium aus dem Jahre 1968 dar, der für eine verstärkte Anlage der Ersparnisse unterer Einkommensschichten in Staatspapieren plädierte[162]. Auch bei einer Reihe anderer Autoren finden sich immer noch derartige auf dem Transferansatz basierende Gedankengänge[163].

\section{c) Methodische Einwände}

Einer der entschiedensten Gegner dieses eben geschilderten Transferansatzes ist Gandenberger, der in seinem oben genannten Aufsatz die Aufmerksamkeit auf die funktionelle Einkommensverteilung lenkte. Sein Haupteinwand gegenüber einer Betrachtungsweise, die rein auf die Phase der Verzinsung abstellt, liegt darin, daß nicht untersucht wird, welche Rückwir kungen der Tatbestand der Schuldaufnahme auf die Verteilungssituation jener Periode mit sich brachte. Die Aussagen des Transferansatzes implizieren, 
daß sich mit Ausnahme der staatlichen Zinszahlungen und der dafür notwendigen Steuern keine verteilungsrelevanten Effekte ergeben. Der Vergleich unterschiedlicher Besitzstrukturen staatlicher Papiere und die daraus gezogenen verteilungspolitischen Schlußfolgerungen basieren auf dem Versäumnis, die relevante Alternative zu beachten. In rein formalizinidenztheoretischer Sicht werden verteilungspolitische Schlüsse gezogen, ohne zu fragen, welche Reaktionen auf die Finanzierungsmaßnahme erfolgten.

Um die unzulängliche Kausalitätsbeziehung zwischen Steuererhebung einerseits und Zinszahlungen andererseits aufzuzeigen, sei auf die individuelle Entscheidungssituation der Wirtschaftssubjekte in der Phase der Schuldaufnahme eingegangen.

Die Entscheidungen bezüglich des Konsum - und Sparverhaltens werden in jeder Periode aufgrund der zu diesem Zeitpunkt gerade gültigen Datenkonstellation gefällt. Das Sparmotiv (oder die Neigung zur Vermögensbildung) stellt eine originäre Art der Einkommensverwendung dar. Eine unmittelbare Kausalität zwischen dem Verhalten der Wirtschaftssubjekte und einer staatlichen Schuldaufnahme wäre nur dann gegeben, wenn diese wegen der staatlichen Kreditwünsche zusätzliche Ersparnis bilden würden. Wählt der Staat zur Mittelbeschaffung eine Kreditaufnahme, so hat dies für die Wirtschaftssubjekte aber keine unmittelbar erkennbaren Konsequenzen[164]. Diese passive Sichtweise vom einzelnen Wirtschaftssubjekt dürfte realistisch sein, da das Individuum nicht in den Entscheidungsprozeß über verschiedene Finanzierungsmaßnahmen eingreifen kann[165].

Wenn in späteren Perioden zur Finanzierung der Zinsverpflichtungen des Staates Steuern erhoben werden, wird auch zwischen diesen Sachverhalten für das Individuum keine Kausalität ersichtlich. Sowohl die Sparer allgemein als auch die Besitzer staatlicher Papiere erhalten Zinsen, weil sie zur Vermögensbildung beigetragen haben, nicht aber weil sich der Staat verschuldet hat. Geht man davon aus, daß die Wahl des Anlageorts im Vergleich zum generellen Sparentschluß zweitrangig ist, erscheint es auch von der Anlageentscheidung her nicht gerechtfertigt, bestimmte Teile der Einkommen aus Vermögen mit den Steuerzahlungen zu konfrontieren. Diese Überlegungen sind unabhängig davon, ob neues Vermögen gebildet wurde oder nicht. Wie oben gezeigt wurde, kann sowohl bei klassischen als auch bei keynesianischen Annahmen die gesamtwirtschaftliche Vermögensbildung zunehmen, was entsprechende Rückwirkungen auf die personelle Einkommensverteilung hat. Keinesfalls können aber die Verteilungseffekte mit der Methode des Transferansatzes erfaßt werden, da die Änderungen der Primärverteilung von der individuell gewählten Anlageform unabhängig sind. 
Werden aufgrund der staatlichen Kreditaufnahme freiwillig zusätzliche Ersparnisse getätigt, so ist auch dies noch keine Legitimation für die Betrachtungs weise des Transferansatzes. Im Unterschied zum Normalfall einer Anlagensubstitution ist hier jedoch eine weitere Größe mit ihren Auswirkungen auf die Primärverteilung der Verschuldungsperiode zu beachten. Auch dieser Fall einer zurechenbaren Beziehung zwischen der Anlage in staatlichen Papieren und den Zinszahlungen ändert nichts am Grundtatbestand, daß es sich dabei um eine Folge der Vermögensbildung handelt. An diesem Beispiel wird die Bedeutung der Abgrenzung von Zwangsanleihen deutlich, bei denen andere Motive als die der reinen Vermögensanlage für die Zeichnung verantwortlich sein können, man denke nur an gesamtwirtschaftliche Notstandssituationen[166].

d) Verteilungsrelevanz des Steuer-Zins-Kreislaufs für eine kreislauftheoretische Analyse

Wenn aus dem Saldo der Zinszahlungen und der Steuern zur Finanzierung eben dieser Zahlungen auch nicht die primären Verteilungseffekte der Staatsverschuldung abgelesen werden können, ist dieser Kreislauf doch ein verteilungsrelevantes Phänomen. Man muß hier Wittmann zustimmen, der Gandenberger die völlige Vernachlässigung dieses Sachverhalts vorhält[167]. Wir betrachten aber nur die Steuererhebung als kausal mit der Staatsverschuldung verbunden, da die Zinsen eine Folge der Vermögensbildung der Vorperiode darstellen, also auch bei einer anderen Anlageform Einkommensbestandteile der privaten Anleger wären[168].

Wenn im folgenden die Wirkungen der Zinssteuern auf die Sekundärverteilung, d.h. deren effektive Inzidenz, in kreislauftheoretischer Sicht ermittelt werden, handelt es sich somit nur scheinbar um ein Vorgehen gemäß dem Transferansatz. Wir konfrontieren Zinssteuerzahler und Zinsempfänger nicht der Kausalität wegen, sondern weil die gruppenspezifischen Effekte der Aufbringung und Verwendung dieser Zahlungsströme verteilungsrelevant sein können[169]. Auf diesen Aspekt des Steuer-Zins-Kreislaufs wurde schon früher vereinzelt hingewiesen, wenn damit auch meist eine konjunktur-oder wachstumspolitische Beurteilung verbunden war[170].

Dabei ist zu betonen, daß die dieser Analyse als Ausgangsgröße zugrundegelegte Primärverteilung in der Phase des Schuldenstandes die in der Vorperiode erfolgte Schuldaufnahme und ihre Konsequenzen für die Verteilung dieser Periode umschließt. Damit ist Gandenbergers Einwand Genüge getan, auch die Auswirkungen der Schuldaufnahme auf die Einkommensverteilung der Folgeperiode $\mathrm{zu}$ erfassen.

In dieser Primärverteilung sind auch all die anderen Zinseinkommen enthalten, die aus Vermögensbildung - mit Ausnahme der Anlage in staatlichen Papieren resultieren. Der Verdienst der Gandenbergerschen Sicht ist darin zu sehen, 
die Einseitigkeit des Transfer-Ansatzes nachgewiesen zu haben. Die staatliche Verschuldung darf nicht in partialanalytischer Verabsolutierung untersucht, sondern muß als Bestandteil der gesamtwirtschaftlichen Vermögensbildung begriffen werden. Darüber darf jedoch nicht vernachlässigt werden, daß aus dem speziellen Kreislauf der Sekundärverteilung unterschiedliche Verteilungseffekte resultieren können. Der entscheidende Punkt für eine Analyse der Verteilungswirkungen staatlicher Schuldaufnahme ist, daß sowohl die Rückwirkungen auf die Einkommensverteilung der ersten Periode als auch die auf die Sekundärverteilung der Folgeperioden und deren Interdependenz erkannt wird. Betrachtet man nur die Sekundärverteilung als ein verteilungspolitisches Problem, so resultiert aus der Vernachlässigung der Wirkungen der Schuldaufnahmephase eine verteilungspolitisch unvollständige Sicht.

2. Effektive Inzidenz der Zinssteuern

a) Zielsetzung der Analyse

Der folgende Abschnitt untersucht die effektive Inzidenz der Zinssteuern in kreislauftheoretischer Sicht, wobei aufgrund des Untersuchungsansatzes für. die Verteilungswirkungen die Verwendung dieser Mittel von ausschlaggebender Bedeutung ist. Wenn der Steuer-Zins-Kreislauf auch aufgrund der für die Schuldaufnahme abgeleiteten Ergebnisse nicht die wesentlichen Verteilungswirkungen der Staatsverschuldung ausmachen kann, so ist doch zu überprüfen, welche unterschiedlichen Effekte mit bestimmten Besitz- und Aufbringungsstrukturen verbunden sind. Dem kreislauftheoretischen Ansatz entsprechend wird dabei auf die Positionen der Gruppe der Unternehmer einerseits und der Arbeitnehmer andererseits abgestellt.

Es sollen im folgenden also die von der Verzinsung der staatlichen Papiere ausgehenden strukturellen Verteilungseffekte dargestellt werden. Dabei untersuchen wir stellvertretend für alle kommenden Verzinsungsperioden[171] die unmittelbar der Schuldaufnahme folgende. Dies geschieht, wie schon betont, um so auch die Auswirkungen zu erfassen, die von der Schuldaufnahme im eigentlichen Sinne auf die Einkommensverteilung dieser ersten Verzinsungsperiode ausstrahlen. Hierbei zeigten sich erhebliche Unterschiede zwischen der klassischen und der kreislauftheoretischen Betrachtung, wobei nach dem kreislauftheoretischen Ansatz keine Auswirkungen mehr von der Schuldaufnahme zu erwarten sind. Von diesem Zwischenergebnis ausgehend sollen nun nur noch die von der Verzinsung herrührenden Effekte untersucht werden. Hierbei stcht die Entwicklung der Sekundärverteilung, d.h. die Inzidenz der Zinssteuer, im Vordergrund, doch müssen auch immer die durch diesen sekundären Kreislauf hervorgerufenen Wirkungen auf die Bruttoeinkommen der Primärverteilung berücksichtigt werden, da diese für den Erklärungs prozeß und die endgültigen Verteilungswirkungen von Bedeutung sind. Anhand 
exemplarischer Zinssteuerzahler-Zinsempfänger-Konstellationen sollen die strukturellen Verteilungswirkungen aufgezeigt werden.

b) Ausgangsmodell

Dem allgemeinen Demonstrationsziel entsprechend sollen die Ausgangskonstellationen möglichst einfach gehalten werden, um so die Wirkungsabläufe deutlicher verfolgen zu können, wobei auf die schon geschilderten Modellcharakteristika des kreislauftheoretischen Ansatzes zurückgegriffen wird.

Bei der Darstellung der. Verteilungswirkungen der Schuldaufnahme im KaldorModell wurde auf dessen Vorzug verwiesen, die institutionelle Verteilung erklären zu können. Diese wird auch in der vorliegenden Fragestellung angesprochen, da beide Gruppen zusätzlich staatliche Zinszahlungen und damit eine von den anderen Einkommen unterschiedliche Einkommensart beziehen können[172]. Entscheidend für den diesem Modell inhärenten Mechanismus ist die Unterstellung, daß die beiden Gruppen durch an realen Größen orientierte Verhaltensfunktionen zu kennzeichnen sind, die für alle von ihnen bezogenen Einkommensarten Gültigkeit haben. Wie für die Sparfunktionen soll auch für die hier verwendeten Steuerfunktionen lineare Homogenität unterstellt werden[173], wobei hervorzuheben ist, daß in unserem System der Staat lediglich durch diese (darüber hinaus) zweckbestimmte Steuererhebung und die Auszahlung der Beträge in Gestalt von Zinsen tätig wird.

Die Höhe der Zinszahlungen wird als exogen gegeben angenommen, was bei isolierter Betrachtung einer einzelnen Periode ein legitimes Vorgehen sein dürfte. Zunächst seien die verwendeten Symbole erläutert:

$\begin{array}{ll}\mathrm{Y} & =\text { Nettosozialprodukt } \\ \mathrm{I} & =\text { Nettoinvestition } \\ \mathrm{S} & \text { Gesamtersparnis } \\ \mathrm{Y}_{\mathrm{u}} & \text { Einkommen der Unternehmer (vor Besteuerung) } \\ \mathrm{Y}_{\mathrm{nu}} & =\text { Einkommen der Arbeitnehmer (vor Besteuerung) } \\ \mathrm{Y}_{\mathrm{u}}^{\mathrm{v}} & =\text { verfügbares Einkommen der Unternehmer } \\ \mathrm{Y}_{\mathrm{nu}} & =\text { verfügbares Einkommen der Arbeitnehmer } \\ \mathrm{Z}_{\mathrm{nu}} / \mathrm{Z}_{\mathrm{u}} & =\text { staatliche Zinszahlungen an Arbeitnehmer/Unternehmer } \\ \mathrm{C}_{\mathrm{nu}} / \mathrm{C}_{\mathrm{u}} & =\text { Konsum der Arbeitnehmer/Unternehmer } \\ \mathrm{S}_{n \mathrm{nu}} / \mathrm{S}_{\mathrm{u}} & =\text { Ersparnisbildung der Arbeitnehmer/Unternehmer } \\ \mathrm{T}_{n \mathrm{nu}} & =\text { Steuereinnahmen aus Besteuerung der Arbeitnehmer } \\ \mathrm{T}_{\mathrm{u}} & =\text { Steuereinnahmen aus Besteuerung der Unternehmer }\end{array}$




$$
\begin{array}{lll}
\mathrm{E}_{\mathrm{st}} / \mathrm{A}_{\mathrm{st}} & = & \text { Einnahmen/Ausgaben des Staates } \\
\mathrm{t}_{\mathrm{nu}} / \mathrm{t}_{\mathrm{u}} & = & \begin{array}{l}
\text { durchschnittliche (=marginale) Steuersätze für } \\
\text { Arbeitnehmer/Unternehmer }
\end{array} \\
\mathrm{s}_{\mathrm{nu}} / \mathrm{s}_{\mathrm{u}} & =\begin{array}{l}
\text { durchschnittliche (=marginale) Sparneigung der } \\
\text { Arbeitnehmer/Unternehmer }
\end{array}
\end{array}
$$

Obigen Überlegungen entsprechend gehen wir von einer Primärverteilung aus, die die Auswirkungen der staatlichen Kreditnahme der Vorperiode auf die Verteilungssituation dieser Periode umfaßt. Dieses Argument klingt zwar recht formal, da sich bei Annahme einer einmaligen Verschuldung keine materiellen Auswirkungen mehr zeigen, scheint aber aus methodischen Überlegungen an dieser Stelle wiederholenswert. Im folgenden sollen also

\begin{tabular}{|c|c|c|c|}
\hline IV . 1. & Y & $=$ & $Y_{u}+Y_{n u}$ \\
\hline IV. 2. & $\mathbf{Y}$ & $=$ & $\mathrm{C}_{\mathrm{u}}+\mathrm{C}_{\mathrm{nu}}+\mathrm{I}+\mathrm{A}_{\mathrm{st}}$ \\
\hline IV. 3. & $Y_{n u}^{v}$ & $=$ & $\mathrm{Y}_{n u}+\mathrm{Z}_{n u}-\mathrm{T}_{n u}$ \\
\hline IV . 4. & $\mathrm{Y}_{\mathrm{u}}^{\mathrm{v}}$ & $=$ & $Y_{u}+Z_{u}-T_{u}$ \\
\hline IV . 5. & $\mathrm{Y}_{\mathrm{nu}}^{\mathrm{V}}$ & $=$ & $C_{n u}+S_{n u}$ \\
\hline IV. 6. & $\mathrm{Y}_{\mathrm{u}}^{\mathrm{v}}$ & $=$ & $\mathrm{C}_{\mathrm{u}}+\mathrm{S}_{\mathrm{u}}$ \\
\hline IV. 7. & $\mathbf{S}$ & $=$ & $s_{n u}+s_{u}$ \\
\hline IV .8. & $\mathrm{~T}$ & $=$ & $\mathrm{T}_{\mathrm{nu}}+\mathrm{T}_{\mathrm{u}}$ \\
\hline IV. 9. & $\mathrm{Z}_{\text {st }}$ & $=$ & $\mathrm{Z}_{\mathrm{nu}}+\mathrm{Z}_{\mathrm{u}}$ \\
\hline IV. 10 & $\mathrm{Z}_{\text {st }}$ & $=$ & $\mathrm{T}$ \\
\hline IV . 11 & $T_{n u}$ & $=$ & $t_{n u} Y_{n u}$ \\
\hline IV. 12 & $\mathrm{~T}_{\mathrm{u}}$ & $=$ & $\mathrm{t}_{\mathrm{u}} \mathrm{Y}_{\mathrm{u}}$ \\
\hline IV. 13 & $\mathrm{~s}_{\mathrm{nu}}$ & $=$ & $s_{n u} Y_{n u}^{v}$ \\
\hline IV. 14 & $\mathrm{~S}_{\mathrm{u}}$ & $=$ & $\mathrm{s}_{\mathrm{u}} \mathrm{Y}_{\mathrm{u}}^{\mathrm{v}}$ \\
\hline
\end{tabular}
ausschließlich die Effekte im Vordergrund stehen, die von den staatlichen Zinszahlungen auf die Einkommensverteilung ausgehen. Das Modell setzt sich aus folgendem Gleichungssystem zusammen:

Gleichgewichtsbedingung

IV.15. I = $\mathrm{S}$ 
Bei den Gleichungen IV.1. - IV.6. handelt es sich um die schon bekannten definitorischen Ausgangsgleichungen. Die wieder stark vereinfachte Staatstätigkeit kommt in den Gleichungen IV.8. - IV.12. zum Ausdruck. Das gesamte Budget erschöpft sich in Zinszahlungen, wobei die Einnahmen über eine nicht weiter nach Einkommensarten differenzierende Proportionalsteuer auf die Gesamteinkommen der beiden Gruppen erzielt werden. Auf die Bedeutung der hier angenommenen Differenzierung der Sparneigungen wurde schon verwiesen. Für den vorliegenden Fall dürfte es eine plausible Annahme sein, daß sich die Verwendungsentscheidung bezüglich der staatlichen Zinszahlungen nicht von der der dominierenden Einkommensart der jeweiligen Gruppe unterscheidet.

Da die Parameter der Verhaltensgleichungen, also die Sparneigungen und die Steuersätze, als exogen gegeben angenommen werden, zudem durch Verzicht auf eine Investitionsfunktion gleiches für die Investitionen gilt, das reale Sozialprodukt durch die Vollbeschäftigungsannahme bei kurzfristiger Betrachtung und die Staatsausgaben durch Beschränkung auf die vorgegebenen Zinszahlungen determiniert sind, ist das wieder in Realgrößen definierte System geschlossen.

Es sollen im Rahmen dieses Gerüstes die Auswirkungen aufgezeigt werden, die von unterschiedlichen Besitzverhältnissen an Staatspapieren auf der Empfänger- und Steuerbelastungen auf der Aufbringerseite ausgehen werden. Für die Gültigkeit der Ergebnisse wäre es wünschenswert, wenn diese in eine möglichst allgemeine Form übergeführt werden könnten. Ausgangspunkt sollen zunächst zwei äußerst einseitige Situationen sein, die sich allerdings durch besondere Anschaulichkeit zu Demonstrationszwecken eignen. Zudem erleichtert eine solche Argumentation mit extremen (und dadurch wieder einfachen) Annahmen die Interpretation der Wirkungsabläufe. Es handelt sich dabei um die Fälle, in denen jeweils eine der verteilungsrelevanten Gruppen ausschließlich zur Finanzierung des Zinsendienstes herangezogen wird, während die andere ausschließlich Empfänger der Zinszahlungen ist. Wir unterstellen also zunächst eine völlig asymmetrische Verteilung der Staatspapiere. Das Vorgehen wird dabei immer zweistufig sein, da es einerseits die durch den staatlichen Eingriff bewirkte Entwicklung der Bruttoeinkommen und andererseits die der Nettogrößen, d.h. der verfügbaren Einkommen, zu beachten gilt. Letztlich ist zwar nur die Sekundärverteilung für die Verteilungssitutation des Individuums (bzw. der Gruppe) von Belang, doch ist zu ihrer Herleitung und Erklärung auch die Interpretation der Rückwirkungen auf die primären Einkommen vonnöten, da aufgrund der kreislaufbedingten Interdependenzen die Brutto-Faktoreinkommen immer auch von auf die Sekundärverteilung gerichteten Maßnahmen berührt werden.

Indikator für die Verteilungsrelationen sind folglich die Anteile des verfügbaren Unternehmer - bzw. Arbeitnehmereinkommens am Sozialprodukt, den jeweiligen Quoten der Faktoreinkommen kommt dagegen nur erläuternde Funktion für die Wirkungsabläufe zu. 
c) Verteilungswirkungen bei unterschiedlichen Aufbringer-Empfänger-Konstellationen

\section{$c_{1}$ ) Fall I Steuerzahler: Arbeitnehmer \\ Zinsempfänger: Unternehmer}

Im ersten Modellfall wird unterstellt, daß lediglich die Gewinnbezieher staatliche Papiere halten, folglich auch nur ihnen Zinszahlungen zufließen. Für die Finanzierung werden dagegen nur die Arbeitnehmer über eine (direkte) Proportionalsteuer zur Aufbringung herangezogen. Aus dem Gleichungssystem ergibt sich nach einigen Umformungen (zur genauen Herleitung vgl. Anhang I) folgender Ausdruck für die Bruttogewinnquote:

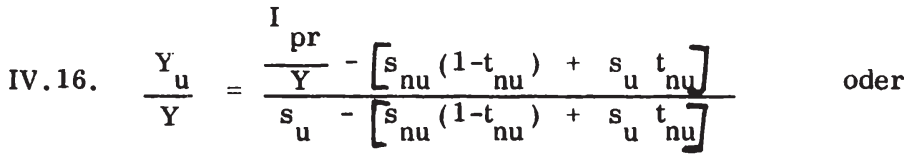

IV.17. $\frac{Y_{u}}{Y}=\frac{\frac{I_{p r}}{Y}-\left[s_{n u}+\left(s_{n}-s_{n u}\right) t_{n u}\right]}{s_{u}-\left[s_{n u}+\left(s_{n}-s_{n u}\right) t_{n u}\right]}$

Im Vergleich zu einer Situation ohne staatliche Tätigkeit, und auf diesen $\mathrm{Zu}$ stand werden wir uns immer beziehen[174], ist in Zähler und Nenner je ein zusätzlicher Summand zu beachten, der sich aus dem Produkt von Sparquotendifferenz und Steuersatz $t_{n u}$ ergibt. Aufgrund der Voraussetzung, daß die Sparneigung der Unternehmer über der der Arbeitnehmer liegt, ist dieser Term immer positiv kleiner eins. Da auch der Steuersatz als kleiner eins anzunehmen ist, wird der Summand die Stabilitätsbedingung $s_{u}>s_{n u}$ nicht verletzen können[175]. Als Ergebnis erhalten wir, daß sich der Anteil der Gewinneinkommen am Sozialprodukt verringert. Bei gegebenen Sparneigungen wird dieser Effekt um so stärker, je höher der Steuersatz (auf die Lohneinkommen) ist. Andererseits werden diese Verteilungsänderungen bei gegebenen Steuersätzen um so stärker, je größer die Differenz der Sparneigungen ist. Hier klingt der Zusammenhang zwischen Kaldorschem Sensitivitätskoeffizienten und Umfang der notwendig werdenden Verteilungsänderungen an.

Da Gleichung IV.17. lediglich die Entwicklung der Bruttogewinne angibt, soll nun die eigentlich verteilungsrelevante Sekundärverteilung untersucht werden. Der definitorische Zusammenhang zwischen den beiden Verteilungsformen ist hier recht einfach:

IV.18. $\frac{Y_{u}^{v}}{Y}=\frac{Y_{u}}{Y}+\frac{Z_{u}}{Y}=\frac{Y u}{Y}+\frac{t_{n u}\left(Y-Y_{u}\right)}{Y}=\frac{Y u}{Y}\left(1-t_{n u}\right)+t_{n u}$ 
Daraus ergibt sich (vgl. Anhang):

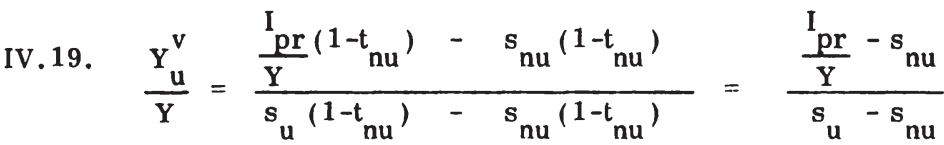

Als zunächst wohl überraschendes Ergebnis kann man festhalten, daß die tatsächliche Verteilungssituation der Gewinnbezieher nach Einbeziehung der Zinszahlungen im Vergleich zur Ausgangssituation völlig unverändert geblieben ist. Hervorzuheben ist dabei, daß von einer völlig asymmetrischen Empfänger/Aufbringer-Relation ausgegangen wurde, da ja eine Verteilungsgruppe nur Zinssteuern zahlen mußte.

Wie ist aber dieses Verteilungsergebnis zu interpretieren? Durch die Steuererhebung wird zunächst das verfügbare Nominaleinkommen der Arbeitnehmer und damit nach Maßgabe ihrer marginalen Konsumneigung auch deren nominale Konsumgüternachfrage reduziert. Da diese Mittel nun der Gruppe mit der höheren Sparneigung, den Unternehmerhaushalten also, zufließen, verringert sich dadurch die gesamtwirtschaftliche Nachfrage, die Unternehmergewinne aus dem Kreislauf werden zurückgehen. Wir befinden uns in einer Situation des Ungleichgewichts, da die freiwillige Ersparnisbildung die gütermäßig notwendige übersteigt[176]. Daran ändert auch das zunächst wegen der Zinszahlungen gestiegene Gesamteinkommen der Unternehmer nichts, da diese zwar absolut mehr konsumieren, diese Zunahme aber nicht den gleichzeitigen Entzugseffekt der Besteuerung der Arbeitnehmerhaushalte ausgleichen kann. Die auf diesen kontraktiven Impuls hin einsetzenden Preissenkungen[177] der Unternehmer auf der Anbieterseite bedeuten eine reale Einkommenserhöhung für die Arbeitnehmer, die bei annahmegemäß konstanter, an Realgrößen orientierter Konsumneigung zu einem weiteren Rückgang der nominalen Nachfrage und damit der Kreislaufprofite führt. Gleiches gilt für die Unternehmerhaushalte, deren nominale Einkommen durch tendenziell sinkende Kreislaufgewinne zurückgehen, so daß auch ihre nominale Konsumgüternachfrage sinken wird. Aber auch bei den Unternehmen als Nachfragern nach Investitionsguitern stellen sich diese Effekte ein. So werden bei real konstant bleibender Investitionsquote die nominalen Investitionsausgaben zurückgehen. Dieser Prozeß ist erst dann abgeschlossen, wenn die freiwilligen realen Ersparnisse der Unternehmer und der Arbeitnehmer bei gegebenen Verhaltensparametern in ihrer Summe der gesamtwirtschaftlichen Erfordernis entsprechen, d.h. die alten Verteilungsverhältnisse wiederhergestellt sind[178].

Durch die Einbeziehung der Zinszahlungsströme als Einkommensbestandteil der Gewinnbezieher werden die für diese Gruppe zuvor festgestellten negativen Verteilungswirkungen auf die Bruttogewinnquote, die sich als Ergebnis jeglicher zusätzlicher gesamtwirtschaftlicher Ersparnis einstellen, wieder neutralisiert. 
Aus dieser in der Argumentation des Transfer-Ansatzes äußerst ungünstigen Konstellation ergeben sich also in kreislauftheoretischer Sicht keine negativen Einflüsse auf die Einkommensverteilung. Die Verteilungsquoten der Sekundärverteilung spiegeln das Verteilungsergebnis wider, das vor Berücksichtigung dieses sekundären Kreislaufes gegeben war. Es sei hier auf den Anhang I verwiesen, der entsprechende Darstellungen für die Lohnquoten enthält. Diese Ableitungen weisen die komplementären Ergebnisse auf, die Quote der Bruttolohneinkommen nimmt zu, diese Entwicklung wird aber nach Berücksichtigung der Steuererhebung wieder egalisiert.

Entsprechend der umfassenden Stabilitätsbedingung ist in Analogie zu Gleichung III.16. für vorliegenden Fall folgende Ungleichung maßgebend[179]:

\section{$I / Y>s_{n u}+\left(s_{u}-s_{n u}\right) t_{n u}$}

Unterstellt man hypothetisch einen Steuersatz von eins $\left(t_{n u}=1\right)$, so wäre diese Relation nicht mehr erfültt, da sich auf der rechten Seite $s_{u}$ ergäbe, was voraussetzungsgemäß größer als die Investitionsquote sein muß. Von dieser Seite sind also Beschränkungen für die Wahl der möglichen Steuersätze zu erwarten, die in folgender Skizze dargestellt sind[180]:

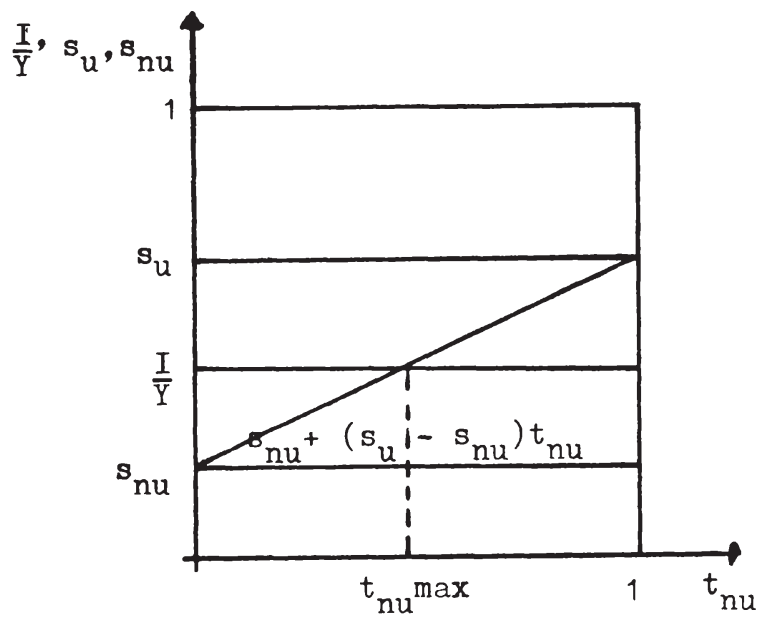

Auf der Ordinate werden die Sparneigungen der beiden Gruppen sowie die Investitionsquote abgetragen. Der Schnittpunkt der Funktion $s_{n u}+\left(s_{u}-s_{n u}\right) t_{n u}$, deren Steigung durch die Sparneigungen determiniert ist, mit der die Investitionsquote beschreibenden Geraden gibt den maximalen Steuersatz an, für den die Stabilitätsbedingung noch erfültt ist. Der Bereich der möglichen Steuersätze wird determiniert durch das Verhältnis der Investitionsquote zu den Sparneigungen. Je näher die Investitionsquote an der Obergrenze des Gleichgewichtsbereichs liegt, desto größer ist die Wahlmöglichkeit der Steu- 
ersätze, wobel diese Aussage unabhängig von den jewelligen Sparquotendifferenzen gilt.

Umgekehrt läßt sich festhalten, daß bei gegebenem I/Y und $s_{u}$ die Steuersätze um so höher llegen können, je geringer $S_{n u}$ ist. Geht man aber von einigermaßen realistischen Größen aus, zeigt sich, daß Steuersätze eines praktikablen Ausmaßes mit der Stabilitätsbedingung vereinbar sein dürften.

\section{$c_{2}$ ) Fall II Steuerzahler: Unternehmer Zinsempfänger: Arbeitnehmer}

Im zweiten Modellbeispiel sei die andere Extremalsituation unterstellt. Nun befinden sich alle staatlichen Papiere in Händen der Arbeitnehmer, wohingegen ausschließlich die Unternehmer über eine Proportionalsteuer zur Finanzierung des Zinsendienstes herangezogen werden. Bei analogem Vorgehen zu Fall I ergibt sich für die Quote der Bruttogewinneinkommen (zur Ableitung vgl. Anhang II):

IV .20

$$
\frac{Y_{u}}{Y}=\frac{\frac{I}{Y}-s_{n u}}{s_{u}\left(1-t_{u}\right)-s_{n u}\left(1-t_{u}\right)}=\frac{\frac{I}{Y}-s_{n u}}{\left[s_{u}-t_{u}\left(s_{u}-s_{n u}\right)\right]-s_{n u}}
$$

Auch hier ziehen wir als Beurteilungsmaß die Situation ohne staatliche Aktivi tät heran. Wir haben es mit schon bekannten Größen zu tun, da wiederum das Produkt aus Sparquotendifferenz und Steuersatz den Umfang der Änderung der Bruttoeinkommensverteilung determiniert. Allerdings nimmt hier die Gewinnquote $\mathrm{zu}$, da in Abhängigkeit von obigen Faktoren die Ersparnisbildung der Unternehmer reduziert wird. Gehen wir von der Gleichung IV.20, aus und ermitteln durch Berücksichtigung der Steuerabzüge das verfügbare Gewinneinkommen, so zeigt sich, daß dieses genau dem status quo entspricht:

IV.21. $\frac{Y_{u}^{v}}{Y}=\left(1-t_{u}\right) \frac{Y^{u}}{Y}=\frac{\frac{I}{Y}-s_{n u}}{s_{u}-s_{n u}}$

Es ergibt sich also das spiegelbildliche Ergebnis zum ersten Fall, auch hier bleiben die Verteilungs relationen unverändert. Es verändert sich zwar der Umfang der Bruttoeinkommen zugunsten der Gruppe der Gewinnbezieher, da die gesamtwirtschaftliche Ersparnis reduziert wird, doch wird dieser Effekt durch die Steuererhebung kompensiert. Entsprechend erhalten die Arbeitnehmer zusätzliche Einkommensbestandteile in Form der Zinszahlungen, doch wird dies durch die gleichzeitige Reduktion der realen Bruttoarbeitseinkommen konterkariert. 
Die Steuererhebung mindert zunächst das verfügbare Einkommen der Unternehmer, kehrt aber im Kreislauf in Abhängigkeit von der Konsumneigung der Arbeitnehmer wieder zurück, die nominale Gesamtnachfrage wird also erhöht. Damit ist wiederum eine Ungleichgewichtssituation gegeben, die nun aber auf einer im Vergleich zu den gütermäßigen Erfordernissen zu geringen gesamtwirtschaftlichen freiwilligen Ersparnisbildung beruht. Durch die im Umfang der Sparquotendifferenz ausgelösten Übernachfrage mit anschließender Preissteigerung, die bei den Unternehmern eine Erhöhung ihrer nominalen Investitions - und Konsumgüternachfrage notwendig macht, um diese Anteile real zu halten, ergeben sich die bekannten Umschichtungsprozesse, die erst dann auslaufen, wenn das reale Unternehmereinkommen eine Größe erreicht hat, die bei gegebenen Verhaltensparametern die alte Gleichgewichtssituation wieder herstellt.

Diese Verteilungssituation wird allerdings nur dann wieder eintreten, wenn im Verlauf des Prozesses keine neuen Ansprüche an das Sozialprodukt gestellt werden. Die reale Investitionsquote muß konstant bleiben, wobei dies bei insgesamt steigender nominaler Nachfrage nicht unbedingt angenommen werden kann, allerdings ist hier der restriktiv wirkende Einfluß der Besteuerung als Gegengewicht zu beachten. Daß die Konsumgüternachfrage der Unternehmerhaus halte auch bei steigenden Kreislaufgewinnen realiter nicht zunehmen wird, wurde schon oben bei Diskussion der Schuldaufnahme als plausible Hypothese anerkannt.

Nach Untersuchung dieser beiden Extremsituationen kann man zu dem vorläufigen Schluß gelangen, daß die Besitzstrukturen der Papiere und die Art der Zinssteuer für die institutionelle Verteilung ohne Belang zu sein scheint.

Auch für diesen zweiten Fall sei die oben verwandte Darstellung der Stabilitätsbedingung herangezogen, wobei nun die Ortslinie aller möglichen Steuersätze die Gerade

$$
\gamma=s_{u}-t_{u}\left(s_{u}-s_{n u}\right)
$$

darstellt[181]. Entscheidend für die realisierbaren Steuersätze ist das Gebiet links von ihrem Schnittpunkt mit der Geraden der Investitionsquote. Es gilt nun die umgekehrte Beziehung bezüglich der Wahlmöglichkeiten der Steuersätze und der Höhe der Investitionsquote. Je näher diese nämlich der unteren Grenze des Stabilitätsbereichs kommt, desto höher können die Steuersätze sein, ohne die Stabilitätsbedingung zu verletzen. 


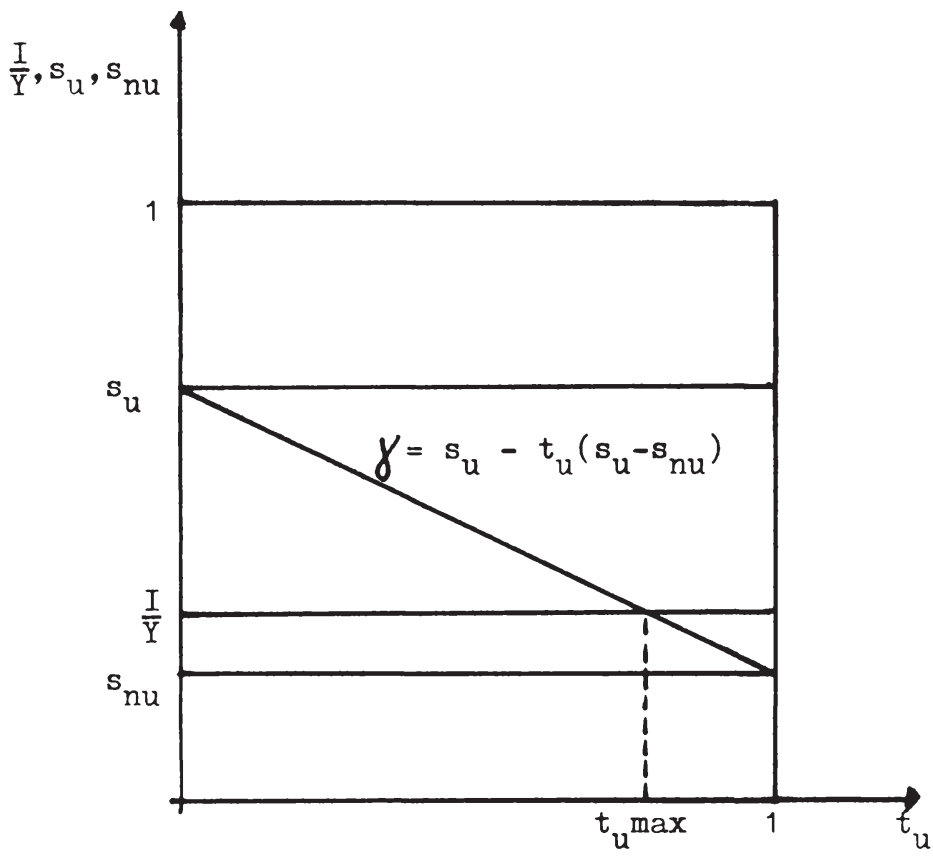

Wie sich aus dem Schaubild ergibt, dürften bei realistischen Annahmen bezüglich der Parameter auch hier keine Beschränkungen zu erwarten sein, dies könnte erst im Falle konfiskatorischer Steuersätze eintreten.

\section{$c_{3}$ ) Fall III Verallgemeinerung}

Im Gegensatz zu den vorhergehenden Fällen soll nun beiden Gruppen sowohl Empfänger- als auch Aufbringerfunktion zukommen. Dies sei mit folgenden Gleichungen ausgedrückt,

IV.22. $\mathrm{Z}_{\mathrm{u}}=\alpha \mathrm{T} ; \mathrm{Z}_{\mathrm{nu}}=(1-\alpha) \mathrm{T}$;

in denen der Verteilungsparameter $\alpha$ angibt, wie sich die staatlichen Papiere und damit die Zinszahlungen auf die beiden Gruppen verteilen. 
Aus unserem obigen Gleichungssystem ergibt sich für diesen gegenüber den bisherigen Zwischenschritten wohl als realistischer einzustufenden Fall folgender Ausdruck für die Bruttogewinnquote:

IV. 23.

$$
\frac{Y_{u}}{Y}=\frac{\frac{I}{Y}+\left[S_{n u}+\alpha\left(S_{u}-s_{n u}\right) t_{n u}\right]}{\left[S_{u}-(1-\alpha)\left(s_{u}-s_{n u}\right) t_{u}\right]+\left[s_{n u} \alpha+\left(S_{u}-s_{n u}\right) t_{n u}\right]}
$$

Die strukturelle Übereinstimmung mit den Gewinnquoten der zuvor besprochenen Fälle ist offenkundig. Zusätzlich kommt der Vertellungsparameter $\alpha$ ins Spiel, der zusammen mit dem Verhältnis der Steuersätze für die Gewichtung der ansonsten schon oben beschriebenen Einflußfaktoren maßgeblich ist.

Diese wirken dabei gegenläufig, da der positive Summand (in Zähler und Nenner auftretend) eine Reduzierung, der zweite Term im Nenner aber eine Erhöhung dieser Gewinnquote bewirkt.

A priori läßt sich keine eindeutige Aussage über die Veränderungsrichtung treffen, die abhängig vom quantitativen Einfluß der beiden Gegenkräfte ist. Für den Einfluß der Steuersätze auf die Verteilung können wir auf die oben gewonnenen Ergebnisse verweisen, wonach die jeweilige Verteilungsänderung (i.e. positiv bei $t_{u}$ und negativ bei $t_{n u}$ ) mit der Höhe der Steuersätze korreliert. Da die beiden Steuersätze bei gegebenen Zinsverpflichtungen voneinander abhängig sind, ist keine eindeutige Aussage möglich, da die gegenläufigen Effekte saldiert werden müssen. Gleiches läßt sich für die Verteilungsparameter festhalten, da sich auch diese beiden Größen zu eins ergänzen.

Gibt man die Voraussetzung eines gegebenen Zinsniveaus auf, so lassen sich zwar für die Steuersätze, nicht aber für die Verteilungsparameter eindeutige Ergebnisse ableiten. Müssen nämlich die Steuersätze bzw. ein Steuersatz aufgrund gestiegener staatlicher Zinszahlungs verpflichtungen erhöht werden, so läßt sich innerhalb der Stabilitätsbedingungen festhalten, daß eine Lohnsteuererhöhung ein Sinken obiger Gewinnquote, ein Erhöhen der Gewinnsteuer aber ein Steigen derselben mit sich bringt.

Die anderen Verhaltensgrößen spielen an dieser Stelle keine entscheidende Rolle, da unmittelbar ersichtliche Kausalzusammenhänge zwischen ihnen und den hier diskutierten Problemen nicht bestehen.

Die Diskussion der Verteilung der Bruttoeinkommen soll aber nicht zu weit getrieben werden, da diesem Ausdruck, wie oben gezeigt wurde, nur mittelbare verteilungspolitische Bedeutung zukommt. Es soll deshalb wieder die Verteilung der verfügbaren Einkommen bestimmt werden, die die Zinszahlungen an die beiden Gruppen mitberücksichtigt. Der definitorische Zusam- 
menhang ist hier allerdings komplexer:

IV 24. $\frac{Y_{u}^{v}}{Y}=\left(1-t_{u}\right) \frac{Y_{u}}{Y}+Z_{u}=\left(1-t_{u}\right) \frac{Y_{u}}{Y}+\alpha t_{n u} \cdot \frac{\left(Y-Y_{u}\right)}{Y}$
$+\alpha t_{u} \cdot \frac{Y_{u}}{Y}$

Die Umformungen sind im Anhang ( III ) nachvollziehbar, als Ergebnis erhält man:

IV. 25. $\frac{Y_{u}^{v}}{Y}=\frac{\frac{I}{Y}-s_{n u}}{s_{u}-s_{n u}}$

Auch bei Berücksichtigung gemischter Aufbringungs - und Empfängerstrukturen ergibt sich das für die isolierten Fälle erzielte Ergebnis, daß nämlich die institutionelle Verteilung durch den sekundären staatlichen Kreislauf nicht verändert wird.

Diese absolute Aussage muß relativiert werden, da die Stabilitätsbedingungen noch nicht auf mögliche Beschränkungen der Parameter untersucht wurden. Auf den isolierten Stabilitätsbetrachtungen aufbauend sollen mögliche Restriktionen für den allgemeinen Fall untersucht werden. Die Stabilitätsbedingung lautet $s_{u}-(1-\alpha)\left(s_{u}-s_{n u}\right) t_{u}>I / Y>s_{n u}+\alpha\left(s_{u}-s_{n u}\right) t_{n u}$, d.h. in das schon bekannte Ungleichungssystem spielt zusätzlich der Strukturparameter $\alpha$ hinein. Folglich ergibt sich in der graphischen Darstellung des allgemeinen Modells für die beiden Funktionen jeweils eine Schar von Geraden, die allen möglichen Werten von $\alpha$ bzw. $(1-\alpha)$ entsprechen. Auf der rechten Ordinate wird $\alpha$ von unten nach oben aufgetragen, entsprechend ergibt sich der komplementäre Wert von $(1-\alpha)$ in umgekehrter Richtung. Da die Grenzfälle mit den oben geschilderten Beispielen zusammenfallen, ergibt sich insgesamt eine Erweiterung des Bereichs möglicher Steuersätze bei einer Verteilung der Staatspapiere (wie auch immer differenziert) iber beide Gruppen. Diese Aussage gilt unabhängig von der aktuellen Höhe der Investitionsquote. 


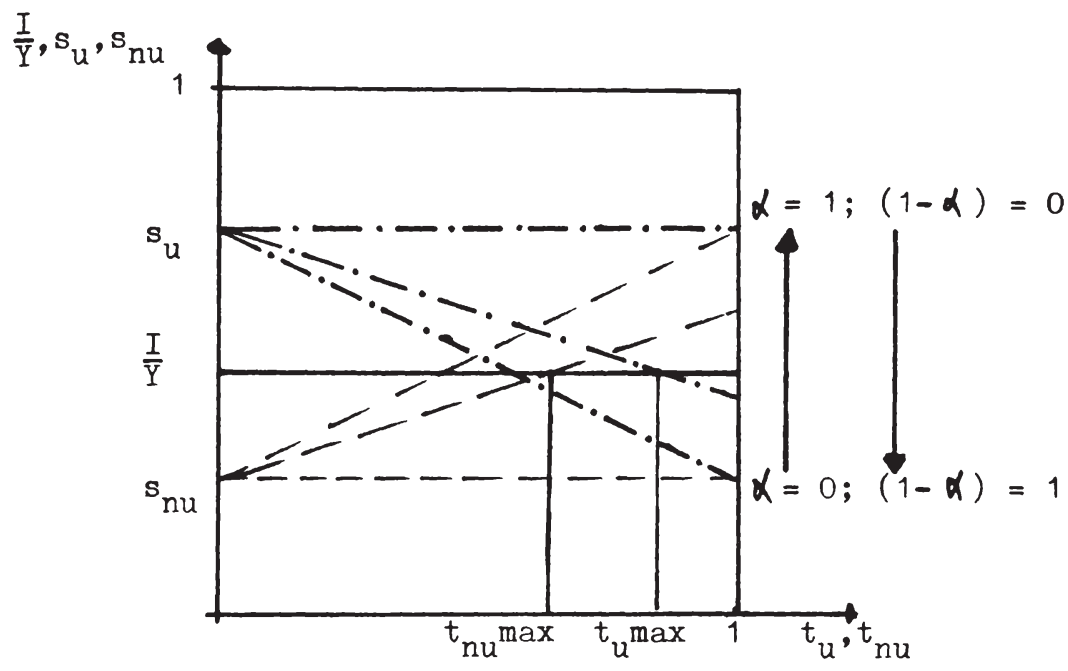

Stellt man die Frage nach möglichen Einschränkungen, können höchstens für den Fall einer völlig einseitigen Verteilung (zugunsten der Arbeitnehmer) für den Steuersatz $t_{n u}$ Restriktionen erwartet werden. Schon bei einer Gleichverteilung $\quad \alpha=(1-\alpha)=1 / 2$ könnte dieser Steuersatz aber beträchtliche Höhen erreíchen, die iber dem derzeitigen Spitzensteuersatz lägen. Insofern kann man davon ausgehen, daß bei nahezu beliebiger Wahl der Parameter ökonomisch sinnvolle Ergebnisse zu erwarten sind.

\section{d) Schlußfolgerungen}

Resümierend läßt sich festhalten, daß die von den Vertretern des Transferansatzes angenommenen Verteilungswirkungen - von der methodischen Problematik dieses Ansatzes ganz abgesehen - in kreislauftheoretischer Sicht nicht zutreffend sind. Für ein Zwei-Klassen-Schema ergibt sich, daß die Einkommensverteilung zwischen diesen beiden Gruppen durch den zusätzlichen Steuer-Zins-Kreislauf nicht verändert werden muß. Dieser Sachverhalt stellt ein plastisches Beispiel für die mögliche Diskrepanz zwischen formaler und effektiver Inzidenz dar.

Allerdings ist für dieses Ergebnis noch einmal die Bedeutung der Annahme eines rein an Realgrößen orientierten Nachfrageverhaltens der Wirtschafts subjekte hervorzuheben. Denn nur bei einem solchen Verhalten stellt sich das alte Verteilungsbild selbst dann wieder ein, wenn die Unternehmer zusätzliche, von den Arbeitnehmern aufgebrachte nominale Einkommensbeträge 
in Form der Zinszahlungen erhalten. In dem Maße, in dem die Unternehmerhaushalte einer Geldillusion unterlägen und sie ihre reale Konsumgüternachfrage steigerten, würden dann Verteilungsänderungen $\mathrm{zu}$ ihren Gunsten eintreten.

Die oben betrachteten Vorgänge des staatlichen Eingreifens stellen eine neue Querverteilung her, indem nominale Einkommensbeträge von bestimmten Haushalten erhoben und an andere weitergeleitet werden; führt man aber in einem solchen Zwei-Klassen-System - von einem beliebigen Gleichgewichtszustand ausgehend - eine Datenänderung ein, die eine neue Querverteilung mit sich bringt, so wird im neuen Gleichgewicht der reale Einkommensanteil der Unternehmer wieder so hoch sein, daß in einem der gesamtwirtschaftlichen Vermögensbildung entsprechenden Umfang freiwillig Ersparnis gebildet wird[182]. Die durch die Transferierung zwischen den Gruppen mit unterschiedlicher Sparneigung notwendig werdenden Veränderungen der Ersparnisbildung können ceteris paribus nur über Verteilungsumschichtungen erreicht werden, die sich durch entsprechende Variationen des Preisniveaus einspielen. Dabei bringt eine Verengung der Nachfrageströme (Fall I) deflatorische, eine Erweiterung der Nachfrageströme (Fall II) inflatorische Effekte mit sich. Deren Ausmaß bestimmt sich durch die Höhe der notwendig werdenden Veränderung der gesamtwirtschaftlichen Ersparnisbildung, die einerseits von der Differenz der Sparneigungen der beiden Gruppen und andererseits vom Umfang der Zinszahlungen (und damit der Steuersätze) abhängig ist.

Die Einfluisse des Steuer-Zins-Kreislaufs bleiben solange verteilungspolitisch irrelevant, wie die verteilungsdeterminierende Verwendungsstruktur konstant bleibt[183]. Diese Unterstellung dürfte bezüglich der Sparfunktionen recht plausibel sein, da nicht anzunehmen ist, daß sich diese aufgrund der staatlichen Zinszahlungen wesentlich ändern. Entscheidender Einfluß kommt dann dem Verhalten der privaten Investitionsgüternachfrage zu.

Standen bei Betrachtung der Schuldaufnahmephase eindeutig die Investitionsmöglichkeiten als möglicherweise restriktiv wirkender Faktor im Vordergrund, so wird im Falle der Sekundärverteilung auch unmittelbar die Reaktion der Investitionsneigung auf die Gewinnbesteuerung relevant[184]. Man könnte nun argumentieren, daß aufgrund der geringen Höhe der Steuersätze keine restriktiven Wirkungen zu erwarten sind, doch gilt dies nur in isolierter Sicht. Ginge man von einem erweiterten Modell mit voller Staatstätigkeit aus, so hätte die Behandlung der gesamten incentive-Problematik durchaus ihre Berechtigung. Gerade eine Erhöhung der Steuersätze, und dies wäre das realistische Pendant zu dem Fall der Einführung einer neuen Steuer, könnte bei Überschreiten bestimmter Marken Wirkungen auf das Investitionsverhalten mit sich bringen. Damit ist aber indirekt ausgedrückt, daß innerhalb eines weiten Bereichs keine ins Gewicht fallende Abhängigkeit gegeben sein dürfte[185]. Doch muß die Möglichkeit einer Reaktion der privaten Investitionsguiternachfrage auf eine Steuererhöhung eingeräumt werden[186], wenn sie in unserem abstrahierenden Modell und für die daraus abgeleiteten prinzipiellen Zusammenhänge auch keine Rolle spielen wird. 
Das Resultat, daß die Verteilung der Staatspapiere einerseits und der Aufbringungslasten andererseits keine distributionspolitisch relevanten Effekte mit sich bringen, muß angesichts des hohen Aggregationsgrades relativiert werden, da sich hinter dieser globalen Aussage durchaus verteilungsrelevante Vorgänge struktureller Art verbergen können. Es sei von dem Fall ausgegangen, daß die Beträge für die Zinszahlungen nur von der Gruppe der Lohnbezieher aufgebracht, aber auch nur von dieser Gruppe bezogen werden. Sollten sich daraus keine verteilungsrelevanten Effekte innerhalb der Gruppe ergeben, müßten alle pro Kopf oder in Relation $\mathrm{zu}$ ihrem Einkommen die gleichen Anteile aufbringen und erhalten.

Auf der anderen Seite werden sich im großen Aggregat der Unternehmer in der hier getroffenen Abgrenzung auch solche Selbständige verbergen, die keinen Anteil an Kreislaufprofiten haben werden. Ihre Aufnahme in dieses Aggregat drückt die Durchschnittswerte der Sparneigung, sie selbst werden aber nicht von den Veränderungen der Kreislaufgewinne betroffen, die aus Kontraktionen und Expansionen der gesamtwirtschaftlichen Nachfrage resultieren.

Dies stellt aber das grundsätzliche Problem einer solchen Aggregation dar, in der viele Einzelfälle mit von den makroökonomischen Ergebnissen abweichenden Resultaten zu finden sind. So ist daran zu erinnern, daß auch Abweichungen von der Zusammensetzung der Einkommen in ihren Auswirkungen auf die Sparneigung nicht erfaßt werden. Diese strukturellen Betrachtungen sollten lediglich die Grenzen der Aussagefähigkeit makroökonomischer Ableitungen andeuten; vor einer einfachen Übertragung dieses Resultats auf individuelle Verteilungssituationen ist also zu warnen. Die Aussagen bezüglich der effektiven Inzidenz der Zinssteuern beinhalten solche Effekte nicht. Dies muß als Manko aller entsprechend aggregierter Ansätze hingenommen werden, die Relativierung der Ergebnisse erscheint angebracht, wenn man etwa an die mögliche Übertragung dieser Resultate auf allgemeine Probleme staatlicher Transferzahlungen denkt.

Trotz obiger Einschränkung stellen aber diese Überlegungen, die zum Ergebnis führen, daß die Besitzverhältnisse an staatlichen Papieren und die Aufbringung der Steuern keine determinierende Rolle für die Verteilung spielen, einen wichtigen Schritt auf dem Weg zur Bestimmung der effektiven Inzidenz dar. Die verteilungspolitische Relevanz wird besonders deutlich, wenn man diese Ergebnisse mit denen des Transferansatzes, der über eine mikroökonomischer Partialanalyse verhaftete formale Inzidenzbetrachtung nicht hinausgeht, und den daraus abgeleiteten verteilungspolitischen Schlußfolgerungen vergleicht. Demgegenüber rückt die kreislauftheoretische Betrachtung auch die Vermögens anlage in staatliche Papiere in den Gesamtzusammenhang der Vermögensbildung. Die zuletzt angestellten Überlegungen zur effektiven Inzidenz der Zinssteuern stellen ja nur einen Teil der gesamten Verteilungswirkungen staatlicher Schuldaufnahme dar. 
Nach Ableitung dieser effektiven Inzidenz kann ein erster Überblick iber die in kreislauftheoretischer Sicht relevanten Effekte der staatlichen Verschuldung gegeben werden. Die entscheldenden Verteilungsänderungen spielen sich in der Periode der staatlichen Schuldaufnahme ab. Die Verteilungswirkungen zugunsten der Unternehmereinkommen infolge einer einmaligen Schuldaufnahme sind dabei von nur temporärer Natur, da sie in der nächsten Periode nicht mehr nachwirken. Da auch die in den Verzinsungsperioden erhobene Zinssteuer und deren Auszahlung keine entscheidenden Verteilungseffekte mit sich bringen wird, sind alle Verteilungseffekte auf die Phase der Schuldaufnahme konzentriert. Daß für diese Überlegungen nur innerhalb bestimmter Grenzen Gültigkeit beansprucht werden kann, ist selbstverständlich, da ab gewissen Höhen sowohl die Frage nach der Elastizität des Bankensystems als auch die nach der incentive-Problematik der Besteuerung nicht mehr eindeutig beantwortbar ist und die Möglichkeit der Rückwirkung des Verteilungs mechanis mus auf die Verhaltensparameter nicht von der Hand zu weisen ist. 

Fünftes Kapitel

INTERTEMPORALE VERTEILUNGSWIRKUNGEN

A) VORÜBERLEGUNGEN

1. Struktur - und Niveaueffekte

Das bisherige Untersuchungsziel war, die Verteilungseffekte interpersoneller Art aufzuzeigen, die mit einem durch Kreditaufnahme bewirkten Ressourcenübergang vom privaten in den öffentlichen Bereich verbunden sind. Da wir von einer Situation der Vollbeschäftigung ausgingen, war das Konkurrieren dieser beiden Bereiche um die vorgegebenen Ressourcen der Grundtatbestand, wobei sich je nach makroökonomischem Wirkungsablauf unterschiedliche strukturelle Verteilungseffekte für den privaten Sektor ergaben. Implizit wurden dabei insofern schon Verteilungseffekte intertemporaler Art erfaßt, als die Entwicklung von Primär-und Sekundärverteilung in der Schuldaufnahmephase und den folgenden Verzinsungsperioden, also die chronologische Entwicklung der strukturellen Verteilung, verfolgt wurde.

Bei diesem Vergleich der strukturellen Verteilung der Schuldaufnahmephase und der darauffolgenden Perioden ergab die kreislauftheoretische Betrachtung, daß die Verteilung in der Folgezeit wieder die Werte einnimmt, die sie vor der staatlichen Kreditnahme hatte[187]. Diese Aussage ist unabhängig davon, ob bzw. welche Veränderungen in der Periode der Schuldaufnahme eingetreten sind[188]. Es ist dabei allerdings an die Annahme zu erinnern, daß es sich um eine einmalige staatliche Schuldaufnahme handelte.

Da auch für die Verzinsungsphase die kreislauftheoretische Untersuchung des Steuer-Zins-Kreislaufs keine relevanten Verteilungseffekte ergab, kann man für die strukturelle Verteilung zum Schluß kommen, daß diese in den Folgeperioden den Wert einnimmt, den sie vor dem betrachteten staatlichen Impuls innehatte, die strukturellen Verteilungseffekte sich also ausschließlich in der Schuldaufnahmephase abspielen. Neben diesen strukturellen Effekten muß aber in intertemporaler Sicht die nicht minder bedeutsame Entwicklung der Verteilungsniveaus beachtet werden. Auch zur Beantwortung dieser Frage sind die sich einstellenden Crowding-out-Effekte der entscheidende Faktor, da sie darüber Auskunft geben, ob und in welchem Umfange die das gesamtwirtschaftliche Wachstum beeinflussenden privaten Investitionen tangiert werden[189].

Wie oben aufgezeigt wurde, entspricht das Eintreten solcher Effekte den Annahmen des klassischen Ansatzes, während im keynesianischen System aufgrund expansiver Nachfragetendenzen eher Stimuli für die privaten Investitionen zu erwarten waren. 
Vor dem Hintergrund der bisher abgeleiteten strukturellen Verteilungseffekte sollen nun einige der bekanntesten Ansätze zur Untersuchung der intertemporalen oder intergenerativen Effekte einer staatlichen Verschuldung auf ihre verteilungspolitische Bedeutung untersucht werden.

\section{Lastdebatte}

Die noch nicht beendete[190] Diskussion um diesen Aspekt der Staatsverschuldung ist unter dem Begriff der Lastdebatte bekannt, die die Frage zu klären sucht, ob eine staatliche Kreditaufnahme im Gegensatz zur Steuerfinanzierung dazu geeignet ist, intertemporale Äquivalenzvorstellungen bei der Finanzierung staatlicher Projekte zu realisieren. Unserem Inzidenzkonzept zufolge soll keine Antwort auf diese Fragestellung gegeben, sondern es sollen ausschließlich die intertemporalen und intergenerativen Verteilungseffekte der Staatsverschuldung aufgezeigt werden. Die Vorbehalte gegenüber diesem $\mathrm{Be}-$ griff der "Last" wurden schon angesprochen, nach Hedtkamp wird bei einer solchen Argumentation "das Bild vom Moloch Staat" lebendig[191]. Auch dies veranschaulicht die Bedeutung, die einer wertfreien und unmittelbar auf relevante ökonomische Sachverhalte abstellenden Terminologie zukommt, weswegen die in der Literatur zu finderiden Ansätze einer intertemporalen Betrachtung im Rahmen der bisherigen verteilungstheoretischen Analyse beurteilt werden sollen. Dies schließt allerdings nicht aus, daß wir uns bei der Darstellung der Standpunkte einzelner Autoren auch des von innen verwendeten Lastbegriffs bedienen.

Zunächst sei noch auf die Abgrenzung der intergenerativen und intertemporalen Effekte eingegangen. Wir verstehen unter intertemporalen Wirkungen einen Oberbegriff, der ganz allgemein die Auswirkungen einer in einer bestimmten Periode getroffenen Maßnahme auf (hier verteilungsrelevante) Größen in den Folgeperioden umfaßt. Es handelt sich dabei zunächst um ein allgemeines Phänomen, da wohl alle wirtschaftlichen Handlungen in einem zeitlichen Kontext gesehen werden müssen. Der intergenerative Aspekt unterscheidet sich dadurch vom intertemporalen, daß hier bestimmte Gruppen bzw. Individuen herausgegriffen werden. Die Diskussion leidet vielfach unter der sehr unterschiedlichen Abgrenzung des Generationenbegriffs[192], so daß wir zunächst nur von intertemporalen Effekten ausgehen wollen, im Einzelfall aber die Abgrenzung des jeweiligen Autors heranziehen.

\section{Organizistische versus individualistische staatsaufassung}

In anderer Formulierung läuft die zentrale Fragestellung der sogenannten Lastdebatte darauf hinaus, inwieweit die Wirkungen einer staatlichen Schuldaufnahme mit denen einer Kreditnahme durch private Wirtschaftssubjekte vergleichbar sind. Die einzelwirtschaftliche Verschuldung ermöglicht es dem Individuum, seine Ausgabenpläne im Zeitablauf variabler zu gestalten. Zu- 
sätzliche monetäre Mittel beinhalten auch die Möglichkeit, zusätzliche Anspruiche an das Sozialprodukt durchzusetzen. Dem steht das Versprechen gegenüber, in einer späteren Periode diese Mittel an den Gläubiger zurückzuzahlen, was einen in der Zukunft liegenden Verzicht auf Einkommensbestandteile bedeutet. Auch im Zusammenhang mit den intertemporalen Effekten einer öffentlichen Verschuldung bedienen sich einige Autoren dieser Argumentation. Der Staat als haushaltspolitischer Entscheidungsträger kann durch Kreditfinanzierung erreichen, daß für die Wirtschaftssubjekte in dieser Periode kein Einkommensverzicht notwendig wird, dieser muß erst in der Folgezeit für Verzinsung und Tilgung geleistet werden[193].

Vertretern eines solch utilitaristisch-individualistischen Standpunktes, für die stellvertretend Buchanan genannt werden kann, stehen Autoren einer organizistisch ausgerichteten Staatsauffassung wie Lerner gegenüber[194], die für die Gesellschaft als Ganzes keine solche Vorgriffsmöglichkeiten als gegeben erachten, da die Ressourcen in jedem Falle zum Zeitpunkt der Schuldaufnahme[195] vom Staat beansprucht werden. Eine Verschuldung des öffentlichen Sektors kann in einer Vollbeschäftigungssituation rein gütermäßig nicht auf ein höheres Niveau führen. Die Gesellschaft als Ganzes betrachtet (und insofern handelt es sich durchaus um eine organizistisch-kollektivistische Sicht) kann nur in dem Umfange Güter beanspruchen, den das Produktionspotential erlaubt[196]. Intertemporale Effekte in dieser organistischen Sicht können sich aber daraus ergeben, daß durch die Finanzierungsart Einfluß auf die Entwicklung der gesamtwirtschaftlichen Verteilungsniveaus, d.h. Einfluß auf die Wachstumsraten genommen wird.

Es soll hier nicht auf normative Fragestellungen der Wachstumspolitik eingegangen, sondern nur die Faktoren aufgezeigt werden, die den Zusammenhang zwischen allokativer Entscheidung in der Verschuldungsphase und der Entwicklung der Verteilungsniveaus in den Folgeperioden bestimmen. Nach der Auseinandersetzung mit diesen auf die Dichotomie zwischen privatem und öffentlichem Sektor abstellenden makroökonomischen Ansätzen sollen die auch ordnungspolitisch begründeten individualistischen Ansätze dargestellt werden. Wir werden mit Ricardo beginnen, auf den sich alle mikroökonomischen Ansätze zurückführen lassen, um dann zu Buchanan als dem zeitgenössischen Hauptvertreter dieser Richtung zu kommen. Seine zahlreichen Veröffentlichungen haben diese Kontroverse neu belebt. So sind auch die auf intergenerative Effekte abstellenden Überlegungen von Bowen-Davis-Kopf und Head als Ergänzung oder Kritik an Buchanan zu verstehen.

Diese mikroökonomischen Ansätze stellen auf intertemporale Effekte für das Individuum $a b$, so daß es besonders reizvoll scheint, diese (differierenden) Ergebnisse mit denen der organizistischen Sicht zu vergleichen. Primäres Ziel wird dabei sein, die jeweiligen Aussagen auch in verteilungspolitischer Terminologie zu erfassen, um so eine Vergleichbarkeit der Inhalte zu erreichen und die Ursachen für verbleibende Divergenzen eindeutig $\mathrm{zu}$ isolieren. 


\section{B) MAKROÖKONOMISCHER ANSATZ}

\section{Darstellung des Wachstumsansatzes}

Ausgangspunkt makroökonomischer Betrachtungen zu intertemporalen Verteilungseffekten einer staatlichen Schuldaufnahme ist der simple Tatbestand, daß die vom Staat beanspruchten Ressourcen in jedem Falle in der Schuldaufnahmephase privater Verwendung entzogen werden[197]. Dies stellt die zentrale Aussage der Vertreter der sogenannten Neuen Orthodoxie dar[198], die daran die Folgerungen knüpfen, daß keine Belastung zukünftiger Perioden möglich ist. Anfang der sechziger Jahre[199] wurde dieser Ansatz von verschiedenen Autoren dahingehend modifiziert[200], daß man auf die Effekte der staatlichen Schuldaufnahme abstellte, die sich durch die Beeinflussung der gesamtwirtschaftlichen Allokation in der Verschuldungsphase ergaben. Dieser Aggregate Investment Approach oder Wachstumsansatz hat die Entwicklung der für das Wachstum und damit die Einkommensströme zukünftiger Perioden relevanten Aggregate, nämlich der gesamtwirtschaftlichen Investitionen, im Auge. Es sind also die Auswirkungen bestimmter Finanzierungsmaßnahmen auf die gesamtwirtschaftliche Wachstumsrate determinierend für ihre intertemporalen Verteilungseffekte. Damit wird ein und derselbe Ursachenkomplex sowohl für die interpersonellen als auch intertemporalen Verteilungseffekte entscheidend.

Im Zuge diese ${ }_{1}$ Lastdebatte werden meist dem Differentialinzidenzkonzept entsprechend die finanzierungsspezifischen Auswirkungen von Staatsverschuldung und Steuern auf Konsum und Investition verglichen[201]. Eine solche Betrachtung ist aber auch allein für die staatliche Schuldaufnahme von Bedeutung, da nicht von vornherein bestimmte Wirkungsabläufe mit ihr verbunden sein müssen. Wenden wir diesen Ansatz auf die staatliche Neuverschuldung an, so ist damit wieder der Umfang des Crowding-out angesprochen.

Zur Messung oder approximativen Quantifizierung der auftretenden Wachstumsverluste müßte die Produktivität und der Umfang der nicht zum Zuge gekommenen Privatinvestitionen bekannt sein[202]. Es muß aber bedacht werden, daß auch die so finanzierten staatlichen Ausgaben einen Wachstumsbeitrag liefern, der kleiner, aber auch größer oder gleich dem der nicht realisierten Privatinvestitionen sein kann. Die oben aufgezeigte Bruttobelastung wäre durch Berücksichtigung der Wachstumseffekte der staatlichen Ausgaben zu einer Nettogröße zu relativieren. Die saldierende Sicht könnte also auch einen positiven Nettobeitrag des staatlichen Eingreifens ergeben. Nun entspricht die Berücksichtigung von Produktivitätseffekten staatlicher Ausgaben nicht mehr unserem Inzidenzkonzept, das von der Leistungsseite des staatlichen Ausgabenverhaltens abstrahiert. Dies stellt für die Aussagefähigkeit unseres Konzepts kein Manko dar, da es hier nur um intertemporale Effekte geht, d.h. um die Frage des zeitlichen Auftretens bestimmter Auswirkungen. Sind die staatlichen Ausgaben im obigen Sinne "unproduktiv", so ist dies zunächst einmal eine Folge der staatlichen Ausgabenentscheidung. In jedem Falle erfährt dann die Volkswirt- 
schaft eine nicht optimale Ressourcenallokation, für den Zeitpunkt des Auftretens solcher Effekte kann allerdings die Finanzierungsart entscheidend sein [203].

Unabhängig von der Produktivität staatlicher Ausgaben läßt sich die tendenzielle Aussage festhalten, daß die Finanzierungsart öffentlicher Ausgaben dann einen Einfluß auf das Wachstum und damit auf künftige Verteilungspotentiale nehmen wird, wenn durch sie die privaten Investitionen tangiert werden, da andernfalls die Wachstumseffekte noch stärker wären[204]. Nun stellt die Produktivität und damit die wachstumspolitische Bedeutung staatlicher Ausgaben ein vieldiskutiertes Feld dar, in dem generelle Vorbehalte gegenüber staatlichen Projekten ebenso wie ein generelles Attestieren von wachstumspolitischer Bedeutung in Form nicht näher definierter Umwegproduktivität zu finden sind. Es ist also ein schwieriges, kasuistisches Unterfangen, zu Bewertungen zu gelangen. Obige nur relative Aussagen haben den Vorzug, daß wir uns mit dem Problem der Größe und der Bewertung der auf die künftigen Perioden verschobenen "Lasten", denn nichts anderes bedeuten ja die verschiedenen realisierten bzw. nicht realisierten Verteilungspotentiale, nicht auseinanderzusetzen haben. Hierzu wäre eine intertemporale normative Vorgabe als Maßstabsgröße notwendig. Schließlich könnte im Extremfall jede nicht unternommene Investition als eine Belastung künftiger Perioden interpretiert werden, sieht man von der Wahrung eines sozialkulturellen Substistenzminimums für die gesamte Volkswirtschaft ab[205].

Es sei nochmals betont, daß unter der Prämisse einer gegebenen Ausgabenentscheidung argumentiert wurde. Stellt man diese nämlich zur Diskussion, so müßten unter wachstumspolitischen Gesichtspunkten tatsächlich die Wachstumseffekte der geplanten Staatsausgaben gegen die der möglicherweise verdrängten Privatinvestitionen aufgerechnet werden.

\section{Verteilungspolitische Beurteilung des Wachstumsansatzes}

Der Aggregate Investment Approach stellt seiner gesamtgesellschaftlichen Ausrichtung gemäß die Entwicklung der Verteilungsniveaus in den Mittelpunkt, die den Verteilungspolitiker üblicherweise stärker interessierenden Fragen der Entwicklung der Verteilungssituation bestimmter Gruppen läßt er dagegen of fen. Charakteristisch hierfür ist auch die Einschätzung der Zinszahlungen des Staates, die deshalb kein Verteilungsproblem darstellen (bzw. in der Terminologie dieses Ansatzes keine Last), weil lediglich Transfers von der linken in die rechte Tasche fließen, für die Gesellschaft insgesamt also keine Belastung entsteht (we-owe-it-to-ourselves)[206].

Die Determinanten für die Entwicklung der Verteilungsniveaus sind dabei identisch mit den Faktoren, die auch in kreislauftheoretischer Sicht für die strukturellen Verteilungswirkungen einer staatlichen Schuldaufnahme maßgeblich waren. Es stellt sich nun ein konträres Verhältnis dar. Ergab sich nämlich bei einer völligen Verdrängung privater Investitionen in der Aufnahmephase keine 
Auswirkung auf die strukturelle Verteilung, so stellen sich dann die stärksten Effekte auf das Verteilungspotential der folgenden Perioden ein. Setzen sich dagegen die Ansprüche des Staates in voller Höhe zusätzlich zu den privaten Investitionen durch, so bleibt der Expansionspfad der Verteilungs niveaus unberührt (bel unterstellter Unproduktivität der Staatsausgaben) oder steigt sogar an, während die strukturelle Verteilung der ersten Periode negativ beeinflußt wird. Zusammenfassend läßt sich sagen, daß der Aggregate Investment Approach auf einen durchaus relevanten Sachverhalt, nämlich die zeitliche Entwicklung der Verteilungsniveaus, abstellt, strukturelle Überlegungen aber völlig vernachlässigt.

Es ist zu betonen, daß mit dem Finanzierungsinstrument der staatlichen Kreditaufnahme nicht a priori eine definitive Entscheidung über das zeitliche Auftreten vertellungsrelevanter Wirkungen verbunden ist, sondern dies von den speziellen Umstände abhängt. Hierzu kann auf die Ausführungen über die Investitionsmöglichkeiten verwiesen werden. Geht man hypothetisch vom klassischen Wirkungsmechanismus aus, so sind je nach Produktivität der Staats ausgaben[207] Wachstumseinbußen und damit verringerte Verteilungs potentiale in den Folgeperioden zu erwarten. Die strukturelle Verteilung der Ausgangs periode, die dann keine Änderungen erfährt, kann bei ceteris-paribus-Betrachtung in den Folgeperioden konstant bleiben, sofern die "verdrängten" Investitionen wieder auf die alte Quote zurückkehren[208], wobei diese Konstanz der Verteilungsstruktur dann mit einem geringeren absoluten Verteilungsniveau verbunden ist. Die Geldpolitik legt, so sie diese Wirkungsmechanismen tatsächlich steuern bzw. erzwingen kann, den Zeitpunkt der Belastung und die Träger dieser Belastung fest. Bei konstant bleibender Verwendungsstruktur tragen alle entsprechend der strukturellen Verteilung an diesen Wachstums verlusten. Treten diese Effekte dagegen in der Phase der Schuldaufnahme ein, gehen sie verteilungsmäßig ausschließlich zu Lasten der Arbeitnehmer.

Auch wenn man die praktische Bedeutung dieser Überlegungen als nicht sonderlich groß einschätzen sollte, kommt in der Verbindung von Niveau- und Strukturbetrachtung das generelle verteilungspolitische Problem zum Ausdruck, inwieweit Wachstumspolitik die beste Verteilungspolitik sei. 


\section{C) MIKROÖKONOMISCHE ANSÄTZE}

1. Ric ardo

a) Äquivalenz theorem

Schon an anderer Stelle wurde auf die Altehrwürdigkeit der Diskussion um die alternativen Finanzierungsmaßnahmen Steuer und Anleihe verwiesen. Einer der am längsten zurückliegenden und dabei immer noch diskutierten Beiträge stellt Ricardos Äquivalenztheorem dar, das das zeitliche Auftreten von Belastungseffekten für das Individuum bei (quantitativ sich entsprechender) Steuer - und Anleihefinanzierung staatlicher Ausgaben miteinander vergleicht. Dabei kommt Ricardo zum Ergebnis, daß ein Individuum diesen beiden Alternativen indifferent gegenüberstehen müsse[209]. Für das einzelne Individuum stellt sich die Entscheidungssituation derart, daß es zwischen einer einmaligen Steuerzahlung oder aber (bei Kreditfinanzierung der Staatsausgaben) der für die Verzinsung der staatlichen Anleihe notwendig werdenden zukünftigen Steuerzahlungen wählen kann. Die Ricardianischen Annahmen unterstellen eine klassische Modeltwelt vollkommener Information und entsprechend vollkommener Kapitalmärkte[210]. Die erste Annahme ermöglicht die notwendigen Informationen für das einzelne Wirtschaftssubjekt, die zweite die beliebige Transformation von Zahlungsströmen im Zeitablauf. Führt die Steuerfinanzierung $z u$ einer unausweichlichen Belastung in der ersten Periode, wird im zweiten Fall diese Belastung entsprechend der Zinsverpflichtungen über die Zeit verteilt. Beide formal sich unterscheidenden Alternativen sind aber nach Ricardo ökonomisch äquivalent. Diskontiert das Individuum nämlich seine zukünftigen Zinsbelastungen $a b$, so muß dies unter den Annahmen des Modells einer einmaligen Steuerzahlung äquivalent sein. Umgekehrt bestünde im Falle der Steuerfinanzierung die Möglichkeit, durch eine individuelle Kreditaufnahme diese Belastung entsprechend der Zinsverpflichtung zu verteilen. Voraussetzung dafür ist allerdings ein vollkommener Kapitalmarkt, der es allen Individuen ermöglicht, diese Transaktionen vorzunehmen, indem eine völlige Mobilität längs der Zeitachse gewährleistet wird. Um diese Problemstellung von Fragen der intergenerativen Verteilung freizuhalten, sei eine konstante, ewig lebende Bevölkerung angenommen, für die zudem identische Zeitpräferenzen unterstellt werden.

b) Gründe für differentielle Effekte

$b_{1}$ ) Mangelnde Information und Illusion

Insbesondere Gandenberger hat die Diskussion dieses Äquivalenztheorems wiederaufgenommen und die Argumente pro und contra systematisiert[211]. Er untersucht dabei in streng klassischem Rahmen (er selbst spricht von neoklassischem) die Gründe, weswegen sich unterschiedliche Effekte bei den 
beiden ökonomisch eigentlich äquivalenten Alternativen Steuer und Anleihe ergeben, wobei er zwei grundlegende Ursachenkomplexe behandelt. Der erste sind die Informations - und Illusionsprobleme. Die fehlende Information kommt im Problem der exakten Be- und Zurechnung der notwendig werdenden Zinssteuern zum Ausdruck.

Das Illusionsargument geht darüber hinaus, da auch bei gegebener Information aufgrund unterschiedlicher Zeithorizonte ein individuell andersartiges Verhalten zu beobachten ist. Wohl für beide Fälle läßt sich die Beurteilung der Staatsverschuldung durch Ricardo selbst anführen, "It is a system which tends to make us less thrifty, to blind us to our real situation"[212]. Liegt bei fehlender Information ein Verhalten des Individuums vor, wie wenn sich der Staat nicht verschuldet hätte (und damit keine Steuerverpflichtung entstanden wäre), da sich keine Änderung im individuellen Datenkranz ergeben hat, so ist das Illusionsargument anders zu erklären. Hier trifft das Individuum seine Verwendungsentscheidungen insoweit unter falschen Annahmen, als es die auf sich zukommenden Verpflichtungen ignoriert bzw. falsch einschätzt und nicht abdiskontiert. In den Auswirkungen auf das mikroökonomische Verhalten sind dabei keine Unterschiede zu sehen, in beiden Fällen fühlt sich das Individuum bei staatlicher Verschuldung nicht belastet.

Im Zuge der Illusionsdiskussion ist auf Puviani zu verweisen, der inhaltlich weitgehend mit Ricardo übereinstimmt, aber zusätzliche Erklärungshypothesen für das Auftreten von Illusionen anbietet. So erzeugt das Halten von Vermögensbesitz im Falle einer Staatsverschuldung ein subjektives Gefühl des größeren Reichtums, das dazu führt, daß das Individuum trotz Abdiskontierung der zukünftigen Zinsverpflichtungen der Anleihefinanzierung gegenüber der Steuerfinanzierung den Vorrang gibt, da es im ersten Fall den Vermögensbestand erhalten kann[213]. Allerdings treffen diese Aussagen nur unter gelockerten Annahmen $\mathrm{zu}$, so kann die Prämisse des vollkommenen Kapitalmarkts nicht aufrechterhalten werden, da andernfalls alle Individuen durch zusätzliche Verschuldung dieser "Vermögensillusion" nachgehen könnten. Gandenberger klassifiziert den Beitrag Puvianis, indem er nicht mehr von einem einfachen illusionistischen Verhalten spricht: "Man wird die von Puviani geschilderte Präferenz der Vermögenskontrolle vielmehr einem Rationalverhalten bei Unsicherheit und bei marginalen Zeitpräferenzraten, die vom Kapitalmarktzins abweichen, zuschreiben müssen"'[214].

Die exakte Trennung der Illusions - und Informationsargumente, die gleichgerichtete Auswirkungen auf das Konsumverhalten mit sich bringen, fällt schwer. Letztendlich reduziert sich aber auch das Phänomen der Illusion auf einen Informationsmangel, da bei entsprechender "Aufklärung" - nämlich bezüglich des Verhaltens (nicht der objektiven Tatbestände) - eine entsprechende Verhaltensänderung erreicht werden müßte. In jedem Fall sind die oben angesprochenen differentiellen Verhaltenseffekte auf nicht hinreichende Information zurückzuführen. Da diese Illusionsproblematik innerhalb der individualistischen Ansätze eine nicht unerhebliche, die Verteilungsergebnisse determinierende Rolle spielt, wurde hier stärker darauf eingegangen, wobei die pragmatischen Probleme noch völlig unberührt blieben. 
Der zweite Begründungskomplex führt zu unmittelbar verteilungsrelevanten Zusammenhängen zurück. Es wird, wie schon oben bei Darstellung der Vermögensillusion Puvianis, davon ausgegangen, daß der Kapitalmarkt nicht mehr in dem Sinne vollkommen ist, daß für alle Wirtschaftssubjekte identische Zinssätze gelten[215], doch bleibt die Annahme vollkommener Information bestehen. Die weitergehende, verteilungsrelevante Annahme ist, daß die Individuen bzw. bestimmte Subgruppen unterschiedliche Zeitpräferenzen aufweisen. Dies hat zur Folge, daß im Rahmen des oben gebrauchten RicardoModells zur Abdiskontierung der zukünftigen Zinssteuerverpflichtungen unterschiedliche Diskontraten Verwendung finden, damit aber auch unterschiedliche Belastungen (in Barwerten ausgedrückt) zustande kommen. Je höher diese subjektive Zeitpräferenzrate über der Verzinsung der Staatsverschuldung liegt, um so größer wird der Vorteil des Betreffenden gegenüber einer Steuerfinanzierung sein. Verteilungspolitisch bedeutsam werden diese Zusammenhänge dadurch, daß man für die ärmeren Bevölkerungsschichten von einer höheren Zeitpräferenzrate als bei den einkommensstärkeren Schichten ausgehen kann[216]. Für diese Gruppe besteht damit bei einer Anleihefinanzierung (scheinbar) die Möglichkeit, durch von ihnen favorisierten Gegenwartskonsum ein insgesamt (intertemporal) gesehen höheres Nutzenniveau zu erreichen. Dabei ist ein Rückgriff auf Nutzenvorstellungen nicht vermeidbar, da eine subjektive Komponente durch die individuellen Diskontierungsraten ins Spiel kommt. Geht man analog zum kreislauftheoretischen Vorgehen zu einer Zwei-KlassenBetrachtung über, so könnte man sich mit der rein qualitativen Annahme behelfen, daß die Gruppe der geringer Verdienenden eine höhere Gegenwartspräferenz hat.

\section{Verteilungspolitische Beurteilung Ricardos}

Eine verteilungspolitische Schlußfolgerung[217] aus diesem Umstand wäre, daß ein möglichst großer Teil der Staatsausgaben über Anleihen finanziert werden sollte, da dadurch positive Verteilungseffekte erzielt werden, erreichen doch die Schichten mit niederen Einkommen aufgrund ihrer Präferenz für Gegenwartskonsum eine optimalere intertemporale Verteilung ihres verfügbaren Einkommens. Der erste gravierende Einwand ist das Problem der Rationalität solcher Gegenwartspräferenzen. Hier muß an die Argumentation in Verbindung mit der Illusionsproblematik erinnert werden.

Berücksichtigt man den Aspekt der endlichen Lebensdauer der Individuen, so kommt das Problem der Vererbung hinzu. Die hier vorgeschlagene Möglichkeit räumt dem Individuum nicht nur größere Spielräume ein, sondern gestattet zudem die Möglichkeit eines negativen Erbes[218]. Dieser Umstand ist gerade für die besitzlosen Gruppen relevant, denen eine neue Dimension eröffnet wird, während bei anderen Gruppen auch anderes Vermögen zu konsumtiven $Z$ wecken zur Verfügung stünde. Üblicherweise zielen verteilungspoli- 
tische Maßnahmen darauf ab, die gütermäßige Verteilung zugunsten bestimmter Gruppierungen zu beeinflussen, was bei einer Zeitpunktbetrachtung zu einer Belastung anderer Gruppen führt[219]. Im Gegensatz zu einer solch horizontal, d.h. interpersonell ausgerichteten Verteilungspolitik haben wir es bei Ricardo mit einer intrapersonellen und vertikal, d.h. intertemporal orientierten $\mathrm{zu}$ tun[220]. Zwar ist nicht bestreitbar, daß durch die staatliche Schuldaufnahme innerhalb der Prämissen dieses neoklassischen Modells die Bewegungsfrelheit der Individuen vergrößert wird, doch steht und fällt die Rationalität einer solchen individuell orlentierten Verteilungspolitik mit der der als Datum verwendeten subjektiven Zeitpräferenzraten. Wenn andererseits der Staat die Aufgabe zugesprochen bekommt, auch übergeordnete Zielsetzungen wie das Wohl der kommenden Generationen aufgrund der Kurzsichtigkeit des Einzelnen korrigierend zu berücksichtigen, so muten solche auf die Rationalität und entsprechende Informationen des einzelnen Individuums gründenden verteilungspolitischen Vorschläge recht irreal an[221]. Selbst wenn Vorbehalte gegenüber der Rationalität und Informationsausstattung individueller Zeitpräferenzen nicht angebracht wären, muß man doch zum Schluß kommen, daß es sich bei Bemühungen dieser Art um eine sehr konservative Verteilungspolitik handeln muß, da die interpersonelle Einkommensverteilung davon unberührt bleibt.

Mit diesen Feststellungen ist ein weiteres grundsätzliches Problem des Ricardo-Ansatzes, nämlich die Annahme der Gültigkeit der klassischen Schuldtheorie, noch nicht einmal angesprochen. Räumt man aber auch die Möglichkeit anderer makroökonomischer Wirkungsabläufe ein, die in Form inflationärer Impulse zu einer Belastung in der Gegenwart führen, so wird die Unhaltbarkeit obiger Betrachtungsweise deutlich. Dabei stellt gerade die Lautlosigkeit einer solchen inflationären Finanzierung größte Informationsprobleme, von einer Erweiterung der individuellen Freiheitsspielräume kann dann nicht mehr die Rede sein. Da aber in einem solchen Falle die von diesem Ansatz unterstellten Verteilungswirkungen gerade in ihr Gegenteil verkehrt werden, ist eine verteilungspolitische Anwendung dieser Gedankengänge auch nur bei der Möglichkeit einer inflationären Wirkung der staatlichen Schuldaufnahme, und diese kann nie ausgeschlossen werden, als höchst fragwürdig zu bezeichnen.

\section{Buchanan}

\section{a) Grundlegende Thesen}

Nach der dogmengeschichtlich begründeten Einleitung soll nun auf den bekanntesten und streitbarsten Vertreter dieser individualistischen Richtung eingegangen werden. In prononcierter Abwendung von der makroökonomischen $\mathrm{Be}-$ trachtungsweise stellt Buchanan in seinen zahlreichen Beiträgen[222], die sich zentral mit den intertemporalen Effekten der staatlichen Verschuldung befassen, explizit auf das Individuum ab. Den Vertretern der von ihm als Neue 
Orthodoxie gekennzeichneten Richtung hält er vor, daß mit diesen auf organizistischen Gesellschaftsvorstellungen basierenden Aussagen keine sinnvollen Antworten gegeben werden könnten[223]. Die starke Resonanz, die Buchanan mit der Veröffentlichung seiner "Public Principles of Public Debt" im Jahre 1958 fand, ist allein schon aus seiner programmatischen Absicht erklärbar, die herrschende Lehrmeinung zu erschüttern bzw. zu widerlegen, was ihm von seiten Tobins den Vorwurf der Bilderstürmerei einbrachte[224]. Das ergebnisorientierte Vorgehen Buchanans, der die Aussagen der Neuen Orthodoxie in drei Thesen zusammenfasst und dazu konträre Gegenthesen zu beweisen sucht, findet in der sich anschließenden Diskussion teilweise seine Entsprechung[225].

Im Mittelpunkt seiner Angriffe steht die von der Neuen Orthodoxie vertretene Ansicht, daß durch eine staatliche Kreditfinanzierung keine Lastverschiebung (im Gegensatz zur privaten Verschuldung) möglich sei, da der Ressourcenverbrauch in der Verschuldungsphase stattfinden müsse. Buchanan sieht dagegen die Analogie zur privaten Verschuldung gewahrt, da ein Ressourcentrans fer in den öffentlichen Sektor möglich sei, ohne daß dabei Zahlungen bzw. Nutzeneinbußen in dieser Periode erforderlich sind.

Vor der inhaltlichen Auseinandersetzung mit Buchanans Thesen seien seine grundlegenden Annahmen dargelegt. Buchanan unterstellt bei all seinen Deduktionen eine klassischen Mechanismen gehorchende Wirtschaft[226]. Konstitutiv für eine "real debt" ist dabei der gleichzeitige (freiwillige) Verzicht auf Güterverwendung durch den privaten Sektor[227]. Innerhalb dieses theoretischen Rahmens stellt Buchanan das Individuum in den Mittelpunkt, um dessen Belastungen durch den Ressourcentransfer in den öffentlichen Bereich im Zeitablauf zu verfolgen. Äußerer Ausdruck dieser Belastung sind dabei die Steuerzahlungen zur Finanzierung von Zinsen und Tilgungen, die mit einer Nutzeneinbuße verbunden sind, während sich in der Schuldaufnahmephase keine solche Einkommensbelastung feststellen läßt. Dieses Rekurrieren auf Nutzenvorstellungen ist das wohl bekannteste Charakteristikum für Buchanans Ansatz geworden. So klassifiziert Gandenberger in seinem Überblicksartikel diesen Ansatz mit Buchanans Einverständnis als Utility Approach[228]. Dennoch kommt auch bei Verwendung dieses Indikators dem verfügbaren Einkommen als Maßstabsgröße die entscheidende Bedeutung zu. Daß eine Reduktion der verfügbaren Einkommen mit einer Nutzeneinbuße verbunden ist, entspricht auch unserer Behandlung der Sekundärverteilung als verteilungspolitischem Indikator.

Aufgrund des ordnungspolitischen Standpunktes Buchanans ist der Hinweis wichtig, daß er keine Beziehung zwischen dem Zensiten und der zu finanzierenden Ausgabe herstellt. Buchanan geht also von einer Bruttobetrachtung aus. Dies ist mit den unterstellten Budgetentscheidungsstrukturen erklärbar, da Buchanan annimmt, daß das Individuum keine Einflußmöglichkeiten besitzt. Damit kann dem Vorwurf entgegengetreten werden, daß eine Steuerzahlung dann nicht mit einer Nutzeneinbuße verbunden sein müsse, wenn eine Identifizierung des Individuums mit den Staatsausgaben erfolgt[229]. Darüber hinaus geht Buchanan davon aus, daß die Steuerzahlungen infolge einer staatlichen Schuldaufnahme 
nicht antizipiert werden, er unterstellt bei den Individuen Schuldillusion, da diese an der Entscheidung über die Finanzierungsart nicht beteiligt sind[230].

\section{b) Kosten im Entscheidungsprozeß}

Konstitutiv für jede Auseinandersetzung mit Buchanan muß eine Klärung und Abgrenzung der Begriffe sein, mit denen er verteilungsrelevante Sachverhalte zu erfassen sucht. Damit sind die Begriffe der Last und der diversen Kosten bei diesem Autor angesprochen. Nach Buchanans eigener Aussage ist die Verwirrung im Hinblick auf den Kostenbegriff kaum einmal evidenter hervorgetreten als bei der Diskussion um die Inzidenz der Staatsverschuldung[231], wobei er einräumt, selbst dazu beigetragen zu haben[232]. Es soll hier nicht die historische Entwicklung seines Kostenbegriffs aufgerollt werden, dazu sei auf die Arbeit von Kiepe verwiesen[233], sondern dessen letzte Fassung im Sinne der von Buchanan in "Cost and Choice" bezogenen Position der subjektivistischen Wertlehre dargelegt werden. Buchanan wendet sich scharf gegen objektive Kosten im Sinne neoklassischen Modelldenkens, welches sich im starren Rahmen von ceteris-paribus-Bedingtheiten nicht in der Lage zeige, dem selbstgesteckten Ziel der erklärenden Aussage[234] gerecht $\mathrm{zu}$ werden. Unter Berufung auf Vertreter der österreichischen Schule richtet Buchanan sein Augenmerk auf die Entscheidungssituation des Individuums, in der die relevanten und nur von diesem Entscheidungsträger erlebbaren und nicht objektivierbaren Kosten anfallen[235]. Buchanan bezieht sich bei seinen Ausführungen immer auf diese subjektiven Kosten, die dem Nutzenkalkül der jeweils betroffenen Individuen entspringen.

Dabei gilt es im Verlauf eines Entscheidungsprozesses zwei Formen dieser subjektiven Kosten zu unterscheiden: Zum einen die entscheidungsbeeinflussenden Kosten (choice influencing costs), zum anderen die entscheidungsabhängi gen Kosten (choice influenced costs)[236]. Die unmittelbar bei der Entscheidung auftretenden Kosten sind dadurch gekennzeichnet, daß sie nur beim Entscheidungsträger (dies kann eine Einzelperson aber auch eine Gruppe sein) zum Zeitpunkt der definitiven Wahl anfallen. Diese Kosten sind aufgrund ihres Nutzencharakters nicht meßbar, so ist es einem Außenstehenden nicht einmal möglich, alle Faktoren, die in das Entscheidungskalkül eingehen, in Erfahrung zu bringen. Sie sind also in ihrer - für den Außenstehenden nicht beobachtbaren - Gesamtheit Ausdruck all der Momente, die der aktuellen Entscheidung Widerstände entgegensetzen. Dementsprechend ist der Charakter der choice influencing costs der einer ex ante Größe, d.h. das subjektive Nutzenkalkül umfaßt eine Vielzahl von Antizipationen.

Mit der Auswahl einer bestimmten Alternative, mit der ein Entscheidungsprozeß abgeschlossen wird, ist diese Kostenart nicht länger von Interesse. Ihr Einfluß erschöpft sich also in den unterschiedlichen Widerständen, die den einzelnen Wahlmöglichkeiten entgegengebracht werden. 
Im unmittelbaren Anschluß und in den weiteren Folgeperioden gilt es, den zweiten Kostentyp zu beachten, den der choice influenced costs. Neben dieser eindeutigen Trennung der beiden Kostenarten nach ihrem unterschiedlichen temporalen Auftreten muß man den unterschiedlichen Kreis der $\mathrm{Be}-$ troffenen beachten. War dies im ersten Falle immer der oder die Entscheidenden, so können die im Gefolge einer Entscheidung anfallenden Kosten von vornherein nicht auf einen bestimmten Personenkreis beschränkt werden. In den entscheidungsbeeinflußten Kosten kommen all die Faktoren zum Ausdruck, die beliebige Individuen infolge einer bestimmten Entscheidung in ihren Nutzenniveaus beeinflussen. Dabei können als Bemessungsgrundlage objektiv feststellbare Größen wie Zahlungsströme herangezogen werden.

c) Kosten der Staatsverschuldung

$\left.c_{1}\right)$ Entscheidungsbeeinflussende Kosten

Es sollen nun diese beiden für Buchanan mit jedem Entscheidungsprozeß untrennbar verbundenen Kostenarten auf ihre verteilungspolitische Aussagefähigkeit am Beispiel der Staatsverschuldung überprüft werden. Dazu gehen wir von der Entscheidungssituation aus, die sich bei dem Entschluß zu einer kreditfinanzierten Staatsausgabe stellt. In der Entscheidungsphase der Schuldaufnahme müssen die entscheidungsbeeinflussenden Kosten bei den Entscheidungsträgern festgestellt werden, die sich für diese Finanzierungsalternative entschließen. Bei der Ermittlung dieser Kosten müssen ihrem ex ante Charakter entsprechend Vorstellungen über die Auswirkungen der Entscheidung bestehen. Dies ergibt das qualitative Gerüst dieser Kostenart, die Bewertung dieser Kosten kann aber nur vom Entscheidungsträger vorgenommen werden. Naheliegenderweise wird die Bewertung dieser Informationen, d.h. ihre Transformation in Nutzengrößen, mit der Zusammensetzung der Gruppe der Entscheidungsträger variieren. Diese Bewertungsproblematik spielt bei unserer Fragestellung aber keine Rolle, da wir von einer gegebenen Ausgaben- und Finanzierungsentscheidung ausgehen.

Durch das Aufzeigen der entscheidungsbeeinflußten Kosten unter Betonung des Verteilungsaspektes kann aber das Informationsniveau der Entscheidungsträger, d.h. der Regierung und des Parlaments, verbessert werden. Entsprechend interessieren hier nur noch die choice influenced costs, stellen diese doch auch einen Teil der insgesamt komplexen Entscheidungshemmnisse dar.

\section{$c_{2}$ ) Entscheidungsbeeinflußte Kosten}

Die durch die Entscheidung verursachten Kosten sind im Falle der staatlichen Verschuldung nach Buchanan erst in den Folgeperioden in Gestalt der Zinsund Tilgungsverpflichtungen zu erwarten, wobei diese Kosten bei einem völlig anderen Personenkreis als dem der Entscheidungsträger anfallen können. Zwar 
ist auch hier die endguiltige Transformation in Nutzengrößen nur den betroffenen Individuen möglich, aber die monetäre Steuerbelastung kann als Hilfsgröße zu ihrer Erfassung herangezogen werden[237]. Hierbei gilt es, zwei Aspekten besondere Beachtung zu schenken: Zum einen sieht Buchanan keinerlei verteilungsrelevante Wirkungen in der Schuldaufnahmephase bzw. verweist sie in den vernachlässigbaren Bereich der Sekundäreffekte[238], zum anderen betrachtet er nur die Steuerzahlungen als ursächlich für die Verteilungswirkungen, wohingegen die Zinszahlungen als Folge der Vermögensbildung anzusehen sind. Zwischen diesen beiden Sachverhalten darf keine Kausalität hergestellt werden, da der Entscheidungsprozeß bezüglich der Finanzierungsform von Staatsausgaben völlig getrennt vom individuellen Entscheidungsprozeß bezüglich Höhe und Ort der Vermögensbildung verläuft[239][240]. Die verteilungs politisch relevante Größe sind also allein diese Folgekosten, wobei die strenge Trennung der Steueraufbringung von den Zinszahlungen hervorzuheben ist[241]. Durch diese scharfe Trennung des Problems der individuellen Vermögensanlage einerseits von der Entscheidung zur Finanzierung mittels staatlicher Anleihen andererseits kommt Buchanan zum Ergebnis, daß in der Phase der Schuldaufnahme keine Belastung erfolge. Das Individuum kennt also keine Abdiskontierung zuküntiger Belastung wie bei Ricardo, Buchanan geht vom $\mathrm{Zu}-$ stand der Schuldillusion als Normalfall aus.

Unabhängig vom unterstellten klassischen Wirkungsmechanismus muß aber auch bei Buchanan ein anderes makroökonomisches Aggregat durch die staatliche Kreditnahme zurückgedrängt werden. Da es sich dabei aufgrund der speziellen Annahmen Buchanans immer um die privaten Investitionen handelt, wurde in der Literatur der verdrängte private Kreditnehmer als der eigentliche Träger der Belastung angesprochen[242]. Nach Buchanan handelt es sich dabei um strukturelle, über das anonyme Marktgeschehen ablaufende Effekte, die sich gegenseitig kompensieren können[243]. Indem aber solche Vorgänge als "secondary repercussions" abgetan werden, da sie über das anonyme Marktgeschehen ablaufen, müssen gegenüber der verteilungspolitischen Aussagefähigkeit dieser Überlegungen doch erhebliche Zweifel angemeldet werden[244].

\section{d) Verteilungspolitische Beurteilung.}

Es ist nochmals zu betonen, daß dieses Ergebnis einer möglichen Lastverschiebung durch den unterstellten Wirkungsablauf determiniert ist[245], wonach im Umfange der staatlichen Kreditaufnahme freiwilliger Nachfrageverzicht vom privaten Sektor geübt wird. Buchanan behandelt in seinen "Public Principles of Public Debt" ausführlich auch den Fall, daß inflationäre Effekte als Folge staatlicher Verschuldung auftreten. Dann konstatiert auch er eine in der Gegenwart liegende Belastung, deren strukturelle Verteilungseffekte er mit denen einer indirekten Steuer auf die Kassenhaltung vergleicht[246]. 
So sind bei Buchanan die unterstellten Wirkungsabläufe der entscheidende Faktor für die Verteilungsergebnisse, nicht aber eine besondere Definition der Belastung. Wenn etwa Wittmann behauptet: "Mit seinem psychologisch orientierten Begriff der Lastverschiebung steht Buchanan in diametralem Gegensatz zur realen (güterwirtschaftlichen) Argumentation der Klassike r"[247], so wird hier fälschlicherweise der Eindruck erweckt, daß die Ergebnisse die Folge einer bestimmten Begriffsfassung sein könnten[248]. Daß Buchanan auf subjektive Größen abstellt, bringt zumindest für die verteilungspolitische Beurteilung seiner Ergebnisse keine besonderen Schwierigkeiten mit sich. Die Betonung der Freiwilligkeit im Falle der Anleihen im Gegensatz zum Zwangscharakter der Steuererhebung übersieht zumeist, daß die Begründung für diese Unterscheidung darin zu sehen ist, daß die Anlage in staatlichen Papieren als Teil der allgemeinen Entscheidung zur Vermögensbildung gesehen werden muß, die mit dem Ressourcenübergang in den staatlichen Bereich keinen Sachzusammenhang aufweist, die gütermäßig notwendigen Verdrängungseffekte sich aber über das anonyme Marktgeschehen mit seinen Preisregulatoren einspielen. Man wird den Intentionen Buchanans nicht gerecht, wenn man seine Betrachtungsweise wie in den oben genannten Fällen dadurch charakterisiert, daß er sich um die Erfassung psychologischer Phänomene bemühe. Das Abstellen auf Nutzengrößen ist durch den methodologischen Standpunkt Buchanans erklärbar.

Von seinem Untersuchungsziel her stellt auch Buchanan auf Verteilungsprobleme ab[249]. Das von ihm zugrundegelegte Modell hat aber zur Folge, daß in der Phase der Schuldaufnahme keine Effekte zu verzeichnen sind. In kreislauftheoretischer Sicht ist eine solche Vernachlässigung der Verteilungseffekte in der Phase der Schuldaufnahme zu rechtfertigen, da Buchanan klassische Wirkungsmechanismen als gegeben annimmt. Eine verteilungstheoretisch fundierte Untersuchung müßte aber auf die Zinssatzsteigerungen und die daraus resultierenden Verteilungseffekte eingehen[250]. Der Verweis auf das Marktgeschehen, in dessen Anonymität sich Verlust- und Gewinnpositionen ausgleichen werden, kann für eine verteilungspolitische Aussage nicht genügen.

Die Kostenkonzeption Buchanans vermag keinen entscheidenden verteilungspolitischen Beitrag zu leisten. Letztendlich stellen die choice influenced costs keine neue Kategorie dar, müssen doch auch sie auf die Veränderungen der Sekundärverteilung zurückgreifen. Die choice influencing costs sind für unsere verteilungspolitische Fragestellung nicht unmittelbar von Interesse, doch führt diese Trennung der Entscheidungsprozesse zu einem methodisch klaren Erkennen der eigentlich relevanten Faktoren. Ein wesentlicher Beitrag Buchanans ist also in der korrekten Behandlung der Zinszahlungen einerseits und der Steuern andererseits zu sehen, indem er die Pseudo-Kausalität des Transferansatzes aufgrund seiner entscheidungstheoretisch fundierten Überlegungen zurückweist.

Das Aufzeigen der Steuerbelastung geschieht zwar in intertemporaler Sicht in zutreffender Weise, bleibt aber bei einer formalen Inzidenzbetrachtung stehen. Daß die Steuererhebung zur Finanzierung staatlicher Zinszahlungen nicht un- 
bedingt zu einer effektiven Belastung führen muß, wurde oben aufgezeigt, da die Verwendungsart dieser Steuern für die effektive Inzidenz von Bedeutung ist. Auch für den klassischen Ansatz hätte man einige Überlegungen bezüglich der effektiven Inzidenzbelastung erwarten können. So ist die Frage zu stellen, ob die gesamte Diskussion durch die andersartige Terminologie nicht nur im rein Formalen verblieb und unübersichtlicher wurde. Buchanans Überlegungen stellen also eine vom Ansatz her durchaus sinnvolle Betrachtungsweise dar, die bezüglich der materiellen Ergebnisse allerdings etlicher Ergänzungen bedürfte. Hier ist zum einen an die Vernachlässigung der Effekte in der Schuldaufnahmephase, zum anderen an die rein formale Inzidenzbetrachtung der Zinssteuern zu denken. Darüber hinaus bleiben die unterstellten Wirkungsmechanismen einseitig, die von Buchanan als Normalfall unterstellte "real debt" hat im gesamten Spektrum staatlicher Verschuldungsmöglichkeiten eher Ausnahmecharakter.

3. Ansätze zur Erklärung intergenerativer Verteilungseffekte

\section{a) Bowen-Davis-Kopf}

Während Buchanan bei seinen Überlegungen lediglich den Umstand der intertemporalen Verschiebbarkeit der Belastung durch staatliche Ressourcenbeanspruchung herausarbeitet, im eigentlichen Wortsinne aber keine intergenerativen Effekte aufzeigt[251], bemühen sich die Autoren Bowen, Davis und Kopf[252] innerhalb des auch von Buchanan verwendeten analytischen Rahmens explizit um eine auf die einzelne Generation abstellende Betrachtung. Ihr erklärtes Untersuchungsziel ist der Nachweis, daß eine intergenerative Lastverschiebung auch dann möglich ist, wenn die erste Generation in der Phase der Schuldaufnahme die staatlichen Papiere aus zusätzlicher Ersparnisbildung erwirbt. Die Betonung der Möglichkeit einer solchen Verschiebung ist von Bedeutung, da die Autoren damit einige restriktive Annahmen begründen. War bei Buchanan die Belastung durch eine Reduzierung des verfügbaren Einkommens einer Periode charakterisiert, so stellen Bowen/Davis/Kopf auf die Beeinträchtigung des Lebenseinkommens ab. Dies ist die unmittelbare Konsequenz der intergenerativen Betrachtung, die das Setzen einer zeitlichen Zäsur in Gestalt der Lebensdauer einer Generation erforderlich macht. Eine intergenerative Verschiebung der Belastung ist nach Bowen/Davis/Kopf dann erreicht, wenn das Lebenseinkommen einer Generation von der staatlichen Schuldaufnahme unberührt bleibt.

Die Beweisführung der Verschiebbarkeit basiert darauf, daß eine Generation[253] zu Ende ihrer Lebenszeit die staatlichen Papiere an die nachfolgende Generation veräußern und damit ihren Lebenszeitkonsum aufrechterhalten kann. Die Entschädigung für den zeitlichen Konsumaufschub wird mit den staatlichen Zinszahlungen als̀ abgegolten angesehen. Allerdings partizipiert jede Generation in Form der anteiligen Zinsverpflichtungen an der "Last"; in dieser Hin- 
sicht besteht kein Unterschied zur Analyse Buchanans[254]. Ein solcher kommt erst durch die Einführung sich überlappender Generationen zustande. Daneben muß bei der älteren Generation der Wunsch bestehen, kein Erbe $\mathrm{zu}$ hinterlassen[255].

So geht der Ansatz von Bowen-Davis-Kopf nicht wesentlich über die formalinzidenztheoretische Analyse Buchanans hinaus, wenn sie auch auf den Unterschied zwischen einer nur intertemporalen und einer intergenerativen $\mathrm{Be}-$ trachtung aufmerksam gemacht haben. Die Verschiebungsmöglichkeit der Last einer Staatsverschuldung beinhaltet prinzipiell nichts anderes als die schon von Ricardo aufgezeigte zusätzliche Wahlmöglichkeit eines negativen Erbes.

\section{b) Head}

Der differenzierteste mikroökonomische Ansatz zur Erfassung intergenerativer Effekte der Staatsverschuldung findet sich bei Head[256]. Ausgangspunkt seiner Analyse ist einerseits der grundlegende Ansatz Ricardos, andererseits die Kritik gegenüber den Ausführungen Buchanans, daß dieser lediglich eine Untersuchung der formalen Inzidenz durchgeführt habe. Die Überlegungen Heads sind für unsere Problemstellung von besonderem Interesse, da er sich explizit auf die Inzidenz-Terminologie bezieht, um so zu einer besseren Vergleichbarkeit der Effekte beizutragen.

Ein entscheidender Unterschied zu den Annahmen bei (und gleichzeitiger Vorwurf gegenuiber) Buchanan ist die je nach Informationsstand mehr oder weniger vollkommene Antizipation und Diskontierung zuküftiger Zinssteuerbelastungen mit entsprechenden Reaktionen der individuellen Verwendungsentscheidungen. Der Schritt von der rein formalen zur Bestimmung der effektiven Inzidenz wird nach Head dadurch vollzogen, daß die Verhaltensreaktionen der Individuen in der Phase der Schuldaufnahme berücksichtigt werden. In Weiterführung von Ricardo unterscheidet Head nicht nur zwischen Spar- und Konsumneigung, sondern spaltet diese Verhaltensannahmen noch weiter auf[257]. Als maßgebliche Faktoren zur Bestimmung der effektiven Inzidenz rücken damit die Spezifikation dieser Verhaltensfunktionen sowie die Informationsniveaus der Wirtschaftssubjekte in den Vordergrund. Im folgenden sollen lediglich die grundlegenden Kausalitäten anhand der von Head zugrundegelegten Verhaltens annahmen dargestellt werden[258], um so die Grundlage für eine Beurteilung der verteilungspolitischen Aussage der verschiedenen mikroökonomischen Ansätze zu schaffen. Head geht von vier verschiedenen Einkommensverwendungsmöglichkeiten der Wirtschaftssubjekte aus. Neben einer Verwendung für gegenwärtigen und zukünftigen Konsum differenziert er auch die Ersparnisbildung nach einem Motiv der reinen Akkumulation sowie dem der Vererbung. Die letztgenannte Verwendungsart ("bequests") stellt das Bindeglied zwischen den Generationen dar, da jede auf die zukünftige Generation entfallende Verpflichtung (in unserem Falle die Zinssteuerzahlungen) diese Größe mindert[259]. 
Antizipieren die Individuen zum Zeitpunkt der Schuldaufnahme (in mehr oder weniger vollständigem Umfange) die auf sie und ihre Erben zukommenden Belastungen in Form der Steuerzahlungen, so werden sie zu diesem Zeitpunkt mit entsprechenden Änderungen aller Verwendungsentscheidungen reagieren. Geht man von vollkommener Information aus, hängt die Einschränkung der jeweiligen Verwendungsart vom Verhältnis der Grenznutzen der einzelnen Verwendungsmöglichkeiten untereinander ab. Um konkrete Aussagen treffen zu können, müßten die entsprechenden Nutzenfunktionen der Individuen bekannt sein.

Der Unterschied zu Ricardos Analyse, der das Problem der endlichen Lebensdauer ausklammert, besteht darin, daß der Einkommensart "Vererbung" andere Gewichte beigemessen werden können als einer Ersparnis für persönliche spätere Verwendung. So betont auch Head, daß die angenommene Abdiskontierung der auf die Erben entfallenden Verpflichtungen nicht der in der Literatur häufig gebrauchten Annahme der "linear extensions of themselves" entspreche, da a priori keine Aussage darüber getroffen werde, in welcher Weise die Verwendungsarten gewichtet werden[260]. Mit dem Einbeziehen der Reaktionen der Individuen zum Zeitpunkt der Schuldaufnahme ist nach Head der Schritt von der formalen zur effektiven Inzidenz vollzogen. Durch die Beruicksichtigung einer Vielzahl von Verhaltensweisen sind die Ergebnisse nicht mehr eindeutig bestimmt, weswegen Head auch eine Reihe möglicher intergenerativer Effekte diskutiert. Spielt die Verwendungsart "Vererbung" beim Einzelnen keine Rolle, ist auch bei Head die Möglichkeit eines negativen Erbes im Falle einer Staatsverschuldung gegeben. Kommt den Erben in der individuellen Nutzenfunktion eine gleichwertige Bedeutung wie der lebenden Generation $\mathrm{zu}$, so entspricht die intertemporale Beurteilung der des Äquivalenztheorems. Bei extrem altruistischen Verhaltensannahmen besteht die Möglichkeit, die kommende Generation durch Erhöhung der Erbmasse von den Zinssteuerverpflichtungen zu befreien[261]. Dieser Fall stellt den Gegenpol des negativen Erbes dar, auch er verdeutlicht die zusätzlichen Wahlmöglichkeiten im Falle der Staatsverschuldung. Der Umfang der Belastung künftiger Generationen hängt zum einen vom Grad des Altruismus der unterstellten Nutzenfunktionen und zum anderen vom hier nicht weiter diskutierten Informationsstand der Individuen ab[262].

So zeigt Head auf, daß es zwar eine ganze Reihe von denkmöglichen intertemporalen und intergenerativen Effekten gibt, aber es scheint fraglich, ob eine dermaßen weitgehende Differenzierung der Verhaltensweisen noch plausibel ist. Diese Bedenken werden noch vergrößert, wenn man auf die damit verbundenen Informationsbedürfnisse verweist, die bei diesem Modellansatz verstärkt ins Gewicht fallen. Die Differenzierung nach unterschiedlichen Verwendungsmöglichkeiten für das private Einkommen mag in individueller Sicht eine wesentliche Rolle spielen, doch ist damit über die effektive Inzidenz der die Belastung verursachenden staatlichen Einnahmeinstrumente noch keine endgültige Aussage getroffen. Durch Berücksichtigung zusätzlicher privater Verhaltensreaktionen wird die effektive Inzidenzanalyse eher noch erschwert. Auch Head bleibt bei einer - allerdings komplizierteren - Form der formalen Inzidenz 
stehen. Auf Aussagen, die lediglich individuelle Möglichkeiten aufzeigen, lassen sich keine verteilungspolitischen Schlußfolgerungen aufbauen.

\section{D) SCHLUSSFOLGERUNGEN}

1. Aussagefähigkeit der mikroökonomischen Ansätze

Alle mikroökonomischen Ansätze zeigen eine recht enge Verwandtschaft mit dem Ausgangsmodell Ricardos; wesentlich darüber hinausgehende Ergebnisse werden nicht erbracht. Dabei ist zu berücksichtigen, daß mit jeder zusätzlichen Verhaltensannahme das ohnehin schon gravierende Informationsproblem an Bedeutung gewinnt. Unter diesem Aspekt erscheint der relativ einfache Ansatz Buchanans, der von einer völligen Schuldillusion als Normalfall ausgeht, durchaus von pragmatischem Interesse zu sein. Da der Vorwurf der nur formalen Inzidenzbetrachtung auf alle mikroökonomischen Ansätze zutrifft[263], liefert er mit seinem rein intertemporal ausgerichteten Modell den iberschaubarsten Beitrag.

Die Beschränkung auf das Aufzeigen rein intertemporaler Effekte scheint deshalb nicht einengend, weil das Abstellen auf intergenerative Wirkungen zu keinen operationalen Aussagen führt. Es ist eine nahezu beliebige Vielfalt an Effekten konstruierbar, wenn man das Phänomen der overlapping generation berücksichtigt, da jede Interaktion zwischen unterschiedlichen Generationen dann auch intergenerative Effekte mit sich bringt. Diese Effekte spielen sich aber iber das anonyme Marktgeschehen ab und resultieren aus individuellen Verhaltensentscheidungen. Damit wird das Bemühen problematisch, auf gesamtwirtschaftlicher Ebene intergenerative Effekte einer Staatsverschuldung aufzuzeigen. Es besteht nur die Möglichkeit, die Existenz solcher Effekte einzuräumen - eine verteilungspolitisch relevante Erfassung ist aber nicht gegeben.

Beispiel dafür ist die Untersuchung von Bowen-Davis-Kopf, deren Untersuchungsziel der Nachweis ist, daß eine Belastung künftiger Generationen möglich sei. Dieser Möglichkeitsnachweis gelingt den Autoren auch, doch lassen sich darauf keine allgemeingültigen Aussagen über die Entwicklung der intergenerativen Effekte gründen[264]. Allen mikroökonomischen Ansätzen ist der Rahmen der klassischen Schuldtheorie gemeinsam, womit in kreislauftheoretischer Sicht die Vernachlässigung der Verteilungseffekte in der Schuldaufnahmephase zu rechtfertigen wäre. Die Behandlung der dann zentralen Verzinsungsperiode geht aber bei keinem der Autoren über eine reine Formalinzidenzbetrachtung hinaus. Alle diese Ansätze stellen letztlich auf das Lebenseinkommen bzw. die Einkommensströme bestimmter Zeiträume ab, wobei aber iber die Entwicklung der gesamtwirtschaftlichen Verteilungsniveaus keine Aussage getroffen wird. Es ist deshalb naheliegend, die Ergebnisse der mikroökonomischen Ansätze mit den Überlegungen des makroökonomischen Wachstumsansatzes $\mathrm{zu}$ verbinden. Bei diesem Integrationsversuch werden wir 
uns auf den Ansatz Buchanans beziehen, da von ihm dazu eine Stellungnahme vorliegt, zudem sein Ansatz den Vorteil der Überschaubarkeit bietet.

2. Integrierende Betrachtung des mikroökonomischen und makroökonomischen Ansatzes

Folgt man dem mikroökonomischen Ansatz Buchanans einerseits, fügt aber die Überlegungen des Aggregate Investment Approach andererseits hinzu[265], so sieht man, daß bei Buchanan lediglich Fragen der Sekundärverteilung, bei der makroökonomischen Sicht aber ausschließlich die Entwicklung der Verteilungsniveaus angesprochen werden. Hier ist wiederum der Vorwurf der nur formalen Inzidenzsicht bei Buchanan zu erheben, der ausschließlich die Steuerzahlungen als einen das verfügbare Einkommen mindernden Faktor im Auge hat.

Koppelt man die beiden Aspekte, so können sich "Doppelbelastungen" ergeben, da einerseits das Verteilungspotential und andererseits die Verteilungsstruktur Veränderung erfährt. Es wäre aber auch denkbar, daß die zusätzlichen Steuern aus den über die Produktivitätseffekte der verdrängten privaten Investoren hinausgehenden Wachstumsimpulsen der staatlichen Investitionen "bezahlt" werden könnten[266]. Rückt Buchanan diese Wachstumsüberlegungen in seinen früheren Veröffentlichungen in den Bereich der Sekundäreffekte[267], so bezieht er in "Cost and Choice" zu den Vorwürfen der Vertreter des Aggregate Investment Approach Stellung[268].

Buchanan weist jede Verbindung zwischen der Entscheidung zur staatlichen Schuldaufnahme und den Überlegungen bezüglich der Höhe des zukünftigen Kapitalstocks und damit der Verteilungsniveaus zurück. Er räumt allerdings ein, daß dieser Zusammenhang für den Anleihezeichner von Bedeutung sein könne. Dieser habe zu entscheiden, ob er den Konsum oder die Investitionen zurückdränge, wobei im zweiten Falle durchaus eine Belastung der zukünftigen Erben zu erwarten sei[269]. Damit erkennt Buchanan zumindest auf mikroökonomischer Ebene die Zusammenhänge, auf die die Vertreter des Wachstumsansatzes für die Gesamtwirtschaft abstellen.

Diese Vertauschung der Entscheidungskriterien muß überraschen, denn für das Individuum steht zunächst allein die Vermögensbildung an sich im Vordergrund, die von den individuellen Präferenzen bestimmt wird. Die gesamtwirtschaftlichen Effekte aber soll das Individuum bei seinen Entscheidungen berücksichtigen[270], wohingegen der Staat als Anleihegeber diese wachstumspolitischen Effekte unberücksichtigt lassen und dafür auf die Entscheidungen der einzelnen Individuen vertrauen soll. Es kommt hinzu, daß die möglichen Wachstumsreduzierungen kaum in erkennbarer Form auf das einzelne Wirtschaftssubjekt durchschlagen, sondern die Gesamtheit der Wirtschaftssubjekte betreffen. 
Es ist hier die Frage aufzuwerfen, in welchem Rahmen diesen Überlegungen überhaupt Plausibilität zukommen kann, da selbst im neoklassischen Modelldenken zwar das Sparen die Investitionen determiniert bzw. begrenzt, Sparund Investitionsvorgang aber realiter auseinanderfallen. Man müßte also eine Konstellation unterstellen, in der der Investor (und nur dieser) gleichzeitig Konsumverzicht übt und diese Sparbeträge dem Staat zur Verfügung stellen kann. Sobald aber diese Vorstellung einer realwirtschaftlichen Identität aufgegeben wird, kann eine solche Argumentation wirtschaftspolitisch keine Relevanz mehr beanspruchen.

Dafür bietet sich an, diese Überlegungen in die choice influencing costs mit einzubeziehen, da durch die staatliche Haushaltsentscheidung diese Effekte verursacht werden[271].

\section{Beurteilung in kreislauftheoretischer Sicht}

Die Vertreter des Aggregate Investment Approach sind in ihrem Ansatz insofern den kreislauftheoretischen Überlegungen verwandt, als beide Betrachtungsweisen auf Veränderungen der Verwendungsstruktur in Folge zusätzlicher staatlicher Ansprüche abstellen. Wie die Kreislauftheorie der Verteilung aus Verschiebungen der Anteile von Konsum - und Investitionsgütern am Sozialprodukt auf interpersonelle Verteilungseffekte der Staatsverschuldung schließt, so werden bei Modigliani und anderen daraus Schlußfolgerungen bezüglich der intertemporalen Entwicklung der Verteilungsniveaus abgeleitet.

Oben wurde schon darauf verwiesen, daß bei Vertretern des Wachstumsansatzes häufig der klassische Wirkungsmechanismus stillschweigend unterstellt wird, was eine zwangsläufige Reduktion der Investition und damit eine relative Wachstumsminderung mit sich bringt. Wir können an dieser Stelle aber auf die Ausführungen zur interpersonellen Verteilung zurückgreifen, wo aufgezeigt wurde, daß ein solches Crowding-out keineswegs die Folge einer staatlichen Schuldaufnahme sein muß, ja nicht einmal als Normalfall zu erwarten ist. Demzufolge ist also auch eine Lastverschiebung nicht als Normalfall anzusehen. Davon unabhängig stellt aber das methodische Vorgehen dieses makroökonomischen Ansatzes insofern eine sinnvolle Ergänzung dar, als die rein strukturorientierte Verteilungssicht eine Relativierung erfährt.

Beurteilt man die mikroökonomischen Ansätze unter kreislauftheoretischen Gesichtspunkten, so ist diesen der Vorwurf nicht zu ersparen, daß die eigentlich relevanten Verteilungseffekte der Schuldaufnahmephase nicht erfaßt werden. Darüber hinaus konnte aus kreislauftheoretischer Sicht gezeigt werden, daß der effektiven Inzidenz der Zinssteuern keine wesentliche verteilungspolitische Relevanz zukommt. Oben wurden schon der Aussagekraft dieser Ansätze gegenuber erhebliche Zweifel angemeldet, da sie uiber das Beschreiben individueller Möglichkeiten nicht hinausgelangen, damit aber verteilungspolitische Fragestellungen nicht zulänglich beantworten können. 
Das Auseinanderfallen von formaler und effektiver Inzidenz ist lediglich bei Bowen-Davis-Kopf nicht zu beobachten, da diese Autoren - aus allerdings ganz anderen Motiven - von der Annahme ausgehen, daß im Umfang der staatlichen Kreditnahme zusätzliche Ersparnis gebildet wird. Dadurch werden sowohl die relativen Auswirkungen auf das Verteilungspotential als auch die Beeinflussung der personellen Einkommensverteilung durch inflatorische Impulse vermieden[272]. So sind die innerhalb dieses Ansatzes für die Individuen aufgezeigten Möglichkeiten nicht nur formaler Natur, sondern durchaus im Sinne der effektiven Inzidenz möglich. In individueller Sicht gilt natürlich immer, daß ein Vermögenswert zu erhöhtem Konsum verwendet werden kann, für das Individuum also eine Lastverschiebung möglich ist [273].

Will man die effektive Inzidenz einer staatlichen Schuldaufnahme im Zeitablauf erfassen, so müssen einerseits die Auswirkungen auf die Verteilungssituation der ersten Periode, darüber hinaus aber auch die Entwicklung dieser Primärverteilung in der Folgeperiode und die Verteilungswirkungen der zusätzlich zu beachtenden Zinssteuern berücksichtigt werden. Erst aus der Summierung dieser Faktoren kann sich ein Gesamtbild ergeben, da die intertemporalen Effekte keine Wirkung sui generis sind. Die oben dargestellte Diskussion zu den intertemporalen Verteilungwirkungen der Staatsverschuldung leidet darunter, daß jeweils nur Teilaspekte erfaßt werden.

So stellt der Wachstumsansatz lediglich auf Niveauentwicklungen ab, kann aber aufgrund der Vernachlässigung der Verteilungsstruktur keine verteilungspolitische Ausrichtung im engeren Sinne beanspruchen. Die mikroökonomischen Ansätze dagegen übergehen das Geschehen der Schuldaufnahmephase völlig, was auch bei neoklassischer Analyse die Vernachlässigung verteilungsrelevanter Sachverhalte bedeutet, und können bei Interpretation der Verzinsungsphase nur Ansatzpunkte für eine Verteilungsanalyse liefern.

Faßt man das Problem der intertemporalen Verteilung in kreislauftheoretischer Sicht zusammen, so ist für den Normalfall festzuhalten, daß sich die Verteilungsänderungen ausschließlich in der Schuldaufnahmephase ergeben. Werden dagegen die privaten Investitionen im Umfange der staatlichen Schuldaufnahme zurückgedrängt, so sind keine Auswirkungen auf die Verteilungssituation dieser Periode festzustellen, auch die strukturelle Verteilung der Folgeperiode wird konstant bleiben, wenn sich diese konstante Verteilung auch auf einem geringeren Niveau einspielen wird. Lediglich insoweit ergäbe sich eine "Lastverschiebung", bei näherer Interpretation wird aber die Fragwürdigkeit dieses Lastbegriffs deutlich. Alimentieren nämlich im Normalfall des keynesianischen Wirkungsmechanismus ausschließlich die Arbeitnehmer in der ersten Periode die staatlichen Güteransprüche, so würden bei klassischen Wirkungsmechanis men und konstanten Verteilungsquoten in den Folgeperioden beide Verteilungsgruppen belastet, wobei für beide Gruppen ein absolut geringeres Einkommensniveau gegeben wäre. Oben wurde schon auf die Problematik einer Bewertung dieses trade offs verwiesen, auf keinen Fall kann hier von Lastverschiebung gesprochen werden. Das Aufzeigen struktureller und absoluter Verteilungseffekte vermag dagegen zumindest eine Entscheidungshilfe zu geben. 


\section{E) EXKURS: INTERTEMPORALE VERTEILUNGSEFFEKTE BEI UNTERBE- SCHÄFTIGUNG ALS AUSGANGSSITUATION - LASTVERSCHIE BUNGSPARADOX}

Wenn unserem Inzidenzkonzept auch die Annahme der Vollbeschäftigung zugrunde liegt, sollen hier noch einige Überlegungen zu intertemporalen Verteilungseffekten für eine andere konjunkturelle Ausgangssituation angeschlossen werden. Oben wurde das Sozialprodukt als das Verteilungspotential beschrieben, an das (unter der Annahme der geschlossenen Wirtschaft) die drei Sektoren private Haushalte, Unternehmen und öffentliche Haushalte ihre Ansprüche richten.

Geht man davon aus, daß die Ansprüche des Staats an dieses Gütervolumen[274] zumindest konstant bleiben oder im Zeitablauf tendenziell zunehmen werden, so sieht sich der Staat je nach konjunktureller Ausgangssituation vor zwei grundlegend verschiedene Situationen gestellt. In Phasen der Hochkonjunktur muß auch die öffentliche Hand mit den Ansprüchen der anderen Sektoren um das gegebene Gütervolumen konkurrieren, wobel je nach gewählter Finanzierungsart die beiden anderen Gruppen gütermäßig belastet werden.

In Phasen der Unterauslastung der gesamtwirtschaftlichen Kapazitäten besteht dieses Konkurrenzverhältnis dagegen nicht. Das Verteilungspotential wird, bildhaft gesprochen, nicht einmal voll ausgeschöpft, ist doch eine, wenn nicht sogar die Hauptursache für eine solche konjunkturelle Situation in der zu geringen gesamtwirtschaftlichen Nachfrage zu sehen. Bekanntermaßen gehört es zu den konjunkturpolitischen Aufgaben des Staates, dieses gesamtwirtschaftliche Nachfragedefizit abzubauen.

Für eine konjunkturpolitisch eingesetzte Ausgabenpolitik empfiehlt sich eine solche Finanzierungsweise, die nicht an anderer Stelle kontraktiv wirkt. Mit einer Kreditnahme steht dem Staat ein Finanzierungsinstrument zur Verfuigung, das ihm bei entsprechender Handhabung die Möglichkeit bietet[275], ohne Verdrängungseffekte Anteile am Sozialprodukt an sich zu ziehen, um die er in Phasen der Hochkonjunktur mit anderen Sektoren wetteifern müßte[276]. Mit einer solchen Güterbeanspruchung führt der Staat darüber hinaus aufgrund der multiplikativen Effekte zusätzlicher Nachfrage das gesamte Verteilungspotential auf ein höheres Niveau. Da in einer solchen Situation das Primat der Verteilungspolitik bei der Niveaubetrachtung liegt, soll hier nicht auf die Entwicklung der strukturellen Verteilung eingegangen werden. Diese Zusammenhänge lassen sich nun in Verbindung mit den Problemen der intertemporalen Verteilung sehen.

Jedes Nicht-Herausführen aus einer Situation der Unterbeschäftigung bedeutet einen endgültigen Verlust an potentieller Verteilungsmasse, die durch die gesamtwirtschaftlich vorhandenen Kapazitäten vorgegeben ist. Auch bei einer solchen Ausgangssituation, bei der zunächst von einer Lastverschiebung nicht die Rede sein kann, da der Verlust an Verteilungspotential die augenblickliche Periode betrifft, gilt es, eine intertemporale Komponente zu berücksichtigen. 
Je nach sektoraler Konjunktursituation werden die Konsum- und Investitionsgüterindustrie in unterschiedlichem Maße betroffen, woraus sich analog zu oben intertemporale Effekte ableiten lassen[277].

Solche Situationen sind für ein marktwirtschaftliches System nicht vermeidbar, wenn auch aufgrund der staatlichen Einwirkungsmöglichkeiten steuerbar. Das Eingreifen des Staates ist aber neben dieser allgemeinen konjunkturpolitischen (und damit verteilungsniveaupolitischen) Begründung noch aus einem weiteren, die Sekundärverteilung betreffenden Aspekt von verteilungspolitischem Interesse.

Neben der Vermittlung konjunktureller Impulse kann der Staat zugleich einen haushaltspolitisch bedeutsamen Schritt tun. Geht man davon aus, daß der Staat angesichts einer Aufgabenfülle unter der Knappheit der ihm zur Verfügung stehenden Mittel leidet, so kann er in Zeiten der Unterbeschäftigung auf ohnehin brachliegende Ressourcen zurückgreifen. Sieht man nun die staatliche Ausgabenentscheidung als gegeben an (d.h., daß bestimmte Vorhaben in jedem Fall realisiert werden müssen), so bedeutet diese Realisierung in Rezessionsphasen, daß c.p. eine größere Gütermenge für den privaten Bereich in den Folgeperioden zur Verfügung steht. Läßt der Staat dagegen diese Möglichkeiten ungenutzt[278], so wird dadurch zum einen das Niveau des Verteilungspotentials unter dem möglichen Niveau gehalten, zum anderen der strukturelle Verteilungskonflikt in spätere Perioden transferiert.

Beansprucht der Staat also nicht subsidiär die Gütermengen, die quasi "frei" zur Verfügung stehen, wird er diesen strukturellen Verteilungskonflikt, der auf die Dichotomie zwischen privatem und öffentlichem Sektor abstellt, in die Zukunft verlegen. Damit ergibt sich eine Lastverschiebung, die diametral zum üblichen Ansatz steht, nämlich auf die Unterlassung der Kreditnahme des Staates zurückzuführen ist. Der traditionelle Ansatz untersucht die Möglichkeiten, wie die Belastung durch bestimmte Ausgaben des Staates auf spätere Perioden verlagert werden kann. Oben kamen wir zum Ergebnis, daß eben dies nicht wahrscheinlich sei. Nun konstatieren wir eine Belastung, wenn staatliche Aufgaben nicht in bestimmten Perioden erfüllt werden. Die Möglichkeit zur Lastverschiebung, d.h. einer im Zeitablauf variablen Verteilung zwischen öffentlichem und privatem Sektor beruht darauf, daß die öffentliche Hand aus übergeordneten Gesichtspunkten heraus ihre Ansprüche in den einzelnen Perioden variabel gestalten kann, ohne dabei in längerfristiger Sicht Verteilungs nachteile hinnehmen zu müssen. Es handelt sich bei dieser Betrachtung von "ausgelassenen Möglichkeiten" als Last wohl um eine recht extensive Interpretation, der aber doch eine verteilungspolitische Bedeutung zukommt. 


\section{WIRKUNGEN AUF DIE VERMÖGENSVERTEILUNG}

\section{A) GRUNDLEGENDE ZUSAMMENH ÄNGE}

1. Vermögensvertellung als verteilungspolitisches problem

Nachdem in den vorhergehenden Kapiteln den verschiedenen Wirkungen staatlicher Verschuldung auf die Einkommensvertellung nachgegangen wurde, soll nun (zur Abrundung) die Bedeutung der staatlichen Verschuldung für die Vermögenspolitik aufgezeigt werden. Die traditionelle Vermögenspolitik, die eine wie auch immer definierte gleichmäßigere Vermögensverteilung anstrebt, hat die Vermögensneubildung als wesentlichen Aktionsparameter im Auge. Entsprechend haben die Maßnahmen, die zur Veränderung einer als unbefriedigend empfundenen Verteilung des privaten Vermögens führen sollen, bei der Vermögensneubildung, d.h. an der Ersparnisbildung, anzusetzen. Unmittelbare Eingriffe in die Vermögensbestände zu Zwecken der Umverteilung bleiben iblicherwelse als systeminkonform außer Betracht.

Daneben hat in jüngster Zeit aufgrund steigender Aktualität ein neuer Aspekt an Bedeutung gewonnen. Verteilungsänderungen am bestehenden Vermögen entstehen nämlich auch durch unterschiedliche Wertentwicklungen einzelner Vermögensarten. Diese Effekte sind vor allem dann von verteilungspolitischem Interesse, wenn sich schichtenspezifische Besonderheiten bel den individuellen Vermögensstrukturen feststellen lassen[279]. Insbesondere Engels und Mitautoren haben auf den bedeutenden Umfang dieser Wertänderungen hingewiesen, die quantitativ die Dimensionen der Vermögensneubildung einer Periode erreichen und überschreiten können[280].

2. Vermögenspolitische Begrüdung einer staatlichen Schuldaufnahme

Jede staatliche Schuldaufnahme ist auch von vermögenspolitischer Relevanz, da sie ein Element der privaten Vermögensbildung darstellt. So wird dieses Finanzierungsinstrument auch von etlichen Autoren zu instrumentalem Einsatz vorgeschlagen. Dies kann - wie bel Willgerodt und Bartel[281] - auf ein primär ordnungspolitisch begründetes Mißtrauen gegenuber einer zu hohen staatlichen Vermögensbildung zurückzuführen sein, wobel diese Autoren gleichzeitig von einem staatlichen Nachholbedarf an Infrastrukturinvestitionen ausgehen. Insofern steht bei diesen Vorschlägen die Relation von staatlichem zu privatem Vermögen zur Diskussion. Der Problemkreis der strukturellen Ver- 
teilung des privaten Vermögens klingt eher beiläufig mit der Propagierung der breiten Streuung solcher Papiere an.

Haller dagegen argumentiert im Zuge einer neuen Stagnationsthese, die davon ausgeht, daß aufgrund einer strukturell bedingten Abschwächung der privaten Investitionsgüternachfrage bei gleichzeitig wachsendem Angebot an Ersparnissen der Staat einspringen müsse, um diese andernfalls deflatorisch wirkenden Beträge zu absorbieren[282].

Im folgenden soll deshalb unter Rückgriff auf die oben eingeführten makroökonomischen Wirkungsmechanismen die staatliche Verschuldung auf ihre Eignung als vermögenspolitisches Instrument untersucht werden. Dabei gilt es sowohl den Aspekt der Vermögensneubildung als auch den der Wertänderungen zu berücksichtigen. Die staatliche Neuverschuldung kann nämlich, wie oben gezeigt wurde, Mitverursacher von Umbewertungsvorgängen sein, stellt aber andererseits in Form der schon bestehenden Staatsschulden auch eine der von solchen Preisniveaueffekten negativ betroffenen Vermögensarten dar.

Die erwähnten Wirkungsmechanismen sind deswegen von Belang, weil mit ihnen unterschiedliche Wirkungen auf die Gesamtvermögensbildung einer Periode verbunden sind. Limitieren im klassischen System die freiwilligen Ersparnisse einer Periode die privaten Investitionen sowie die staatliche Verschuldung und damit die gesamtwirtschaftliche bzw. private Vermögensbildung, so bestimmen im keynesianischen System - bei Vorliegen der entsprechenden monetären Voraussetzungen - diese autonomen Nachfragebestandteile den Umfang der gesamtwirtschaftlichen Vermögensbildung. Dem bisherigen Vorgehen entsprechend stehen auch hier die Wirkungen für die Gruppe der Unternehmer und der Arbeitnehmer im Vordergrund, eine vermögenspolitische Beurteilung staatlicher Schuldaufnahme in einer Vollbeschäftigungssituation muß sich daran orientieren, wie die private Vermögensverteilungsstruktur beeinflußt wird. Die Zunahme dieses Aggregats allein kann kein hinlängliches Kriterium sein.

\section{B) WIRKUNGSANALYSE}

1. Auswirkungen einer staatlichen Neuverschuldung auf die Vermögensvertellung

a) Klassischer Fall

Dem Aspekt der zusätzlichen Vermögensbildung kommt im klassischen System nur dann Bedeutung zu, wenn mit einer hohen Zinselastizität der Ersparnis bildung zu rechnen ist, da nur im Umfang der zusätzlichen freiwilligen Ersparnis das Vermögensbildungsniveau zunimmt. Dies wurde oben als unwahrscheinlich charakterisiert, so daß man wohl von der Quasi-Konstanz der privaten Vermögensbildung aus freiwilligem Sparen ausgehen kann. Da mit dem Tatbestand der staatlichen Verschuldung keine Änderung bezüglich der Spar- 
fähigkeit verbunden ist, sind auch von daher keine strukturellen Effekte bezüglich der personellen Vermögensverteilung zu erwarten.

Der klassische Fall ist aber mit Zinssatzsteigerungen verbunden, durch die die Verdrängung privater Investitionen bewirkt werden soll. Auch davon geht ein Einfluß auf die Vermögensverteilung aus, da jede Zinssatzänderung eine Umbewertung der Bestände zur Folge hat[283]. Dies gilt für alle Vermögensarten einschließlich Realkapital, da bei der Ertragswertbestimmung der Zins in Form des Diskontierungsfaktors einen bedeutenden Einfluß ausübt[284]. Noch unmittelbarer werden diese Effekte bei börsenfähigen, festverzinslichen Wertpapieren sichtbar, bei denen durch Kursverluste auf Marktzinserhöhungen hin die Effektivverzinsung an das neue Zinsdatum angepaßt wird. Diese $\mathrm{Be}-$ wertungsänderungen sind aber nur für Wirtschaftssubjekte von Belang, die sich vor Ende der Laufzeit der Papiere von diesen trennen (müssen). Werden diese Bewertungsverluste nicht realisiert und diese zum vereinbarten Nominalkurs zurückgenommen, spielt dieser Aspekt in vermögenspolitischer Sicht keine entscheidende Rolle[285][286], stellt er doch nur das spezifische Risiko einer bestimmten Anlageform dar.

\section{b) Keynesianischer Fall}

Dieser Wirkungsmechanismus ist in seiner idealtypischen Form gerade dadurch gekennzeichnet, daß die durch zusätzliche staatliche Kreditnachfrage notwendig werdende Vermögensbildung durch Änderungen der Einkommens verteilung realisiert wird. Auch der Ort bzw. die Gruppe ist eindeutig lokalisierbar, wo sich diese zusätzliche Vermögensbildung niederschlägt. Geht man nämlich von einer Konstanz des Sparverhaltens aus, was oben als plausible Annahme charakterisiert wurde, so werden diesem Mechanismus zufolge solange Einkommensumschichtungen zugunsten der Gruppe mit der höheren Sparneigung erzwungen, bis der Umfang der notwendig gewordenen Ersparnisbildung erreicht ist. Die oben beschriebenen Änderungen der Einkommensverteilung schlagen sich zum großen Teil in Vermögensbildung nieder. Diese beiden Wirkungen sind kausal verknüpft, im Umfange aber nicht deckungsgleich, da nicht alle zusätzlichen Einkommen vermögenswirksam werden[287]. Da die Notwendigkeit zusätzlicher Vermögensbildung die Ursache für die Verschiebung der Einkommensverteilung ist, kann das vermögenspolitische Urteil nicht positiver als das bezüglich der Einkommensverteilungseffekte sein. Es ist $z u$ betonen, daß diese Wirkungen völlig unabhängig davon eintreten werden, wer die staatlichen Papiere kauft. Sowohl die Effekte bezüglich der Einkommens- als auch der Vermögensverteilung ergeben sich im Rahmen des allgemeinen Einkommens- und Vermögensbildungsprozesses. Individuelle Antageentscheidungen sind für diese Zusammenhänge ohne Belang, sofern nicht Verhaltensänderungen zu konstatieren sind. Auch diese Aussage mündet in die Empfehlungen allgemeiner Vermögenspolitik, daß Änderungen der Vermögensverteilung nur über geänderte Sparverhalten erzielbar sind. So ist auch die vermögenspolitische Beurteilung Beispiel dafür, daß aus 
partialanalytischen Überlegungen (etwa bezüglich der Anlageform) noch keine Schlüsse auf die effektiven Wirkungen gezogen werden können. Da die zusätzliche Vermögensbildung durch Realeinkommensverschiebungen erzwungen wird, die ihrerseits iber Preisniveaueffekte erzielt werden, treten auch hier Umbewertungen des bestehenden Vermögens zutage. Im Gegensatz zum oben geschilderten Fall einer Umbewertung sind hier aber je nach Vermögensart unterschiedliche Auswirkungen $\mathrm{zu}$ beachten.

Dieser Problemkreis ist in der allgemeinen Diskussion der Verteilungseffekte einer Inflation als Gläubiger-Schuldner-Hypothese[288] bekannt geworden. Da die staatliche Kreditaufnahme Verursacher inflationärer Impulse sein kann, soll auf diese Hypothese und thre mögliche Aussagefähigkeit für die strukturelle Vermögensverteilung näher eingegangen werden.

\section{Gläubiger-Schuldner-Hypothese}

Alle Arten von Nominalvermögen, worunter fixierte Ansprüche auf eine bestimmte nominale Summe $\mathrm{zu}$ verstehen sind, unterscheiden sich von Realvermögen dadurch, daß bei Geldwertminderungen, d.h. dem Vorliegen inflationärer Impulse, ihr Realwert im Zeitablauf sinkt. Betrachtet man die Wertänderungen am aggregierten Geldvermögen einer Periode, so ist deren Umfang abhängig vom Gesamtvolumen dieser Vermögensart und dem Ausmaß der Preissteigerungen. Ein diesen Verlusten entgegenwirkender Faktor kann eine Steigerung des Zinssatzes sein, da sich dadurch möglicherweise ein zumindest anteiliger Inflationsausgleich ergibt[289]. Die Einschätzung dieser Rolle des Zinssatzes ist recht uneinheitlich; sie reicht von der vorsichtigen Äußerung Zierckes, die Preisniveausteigerungen gäben lediglich die Obergrenze der Verluste an[290], bis zur völligen Ablehnung eines Inflationsausgleichs durch Pfleiderer[291]. Bei gesamtwirtschaftlicher Betrachtung steht allerdings jedem geschädigten Gläubiger ein entsprechend begünstigter Schuldner gegenuiber, so daß sich in der von uns unterstellten geschlosssenen Volkswirtschaft diese Gewinne und Verluste gegenseitig aufheben[292]. Daher ist es für eine verteilungspolitische Aussage unerläßlich, die Nettopositionen einzelner verteilungsrelevanter Gruppen zu untersuchen[293].

Zur Klärung dieser Frage müssen die Gruppenanteile am gesamten Nominalvermögen bekannt sein, da alle Besitzer dieser Vermögensart von den oben beschriebenen Effekten betroffen werden. Neben den Anteilen am Nominalvermögen ist aber auch die jeweilige Schuldnerposition $\mathrm{zu}$ berücksichtigen, da erst die Nettoeffekte vertellungspolitisch entscheidend sind.

Ein für diese Fragestellung optimal aufbereitetes Material[294] findet sich bei Engels/Sablotny/Zickler[295], die bei ihrer Darstellung der sozioökonomischen Struktur die auch in dieser Arbeit zugrundegelegte Differenzlerung in Selbständige und Unselbständige vornehmen. Im folgenden seien die typischen Portfoliostrukturen dieser belden Haushaltsgruppen tabellarisch nach Engels wiedergegeben[296]: 
Durchschnittsportefeuilles von Selbständigen- und Unselbständigenhaushalten (jeweils in \%)

Selbständige:

$\begin{array}{ccccr}\text { Jahr } & \begin{array}{l}\text { Geldver }- \\ \text { mögen }\end{array} & \begin{array}{l}\text { Wohn- } \\ \text { eigentum }\end{array} & \begin{array}{l}\text { Gewerbliches } \\ \text { Betriebs vermögen }\end{array} & \begin{array}{l}\text { Landwirtsc } \\ \text { Betriebsve }\end{array} \\ 1950 & 3,5 & 20,1 & 23,9 & 52,5 \\ 1955 & 7,0 & 16,0 & 30,3 & 46,7 \\ 1960 & 8,4 & 15,7 & 38,8 & 37,1 \\ 1965 & 9,5 & 16,5 & 37,5 & 36,1 \\ 1970 & 11,1 & 18,6 & 42,2 & 28,1\end{array}$

Unselbständige:

$\begin{array}{rrrrr}1950 & 16,9 & 52,4 & 8,4 & 22,3 \\ 1955 & 27,0 & 44,9 & 11,0 & 17,1 \\ 1960 & 30,4 & 43,1 & 15,2 & 11,3 \\ 1965 & 33,8 & 46,7 & 10,3 & 9,2 \\ 1970 & 37,6 & 46,8 & 9,8 & 5,8\end{array}$

Wenn wir auch im Rahmen unserer Fragestellung nicht auf die Entwicklung der Portfoliostrukturen abstellen wollen, zeigt sich doch gerade beim Aggregat Geldvermögen[297] eine zunehmende Bedeutung für die Haushalte der Unselbständigen. Bereits aus diesen Relationen ergibt sich die tendenzielle Inflationsgefährdung der Haushalte der Unselbständigen[298], doch muß darüber hinaus auch auf die Schuldnerposition, die für die hier zugrundegelegte Hypothese die Gegenposition darstellt, eingegangen werden. Auch diese Gegenüberstellung findet sich in entsprechend sozioökonomischer Differenzierung bei Engels, wobei die Problematik dieser Daten darin zu sehen ist, daß jeweils beide Positionen getrennt als Nettogröße vorliegen. In der folgenden Tabelle, die lediglich die letzten Jahre berücksichtigt, zeigt sich eindeutig, daß die Gläubigerposition der Unselbständigenhaushalte bei weitem deren Schuldnerposition übersteigt.

Selbständigenhaushalte

$\begin{array}{lcll}\text { Jahr } & \begin{array}{l}\text { Nettoforderungs - } \\ \text { position } \\ \text { (in Mrd. DM) }\end{array} & \begin{array}{l}\text { Zurechenbare } \\ \text { Nettoverbindlich- } \\ \text { keiten (in Mrd.DM) }\end{array} & \begin{array}{l}\text { Gesamt- } \\ \text { position } \\ \text { (in Mrd. DM) }\end{array} \\ 1965 & 70,7 & -109,2 & -38,5 \\ 1966 & 80,3 & -119,0 & -38,7 \\ 1967 & 86,8 & -123,5 & -36,7 \\ 1968 & 94,5 & -134,8 & -40,3 \\ 1969 & 104,8 & -159,3 & -54,5 \\ 1970 & 116,4 & -178,8 & -62,2\end{array}$


Unselbständigenhaushalte

$\begin{array}{lccc}\text { Jahr } & \begin{array}{l}\text { Nettoforderungs - } \\ \text { position } \\ \text { (in Mrd. DM) }\end{array} & \begin{array}{l}\text { Zurechenbare } \\ \text { Nettoverbindlich- } \\ \text { keiten (in Mrd. DM) }\end{array} & \begin{array}{l}\text { Gesamt- } \\ \text { position } \\ \text { (in Mrd. DM) }\end{array} \\ 1965 & 142,4 & -83,6 & 58,8 \\ 1966 & 164,1 & -93,4 & 70,7 \\ 1967 & 185,8 & -102,1 & 83,7 \\ 1968 & 213,3 & -111,9 & 101,4 \\ 1969 & 243,2 & -125,4 & 117,8 \\ 1970 & 278,6 & -136,4 & 142,2\end{array}$

"Diese strukturellen Unterschiede führen dazu, daß sich inflatorische Tendenzen stark auf die Gesamtvermögenspositionen der Selbständigen - und Unselbständigenhaushalte auswirken und damit auch auf die Verteilung zwischen den sozialen Gruppen. Die Disparitäten zwischen den sozialen Gruppen können sich deshalb tendenziell stärker behaupten, weil die Gesamtvermögens position der Unselbständigenhaushalte strukturell inflationsempfindlich ist"[299].

Wie auch schon bei den Aussagen zur Einkommensverteilung ist bei dieser hochaggregierten Betrachtung auf die Gefahren der Durchschnittsbildung zu verweisen. Dies trifft in noch stärkerem Umfang auf Probleme der Vermögens verteilung $z u$, da die Verteilung der Vermögensbestände auch innerhalb der sozioökonomischen Gruppen extrem schief ist[300]. So relativieren Engels und Mitautoren auch diese Durchschnittswerte, indem sie darauf verweisen, daß etwa landwirtschaftliches oder gewerbliches Vermögen, aber auch Grundvermögen nur bei einem geringen Teil der Unselbständigenhaushalte zu finden ist. "Der Tendenz nach kann man sagen, daß auch innerhalb der sozialen Gruppen der Anteil des Geldvermögens um so größer ist, je weniger Vermögen insgesamt vorhanden ist. Insofern verschiebt die Inflation nicht nur die Vermögenspositionen zugunsten der Selbständigen, sondern ganz allgemein zugunsten der wohlhabenderen Haushalte. In der sehr groben Aggregation auf die Gruppen 'Selbständige' und 'Unselbständige' geht ein wesentlicher Teil der Vermögensverteilungswirkungen der Inflation verloren"[301]. So kommt Wicke zum Schluß, daß die Inflationsschädigung primär durch das Vorhandenbzw. Nichtvorhandensein von Grund-und Hausbesitz bestimmt wird, nicht aber durch die Zugehörigkeit zu einer bestimmten sozialen Schicht[302].

Wenn mit diesen Ergebnissen auch keine endgültige Beurteilung möglich scheint, ergibt sich doch das tendenzielle Ergebnis, daß insbesondere die Kleineinkommensbezieher in der Klasse der Unselbständigen ohne Haus- und Grundbesitz aufgrund der Dominanz der Anlage in Geldvermögen[303] von diesen Umbewertungen betroffen werden, in ihrem Portefeuille sich also kein Aggregat befindet, das eine gegenläufige Wertentwicklung erfährt[304]. 


\section{Schlußfolgerung}

Setzt man die staatliche Verschuldung in einer Vollbeschäftigungssituation zu vermögenspolitischen Zwecken ein, so besteht dadurch eine dreifache Gefahr, verteilungspolitischen Zielsetzungen zuwiderzuhandeln, solange nicht ein völliges Crowding-out eintritt, was aber als unwahrscheinlich anzunehmen ist. Zunächst können negative Verteilungseffekte für die Gruppe der Arbeitnehmer in bezug auf die Einkommensverteilung auftreten, denen die Kreislaufgewinne bei den Unternehmern entsprechen, woraus dann die gesamtwirtschaftlich notwendig werdende zusätzliche private Vermögensbildung resultiert. Damit liegt auch bereits die vermögenspolitische Beurteilung auf der Hand, wird dieses Vermögen doch aus den umverteilten Einkommen bei den Unternehmern gebildet.

Darüber hinaus gehen aber auch verteilungspolitisch bedenkliche Rückwirkungen auf die Vermögensbestände aus, die insbesondere untere Einkommens schichten in der Gruppe der Arbeitnehmer aufgrund der dort gegebenen Einseitigkeit der Vermögensanlagen, die keinen Inflationsausgleich zuläßt, treffen. Unter diesen Voraussetzungen fällt das vermögenspolitische Urteil eindeutig negativ aus. Es ist hervorzuheben, daß diese Beurteilung vollkommen unabhängig davon ist, wer die Staatspapiere zeichnet.

Geht man im Gegensatz hierzu von substitutiven Beziehungen zwischen den privaten Investitionen und der staatlichen Neuverschuldung aus, nimmt also an, daß die private Vermögensbildung konstant bleibt, so kann auch in diesem Falle das vermögenspolitische Ziel nicht erreicht werden. Allerdings sind dann die oben aufgezeigten negativen Begleiterscheinungen nicht zu erwarten, auch die Einkommensverteilung wird konstant bleiben.

Eine Veränderung der Vermögensverteilung zugunsten der Arbeitnehmer ist nur dann möglich, wenn von dieser Gruppe zusätzliche Vermögensbildung be trieben wird, die uber reine Anlagesubstitution hinausgehen muß. 



\section{Siebtes Kapitel}

\section{Z USAMMENFASSUNG DER ERGEBNISSE}

Vorrangiges Untersuchungsziel unserer Überlegungen war, die mit einer staatlichen Kreditaufnahme verbundenen Vertellungseffekte interpersoneller und intertemporaler Art in einem Gesamtzusammenhang aufzuzeigen. Mit diesem Anspruch eng verbunden ist die Wahl des kreislauftheoretischen Erklärungsansatzes der Verteilung, da eine Erfassung der Verteilungswirkungen dieses staatlichen Finanzierungsinstruments ohne Berücksichtigung der Kreis laufzusammenhänge nicht möglich scheint. Diesem Ansatz entsprechend standen die makroökonomischen Quoten der institutionellen Verteilung als Indikatoren im Vordergrund. Gleichzeitig sollte die Untersuchung aber auch einer formalen und inhaltlichen Auseinandersetzung mit den Aussagen der klassischen Schuldtheorie dienen, diesem Zweck entspricht das auf eine Situation der Vollbeschäftigung ausgerichtete Inzidenzkonzept.

Als entscheidendes materielles Ergebnis muß festgehalten werden, daß die strukturellen Verteilungseffekte unmittelbar in der Periode der Kreditaufnahme in Erscheinung treten. Dabei wird sich unter realistischen Annahmen immer eine Verbesserung der Verteilungssituation der Unternehmer ergeben. Der Umfang dieser Verteilungsänderungen ist zum einen von dem über geldpolitische Maßnahmen beeinflußbaren Crowding-out, zum anderen von der auf die Kreislaufprofite bezogenen Sparneigung abhängig, die die möglichen multiplikativen Effekte bestimmt.

Nur bei aktiven geldpolitischen Eingriffen wird überhaupt ein Crowding-out eintreten, da eine nachfragewirksame staatliche Verschuldung insgesamt stimulierend auf die private Investitionsneigung wirkt. Meist ist es aber bei den gegebenen, institutionellen Voraussetzungen nahezu unmöglich, ein volles Crowding-out herbeizuführen. Keinesfalls kann aber der von der klassischen Theorie unterstellte, gewissermaßen automatische Wirkungsmechanis mus Gültigkeit beanspruchen.

Die Verteilungseffekte einer Schuldaufnahme haben den Charakter eines einmali gen, nur in dieser Periode wirkenden Impulses, die Verteilung der Folgeperiode wird - von der Verzinsung sei noch abstrahiert - nicht mehr berührt.

Nicht minder wichtig für das Ergebnis des punktuellen zeitlichen Auftretens der Verteilungswirkungen in der Phase der Schuldaufnahme ist der Umstand, daß von der Verzinsung keine entscheidenden Verteilungseffekte ausgehen. Die Umverteilung nominaler Beträge (und nichts anderes stellt der SteuerZins-Kreislauf dar) wird solange keine Verteilungsänderungen zwischen den beiden großen Einkommensgruppen bewirken, als damit nicht auch Verhaltens- 
änderungen verbunden sind. Anders formuliert bedeutet dies, daß bei gegebener Verwendungsstruktur des Sozialprodukts und von der Einkommensart unabhängigen, an Realgrößen ausgerichteten Verhaltensparametern der beiden Verteilungsgruppen eine Veränderung der institutionellen Verteilung nicht möglich ist.

Entsprechend obigen Ergebnissen können auch die intertemporalen Verteilungsaspekte keine bedeutende Rolle spielen. Geht man von einer gütermäßigen Betrachtung aus, so ist bei Vollbeschäftigung in der Periode der Schuldaufnahme das gesamtwirtschaftliche Verteilungspotential gegeben und durch eine staatliche Kreditnahme nicht vermehrbar. Wohl aber kann die Verwendungsstruktur des Sozialprodukts beeinflußt werden, was dann Auswirkungen auf die Verteilungsniveaus künftiger Perioden hat, wenn die Privatinvestitionen durch eine staatliche Kreditnahme zurückgedrängt werden. Entscheidend sind also diese Beziehungen zwischen möglichen Verwendungsstrukturen und zukünftigen Verteilungspotentialen. So können lediglich im Falle eines Crowding-out die zukünftigen Verteilungsniveaus im Vergleich zu einer Situation ohne staatliche Schuldaufnahme tangiert werden, sofern der Wachstumsbeitrag der staatlichen Projekte unter dem der verdrängten Privatinvestitionen liegt. Die Problematik der intertemporalen Verteilung mündet dann in die Forderung nach rationaler staatlicher Ausgabenpolitik.

Die Analyse sollte auch den entscheidenden Ansatzpunkt für die verteilungspolitische Beurteilung einer staatlichen Schuldaufnahme zeigen. Kriterium kann nur die gesamtwirtschaftliche Verwendungsstruktur und ihre Entwicklung in der betreffenden Periode sein. Diese Betrachtung muß sich also an Stromgrößen orientieren, die Berücksichtigung irgendwelcher auf die Bestandsgröße der staatlichen Schuld bezogenen Kennziffern (etwa die häufig herangezogene Pro-KopfVerschuldung) ist ohne Aussagewert. Allerdings ist bei der verteilungspolitischen Analyse auch die Nettoschuldaufnahme der Vorperiode zu berücksichtigen, ihrem relativen Anteil am Sozialprodukt entsprechend kann in dieser Periode eine Neuverschuldung erfolgen, ohne daß sich die obengenannten Verteilungseffekte einstellen.

Neben der Ermittlung der effektiven Inzidenz sollte aber gleichzeitig die Schwäche der klassischen Schuldtheorie in zweifacher Hinsicht deutlich geworden sein. Zum einen handelt es sich bei ihr um keine eindeutig auf ein verteilungstheoretisches Konzept bezogene Betrachtung, sondern eher um Ansatzpunkte der Analyse, zum anderen kann aber auch bei verteilungstheoretischer, d.h. für die klassische Schuldtheorie grenzproduktivitätstheoretischer, Fundierung dieser Ansatz in kurzfristiger Sicht keine zwingenden Ergebnisse liefern, da er wesentliche verteilungsrelevante Phänomen überhaupt nicht zu erfassen vermag. Unabhängig von der zugrundegelegten verteilungstheoretischen Erklärung muß die formale Behandlung des Phänomens der Staatsverschuldung identisch sein. Sowohl beim kreislauftheoretischen als auch beim grenzproduktivitätstheoretischen Ansatz muß die staatliche Kreditnahme als Teil der gesamtwirtschaftlichen Vermögensbildung verstanden, den jeweiligen ansatzspezifischen Wirkungsmechanismen entsprechend die Wirkungen auf die Einkommensverteilung 
verfolgt werden[305]. Nur so ist es möglich, die Trugschlüsse der traditionellen Behandlung der staatlichen Schuld $\mathrm{zu}$ vermeiden, die - nach Gandenberger die Inzidenz der Ersparnisbildung mit der der Staatsverschuldung verwechselte.

Würdigt man vor dem Hintergrund der kreislauftheoretischen Analyse die Aussagen der klassischen Schuldtheorie und ihres Inzidenzkonzepts, so treten nicht unerhebliche Widersprüche auf. Der in diesem Inzidenzkonzept als Normalfall und zwangsläufig eintretende Wirkungsmechanismus[306] kann unseren Überlegungen nach nur Sonderfallcharakter haben. Die von der neoklassischen Inzidenzkonzeption implizit unterstellten kompensatorischen geldpolitischen Eingriffe werden in vielen Fällen überhaupt nicht oder nur in beschränktem Maße möglich sein. Wenn die Forderungen dieses Konzepts aber nicht erfüllbar sind, können dessen Aussagen nicht mehr den Anspruch erheben, die Realität zu erklären, eine wirtschaftspolitische Nutzung der Aussagen dieses Konzepts wird unsinnig.

Indem alle von der staatlichen Verschuldung möglicherweise ausgehenden inflationären Effekte hinwegdefiniert werden, nimmt man einen willkürlichen Einschnitt in die Wirkungsanalyse vor. "All this is like arguing that if you let a boy shatter your front window with a stone, the stone and not the boy causes the damage"[307].

Die Möglichkeit einer Vereinfachung und Verabsolutierung dieser Inzidenzvorstellung im politischen Bereich ist aber nicht von der Hand zu weisen, womit offenkundig die Gefahr erheblicher Verstöße gegen verteilungspolitische Zielsetzungen verbunden ist.

Nicht zuletzt um dem vorzubeugen bzw. die Unhaltbarkeit des von klassischen Vorstellungen geprägten Inzidenzkonzeptes aufzuzeigen, wurden unsere Überlegungen innerhalb eines Konzeptes durchgeführt, das die inflationären Impulse mit erfaßt. Dabei soll die kreislauftheoretisch fundierte Inzidenzbetrachtung nicht irgendeine zusätzliche oder alternative Möglichkeit darstellen, sondern sie erhebt den Anspruch, der der vorliegenden Fragestellung adäquate analytische Ansatz zu sein.

Auch beim kreislauftheoretischen Ansatz stehen die möglichen Crowding-outEffekte im Mittelpunkt, jedoch wird nicht wie beim klassischen Ansatz von einer Eindimensionalität der Wirkungen ausgegangen. Mit dem Aufzeigen der als wahrscheinlich anzunehmenden Abläufe und ihrer Bestimmungsfaktoren ist auch die Möglichkeit gegeben, andere Situationen zu erfassen. Die hier aufgezeigte Inzidenz der Staatsverschuldung ist damit eine ungleich bessere Grundlage für wirtschaftspolitische Entscheidungen als die Aussagen der nur scheinexplikativen klassischen Inzidenzkonzeption. 

1 Vgl. H.Zimmermann: Der letzte "klassische" Deckungsgrundsatz, in: Finanzarchiv, NF Band 24, 1965, S.70ff.

2 Vgl. W.Dreißig: Zur Entwicklung der öffentlichen Finanzwirtschaft seit dem Jahr 1950, in: Deutsche Bundesbank (Hrsg.): Währung und Wirtschaft in Deutschland 1876-1975, Frankfurt 1976, S.730.

3 Vgl. J.Beyfuss: Gesamtwirtschaftliche Probleme der Staatsverschuldung. Beiträge zur Wirtschafts- und Sozialpolitik 33, Institut der deutschen Wirtschaft, Köln 1976, S. 7.

4 So F.Modigliani: Long-Run Implications of Alternative Fiscal Policies and the Burden of the National Debt, in: J.M.Ferguson (Hrsg.): Public Debt and Future Generations, Chapel Hill 1964, S.107 und G.Schmölders: Grenzen der Staatsverschuldung, in: Zeitschrift für das gesamte Kreditwesen, 20. Jg., 1967, S. 1069.

5 Es ist hier daran zu erinnern, daß die vor allem im angelsächsischen Sprachraum ausgetragene Kontroverse um die intertemporalen Effekte der Staatsverschuldung im Anschluß an die Thesen Buchanans erst 15 Jahre zurückliegt.

6 Vgl. N. Andel: Zur These von den unsozialen Verteilungswirkungen öffentlicher Schulden, in: Public Finance, Band 24, 1969, S.74. A. Oberhauser: Stabilitätspolitik bei steigender Staatsquote, Schriften der Kommission für wirtschaftlichen und sozialen Wandel Nr. 43, Göttingen 1975, S.43. G. Tolkemitt: Zur Theorie der langfristigen Wirkungen öffentlicher Verschuldung, Tübingen 1975, S. 34/35.

7 Siehe dazu den Hinweis bei Hansmeyer/Mackscheidt, daß bei einer Beurteilung der staatlichen Finanzierungsmöglichkeiten alternativer Ausgabensenkung, Steuererhöhung und staatlicher Verschuldung die letzte Alternative die stärkste Ablehnung fand. K. -H. Hansmeyer und K. Mackscheidt: Finanzpsychologie, in: F. Neumark (Hrsg.): Handbuch der Finanzwissenschaft, 3. Auflage, Tübingen 1976, Teillieferung 7-9, S. 579.

$8 \mathrm{Zu}$ den grundsätzlichen Schwierigkelten der Leglslative gegenüber finanzpolitischen Fragestellungen vgl. G.Schmölders: Finanz- und Steuerpsychologle, Reinbek 1970, S.151-167.

9 In diesem Zusammenhang ist einerseits auf den im deutschsprachigen Raum lange (und tellweise immer noch) vertretenen Transfer-Ansatz, andererseits an die durch Andel aufgeworfene Forderung nach Betrachtung der "relevanten Alternative" $z$ u verweisen. Beide Aspekte werden unten noch eingehender erörtert. 
10 So kommt Andel in der Besprechung eines Sammelbandes, der zentrale Beiträge zu dieser Diskussion enthält, zum Schluß, "daß man zu unterschiedlichen Ergebnissen kommen kann, wenn man andere Annahmen macht, die Zahl der erfaßten Aspekte variiert oder Begriffe unterschiedlich definiert." N. Andel: Öffentliche Schulden und Lastverteilung, in: Finanzarchiv, NF Band 25, 1966, S.338.

Wenn Andel dieser Diskussion auch positive Aspekte abgewinnt, ist mit diesem Zitat doch ein nicht unwesentlicher Sachverhalt umschrieben.

11 So bezeichnet etwa Mishan die Beteiligten an dieser Diskussion wenig schmeichelhaft als "burden-mongers". Lerner beklagt die "linguistic tricks" und "semantic playfulness". Vgl. E.J.Mishan: How to Make a Burden of the Public Debt, in: J.M.Ferguson (Hrsg.): Public Debt and Future Generations, Chapel Hill 1964, S.201 und A.P. Lerner: The Burden of Debt, in: J.M.Ferguson (Hrsg.): Public Debt and Future Generations, a.a.O., S. 93 und 95 .

12 Vgl. K. Meister: Das Problem der Last in der Theorie der öffentlichen Schuld, Diss. Erlangen Nürnberg 1967, S.8, FN 1).

13 Als Beispiel für eine solch ergebnisbezogene Argumentation kann etwa die Arbeit von Kiepe angeführt werden. K.Kiepe: Die Thesen James M. Buchanans zur Last der Staatsverschuldung, Diss. Münster 1970.

J.M.Buchanan und M.R.Flowers: The Public Finances, 4.Auflage, Homewood 1975, S.329.

15 Mit dem Begriff der klassischen Schuldtheorie sollen die Ansätze bzw. Aussagen zu den Wirkungen einer staatlichen Schuldaufnahme charakterisiert werden, die mehr oder weniger stillschweigend davon ausgehen, daß im Umfange der staatlichen Beanspruchung investierbarer Mittel die private Investitionstätigkeit zurückgedrängt werden müsse. Dahinter steht die neoklassische Vorstellung, daß die gesamtwirtschaftliche Ersparnisbildung einer Periode die gesamtwirtschaftliche Investitionstätigkeit sowie die kreditfinanzierten staatlichen Ansprüche an das Sozialprodukt limitiert.

Man denke dabei an Differenzierungen wie die nach realen oder monetären Aspekten oder nach mikro- und makroökonomischen Ansätzen. Vgl. K. -H. Hansmeyer: La dette publique comme moyen de reporter des charges sur les générations futures, in: Revue de Science Financière, Band 55, 1963, S. 546 .

17 Vgl. entsprechende Forderungen bei O.Gandenberger: Intertemporale Verteilungswirkungen der Staatsverschuldung, in: H.Haller $u$. W. Albers (Hrsg.): Probleme der Staatsverschuldung. Schriften des Vereins für Socialpolitik, N.F. Band 61, Berlin 1972, S.190/191.

Zur näheren Begründung sei hier auf den Abschnitt II.3. verwiesen. 
19 Es ist $z u$ betonen, daß die klassische Schuldtheorie nicht auf dem neoklassischen (grenzproduktivitätstheoretischen) Verteilungsansatz basiert, sondern bei ihren partialanalytischen Aussagen lediglich von neoklassischen Wirkungsmechanismen ausgeht.

20 In solcher Absolutheit läßt sich diese Aussage allerdings seit Erscheinen des Aufsatzes von Gandenberger nicht mehr aufrechterhalten. Vgl. O.Gandenberger: Öffentlicher Kredit und Einkommensverteilung, in: Finanzarchiv, NF Band 29, 1970, S.1ff.

21 Deshalb wird im Mittelpunkt dieses Kapitels V. die Auseinandersetzung mit einigen repräsentativen Autoren stehen.

22 Zur Vernachlässigung der verteilungspolitischen Berücksichtigung der Ausgabenseite vgl. R.L.Frey: Finanzpolitik und Verteilungsgerechtigkeit, in: Finanzarchiv, NF Band 31, 1972, S.3.

23 In Situationen unausgelasteter Kapazitäten ist dieser Zusammenhang nicht gegeben, da die Ansprüche eines Sektors nicht zu Lasten eines anderen gehen müssen.

24 Vgl. D. Pohmer: Die Staatsverschuldung als Instrument der Verteilungspolitik, in: H.Haller u. W. Albers (Hrsg.): Probleme der Staatsverschuldung, a.a.O., S.164. Siehe dazu allerdings Einschränkungen bei K. -H. Hansmeyer: Der öffentliche Kredit, 2. Auflage, Frankfurt 1970, S.9f und G.Schmölders: Finanzpolitik, 3. Auflage, 1970, S. 394.

25 Lediglich der Fall von Zwangsanleihen soll mit dieser Definition ausgeschlossen sein. Vgl. zu dieser Sonderform H.Haller: Zur Problematik der Kreditfinanzierung öffentlicher Ausgaben, in: Finanzarchiv, NF Band 19, 1958/59, S. 79-87 und D. Pohmer, a.a. O., S. 164-177.

26 Es sei an die Annahme der Vollbeschäftigung erinnert.

27 Vgl. A.Stobbe: Untersuchungen zur makroökonomischen Theorie der Einkommensverteilung, Tübingen 1962, S. 35 .

28 Vgl. J.Kromphardt: Kapitalbildung in Arbeitnehmerhand und Einkommensverteilung im Gleichgewicht, in: Zeitschrift für die gesamte Staatswissenschaft, Band 122, 1966, S.249, FN 1).

29 Vgl. H. -J.Krupp: "Personelle" und "funktionelle" Einkommensverteilung, in: Jahrbuicher für Nationalökonomie und Statistik, Band 180, 1967, S. 7. G.Blümle: Theorie der Einkommensverteilung, Berlin-Heidelberg-New York 1975, S.6.

30 Am Rande sei schon hier vermerkt, daß das Sparverhalten von der in jeder Gruppe dominanten Einkommensart abhängig ist.

31 Die staatliche Schuldaufnahme zeichnet sich gerade dadurch aus, daß in der ersten Periode nur die Primärverteilung tangiert wird. 
32 Daß die Beschränkung ausschließlich auf Zahlungsströme ein unvollständiges Bild staatlicher Verteilungsaktivität abgibt, ist selbstverständlich, doch soll dieser Aspekt hier nicht weiter verfolgt werden. Vgl. dazu K:D. Henke: Die Vertellung von Guitern und Diensten auf die verschiedenen Bevölkerungsschichten, Schriften der Kommission für wirtschaftlichen und sozialen Wandel 82 , Göttingen 1975, S. 6 ff.

33 Vgl. G.Eisner: Kaufkraftubertragungen durch öffentliche Finanzen, Winterthur 1956, S.6. U.Gruber: Einkommensvertellung. Wirtschaft und Gerechtigkeit, München/Wien 1974, S.111/112.

34 So weist Bombach darauf hin, daß sich im Wachstum auch bel Konstanz der Quoten die absoluten Abstände Im Umfange des Niveauwachstums verändern. Vgl. G.Bombach: Neue Dimensionen der Lehre von der Einkommensverteilung, Basler Universitätsreden, 66. Heft, Basel 1972, S.19/20.

35 Ein weiterer problematischer Fall ist der der Unterbeschäftigung, wo eine ausschließliche Quotenbetrachtung zu Fehlschluissen führen kann, da bei zunehmender Beschäftigung alle Gruppen Verteilungsvorteile in Niveaubetrachtung erlangen können, unabhängig von gleichzeitig möglichen Struktureffekten. Vgl. U.Schillert: Gewinne als Quelle der Vermögenspolitik? Die Belastbarkeit der Unternehmensgewinne durch vermögenspolitische Maßnahmen, Berlin 1976, S.42.

36 In dieser weiten Fassung findet sich der Inzidenzbegriff etwa bei H.Fecher: Inzidenzprobleme finanzpolitischer Mittel zur Vermögensumverteilung, in: Schriften des Vereins für Socialpolitik, W.Albers (Hrsg.): Öffentliche Finanzwirtschaft und Verteilung I, NF Band 75, I, Berlin 1974, S.123. S.a. O.Gandenberger: Öffentlicher Kredit und Einkommensvertellung, a.a.O., S.4, FN 1).

37 Vgl. H. -C.Recktenwald: Steuerüberwälzungslehre, 2. Auflage, Berlin 1966, S. 39 .

38 Vgl. zu dieser Gleichsetzung von neoklassischer Theorie und Inzidenzanalyse auch P. Mieszkowski: Tax Incidence Theory: The Effects of Taxes on the Distribution of Income, in: Journal of Economic Literature, Bd. VII, 1969, S.1103.

39 Vgl. R.A.Musgrave: Finanztheorie, 2. Auflage, Tübingen 1969, S.163-165.

40 Nur der historischen Entwicklung entsprechend sei hier auf Steuern abgestellt, obwohl alle staatlichen Budgetoperationen Gegenstand von Inzidenzanalysen seln können.

41 Vgl. hlerzu R.A. Musgrave, a.a.O., S.177, FN 1) und H. -C.Recktenwald, a.a.O., S. 48 .

42 Vgl. R.A.Musgrave, a.a.O., S.162. So auch Break: "His (Musgraves d.V.) concept of incidence as the changes brought about by a given public finance instrument in the distribution of real income available for private use is 
an natural starting point for any discussion of the topic." G.F.Break: The Incidence and Economic Effects of Taxation, in: A.S.Blinder, R.S. Solow, G.F.Break, P.O.Steiner, D.Netzer: The Economics of Public Finance. Studies of Government Finance, Brookings Institution, Washington D.C. 1974, S. 123.

43 Vgl. R.A.Musgrave, a.a.O., S.155 und R.A.Musgrave/P.B. Musgrave: Public Finance in Theory and Practice, New York 1973, S.354-356.

44 Vgl. J.A.Pechman und B.A. Okner: Who Bears the Tax Burden? Studies of Government Finance, Brookings Institution, Washington D.C. 1974, S.27. S.a. G.F.Break: The Incidence and Economic Effects of Taxation, a.a.O., S.126.

45 G.E.McLure: Tax Incidence, Macroeconomic Policy and Absolute Prices, in: Quarterly Journal of Economics, Band 84, 1970, S.266.

46 Vgl. W. Kitschler: Entwicklungslinien der neueren Steuerinzidenzlehre, Diss. Mainz 1965, S. 136 und H. -C.Recktenwald, a.a. O., S. 67.

47 Vgl. R.A.Musgrave: Finanztheorie, a.a.O., S. 164/65.

48 Vgl. W.Kitschler, a.a.O., S.116.

49 Siehe hierzu auch P.Hövelmann: Die Verteilungseffekte von Gewinnsteuern in angebots - und nachfragetheoretischen makroökonomischen Modellen, Diss. Freiburg 1973, S. 25 und H.Fecher, a.a. O., S. 125/126.

50 "Price stability would require an assumption not of neutral monetary policy, ...., but of positive government action designed precisely to ensure such price stability." G. Parravicini: Outline of a Monetary Approach to the Theory of Tax Incidence, in: Banca Nazionale del Lavoro, Band 10, 1957, S. 268.

51 Es wird hier auf die für den deutschen Sprachraum grundlegende Terminologie von Recktenwald zurückgegriffen. Vgl. hierzu H. -C.Recktenwald, a.a.O., S.38-51. Zu den englischen Ausdrücken vgl. R.A. Musgrave u. P.B.Musgrave, a.a.O., S. 354/355.

52 So G.Blümle: Verteilungstheorie und makroökonomische Steuerüberwäl zungslehre, in: Jahrbuch für Sozialwissenschaft, Band 18, 1967, S.117. S.a. C.Koellreuter: Zur Theorie der internationalen Steuerinzidenz. Ein neoklassischer und ein postkeynesianischer Beitrag, Zürich 1973, S.9.

53 Durch Vergleich der formalen oder der gesetzlichen Inzidenz mit der effektiven läßt sich der jeweilige Grad der Überwälzung ermitteln. Vgl. H. -C. Recktenwald, a.a.O., S. 44 .

54 Hake charakterisiert diesen Ansatz als "aus der Sicht des Finanzamts". Vgl. W.Hake: Umverteilungseffekte des Budgets, Göttingen 1972, S.62.

55 Vgl. P.Hövelmann, a.a.O., S. 39. 
56 "Daß Musgrave die kreislauftheoretische Untersuchung meidet, führt ihn zu einigen Inzidenzkonstruktionen, die sich innerhalb der Kreislaufanalyse als verabsolutiert erweisen." H.Fecher, a.a.O., S.124, FN 66).

57 R.A.Musgrave, a.a.O., S. 159/160.

58 Im klassischen System wären dies die Veränderungen der relativen Preise, im kompensatorischen die des Preisniveaus. Dieser Einwand ist allerdings dann nicht gravierend, wenn diese Effekte größenmäßig vernachlässigt werden können.

59 Wobei hier die Wirkungen der Abschaffung der einen mit der Einführung der anderen Finanzierungsmaßnahmen verglichen werden, da von einem gegebenen Budget ausgegangen wird.

60 H.Fecher, a.a.O., S. 126.

61 Dies entspricht Musgrave's "Inzidenz des ausgeglichenen Budgets", wo die Verteilungseffekte der öffentlichen Leistungen unberücksichtigt bleiben.

62 So auch G.F.Break, a.a.O., S.128.

63 So greift die Inzidenzanalyse im klassischen System hilfsweise zur Annahme, daß die öffentliche Güterverwendung die gleiche sei wie bei alternativer privater Verwendung. Vgl. J.A.Pechman u. B.A.Okner, a.a.O., S.29.

64 Vgl. P.N.Rasmussen: On the Interrelations between Growth and the Distribution of Income, in: J.Marchal u. B.Ducros (Hrsg.): The Distribution of National Income, London/New York 1968, S.526/527.

65 So etwa H.Reinhardt: On the Incidence of Public Debt, in: Social Research, Band 12, 1945, S.205ff. J.G.Head: The Theory of Debt Incidence, in: Rivista di Diritto Finanzlario e Scienza delle Finanze, Band 26, 1967, S.175ff. W.Hake, a.a.O., S.72ff. Der Begriff der Inzidenz der Staats verschuldung als Synonym für die Verteilungswirkungen wird auch noch bei einer Reihe anderer Autoren verwendet.

66 Vgl. N.Andel: Zur These von den unsozialen Verteilungswirkungen öffentlicher Schulden, in: Public Finance, Band 24, 1969, S.69ff.

67 Der Hinweis erscheint wichtig, daß hier eine Differentialinzidenzbetrachtung aus verteilungspolitischer Sicht gefordert wird. Diese Begründung hebt sich von der der klassischen Inzidenztheorie ab, wo der Vorzug der Differentialinzidenz darin gesehen wird, daß der Ressourcentransfer in den staatlichen Bereich nicht mit in die Analyse eingeht.

68 Entgegen der Behauptung Andels (a.a.O., S.73) klingt ein solcher Vergleich mit alternativen Steuererhöhungen in der Schuldaufnahmephase bei einigen Autoren an: Vgl. J.M.Clark: The Cost of the World War to the American People, New Haven 1931, S.76. J.Heck: Die Problematik der Staatsschuldentilgung in der Theorie vom Merkantilismus bis zur Gegen- 
wart, Diss. Frankfurt 1954, S. 324. J.Wiseman: The Logic of National Debt Policy, in: J.M.Ferguson (Hrsg.): Public Debt and Future Generations, a.a.O., S.180, E.S.Phelps: Fiscal Neutrality toward Economic Growth, New York 1965, S.41/42.

69 Vgl. H.G.Koblitz: Einkommensverteilung und Inflation in kurzfristiger Analyse, Berlin-New York 1971, S.155.

70 Vgl. E.Scheele: Theorie der Einkommensverteilung, in: Kompendium der Volkswirtschaftslehre, W.Ehrlicher, I. Esenwein-Rothe, H.Jürgensen u. K. Rose (Hrsg.) 5. Auflage, Göttingen 1975, S. 330.

71 Vgl. E.Scheele: Einkommensverteilung und Wirtschaftswachstum, Tübingen 1965 , S. 43 .

72 So bei A. Oberhauser u.a. in: Staatsschuldentilgung als finanzpolitisches Instrument, in: Finanzarchiv, NF Band 24, 1965, S.283ff. Gewinnorientierte Einkommenspolitik und Staatsverschuldung, in: H.Haller (Hrsg.): Theorie und Praxis des finanzpolitischen Interventionismus, Fritz Neumark zum 70. Geburtstag, Tübingen 1970, S.529ff. O.Gandenberger: Die Wirkung des öffentlichen Kredits auf die Einkommensverteilung, in: Das Wirtschaftsstudium, 3. Jg., 1974, S. 383ff. W.Wittmann: Einführung in die Finanzwissenschaft III. Teil, 2. Auflage, Stuttgart 1976, S.25/26.

73 So bei G. Blümle: Theorie der Einkommensverteilung, a.a.O., S.153.

74 Diesem Umstand wird noch bei der Interpretation der Verteilungsergebnisse Bedeutung zukommen.

75 Die Staatsausgaben sind hier als Ausgabenart sui generis erfaßt, was of fen läßt, ob es sich um Investitions- oder Konsumgüter handelt. Auf die damit verbundenen Anforderungen an die Elastizität der Produktionsstruktur wurde schon hingewiesen. Die Symbole stehen für: $Y=$ Nettosozialprodukt, $S=$ gesamtwirtschaftliche Ersparnis, I=private Nettoinvestitionen, $\mathrm{Y}_{\mathrm{u}}=$ Einkommen der Unternehmer, $\mathrm{Y}_{\mathrm{nu}}=$ Einkommen der Arbeitnehmer.

76 Da auch später nur auf das Privatvermögen abgestellt wird, spielt die Verwendungsart staatlicher Ausgaben (investiv oder konsumtiv) keine Rolle. Siehe zu diesen Zusammenhängen A. Oberhauser: Finanzpolitik und private Vermögensbildung, Köln und Opladen 1963, S.26/27.

77 Es erscheint wesentlich, darauf zu verweisen, daß die in dieser Beziehung enthaltene Größe $\mathrm{K}_{\mathrm{St}}$ als gütermäßiger Anspruch an das Sozialprodukt $\mathrm{zu}$ interpretieren ist.

78 Vgl. G.Dieckheuer: Eine dynamische Analyse des "Crowding-out"-Effektes zusätzlicher Staatsausgaben, in: Finanzarchiv, NF Band 33, 1974/75, S. 387ff.

79 Womit eine, wie auch von der klassischen Schuldtheorie unterstellt, zinsunelastische Kapitalnachfrage des Staates impliziert ist. So etwa bei O.Gandenberger: Öffentlicher Kredit und Einkommensverteilung, a.a.O., S.11. 
80 Vgl. W. Mückl: Gleichgewichtswachstum, Einkommens - und Vermögens verteilung, Tübingen 1975, S.10.

81 Vgl. N.Kaldor: Alternative Theories of Distribution, in: N.Kaldor: Essays on Value and Distribution, London 1960, S.209 und 227.

82 Hier ist zusätzlich die staatliche Neuverschuldung zu berücksichtigen.

83 Dies stellt in der Entwicklung der Verteilungstheorie einen entscheidenden Schritt dar, da die klassische Theorie davon ausging, daß nur die Unternehmer Ersparnisse bilden. Vgl. H.G.Koblitz, a.a.O., S.160.

84 Vgl. N.Kaldor: Alternative Theories of Distribution, a.a.O., S.229, Fn 1). Gerade dies wurde zum Kritikpunkt, da die Annahme einer propensityGröße für unverteilte Gewinne nicht sinnvoll sei. So G.Bombach: Die verschiedenen Ansätze der Verteilungstheorie, in: E.Schneider (Hrsg.): Einkommensverteilung und technischer Fortschritt, Schriften des Vereins für Socialpolitik, NF Band 17, Berlin 1959, S.136/137.

85 Pasinetti wies darauf hin, daß die Annahme einer Ersparnisbildung der Arbeitnehmer dann auch erfordert, daß deren Vermögenseinkommen berücksichtigt werden. Pasinetti nimmt nun unabhängig von der Einkommensart eine für die jeweilige Gruppe repräsentative Sparneigung an, womit eine Erklärung der institutionellen Verteilung gegeben ist. Vgl. L. L. Pasinetti: Rate of Profit and Income Distribution in Relation to the Rate of Economic Growth, in: The Review of Economic Studies, Band 29, 1962, S.267ff. S.a. J.Kromphardt: Kapitalbildung in Arbeitnehmerhand und Einkommensverteilung im Gleichgewicht, in: Zeitschrift für die gesamte Staatswissenschaft, Band 122, 1966, S.247ff und die sich daran anschließende Diskussion zwischen Kromphardt, Mückl und Neumann.

86 Oben wurde schon auf die Interpretation dieses Ansatzes als Erklärung der institutionellen Verteilung hingewiesen. Kaldor selbst interpretiert seinen Ansatz nicht eindeutig, doch scheint er die Erklärung der funktionellen Verteilung zu favorisieren. Vgl. N.Kaldor: Marginal Productivity and the Macroeconomic Theories of Distribution. Comment on Samuelson and Modigliani, in: The Review of Economic Studies, Band 33, 1966, S. 310. Siehe dazu auch Mückl, der die Möglichkeit verschiedener Interpretationen aufzeigt. W. Mückl, a.a.O., S.23-29.

87 Dabei soll hier ausschließlich auf Nettogrößen abgestellt sein: $Y=$ Nettosozialprodukt, I $\mathrm{pr}=$ Nettoinvestitionen,

$\mathrm{Y}_{\mathrm{u}}, \mathrm{Y}_{\mathrm{nu}}=$ Einkommen der Unternehmer, Arbeitnehmer

$\mathrm{S}_{\mathrm{u}}, \mathrm{S}_{\mathrm{nu}}=$ Ersparnis der Unternehmer, Arbeitnehmer

$s_{\mathrm{u}}, \mathrm{s}_{\mathrm{nu}}=$ marginale (und durchschnittliche) Sparneigung der beiden Gruppen

s $_{\text {st }} \quad=$ Stillegungskoeffizient des Staates

88 Vgl. N.Kaldor: Alternative Theories, a.a.O., S. 230.

89 Vgl. N.Kaldor: Alternative Theories, a.a.O., S.232/233. 
90 So W.Albers: Die automatische Stabilisierungswirkung der Steuern - Möglichkeiten und Problematik in der Bundesrepublik Deutschland, in: Jahrbücher für Nationalökonomie und Statistik, Band 180, 1967, S.102 und K. -P. Fox: Die Berücksichtigung von Preisniveausteigerungen bei der Beurteilung konjunktureller Wirkungen öffentlicher Haushalte, in: Finanzarchiv, NF Band 33, 1974/75, S.437.

91 Der Parameter $\mathbf{s}_{\mathrm{St}}$ soll also als Stillegungsindex interpretiert werden, nicht aber als Sparneigung im Sinne einer Propensity-Größe. Vgl. den Hinweis bei Kath, daß ein rationales Sparmotiv des Staates aus der Einsicht in Kreislaufzusammenhänge erklärbar sei. D. Kath: Die Bedeutung des staatlichen Sparens für die Kapitalbildung und das Wirtschaftswachstum, Berlin 1968, S.24. Siehe dazu auch G. Blümle: Verteilungstheorie und makroökonomische Steuerüberwälzungslehre, a.a.O., S. 203/204.

92 Vgl. N.Kaldor: Alternative Theories, a.a.O., S.230.

93 Eine solche Annahme findet sich bei Hövelmann, a.a.O., S. 55.

94 Vgl. A.K.Sen: Neo-Classical and Neo-Keynesian Theories of Distribution, in: Economic Record, Band 39, 1963, S.63.

95 So geht Koblitz davon aus, daß sich im Laufe der Verteilungsänderungen die Sparneigungen verändern müssen. So H.G.Koblitz, a.a.O., S.169/170.

96 In den unterschiedlichen Wirkungsabläufen, die sich alle hinter einer identischen Primärverteilung verbergen, werden die Grenzen der Aussagefähigkeit dieses Verteilungsmaßes deutlich.

97 Dieser Sachverhalt stellt ein generelles Problem staatlicher Sparförderung dar - und in diesen Rahmen stellten wir obige Verknüpfung. Vgl. M.Bitz: Pläne und Maßnahmen zur Vermögensbildung, Köln 1971, S.140ff und G.H. Milbradt: Ziele und Strategien des debt management, Baden-Baden 1975, S.99. Eine ausführliche Diskussion über die Reaktionen des Sparverhaltens bei vermögenspolitischen Maßnahmen findet sich bei U.Schillert: Gewinne als Quelle der Vermögenspolitik? Berlin 1976, S.68-132.

98 Wobei das Auftreten von Preiseffekten und die damit verbundenen negativen Verteilungswirkungen davon abhängen, ob diese Mehrersparnis im Kreislaufgeschehen zwangsweise gebildet werden mußte.

99 Im gerade aufgezeigten Fall der freiwilligen Mehrersparnis ergibt sich im kreislauftheoretischen Modell gleichfalls keine Änderung der Einkommensverteilung. Dennoch sind diese Fälle streng zu unterscheiden, da im klassischen System die Anpassung in Form eines Automatismus erfolgt, wohingegen die oben beschriebene Verhaltensänderung der privaten Wirtschaftssubjekte als unwahrscheinlich einzuschätzen ist.

100 Da von einer gegebenen (realen) staatlichen Kreditnachfrage ausgegangen wird, können nur die privaten Investitionen tangiert werden. 
101 Vgl. W.Krelle: Die Investitionsfunktion, in: Jahrbücher für Nationalökonomie und Statistik, Band 172, 1960, S. 346/347.

102 Siehe hierzu E. Preiser: Investition und Zins, in: E. Preiser: Bildung und Verteilung des Volkseinkommens, 3. Auflage, Göttingen 1963, S.176-196.

103 Vgl. E.Preiser, a.a.O., S. 185 u. 203 und W.Krelle, a.a.O., S. 349.

104 "So alt die Theorie von der Zinsabhängigkeit der Investitionen ist, so schlecht bewährte sie sich in der Praxis. Nur bei sehr wenigen Wirtschaftszweigen hat sich der Zinsfuß als signifikant für die Investition herausgestellt". W.Krelle: Verteilungstheorie, Tübingen 1962, S.145. Ähnlich D.Duwendag: Investitionsdeterminanten im Wohnungs - und Städtebau während der deutschen Nachkriegsperiode, in: Jahrbuch für Sozialwissenschaft, Band 18, 1967, S.230. Diekwisch zeigt bei einer vergleichenden Darstellung verschiedener Investitionshypothesen für die Bundesrepublik auf, daß gerade dem Faktor Zins kaum ein Einfluß zuzuschreiben ist. S.Diekwisch: Das Zeitmoment in der antizyklischen Finanzpolitik der Bundesrepublik, Diss. Freiburg 1974, S.106 und 108.

105 Wie noch zu zeigen ist, sind diese Faktoren auch für die intertemporalen Verteilungseffekte verantwortlich.

106 Vgl. A.S.Eichner und J.A.Krengel: An Essay on Post-Keynesian Theory: A New Paradigma in Economics, in: The Journal of Economic Literature, 1975, Band 13, S. 1300.

107 Der hier verwendete Endogenitätsbegriff stellt ausschließlich auf das Bankenverhalten ab, nicht aber auf durch das Publikum bewirkte Veränderungen. Vgl. zu letzterem Aspekt J.Siebke: Fiskalistische oder Monetaristische Konjunkturstabilisierung? in: Gahlen und Schneider (Hrsg.): Grundfragen der Stabilitätspolitik, Tübingen 1974, S.236/237.

108 Indem diese nämlich die Voraussetzungen der klassischen Schuldtheorie zu schaffen hat.

109 Diese Verschuldungsform spielt auch aus institutionellen Grüden in der Bundesrepublik keine Rolle, da hier, nicht zuletzt aufgrund historischer Erfahrungen, in Form der Kassenkredite nur vergleichsweise enge Spielräume gegeben sind. Vgl. hierzu B.Gaude: Die Mechanismen der Zentralbankgeldschöpfung und ihre Kontrollierbarkeit durch die Zentralbank, Berlin 1969, S. 78/79.

110 In der Literatur wird häufig auf diese Verschuldungsform zurückgegriffen, da damit eine Kaufkraftabschöpfung erreicht werde, So etwa H. -D. Hessler: Gegenwartsprobleme staatlicher Anleihepolitik, Köln und Opladen 1964, S.65. J.M.Buchanan und M.R.Flowers: Public Finances, a.a.O., S. 334 .

111 Diese Verschuldungsform spielt in der Bundesrepublik eine quantitativ untergeordnete Rolle. Vgl. W.Dreißig: Zur Entwicklung der öffentlichen Finanzwirtschaft seit dem Jahre 1950, a.a. O., S.730-733. 
112 Da wir von einem Gleichgewicht in einer Vollbeschäftigungssituation ausgehen, spielt die Möglichkeit einer Kreditgewährung aufgrund zusätzlicher Ersparnisbildung keine Rolle, sieht man vom unter III.2.f. geschilderten Fall ab.

113 Wobei diese Annahme von der Möglichkeit abhängt, verzinsliche Sekundärliquidität $\mathrm{zu}$ halten. Vgl. Jahresgutachten des Sachverständigenrates 1974, TZ 386.

$114 \mathrm{Vgl}$. H. -J.Jarchow: Theorie und Politik des Geldes. II. Geldmarkt und geldpolitische Instrumente, Göttingen 1974, S.13, FN 3) und D.Duwendag, K. -H.Ketterer, W.Kösters, R. Pohl u. D.B.Simmert: Geldtheorie und Geldpolitik, Köln 1974, S.38ff.

115 Allerdings muß auch bei festen Wechselkursen der Interventionspunkt erreicht sein, da andernfalls kein zusätzliches Zentralbankgeld entsteht.

116 "In der Bundesrepublik sind die weitaus wichtigsten Quellen der Bankenliquidität - neben der alle übrigen sich fast immer als unbedeutend ausnahmen - die Überschüsse im internationalen Zahlungsverkehr...". W. Neubauer: Strategien, Techniken und Wirkungen der Geld- und Kreditpolitik, Göttingen 1972, S. 31 .

117 Diese idealisierende Sicht abstrahiert einerseits vom Blockfloating und andererseits vom "managed floating". Vgl. zu den sich realiter ergebenden Schwierigkeiten Geschäftsbericht der Deutschen Bundesbank für das Jahr 1975, S.54/55. S.a. G.Fels: Fiskalistische oder monetaristische Konjunkturstabilisierung? in: B.Gahlen und H.K.Schneider (Hrsg.): Grundfragen der Stabilitätspolitik, Tübingen 1974, S.209.

118 Vgl. zur Bedeutung des Übergangs zu flexiblen Wechselkursen für die Geldpolitik der Bundesrepublik N.Kloten: Erfolg und Mißerfolg der Stabilisierungspolitik (1969-1974), in: Deutsche Bundesbank (Hrsg.): Währung und Wirtschaft in Deutschland 1876-1975, Frankfurt 1976, S. 653 u. 658 .

119 Müßten die Banken die Kreditbeträge auf Zentralbankkonten überweisen, so hätte dies zunächst einen höheren Zentralbankgeldbedarf zur Folge. Allerdings würde dieses Zentralbankgeld mit der Verausgabung wieder in das Bankensystem zurückfließen, so daß die Wirkungen für das gesamte Bankensystem dem hier aufgezeigten entsprechen, wenn sich auch für einzelne Banken relevante Unterschiede ergeben mögen. Vgl. W.A.S. Koch: Die Gläubigerstruktur als Ansatzpunkt für ein staatliches debt management, Meisenheim am Glan 1972, S.170ff. B.Rahmann: Grundlagen konjunkturbeeinflussender Haushaltspolitik. Ein Beitrag zu einer Theorie der Budgetwirkungen, Berlin 1972, S. 54. 
120 Für die private Investitionsnachfrage kommt diesem Zinssatzeffekt insofern Bedeutung $z u$, als u.U. Investitionen, für die der Zins einen bedeutenden Kostenfaktor darstellt, nicht mehr durchgeführt werden, während bei den anderen Investitionsnachfragern aufgrund der erhöhten Investitionsneigung keine Verdrängungseffekte zu erwarten sind.

121 Dabei gilt es allerdings zu beachten, daß die Banken häufig ein bestimmtes Maß an Sekundärliquidität nicht unterschreiten. Ein solches Verhalten war etwa bis zum Jahr $1970 \mathrm{zu}$ beobachten, in der Folgezeit gingen diese Reserven allerdings auch auf Null zurück. Vgl. zu den Schwierigkeiten der Ermittlung einer "normalen" Liquiditätsquote C.Köhler: Probleme der Zentralbankgeldmengensteuerung, Arbeitspapiere des Instituts für Empirische Wirtschaftsforschung, Heft 4, Berlin 1976, S. 33-36.

122 M.Willms: Der Einsatz der Staatsschuld als geldpolitisches Instrument, in: Kredit und Kapital, 1. Jg., 1968, S.416/17. S.a. W. Neubauer, a.a. O., S. 183 .

123 Vgl. G. Lindvers: Defizitfinanzierung staatlicher Ausgaben und Geldpolitik im Konjunkturablauf. Analyse des Wirkungszusammenhangs auf der Grundlage alternativer monetärer Hypothesen, Diss. Freiburg 1971, S. 56.

124 So läßt sich eben aus dem Umstand, daß private Wirtschaftssubjekte staatliche Papiere kaufen, noch nicht schließen, daß dadurch den privaten Investitionen "Sparmittel" entzogen würden. So etwa H. -D.Hessler, a.a. O., S. 65 .

125 Vgl. Jahresgutachten des Sachverständigenrates 1974, TZ 423 und 424.

126 Jahresgutachten des Sachverständigenrates 1974, TZ 378.

127 So erreichte $1973 / 74$ die freie Liquiditätsquote der Banken einen Wert nahe Null. Vgl. N.Kloten, a.a.O., S.658. A. Oberhauser: Die Liquiditätstheorie des Geldes als Gegenkonzept zum Monetarismus, in: Kredit und Kapital, 10. Jg., 1977, S.207ff.

128 Vgl. W. Neubauer, a.a.O., S. 134.

129 Diesbezügliche Zweifel klingen bei Willms an, während sich Schlesinger recht optimistisch äußert. Vgl. M.Willms: a.a.O., S.410 und H.Schlesinger: Fiskalistische oder monetaristische Konjunkturstabilisierung? in: B. Gahlen und H.Schneider (Hrsg.): Grundfragen der Stabilitätspolitik, Tübingen 1974, S. 223/224.

130 H.Weise: Probleme der Geld- und Kreditpolitik im Lichte des RadcliffeReports, Tibingen 1964, S. 154.

131 Auf diese Gefahr verweist N.Kloten, a.a.O., S.660.

132 Dies gilt auch für den Fall der freiwilligen zusätzlichen Vermögensbildung, der aber als nicht sonderlich wahrscheinlich vernachlässigt wird. 
133 Es sei hier daran erinnert, daß mit Hilfe des Kaldor-Modells lediglich die Verteilungsstruktur bei gegebenem Verteilungsniveau bestimmt werden kann.

$134 \mathrm{Da}$ in einer solchen Situation mit keinem Crowding-out zu rechnen ist, sollte als konjunkturpolitisch bedingte Selbstverständlichkeit unterstellt werden dürfen.

135 Durch deren Verausgabung entsteht im Rahmen des einsetzenden Multiplikatorprozesses wiederum Nachfrage, die bezüglich der Verteilungswir kungen analoge Fragen aufwirft.

136 Theoretisch könnte im völlig unrealistischen Extremfall die notwendige Ersparnisbildung auch ausschließlich aus den zusätzlichen Lohneinkommen geleistet werden - wenn nämlich alle zusätzlichen Kreislaufeinkom men der Unternehmer wieder zu Lohnauszahlungen verwendet würden.

137 Vgl. Bombach: Die verschiedenen Ansätze ..., a.a.O., S.100.

138 Es wurde bislang unter der Annahme unverändert gebliebener Verhaltensparameter argumentiert. $\mathrm{Da}$ auch in einer Aufschwungsphase eine $\mathrm{Zu}-$ nahme der Sparleistungen der Arbeitnehmer diesen Verteilungsvorteile bringt, hat mit der speziellen Situation der Unterbeschäftigung nichts $z u$ tun.

139 Diese finden in der Spezifikation der gesamtwirtschaftlichen Produktionsfunktion ihren Niederschlag.

140 Eine Annahme, die bei erstem Augenschein schon realitätsfremd zu sein scheint - vgl. zu dieser Kritik: O.Morgenstern: Thirteen Critical Points in Contemporary Economic Theory: An Interpretation, in: Journal of Economic Literature, Band 10, 1972, S. 1185.

141 Vgl. G. Blümle: Theorie der Einkommensverteilung, a.a.O., S.131.

$142 \mathrm{Zu}$ den langfristigen Wirkungen innerhalb eines neoklassischen Modells vgl. G. Tolkemitt: a.a.O.

143 Daß sich die Zinszahlungen einkommensmäßig erst zu Beginn der nächsten Periode auswirken, spielt für die innere Logik dieses Ansatzes ohnehin keine Rolle.

144 O. Gandenberger: Öffentlicher Kredit und Einkommensverteilung, in: Finanzarchiv, NF Band 29, 1970, S.1-16.

145 Vgl. K. -H.Hansmeyer: Der öffentliche Kredit, 2. Auflage, Frankfurt 1970, S.108. G.Schmölders: Finanzpolitik, 3. Auflage, Berlin-HeidelbergNew York 1970, S.409/410. H.Zimmermann und K. -D. Henke: Finanzwissenschaft. Eine Einführung in die Lehre von der öffentlichen Finanzwirtschaft, München 1975, S.201. R. Peffekoven: Einführung in die Grundbegriffe der Finanzwissenschaft, Darmstadt 1976, S.77. M.E.Kamp, W. Schönebeck, D.Smolinski, H.Weiler: Öffentliche Finanzwirtschaft, Köln 
1975, S.216. W.Wittmann: Einführung in die Finanzwissenschaft, III. Teil, Stuttgart 1976, 2. Auflage, S.23-27.

146 Stellvertretend sei folgendes Zitat von Peffekoven wiedergegeben: "Solche unerwünschten Einkommenswirkungen (des Transferansatzes d.V.) könnten jedoch vermieden oder doch wenigstens verringert werden, wenn durch geeignete Maßnahmen auch einkommensschwächere Schichten am Erwerb von Anleihetiteln und folglich am Zinsertrag beteiligt würden. Neben diesen Wirkungen auf die personelle Einkommensverteilung gehen von einer Kreditfinanzierung auch Einfluisse auf die funktionelle Einkommensverteilung aus." R.Peffekoven, a.a.O., S.77. Hieraus wird deutlich, daß die mit Gandenbergers Ansatz verbundene verteilungstheoretische Fundierung nicht richtig erkannt wurde.

147 Dies ist auch aus seinem primären Untersuchungsziel erklärbar, die Unhaltbarkeit des Transfer-Ansatzes nachzuweisen.

148 Zudem muß unterstellt werden, daß keine Horte aktiviert werden.

149 Hier stellt sich die Frage, wie ein solches Verhalten in ein Modell zu integrieren ist, das sonst durch strenge Rationalitätsüberlegungen gekennzeichnet ist. Wollte man ein privatem Investitionsverhalten analoges Kalkül auf die staatliche Kreditnachfrage anwenden, müßten bestimmte Vorstellungen iber eine soziale Rendite vorliegen. Dem entspricht aber die übliche Unterstellung der Zinsunempfindlichkeit nicht, die die staatliche Nachfrage als exogenen Faktor einführt.

150 Da wir auch hier dem Konzept der Budgetinzidenz folgen, ergibt sich ein Unterschied zur Darstellung Gandenbergers, bei der sich aufgrund einer Differentialinzidenzbetrachtung eine Verschiebung der Angebotsfunktion ergibt.

151 Es wird eine möglichst allgemeine Konstellation angenommen, da es um die grundsätzlichen Zusammenhänge geht. So ist darauf hinzuweisen, daß Gandenberger sowohl für die Kapitalnachfrage- als auch Angebotsfunktion eine nur geringe bzw. nicht vorhandene Zinselastizität als realistisch unterstellt. Vgl. O.Gandenberger, a.a.O., S.11/12 und ders.: Die Wirkung des Öffentlichen Kredits auf die Einkommensverteilung, in: Das Wirtschaftsstudium, 3.Jg., 1974, S.384.

152 Dieser Personenkreis wird dabei nicht mit dem der Zeichner der staatlichen Papiere identisch sein.

153 "... es könnte auch sekundäre Auswirkungen auf die Höhe der Zinseinkommen, die Lohn-Zins-Relation und damit auf die Einkommensverteilung künftiger Perioden haben; solche Einflüsse können jedoch als unbedeutend ausgeschieden werden, wenn wir es mit gesamtwirtschaftlichen Produktionsfunktionen $\mathrm{zu}$ tun haben, deren Substitutionselastizität annähernd gleich eins ist". O.Gandenberger, Öffentlicher Kredit und Einkommensverteilung, a.a.O., S.14. 
154 In der Gleichung III.15. des Kaldor-Ansatzes entfiele dann das additive Glied. Mit den hier getroffenen Aussagen wird der Wirkungsmechanismus Kaldors erstmals in kontraktiver Richtung unterstellt. Als erste Begründung kann man anführen, daß durch die Vorgänge der Vorperiode auch tatsächlich Kreislaufgewinne vorhanden sind, die für Verteilungs änderungen in umgekehrter Richtung zur Verfügung stehen.

155 Oben wurde darauf hingewiesen, daß der Kaldor-Ansatz lediglich die Struktur der Verteilung bestimmt, da durch die Definition als Realmodell die Niveaus nominaler Größen nicht bestimmbar sind. Legt man diesen Ansatz nun der Betrachtung einer wachsenden Wirtschaft zugrunde, so ist $z u$ beachten, daß auch dann alle Größen in Form realer Quoten erfaßt sind.

156 Wobei nochmals daran zu erinnern ist, daß die Verteilungswirkungen der Zinszahlungen noch völlig unberücksichtigt sind.

157 Vgl. W.Vickrey: The Burden of the Public Debt: Comment in: J.M. Ferguson: Public Debt and Future Generation, a.a.O., S.78. Vgl. auch die Untersuchung der Verteilungswirkungen einer einmaligen staatlichen Schuldaufnahme für eine stationäre Wirtschaft bei G.Tolkemitt, a.a.O., S. 34-41.

158 Vgl. zu der im Zeitablauf unterschiedlichen Behandlung staatlicher Zinszahlungen R.Ruggles: Volkseinkommen und volkswirtschaftliche Gesamtrechnung, Wien/Stuttgart 1952, S.83. U.Schumacher: Nationalbudget und öffentlicher Haushalt, Stuttgart 1958, S.47, FN 89. H.Haller: Finanzpolitik, 5. verb. Auflage, Tübingen 1972, S.35. D. Brümmerhoff: Gesamtwirtschaftliches Rechnungswesen, Köln 1975, S. 57/58. G.Bombach: Die öffentliche Finanzwirtschaft im Wirtschaftskreislauf, in: F. Neumark (Hrsg.): Handbuch der Finanzwissenschaft, Band 1, Lieferung 1, 3. gänzlich neubearbeitete Auflage, Tübingen 1975, S.68.

159 N. Andel: Zur These von den unsozialen Verteilungswirkungen öffentlicher Schulden, a.a.O., S.74. Neben der schon oben angesprochenen Forderung nach Berücksichtigung der Differentialinzidenz kommt Andels Aufsatz auch der Verdienst zu, auf die Unzulänglichkeit dieses Transfer-Ansatzes verwiesen $\mathrm{zu}$ haben.

160 So etwa J.Cohen: Distributional Effects of the Federal Debt, in: Journal of Finance, Vol. VI, 1951, S.267ff. W.I.Gillespie: Effect of Public Expenditures on the Distribution, in: R.A.Musgrave (Hrsg.): Essays in Fiscal Federalism, Washington 1965, S.157ff. D.C.Miller: Taxes, The Public Debt and Transfers of Income Urbana 1950 W.Hake, a.a.O., S. 201-203.

161 So O.Gandenberger: Öffentlicher Kredit und Einkommensverteilung, a.a.O., S. 2 und G. Tolkemitt, a.a.O., S. 34 . 
162 Wissenschaftlicher Beirat beim Bundeswirtschaftsministerium: Kriterien und Konsequenzen der Staatsverschuldung, 1968, wieder abgedruckt in: H.C.Recktenwald (Hrsg.): Finanzpolitik, Köln-Berlin 1969, S.427 und 431 .

163 So etwa H.Haller: Finanzpolitik, 5. Auflage, Tübingen 1972, S.198/199. H.Kock: Staatsverschuldung, Wachstumszyklen und Einkommensverteilung, in: B.Gahlen (Hrsg.): Wachstumszyklen und Einkommensverteilung, Tübingen 1974, S.282/283. C.A.Andreae und K.Schlögl: Finanzpolitik, Stuttgart 1975, S.130. B. Molitor: Unsoziale Staatsverschuldung? in: B. Molitor: Verteilungspolitik in Perspektive, Hamburg 1975, S.113-115. W. Ehrlicher: Finanzwissenschaft, in: W. Ehrlicher u.a. (Hrsg.): Kompendium der Volkswirtschaftslehre Band 2, 4. Auflage, Göttingen 1975, S. 352 .

164 Von den möglichen Zinseffekten sei abstrahiert, da die private Ersparnisbildung als nicht zinsreagibel angenommen wird, zudem werden diese nicht der Staatsverschuldung zugeschrieben.

165 Auch wenn die Alternative einer Steuerfinanzierung bestünde, blieben diese und ihre möglichen (differentiellen) Wirkungen dem einzelnen verborgen. Vor dieser Fragestellung der Differentialinzidenz steht also nur die Legislative bzw. Exekutive. Denkmodelle klassisch liberaler Provenienz, die mit zunehmendem Informationsgrad des Einzelnen zu einer indifferenten Beurteilung von Steuer und Anleihe kommen, müssen als unrealistisch zurückgewiesen werden. Auch darin ist eine Begründung für das hier gewählte Inzidenzkonzept zu sehen.

166 Als "unerreichtes Vorbild", "... sich, wie insbesondere in Kriegszeiten, eine Welle der nationalen Begeisterung für die Zwecke der Finanzpolitik zunutze zu machen" bezeichnet Schmölders den in USA durchgeführten "War Bond Drive" während des Zweiten Weltkrieges. Doch soll hier von solchen Ausnahmesituationen abgesehen werden. Vgl. G.Schmölders: Finanz - und Steuerpsychologie, Hamburg 1970, S.210.

167 Vgl. W.Wittmann: Einführung in die Finanzwissenschaft III. Teil, Stuttgart 1972, S.26. Ein gleichfalls in diese Richtung zielender Einwand findet sich bei K.-D. Henke: Probleme der Staatsverschuldung, in: Zeitschrift für Wirtschafts - und Sozialwissenschaften 1973, 93.Jg., S. $62 / 63$.

168 Allerdings können sich unterschiedliche Zinshöhen ergeben, was aber für die kreislauftheoretische Analyse, die von einem gegebenen Zinsniveau ausgeht, nicht interessiert, da diese Effekte in der gesamtwirtschaftlichen Gewinnquote der Primärverteilung bereits enthalten sind. 
169 Dieses Vorgehen ist natürlich eng mit dem hier verwendeten methodischen Ansatz verbunden. So wäre dieser Kreislauf für eine Inzidenzanalyse der Zinssteuern in neoklassischer Sicht ohne Belang.

170 Erste Hinweise dieser Art finden sich nach Dorn schon bei Malthus und Lauderdale. Vgl. D.Dorn: Die Theorie der Staatswirtschaft in der englischen Klassik, Diss. Nürnberg-Erlangen 1968, S.205. Ferner bei S.E. Harris: The National Debt and the New Economics, New York/London 1947, S.9ff. J.Koulis: Wirtschaftliche Grundfragen des Staatskredits, Frankfurt 1948, S.90-92. D.C.Miller: Taxes, The Public Debt and Transfer of Income, Urbana 1950, S.3/4 u. 138. J.Heck: Die Problematik der Staatsschuldentilgung in der Theorie vom Merkantilismus bis zur Gegenwart, Diss. Frankfurt 1954, S.318/319. G.Häussler: Die Problematik der Last der öffentlichen Schuld, Diss. Graz 1961, S.119. H. -D.Hessler: Gegenwartsprobleme staatlicher Anleihepolitik, Forschungsberichte des Landes Nordrhein-Westfalen Nr. 1373, Köln-Opladen 1964 , S.72/73. N. Andel: Probleme der Staatsschuldtilgung, Berlin 1964, S.53/ 54. J.Hanschel: Kreislauftheorie und Staatshaushalt, Zürich 1967, S. 73 (und S.42). W.Wittmann: Einfuihrung in die Finanzwissenschaft, I. Teil, Stuttgart, S.93. W.H.Anderson: Financing Modern Government, Boston 1973, S.243. Ein unmittelbar auf Verteilungszusammenhänge und damit den Transferansatz in Frage stellender Hinweis findet sich bei A. Oberhauser: Der Investivlohn als Mittel der Verteilungspolitik, in: H. Arndt (Hrsg.): Lohnpolitik und Einkommensverteilung, Schriften des Vereins für Socialpolitik, NF Band 51, Berlin 1969, S.282/283.

171 Da von der Tilgung abstrahiert wird, impliziert dies eine unendlich große Zahl von Verzinsungsperioden.

172 Dabei müssen die ursprünglichen Einkommen der beiden Gruppen nicht homogen gewesen sein.

173 In gleicher Weise in ein kreislauftheoretisches Modell integrierte Steuersätze finden sich etwa bei G.Bombach: Das Problem der optimalen internationalen Arbeitsteilung bei unterschiedlicher Struktur der Steuersysteme mit einem Anhang von Hajo Riese, Düsseldorf 1962, S.32-38. W. Mückl, P. Baumgarten und K. -D.Schmidt: Die Beziehungen zwischen Wirtschafts struktur und Einkommensverteilung, Tübingen 1968. Vgl. ferner die bei P.Hövelmann a.a.O., S. 43 angegebenen Autoren.

174 Als Vergleichsgröße ist hier die Gleichung III.15. anzuführen, in der der zweite Summand entfällt, da nun $K_{S t}$ gleich Null zu setzen ist:

$$
\frac{\mathrm{Y}_{\mathrm{u}}}{\mathrm{Y}}=\frac{\mathrm{I}_{\mathrm{pr}} / \mathrm{Y}-\mathrm{s}_{\mathrm{nu}}}{\mathrm{s}_{\mathrm{u}}-\mathrm{s}_{\mathrm{nu}}}
$$

175 Auf die Frage, ob die weitergehende Stabilitätsbedingung in Analogie zu III.16. erfüllt ist, wird unten eingegangen. 
176 Womit impliziert wird, daß die private Investitionsgüternachfrage trotz dieser Nachfragereduktion real konstant bleibt.

177 Diese müssen natürlich nicht absolut, sondern können auch in Form ausbleibender bzw. schwächerer Preissteigerungen auftreten.

178 Entscheidend für dieses Verteilungsergebnis ist allerdings die strikte Verhaltensausrichtung an realen Größen. Gingen nämlich die nominale Konsumguiter - oder auch Investitionsgüternachfrage der Unternehmer nicht den Preisniveausenkungen entsprechend zurück, so ergäben sich erhöhte reale Ansprüche an das Sozialprodukt, die auch durchgesetzt werden könnten.

179 Auf den zweiten Bestandteil der Relation III.16. wird hier verzichtet, da sich hierfür keine Veränderung ergeben hat, d.h. die Bedingung $s_{u}>I / Y$ nach wie vor erfüllt sein muß.

180 Die Darstellung der Stabilitätsbedingungen geht auf Anderson zurück. Vgl. hierzu R.W.Anderson: A Note on Tax Incidence in a Macroeconomic Distribution Model, in: Rivista Internationale de Science Economiche e Commerciale, Band 16, 1969, S.1164ff.

181 Diese Ortslinie ergibt sich aus der Ungleichung $s_{u}-t_{u}\left(s_{u}-s_{n u}\right)>$ $\mathrm{I} / \mathrm{Y}>\mathrm{s}_{\mathrm{nu}}$, d.h. auch hier wird der zweite Teil der Bedingung als erfüllt vorausgesetzt.

182 Vgl, hierzu J.Kromphardt, a.a.O., S. 257.

183 Es wäre auch an die Möglichkeit zu denken, daß sich durch den Mechanismus der Preisniveauveränderungen Auswirkungen auf die Verhaltensparameter ergeben. Hier findet sich eine Beschränkung der Aussagen des Modells, daß nämlich der sekundäre Kreislauf nicht beliebige Aus maße annehmen kann, ohne daß die institutionelle Verteilung verändert würde.

184 Wobei zu berücksichtigen ist, daß eine Gewinnsteuer auch die Investitionsmöglichkeiten berühren kann.

185 Vgl. P.Hövelmann, a.a.O., S.93/94.

186 Vgl, hierzu S.Diekwisch, a.a.O., S.113/114.

187 Hierin war ein Unterschied zum grenzproduktivitätstheoretischen Ansatz zu sehen, wo Veränderungen der funktionellen und personellen Verteilung im Zeitablauf, wenn auch mit geringer werdendem Einfluß, nachwirken.

188 Denn selbst bei einem erfolgreichen, durch geldpolitische Eingriffe erzwungenen Crowding-out ist in der Nachfolgeperiode $z u$ erwarten, daß die private Investitionsnachfrage wieder die alte Höhe erreicht.

189 Mit dem Zurückdrängen privater Investitionen ist selbst dann ein relativer Wachstumseffekt verbunden, wenn die staatliche Ressourcenverwendung ebenso produktiv ist, da andernfalls (d.h. ohne Crowding-out) die Wachstumseffekte noch stärker gewesen wären. 
190 In diesem Sinne W. Wittmann: Einführung in die Finanzwissenschaft, III. Teil, Stuttgart 1972, S. 27.

191 G.Hedtkamp: Lehrbuch der Finanzwissenschaft, Neuwied und Berlin 1968, S.314. Nach Molitor handelt es sich dabei um eine "ideologisch besetzte Vorstellung". B. Molitor: Unsoziale Staatsverschuldung, in: B. Molitor: Verteilungspolitik in Perspektive, Hamburg 1975, S.121.

192 Vgl. dazu die Übersicht bei C.S.Shoup: Staatsverschuldung und künftige Generationen, in: H.C.Recktenwald (Hrsg.): Finanztheorie, Köln 1969, S. 470 .

193 Der angesprochene Einkommensverzicht würde sich in den andernfalls notwendig werdenden Steuerzahlungen manifestieren, d.h. die Ausgabeentscheidung des Staates wird als gegeben vorausgesetzt.

194 Auf die Bedeutung der Staatsauffassung für die unterschiedlichen Lastinhalte der mikro- und makroökonomischen Ansätze verweist K. Meister, a.a.O., S.157 FN 1) und S.161 FN 6).

195 Auch hier wird wieder die Bedeutung der Verausgabung deutlich.

196 Der Fall der außenwirtschaftlichen Verflechtung sei nach wie vor ausgeschlossen.

197 Sieht man von Situationen der Unterbeschäftigung und realem Kapitalimport $a b$.

198 Die Terminologie sowohl bezüglich des Wachstumsansatzes (bzw. Aggregate Investment Approach) als auch der Neuen Orthodoxie hat sich im Anschluß an Gandenbergers Aufsatz auch im deutschen Sprachraum durchgesetzt. Vgl. O.Gandenberger: Intertemporale Verteilungs wirkungen der Staatsverschuldung, a.a.O., S.189ff.

199 Man muß diese Entwicklung auch in Zusammenhang mit der durch Buchanan zu dieser Zeit aufgestellten Hypothese sehen, daß eine Lastverschiebung durch staatliche Verschuldung möglich sei. Mit der Weiterentwicklung des Standpunkts der Neuen Orthodoxie konnten nun vom Ergebnis her ähnliche Wirkungen aufgezeigt werden.

200 Überlegungen dieser Art finden sich bei folgenden Autoren: R.A. Musgrave: The Theory of Public Finance, New York 1959, S.577f. F. Modigliani: Long-run Implications of Alternative Fiscal Policies and the Burden of the National Debt, in: Economic Journal, Band 72, 1961, S.730ff, wiederabgedruckt in: J.M.Ferguson: Public Debt and Future Generations, a.a.O., S.107ff. H.Neisser: Is the Public Debt a Burden on Future Generations? in: Social Research, Band 28, 1961, S.730ff, wiederabgedruckt in: J.M.Ferguson, a.a.O., S.145ff. W.Vickrey: The Burden of the Public Debt: Comment, in: The American Economic Review, Band 51, 1961, S.132ff, wiederabgedruckt in: J.M.Ferguson, a.a. O., S. 74 ff. 
201 So auch O.Gandenberger: Intertemporale Verteilungswirkungen, a.a.O., S.192.

202 Könnte der Zinssatz, zu dem der Staat seine Anleihen aufnimmt, als Näherungsgröße für die gesamtwirtschaftliche Kapitalproduktivität angesehen werden und würden die privaten Investitionen in vollem Umfange der zusätzlichen Staatsausgaben zurückgedrängt werden, so wäre die Summe der Zinszahlungen des Staates ein brauchbarer Indikator für die Bruttoeinkommensverluste der kommenden Perioden.

Vgl. F.Modigliani, a.a.O., S.118. Siehe dazu kritische Einwände bei G. Tolkemitt, a.a.O., S.25.

203 In diesem Sinne auch E.J.Mishan, a.a.O., S.191 und 200 sowie J.M. Ferguson: Introduction, in: J.M.Ferguson (Hrsg.): Public Debt and Future Generations, a.a.O., S.8.

204 Denkt man andererseits an den Abbau beschränkt verfügbarer natürlicher Ressourcen, so wäre durch zu hohe Investitionen auch eine Belastung zukünftiger Generationen denkbar. So etwa R.A.Musgrave/P.B. Musgrave/L.Kullmer: Die öffentlichen Finanzen in Theorie und Praxis Band I, Tübingen 1975, S.123. H.-J.Hof und U.Wagner: Verteilungswirkungen externer Effekte - dargestellt am Beispiel verfahrensbedingter Umweltbelastungen, in: D.Cassel und H.J.Thieme: Einkommensverteilung im Systemvergleich, Stuttgart 1976, S.138.

Tolkemitt verweist in diesem Zusammenhang auf das Beispiel eines golden-age-Modells, in dem aufgrund eines zu hohen Kapitalstocks die Drosselung der Investitionen sogar das Erreichen eines höheren Konsumniveaus ermöglicht. Vgl. G.Tolkemitt, a.a.O., S.12/13.

205 Vgl. G. Tolkemitt, a.a.O., S. 85.

206 Vgl. J.E.Meade: Is the National Debt a Burden? in: J.M.Ferguson: Public Debt and Future Generations, a.a.O., S.20. In einer die Staatsauffassung pointierenden Art kommt dies bei Donner zum Ausdruck: "In seiner Eigenschaft als Besitzer von Staatspapieren wird das Volk Gläubiger des Staates, also seiner selbst, und ebenso bringt es in seiner Eigenschaft als Steuerzahler die für die Bedienung seiner Kapitalansprüche gegen den Staat erforderlichen Mittel selbst auf." O.Donner: Die Grenzen der Staatsverschuldung, in: Weltwirtschaftliches Archiv, Band 56, 1942, S.213.

207 Ein relativer Wachstumseffekt ist in jedem Fall zu erwarten, ein absoluter nur bei geringerer Produktivität der Staatsausgaben als der der alternativen privaten Verwendung.

208 Auf die Problematik einer solchen Quotenorientierung wurde schon hingewiesen.

209 Auch Ricardos Ansatz stellt eine Differentialbetrachtung dar, doch sind diese Überlegungen von genereller Bedeutung für intertemporale Effekte der Staatsverschuldung. D.Ricardo: Principles of Political Economy 
and Taxation (hrsgg. von R.M.Hartwell) London 1971, Kapitel 17.

210 Da hier nur auf die maßgeblichen Gründe für die Indifferenz eingegangen werden soll, sei von Fragen der mit der individuellen Lebensdauer zusammenhängenden Komplikationen abgesehen.

211 Vgl. O.Gandenberger: Zur Rationalität der öffentlichen Kreditnahme. Verhaltenshypothesen und normative Konsequenzen, in: Finanzarchiv, NF Band 30, 1971, S. 369-391.

212 D.Ricardo, a.a.O., S. 254.

213 A.Puviani: Die Illusion in der Finanzwirtschaft, Berlin 1960, S. 53. Vgl. a. J.M.Buchanan: Public Debt, Cost Theory and Fiscal Illusion, in: J.M.Ferguson: Public Debt and Future Generations, a.a.O., S. 156 u. ders.: Public Finance in Democratic Process, Chapel Hill 1967, S. $132 / 133$.

214 O.Gandenberger: Zur Rationalität der öffentlichen Kreditnahme, a.a.O., S. 378 .

215 Diese Aussage kann auch beinhalten, daß es bestimmten Individuen überhaupt unmöglich ist, Kredite zu erhalten.

216 So U.Schmah: Der Einkommensredistributionseffekt der Anleihefinanzierung bei unterschiedlichen Zeitpräferenzen, in: Finanzarchiv, NF Band 26, 1967, S.232-237 und A.A.Walters: How to Make a Benefit of the Burden of National Debt, in: National Tax Journal, Vol. XX, 1967, S. 316-319.

217 Zumindest nach Walters gehen Überlegungen bezüglich der Zeitpräferenzen in den Bereich der Verteilungspolitik ein, da dieser bei bestimmten Parteien aus obengenannten Gründen Präferenzen für eine Anleihefinanzierung festzustellen glaubt. Vgl. A.A.Walters, a.a.O., S.318. Es wäre allerdings auch die Alternativhypothese möglich, daß die höhere Bequemlichkeit (Lautlosigkeit) dieser Finanzierungsart das eigentliche Motiv darstellt.

218 Vgl. zu diesem auch als "Tullock's Fallacy" bekannten Sachverhalt J.M. Buchanan: Public Finance in Democratic Process, a.a.O., S.257-261 und O.Gandenberger: Zur Rationalität der öffentlichen Kreditnahme, a.a.O., S. $387 / 388$.

219 Daß es sich dabei meist um die Verteilung von Zuwächsen handelt, die absoluten Verteilungspositionen einzelner Gruppen also nicht tangiert werden müssen, entkräftet diese Argumentation nicht.

220 Der Begriff einer intrapersonellen (Um)Verteilung scheint deswegen gerechtfertigt, da es sich primär um Umverteilungen des Lebenseinkommens handelt. 
221 Vgl. dazu die konsequent individualistischen Schlußfolgerungen Gandenbergers zum subsidiären Einsatz der Steuerfinanzierung, die die intertemporale Verteilung $\mathrm{zu}$ beeinflussen habe. Allerdings gilt es bei der Gesamtbeurteilung auch die Informationsproblematik $\mathrm{zu}$ berücksichtigen. "...so ergibt sich die verwirrende Feststellung, daß die staatliche Verschuldung die Rationalität der zeitlichen Konsumentscheidungen zugleich fördert und hemmt." O.Gandenberger, a.a.O., S.389 u. 391.

222 Es sollen hier das grundlegende Werk und die neueren Veröffentlichungen angeführt werden, zu einem Gesamtiberblick siehe Literaturverzeichnis: J.M.Buchanan: Public Principles of Public Debt, Homewood 1958; aus neuerer Zeit Buchanan u. R.E.Wagner: Public Debt in a Democratic Society, Washington 1967; J.M.Buchanan: Cost and Choice. An Inquiry in Economic Theory, Chicago 1969; J.M.Buchanan u. M.R. Flowers: The Public Finances, 4. Auflage, Homewood 1975.

223 Vgl. etwa J.M.Buchanan: Concerning Future Generations in: J.M. Ferguson (Hrsg.): Public Debt and Future Generations, a.a.O., S.57/58. In diesem Sinne äußert sich auch Gandenberger, der bestreitet, daß diesem Lastbegriff für eine marktwirtschaftliche Ordnung irgendeine Bedeutung zukommen könne. O.Gandenberger: Ist es möglich, durch die Aufnahme von öffentlichem Kredit die Last einer öffentlichen Aus gabe in die Zukunft zu verschieben? in: Jahrbuch für Sozialwissenschaft, Band 21, 1970, S. 98 .

224 J. Tobin: "The Burden of the Public Debt: A Review Article" in: The Journal of Finance, Band 20, 1965, S.679.

225 Auf die u.U. durch Buchanan beeinflußte Modifikation der einfachen Aussagen der Neuen Orthodoxie zum Wachstumsansatz wurde schon verwiesen.

226 Vgl. zur Bedeutung dieser Annahme: "To begin with, it cannot be overemphasized that this discussion relates to debt burden in an economy of the classical type...". R.A.Musgrave: Public Debt and Future Generations (Besprechung des Sammelbandes von Ferguson d.V.) in: American Economic Review, Band 55, 1965, S. 1226.

227 Auf die methodische Schwäche dieses Vorgehens, das alle Phänomene, die mit Liquiditätseffekten verbunden sind, hinwegdefiniert, wurde oben schon eingegangen. Diese enge Begriffsfassung einer "real debt" kommt in folgendem Zitat zum Ausdruck: "Broadly speaking, there are only two means of financing budget deficits. One is by borrowing; that is, by issuing interest-bearing debt obligations in exchange for current command over purchasing power. The second is by printing money; that is, by issuing or creating noninterest bearing money or currency which becomes acceptable directly as purchasing power." J.M.Buchanan: Public Finance in Democratic Process, Chapel Hill 1967, S.99. 
228 Vgl. O.Gandenberger: Intertemporale Verteilungswirkungen der Staatsverschuldung, a.a.O., S.198, FN 21).

229 Vgl. K. Meister, a.a.O., S.215/216 und 218.

230 Auf die Bedeutung dieser Annahme verweisen S.C.Shoup, a.a.O., S.467 und E.J.Mishan, a.a.O., S.189, FN 6).

231 "Nowhere has the elemental confusion in cost theory been more in evidence than in the sometimes acrimonious discussion of public-debt incidence." J.M.Buchanan: Cost and Choise Chicago 1960, S.62

232 So distanziert er sich von seinen "Public Principles of Public Debt" und dem im Ferguson-Sammelband erschienenen Aufsatz "Public Debt, Cost Theory, and the Fiscal Illusion". Vgl. ebd., S.47 und S.63, FN 5). Vgl. dazu auch die Beurteilung bei E.J.Mishan: The Burden of the Public Debt: A.Rejoinder, in: Journal of Political Economy, Band 72, 1964, S.491 und R.A.Musgrave: Public Debt and Future Generations, a.a.O., S.1227.

233 K. Kiepe: Die Thesen J.M.Buchanans zur Last der Staatsverschuldung. Diss. Münster 1970. Kiepe liefert zwar einen Überblick über die Begriffswandlung in den Arbeiten Buchanans, berücksichtigt aber nicht die den Kostenbegriff klärende Arbeit "Cost and Choice". So erkennt Kiepe nie den Ausgangspunkt Buchanans als der subjektivistischen Wertlehre zugehörig.

234 "The predictive science of economics", ebd. S.38.

235 Ebd. S. 25.

236 Vgl. zur Darstellung dieser Kostenarten J.M.Buchanan: Cost and Choice, a.a.O., S. $44 / 45$.

237 "The choice-influenced costs of debt-financed projects, are borne exclusively in periods subsequent to decision. These actual payments, which may also be measured in money, may reduce the utilities of others than those who participate in the decision." J.M.Buchanan: Cost and Choice, a.a.O., S. 64 .

238 Vgl. J.M.Buchanan: Public Principles of Public Debt, a.a.O., S.96.

239 Es ist hier noch einmal an die stringenten Annahmen Buchanans zu erinnern, wonach der Staatsverschuldung automatisch ein entsprechender freiwilliger Konsumverzicht entspricht.

240 "Who gives up command over the real resources that are secured for public use under debt financing? The obvious answer is those who purchase the debt instruments from the treasury. These bond-purchasers are not at all concerned about the decision to issue debt; their choice is simply whether to purchase debt or to purchase privately available investment or consumption goods. These bond purchasers surely don't participate in the fiscal choice as such. They 
cannot be said to bear the 'cost' of the public goods that the debt issue finances." J.M.Buchanan: Cost and Choice, Chicago 1969, S.63.

$241 "$ "... only the decrease in net worth of the tax-payers may be attributed to the fiscal operation under consideration." J.M.Buchanan: Public Principles of Public Debt, a.a.O., S. 54 und S. 57/58.

242 So etwa E.R.Rolph und G.F.Break: Public Finance, New York 1961, S.547, J.Tobin: The Burden of the Public Debt: A Review Article, a.a.O., S.680, K.Kiepe, a.a.O., S.92, O.Gandenberger: Intertemporale Verteilungswirkungen, a.a.O., S.201.

243 "The answer to this question is that the loss in utility suffered by disappointed borrowers cannot legitimately be defined as a part of the real-debt burden. This loss in utility is offset by some gain in utility enjoyed by lenders. These gains and losses represent secondary repercussions of the government's action in issuing debt". J.M.Buchanan: Public Principles of Public Debt, a.a.O., S.93. Auch diese, gewissermaßen unkritische Einstellung gegenüber dem Marktgeschehen ist $u . U$. mit Buchanans subjektivistischen Wertvorstellungen erklärbar.

244 Mit seiner Begründung, daß dem privaten Bereich trotz dieser Sekundäreffekte eine unveränderte Gütermenge zur Verfügung steht, die Anleihezeichnung erfolgte ja freiwillig, rückt Buchanan in erstaunliche Nähe des Ansatzes der Neuen Orthodoxie, da auch er damit nur auf die Dichotomie zwischen privatem und öffentlichem Sektor abstellt, strukturelle Effekte innerhalb des privaten Bereichs aber vernachlässigt. Vgl. J.M. Buchanan, ebd.

245 In seinen zahlreichen Veröffentlichungen betont Buchanan immer wieder, daß er nur die "echte" Staatsverschuldung, nicht aber eine reine Geldschöpfung untersuche. "The confusion between money issue, which does not involve any shifting of cost forward in time, and debt issue, which does involve such a shifting, has persisted throughout the post-Keynesian discussion of fiscal policy." J.M.Buchanan und R.E.Wagner: Public Debt in a Democratic Society, Washington 1967, S. 59.

246 Vgl. J.M.Buchanan: Public Principles of Public Debt, a.a.O., S.99/100 und S.135-144.

247 W.Wittmann: Einführung in die Finanzwissenschaft. III. Teil, Stuttgart 1972, S. 16.

248 Ähnliche Formulierungen finden sich bei H.Zimmermann und K. -D.Henke: Einführung in die Finanzwissenschaft, München 1975, S.308. M.E.Kamp, W.Schönebeck, D.Smolinski, H.Weiler: Öffentliche Finanzwirtschaft, Köln 1975, S.213. G.Schmölders: Finanzpolitik, 3. Auflage, Berlin/ Heidelberg/New York 1970, S.419/420. 
249 "What is sought is the incidence, the pattern of distribution, interpersonally and intertemporally of the cost, which must exist in any of the models under consideration". J.M.Buchanan: Confessions of a Burden Monger, in: The Journal of Political Economy, Bd. LXXII, 1964, S. 486.

250 Vgl. A.T. Peacock: The Rehabilitation of Classical Debt Theory, in: Economica, Band 26, 1959, S. 164.

251 Trotz der Verwendung des Begriffs der intergenerativen Effekte stellt Buchanan nur auf solche intertemporaler Art ab: "I shall not be concerned as to whether a public debt burden is transfered to our children or grand-children as such. I shall be concerned with whether or not the debt burden can be postponed." J.M.Buchanan: Public Principles of Public Debt, a.a.O., S. 34 .

252 W.G.Bowen, R.G.Davis und D.H.Kopf: The Public Debt: A Burden on Future Generations? in: The American Economic Review, Band L, 1960, S.67ff. wiederabgedruckt in: J.M.Ferguson: Public Debt and Future Generations, a.a.O., S.67-74.

253 Wenn Bowen-Davis-Kopf auch auf die individuelle Verschiebbarkeit abstellen, führen sie diese Überlegungen mit einem aggregierten Modell aus, was in der Literatur zur falschen Beurteilung führte, daß ihre Betrachtung den makroökonomischen Ansätzen zuzurechnen sei. So etwa A.P.Lerner: The Burden of Debt, in: J.M.Ferguson: Public Debt and Future Generations, a.a.O., S.92 und W.Wittmann: Einfuhrung in die Finanzwissenschaft, Teil III, Stuttgart 1972, S.16.

254 Allerdings wird dieser bei Buchanan zentrale Effekt hier zu einem sekundären, worin aber nur das andersartige Untersuchungsziel zum Ausdruck kommt. Vgl. E.J.Mishan: How to Make a Burden of the Public Debt, a.a.O., S.195, FN 12).

$255 \mathrm{Vgl}$. die Hinweise bei W.Vickrey: The Burden of the Public Debt: Comment in: Public Debt and Future Generations, a.a.O., S. 79 und J.R.Elliott: The Burden of the Public Debt: Comment,in: J.M.Ferguson, a.a.O., S.84. Allerdings muß wieder darauf verwiesen werden, daß Bowen-Davis-Kopf lediglich die theoretische Möglichkeit einer solchen Verschiebbarkeit nachweisen wollen, dies aber nicht als realistische Verhaltensannahme interpretieren.

256 J.G.Head: The Theory of Debt Incidence, in: Rivista di Diritto Finanziario e Scienza delle Finanze, Band 26, 1967, S.175ff. Die folgende Zusammenfassung lehnt sich an diesen Aufsatz an.

257 "In our analysis of debt incidence we shall accordingly define the burden concept in terms of a reduction in potential lifetime consumption, including therefore not only ownconsumption in the narrow sense but also gifts and overlife savings, all suitably discounted to some convenient date." J.G.Head, a.a.O., S.181. 
258 So werden die Einflüsse unterschiedlicher Informationsniveaus nicht verfolgt, sondern vom Fall der vollkommenen Information ausgegangen.

259 "The future taxes falling on their prospective heirs must similarly be regarded as equivalent to a reduction in bequests". J.G.Head, a.a.O., S.189. Head vergleicht die Wirkung der so abdiskontierten Steueranteile künftiger Generationen explizit mit denen einer Erbschaftsteuer ebd. FN 38).

260 Was nicht ausschließt, daß eine solche Identität zustande kommt.

261 Allerdings setzt diese Verhaltensannahme die Fähigkeit zu anderweitiger Vermögensbildung voraus.

262 Durch jede nichterfolgte Antizipation wird die Belastung der kommenden Generation tendenziell größer.

263 So erhebt Tobin gegenüber Buchanan den Vorwurf: "By primary analysis he means simple Internal Revenue accounting: Who paid taxes when..." J. Tobin: Reply in: Journal of Finance, Band 21, 1966, S. 577.

264 In individueller Sicht ermöglicht jeder Vermögensbestand, damit auch staatliche Papiere, durch Veräußerung Rückgriffsmöglichkeiten auf zusätzlichen Konsum.

265 So wies Tullock schon zu Beginn der Diskussion auf den ergänzenden Charakter der mikro- und makroökonomischen Ansätze hin. G. Tullock: Public Debt - Who Bears the Burden? in: J.M.Ferguson: Public Debt and Future Generation, a.a.O., S.101.

266 Vgl. T.Scitovsky: The Burden of the Public Debt: Comment, in: J.M. Ferguson: Public Debt and Future Generations, a.a.O., S.82/83.

267 So in J.M.Buchanan: The Icons of the Public Debt, in: Journal of Finance, Band 21, 1966, S. 545 .

268 Vgl, zur folgenden Darstellung J.M.Buchanan: Cost and Choice, a.a.O., S. $64 / 65$.

269 "The decision of a prospective bond-purchaser is of course relevant to the rate of private capital formation, but this is not the same decision as that of the prospective bond-sellers. If the bond purchaser draws down private investment, he does impose a 'burden' on his heirs in future periods, and the recognition of this will be the obstacle to his choice." J.M.Buchanan: Cost and Choice, a.a.O., S.65. Eine ähnliche Argumentation klingt bei Meister an.

Meister, a.a.O., S.82.

270 Vgl. hierzu Phelps: "... it is not possible for the public to express its preferences for growth through the market mechanism." E.S. Phelps: Fiscal Neutrality toward Economic Growth, New York, 1965, S.13. 
271 Dies klingt auch bei Buchanan an: "The emphasis on the capitalformation aspects of public debt seems to arise from a confusion of the results of not one, but two decisions, and the calculus of not one, but two sets of decisionsmakers." J.M.Buchanan: Cost and Choice, a.a.O., S. 65 .

272 Allerdings ist auch bei Bowen-Davis-Kopf Voraussetzung für den Nachholkonsum, daß die jeweils jüngere Generation zusätzliche Ersparnisse bildet, da andernfalls Preisniveaueffekte oder Wachstumseinbußen die Folge wären (c.p.).

273 Vgl. Diskussionsbeitrag von W.Albers in: H.Giersch (Hrsg.): Fiscal Policy and Demand Management, Tübingen 1973, S.123.

274 Hier ist wieder an die Quotenbetrachtung zu erinnern, die absoluten Ansprüche können auch bei konstanter Quote zunehmen.

275 Daß in einer solchen Situation auf Zentralbankkredite zurückgegriffen werden sollte, wird von vielen an der Lastdebatte Beteiligten gefordert. So etwa J.M.Buchanan: Public Principles of Public Debt, a.a.O., S.126.

276 Diese Überlegungen entsprechen im Grundgedanken der Vorstellung Oberhausers von der asymmetrischen Konjunkturpolitik. Vgl. A. Oberhauser: Stabilitätspolitik bei steigender Staatsquote, Göttingen 1975, S. 77ff.

Ähnliche Gedanken klingen an bei O. Eckstein: Public Finance, 3. Auflage, Englewood Cliffs, 1973, S.93.

277 Solche unmittelbar dem Aggregate Investment Approach verbundenen Überlegungen für die Situation der Unterbeschäftigung finden sich bei F. Modigliani: Long-Run Implications ... a.a. O., S.137.

278 Wobei natürlich nicht jede expansive Politik mittels Staatsausgaben betrieben werden muß, der private Sektor kann auch unmittelbar angeregt werden.

279 Wäre lediglich ein Niveaueffekt festzustellen, würde dies nur das Verhältnis zwischen Vermögensbesitzern und Nicht-Vermögensbesitzern berühren.

280 W. Engels, H.Sablotny und D.Zickler: Das Volksvermögen, Frankfurt 1974, S.95-99. S. hierzu auch O. Pfleiderer: Realvermögen und Geldvermögen unter dem Einfluß der Inflation, in: W. Albers (Hrsg.): Öffentliche Finanzwirtschaft und Verteilung II, Schriften des Vereins für Socialpolitik, NF Band 75, II, Berlin 1974, S. 26 und A. Oberhauser: Entwicklungstendenzen und Bestimmungsfaktoren der Vermögensbildung und Vermögensverteilung in der Bundesrepublik Deutschland, in: G. Bruns u. K.Häuser (Hrsg.): Vermögensbildung, Vermögensverteilung und Kapitalmarkt, Frankfurt 1974, S.42. 
281 H.Willgerodt, K. Bartel u. U.Schillert: Vermögen für alle. Probleme der Bildung, Verteilung und Werterhaltung des Vermögens in der Marktwirtschaft, Düsseldorf-Wien 1971, insbes. S.150-152. K. Bartel: Öffentliche Schuld und privates Vermögen, in: Ordo, Band 23, 1972, S.261. In ähnlichem Sinne auch B.Molitor: Öffentliche Armut - privater Reichtum, in: Ordo, Band 24, 1973, S.154/155. K. Bartel: Öffentliche Infrastruktur oder privates Vermögen. Die vermögenswirksame Schuldenpolitik als Mittel zur Lösung eines Zielkonflikts, Berlin 1975

282 Vgl. H.Haller: Neue Motivation der Staatsverschuldung, in: H.Haller $u$. H.C.Recktenwald (Hrsg.): Finanz- und Geldpolitik im Umbruch, Mainz 1969, S.324ff. Haller sieht allerdings diese Situation als eine unter möglichen anderen an.

283 Vgl. O.Gandenberger: Öffentlicher Kredit und Einkommensverteilung, a.a.O., S. 10 und S. 14 .

284 Hier ist allerdings darauf zu verweisen, daß u.U. gegenläufige Effekte (etwa Ertragsänderungen) diese Einflüsse konterkarieren können.

285 Größere Bedeutung wäre ihm erst dann zuzusprechen, wenn das reale Vermögen als Argument in die Konsumfunktion einginge, dann wären auch temporäre Wertänderungen zu beachten. Vgl. J.Siebke u. M.Willms: Geldpolitik und Vermögenseffekte, in: S.Klatt u. M.Willms (Hrsg.): Strukturwandel und makroökonomische Steuerung, Festschrift für Fritz Voigt, Berlin 1975, S.330.

286 Zudem sind die Verteilungsgewinne der Individuen zu berücksichtigen, die durch Verzicht auf Kassenhaltung realisiert werden. Vgl. K.H.Hansmeyer: Der öffentliche Kredit, a.a.O., S.109. H.Zimmermann u. K. -D. Henke, a.a.O., S.201.

287 Hier ist an den Vergleich der Keynesschen Verteilungsgleichung mit dem Kaldor-Modell zu erinnern (s.o. S.47). Während im Fall der Verteilungsgleichung, für den in verteilungstheoretischer Interpretation eine marginale Sparneigung der Unternehmer von eins unterstellt wurde, auch eine summenmäßige Identität zwischen Einkommenserhöhung und zusätzlicher Vermögensbildung festzustellen ist, bestimmt sich dieses Verhältnis bei Kaldor nach Maßgabe des Sensitivitätskoeffizienten.

288 Vgl. G. L. Bach und S. Ando: The Redistributional Effects of Inflation, in: The Review of Economics and Statistics, Band 39, 1957, S.2.

289 In diesem Sinne G.L.Bach und J.B.Stephenson: Inflation and the Redis tribution of Wealth, in: The Review of Economics and Statistics, Band 56, 1974, S.2ff. Vgl. hierzu den methodischen Einwand Jecks, der auf die Bestimmungsschwierigkeit hinweist, da die Vergleichsgröße die des fiktiven Zinssatzes einer Situation ohne Inflation sein müßte. A.Jeck: Wer gewinnt, wer verliert bei einer Inflation? in: J.Schlemmr (Hrsg.): Enteignung durch Inflation, München 1972, S.92ff und R.Hauser: Geld- 
wertgesicherte Anlagemöglichkeiten als neues sozialpolitisches Instrument, in: Finanzarchiv, NF Band 34, 1976, S.461.

$290 \mathrm{Vgl}$. M.Ziercke: Inflation und Einkommensverteilung in der Bundesrepublik von 1950 bis 1969, in: Jahrbuch für Sozialwissenschaft, Band 22, 1971, S. 143; vgl, auch die positive Stellungnahme bei L.Wicke: Schuldnergewinne und Gläubigerverluste während einer schleichenden Inflation, in: Zeitschrift für Wirtschafts - und Sozialwissenschaften, 92. Jhg., 1972, II. Halbband, S. 563 und 580 .

291 So bei O. Pfleiderer: Realvermögen und Geldvermögen unter dem Einfluß der Inflation, a.a.O., S.9ff.

292 Stellt man dabei nur auf den privaten Sektor ab, so nehmen die staatlichen Papiere eine Ausnahmestellung ein. Sofern sich die Wirtschaftssubjekte durch die ausstehenden Zins- und Tilgungsverpflichtungen nicht in einer Schuldnerposition sehen, ergibt sich hier keine Saldierung. Da wir eine solche Abdiskontierung aber auch bei den Fragen der intertemporalen Verteilung als nicht realitätsnah verwarfen, soll hier von der Nettoposition des Staats ausgegangen werden. Dies stellt für die Beurteilung der Gläubiger-Schuldner-Hypothese keinen zentralen Punkt dar, da auf die gesamten Nominalvermögensbestände abgestellt werden muß, wohl aber für die Beantwortung der Frage nach der Inflationsschädigung des Staates. Nimmt dieser, wie hier unterstellt, eine Schuldnerposition (durch die Staatsverschuldung) ein, so zählt er zumindest in diesem Punkt zu den Inflationsgewinnern. Damit lehnen wir auch die in der Literatur zu findenden Überlegungen $a b$, die solche Inflationsgewinne wieder den Zensiten zurechnen. So etwa H.Scherf: Inflation und Einkommensverteilung, in: Weltwirtschaftliches Archiv, Band 108, 1968, S.268. A.Jeck: Wer gewinnt, wer verliert bei einer Inflation? a.a.O., S.97/98.

293 Vgl. W. Mückl u. R.Hauser: Die Wirkungen der Inflation auf die Einkommens - und Vermögensverteilung, Schriften der Kommission für wirtschaftlichen und sozialen Wandel 60, Göttingen 1975, S.20. Ähnlich L.Wicke: Vermögensverteilung und schleichende Inflation, Meisenheim am Glan 1975 , S. 154 .

294 Vgl. zu den Problemen der Vermögensstatistik A.Hansen: Die Einkommensund Vermögensverteilung in der Bundesrepublik Deutschland aus statistischer Sicht, in: W. Albers (Hrsg.): Öffentliche Finanzwirtschaft und Verteilung I, Schriften des Vereins für Socialpolitik, NF Band 75/I, Berlin 1974, S.49-68. Deutsche Bundesbank (Hrsg.): Entwicklung des Geldvermögens und der Verpflichtungen von 1950 bis 1971, in: Monatsberichte der Deutschen Bundesbank, 25. Jg., 1973, Heft 3, S.26/27. Zur Gegenposition, die den Staat in der Schuldnerposition sieht vgl. G. L. Bach u. J.B.Stephenson, a.a.O., S. 4 .

295 Vgl. Engels/Sablotny/Zickler, a.a.O., S.133, hier insbesondere relevant dritter Teil: Vermögensbildung und Vermögensbestände bei den Privathaushalten, S. 133-168. 
296 Vgl. zur chronologisch vollständigen Übersicht W. Engels, H.Sablotny u. D.Zickler, a.a.O., S.142, Tab. 37 und 38 .

297 Diese Größe umfaßt in der von Engels und Mitautoren gebrauchten $\mathrm{Ab}$ grenzung neben Bargeld und Sichtguthaben die Spareinlagen und festverzinsliche Wertpapiere, nicht aber Aktien und Investmentzertifikate. Vgl. Engels u.a., a.a.O., S.138, FN 55).

298 Zur Illustration seien noch die Absolutzahlen des Geldvermögens hinzugefügt: dieses betrug 1970 bei den Selbständigen 116,4 Mrd. DM, bei den Unselbständigen hingegen 278,6 Mrd. DM. Letztere besitzen also $70 \%$ des gesamten Geldvermögens privater Haushalte.

299 W.Engels, H.Sablotny u. D.Zickler, a.a. O., S.144.

300 Vgl. W.Krelle: Vermögensverteilung: Zahlen, Empfehlungen, Absichtserklärungen, in: Wirtschaftswoche, Band 26, 1972, Heft 39, S.73/74.

301 W.Engels, H.Sablotny u. D.Zickler, a.a.O., S.146.

302 Vgl. L.Wicke: Vermögensverteilung und schleichende Inflation, a.a.O., S.252/253. Die Bedeutung von Haus - und Grundbesitz hebt auch Hauser hervor, da damit häufig eine Schuldnerposition verbunden ist. Vgl. $R$. Hauser: Geldwertgesicherte Anlagemöglichkeiten als neues sozialpolitisches Instrument, a.a.O., S.467. Eine differenzierende Betrachtung nach dem Alter der Haushalte findet sich bei G.L.Bach und J.B. Stephenson, a.a.O., S.13 sowie M.Ziercke, a.a.O., S.140.

303 Als Erklärungsmotiv dieser Anlageform gibt Pfleiderer an, daß "vielfach 'faute de mieux' Geldvermögen gebildet wird". O.Pfleiderer, a.a.O., S. 12 .

Vgl. auch H.Schlesinger: Vermögensbildung und Vermögensverteilung in der Bundesrepublik Deutschland, in: G.Bruns u. K.Häuser (Hrsg.): Vermögensbildung, Vermögensverteilung und Kapitalmarkt, Frankfurt 1974, S. 11 .

304 So fordert Hauser aufgrund der fehlenden Alternativen für die unteren Einkommensschichten eine geldwertgesicherte Anlagemöglichkeit. Vgl. R.Hauser: Geldwertgesicherte Anlagemöglichkeiten als neues sozialpolitisches Instrument, a.a. O., S. 469ff.

305 Die Auseinandersetzung mit der klassischen Schuldtheorie hat gezeigt, da $\beta$ auch bei modellimmanenter Argumentation strukturelle Verteilungs effekte bei der Schuldaufnahme zu beobachten sind.

306 Daß nämlich in dem Maße, in dem die staatliche Kreditnachfrage nicht durch freiwillige Mehrersparnis alimentierbar ist, die privaten Investitionen zurückgedrängt werden.

307 A.T. Peacock: The Rehabilitation of Classical Debt Theory, in: Economica, Band 26, 1959, S.166. Dabei verkörpert in Peacocks Metapher der kleine Junge die staatliche Verschuldung, der Stein die inflationären Wirkungen. 
zu $\left.c_{1}\right)$ Fall I: Steuerzahler:Arbeitnehmer Zinsempfänger:Unternehmer

1. Bestimmung der Quoten der Primärverteilung

Für die Ersparnisbildung der Unternehmer bzw. Arbeitnehmer ergibt sich bei Berücksichtigung der jeweiligen Verhaltensfunktionen und der Annahmen $\mathrm{Z}_{\mathrm{nu}}=\mathrm{O}, \mathrm{T}_{\mathrm{u}}=\mathrm{O}$ :

$$
\begin{array}{ll}
\text { IV.13. a } & S_{n u}=s_{n u}\left(1-t_{n u}\right) Y_{n u} \\
\text { IV.14. a } & S_{u}=s_{u}\left(Y_{u}+Z_{u}\right)
\end{array}
$$

Ferner gilt nach IV.8. -IV.11.: $\mathrm{Z}_{\mathrm{u}}=\mathrm{t}_{\mathrm{nu}}\left(\mathrm{Y}-\mathrm{Y}_{\mathrm{u}}\right)$.

Werden diese Ausdrücke unter Berücksichtigung von IV.1. in IV.15. eingesetzt, so ergibt sich:

$$
I=s_{n u}\left(1-t_{n u}\right) Y-s_{n u}\left(1-t_{n u}\right) Y_{u}+s_{u} Y_{u}+s_{u} t_{n u} Y-s_{n u} t_{n u} Y_{u}
$$

und daraus nach $\mathrm{Y}_{\mathrm{u}} / \mathrm{Y}$ aufgelöst IV. 16 .

Analog zu obigem Vorgehen ergibt sich bei der Bestimmung der Arbeitnehmereinkommen:

$$
\begin{aligned}
& I=s_{n u}\left(1-t_{n u}\right) Y_{n u}+s_{u} Y-s_{u} Y_{n u}+s_{u} t_{n u} Y_{n u} . \\
& \frac{Y n u}{Y}=\frac{s_{u}-I / Y}{s_{u}\left(1-t_{n u}\right)-s_{n u}\left(1-t_{n u}\right)}=\frac{s_{u}-I / Y}{s_{u}-\left[s_{n u}+\left(s_{u}-s_{n u}\right) t_{n u}\right]}
\end{aligned}
$$

Komplementär zur Verringerung der Quote der Unternehmereinkommen ergibt sich für die Primärverteilung der Arbeitnehmer eine Verbesserung.

2. Bestimmung der Quoten der Sekundärverteilung

$$
\text { IV.18. } \quad \frac{Y_{u}^{v}}{Y}=\frac{Y u}{Y}\left(1-t_{n u}\right)+t_{n u}
$$

Ersetzt man $Y_{u} / Y$ durch Gleichung IV.17., so sind folgende Umformungen im Zähler von IV.18. zu beachten (der Nenner von IV.17. bleibt von diesen Umformungen unberührt): 
Zähler von IV.18. nach Substitution von $Y_{u} / Y$ durch IV.17. = $s_{n u}-s_{n u} t_{n u}+t_{n u} s_{u}-t_{n u} s_{n u}-t_{n u} s_{u} t_{n u}+t_{n u} s_{n u} t_{n u}-I / Y+I / Y t_{n u}+t_{n u} s_{n u}$ $+t_{n u} s_{u} t_{n u}-t_{n u} s_{n u} t_{n u}-s_{u} t_{n u}=s_{n u}-t_{n u} s_{n u}-I / Y+t_{n u} I / Y$

Für IV.18. erhält man dann:

$\frac{Y_{u}^{v}}{Y}=\frac{s_{n u}-I / Y\left(1-t_{n u}\right)-t_{n u} s_{n u}}{s_{n u}+t_{n u}\left(s_{u}-s_{n u}\right)-s_{u}}=\frac{\left(1-t_{n u}\right)\left(s_{n u}-I / Y\right)}{\left(1-t_{n u}\right)\left(s_{n u}-s_{u}\right)}$

Auch für die Quote der verfügbaren Einkommen der Arbeitnehmer ergibt sich durch Multiplikation obigen Ausdrucks für $\mathrm{Y}_{n u} / \mathrm{Y}$ mit dem Faktor $\left(1-\mathrm{t}_{\mathrm{nu}}\right)^{\prime}$ eine Konstanz.

Anhang II

zu $c_{2}$ ) Fall II $\begin{gathered}\text { Steuerzahler:Unternehmer } \\ \text { Zinsempfänger:Arbeitnehmer }\end{gathered}$

Auch hier geht man von Gleichung IV.13 u. IV.14 aus, wobei nun $\mathrm{Z}_{\mathrm{u}}=\mathrm{O}$ und $\mathrm{T}_{\mathrm{nu}}=\mathrm{O}$ gilt:

$$
\begin{aligned}
& s_{n u}=s_{n u}\left(Y_{n u}+Z_{n u}\right) \\
& s_{u}=s_{u}\left(1-t_{u}\right) Y_{u}
\end{aligned}
$$

Aus IV.8. -IV. 12. resultiert $Z_{n u}=t_{u} Y_{u}$. Bei Substitution von $Z_{u}$ durch diesen Ausdruck und unter Verwendung von IV.1 ergibt sich:

$$
I=s_{n u} Y-s_{n u} Y_{u}+s_{n u} t_{u} Y_{u}+s_{u}\left(1-t_{u}\right) Y_{u}
$$

und daraus nach $Y_{u} / Y$ aufgelöst Gleichung IV.20.. 
$z \mathrm{c}_{3}$ ) Fall III: Verallgemeinerung

In diesem allgemeinen Fall können beide Gruppen sowohl Aufbringer als auch Empfänger staatlicher Zinszahlungen sein, wobei die Verteilung der Papiere durch den Parameter $\alpha$ gekennzeichnet ist.

Die Ersparnisbildung der beiden Gruppen ist analog zu oben:

$$
S_{u}=s_{u}\left(1-t_{u}\right) Y_{u}+s_{u} Z_{u}
$$

$$
s_{n u}=s_{n u}\left(1-t_{n u}\right) Y_{n u}+s_{n u} Z_{n u}
$$

Ferner ist nach IV.8. -IV.12. und IV.22:

$$
Z_{u}=\alpha\left(t_{n u} Y_{n u}+t_{u} Y_{u}\right) \text { und } Z_{n u}=(1-\alpha)\left(t_{n u} Y_{n u}+t_{u} Y_{u}\right)
$$

Diese Ausdrücke für die Zinszahlungen werden unter Berücksichtigung von IV.1. in IV.15. eingesetzt:

$$
I=\left[s_{n u}\left(1-t_{n u}\right)+s_{n u}(1-\alpha) t_{n u}+s_{u} \alpha t_{n u}\right]\left(Y-Y_{u}\right)+\left[s_{u}\left(1-t_{u}\right)+s_{u} \alpha t_{u}+s_{n u}(1-\alpha) t_{u}\right] Y_{u}
$$

Auflösung nach $\mathrm{Y}_{\mathrm{u}} / \mathrm{Y}$ :

$$
\begin{aligned}
& \frac{Y_{u}}{Y}=\frac{\left[s_{n u}\left(1-t_{n u}\right)+s_{n u}(1-\alpha) t_{n u}+s_{u} \alpha t_{n u}\right]-}{\left[s_{n u}\left(1-t_{n u}\right)+s_{n u}(1-\alpha) t_{n u}+s_{u} \alpha t_{n u}\right]-\left[s_{u}\left(1-t_{u}\right)+s_{u} \alpha t_{u}+s_{n u}(1-\alpha) t_{u}\right]} \\
& =\frac{s_{n u}-s_{n u} t_{n u}+s_{n u} t_{n u}-s_{n u} \alpha t_{n u}+s_{u} \alpha t_{n u}-I / Y}{s_{n u}-s_{n u} t_{n u}+s_{n u} t_{n u}-s_{n u} \alpha t_{n u}+s_{u} \alpha t_{n u}-s_{u}+s_{u} t_{u}-s_{u} \alpha t_{u}-s_{n u} t_{n u}+s_{n u} \alpha t_{u}} \\
& =\frac{s_{n u}+\alpha\left(s_{u}-s_{n u}\right) t_{n u}}{s_{n u}+\alpha\left(s_{u}-s_{n u}\right) t_{n u}}-\frac{1 / Y}{\left[s_{u}-t_{u}\left(s_{u}-s_{n u}\right)+t_{u} \alpha\left(s_{u}-s_{n u}\right)\right]}
\end{aligned}
$$





\section{LITERATURVERZEICHNIS}

ALBERS, W., Staatsverschuldung und Geld- und Kreditpolitik, in: Finanzarchiv, NF Bd. 21, 1961, S.25ff.

ALBERS, W., Die automatische Stabilisierungswirkung der Steuern - Möglichkeiten und Problematik in der Bundesrepublik Deutschland, in: Jahrbücher für Nationalökonomie und Statistik, Bd. 180, 1967, S.99ff.

ALBERS, W., Diskussionsbeitrag, in: H.Giersch (Hrsg.): Fiscal Policy and Demand Management, Tübingen 1973.

ANDEL, N., Probleme der Staatsschuldtilgung, Berlin 1964.

ANDEL, N., Öffentliche Schulden und Lastverteilung, in: Finanzarchiv, NF Bd. 25, 1966, S.334ff.

ANDEL, N., Zur These von den unsozialen Verteilungswirkungen öffentlicher Schulden, in: Public Finance, Bd. 24, 1969, S. $69 \mathrm{ff}$.

ANDERSON, R.W., A Note on Tax Incidence in a Macroeconomic Distribution Model, in: Revista Internationale de Sience Economiche e Commerciale, Bd. 16, 1969, S.1164ff.

ANDERSON, W.H., Financing Modern Government, Boston 1973.

ANDREAE, C.A. und SCHLÖGL, K., Finanzpolitik, Stuttgart 1975.

BACH, G.L. und ANDO, S., The Redistributional Effects of Inflation, in: The Review of Economics and Statistics, Bd. 39, 1957, S.1ff.

BACH, G.L. und STEPHENSON, J.B., Inflation and the Redistribution of Wealth, in: The Review of Economics and Statistics, Bd. 56, 1974, S.1ff.

BARTEL, K., Öffentliche Schuld und privates Vermögen, in: Ordo, Bd. 23, 1972, S. 261ff.

BARTEL, K., Öffentliche Infrastruktur oder privates Vermögen. Die vermögenswirksame Schuldenpolitik als Mittel zur Lösung eines Zielkonflikts, Berlin 1975.

BEYFUSS, J., Gesamtwirtschaftliche Probleme der Staatsverschuldung. Beiträge zur Wirtschafts - und Sozialpolitik 33, Institut der deutschen Wirtschaft, Köln 1976.

BITZ, M., Pläne und Maßnahmen zur Vermögensbildung, Köln 1971.

BLÜMLE, G., Verteilungstheorie und makroökonomische Steuerüberwälzungs lehre, in: Jahrbuch für Sozialwissenschaft, Bd. 18, 1967, S.175ff. 
BLÜMLE, G., Theorie der Einkommensverteilung, Berlin-Heidelberg-New York 1975.

BOMBACH, G., Die verschiedenen Ansätze der Verteilungs-Theorie, in:

E.Schneider (Hrsg.): Einkommens verteilung und technischer Fortschritt, Schriften des Vereins für Socialpolitik, NF Bd. 17, Berlin 1959, S.95ff.

BOMBACH, G., Das Problem der optimalen internationalen Arbeitsteilung bei unterschiedlicher Struktur der Steuersysteme mit einem Anhang von Hajo Riese, Düsseldorf 1962 .

BOMBACH, G., Neue Dimensionen der Lehre von der Einkommensverteilung, Basler Universitäts reden, 66. Heft, Basel 1972.

BOMBACH, G., Die öffentliche Finanzwirtschaft im Wirtschaftskreislauf, in: F. Neumark (Hrsg.): Handbuch der Finanzwissenschaft, Bd. 1, Lieferung 1, 3. gänzlich neubearbeitete Auflage, Tübingen 1975, S. 53ff.

BOWEN, W.G., DAVIS, R.G. und KOPF, D.H., The Public Debt: A Burden on Future Generations? in: The American Economic Review, Bd. L, 1960 , S. $67 \mathrm{ff}$.

BREAK, G.F., The Incidence and Economic Effects of Taxation, in: A.S. Blinder, R.S.Solow, G.F.Break, P.O.Steiner, D. Netzer: The Economics of Public Finance. Studies of Government Finance, Brookings Institution, Washington D.C. 1974, S.119ff.

BRÜMMERHOFF, D., Gesamtwirtschaftliches Rechnungswesen, Köln 1975.

BUCHANAN, J.M., Public Principles of Public Debt, Homewood 1958.

BUCHANAN, J.M., Confessions of a Burden Monger, in: The Journal of Political Economy, Bd. LXXII, 1964, S.486ff.

BUCHANAN, J.M., Public Debt, Cost Theory and Fiscal Illusion, in: J.M. Ferguson (Hrsg.): Public Debt and Future Generations, Chapel Hill 1964, S. $150 \mathrm{ff}$.

BUCHANAN, J.M., Concerning Future Generations, in: J.M.Ferguson (Hrsg.): Public Debt and Future Generations, Chapel Hill 1964, S.55ff.

BUCHANAN, J.M., The Icons of the Public Debt, in: Journal of Finance, Bd. 21, 1966, S. $549 \mathrm{ff}$.

BUCHANAN, J.M., Public Finance in Democratic Process, Chapel Hill 1967.

BUChanAN, J.M. und WAGNER, R.E., Public Debt in a Democratic Society, Washington 1967.

BUCHANAN, J.M., Cost and Choice. An Inquiry in Economic Theory, Chicago 1969.

BUCHANAN, J.M. und FLOWERS, M.R., The Public Finances, 4. Auflage, Homewood 1975. 
CLARK, J.M., The Cost of the World War to the American People, New Haven 1931.

COHEN, J., Distributional Effects of the Federal Debt, in: Journal of Finance, Vol. VI, 1951, S.267ff.

DEUTSCHE BUNDESBANK (Hrsg.), Entwicklung des Geldvermögens und der Verpflichtungen von 1950 bis 1971, in: Monatsberichte der Deutschen Bundesbank, 25. Jg., 1973, Heft 3, S.24ff.

DEUTSCHE BUNDESBANK (Hrsg.), Geschäftsbericht der Deutschen Bundesbank für das Jahr 1975.

DIECKHEUER, G., Eine dynamische Analyse des "Crowding-out"-Effektes zusätzlicher Staatsausgaben, in: Finanzarchiv, NF Bd. 33, 1974/75, S.387ff.

DIEKWISCH, S., Das Zeitmoment in der antizyklinschen Finanzpolitik der Bundes republik, Diss. Freiburg 1974.

DONNER, O., Die Grenzen der Staatsverschuldung, in: Weltwirtschaftliches Archiv, Bd. 56, 1942.

DORN, D., Die Theorie der Staatswirtschaft in der englischen Klassik, Diss. Nürnberg-Erlangen 1968.

DREISSIG, W., Zur Entwicklung der öffentlichen Finanzwirtschaft seit dem Jahr 1950, in: Deutsche Bundesbank (Hrsg.): Währung und Wirtschaft in Deutschland 1876-1975, Frankfurt 1976, S.691ff.

DUWENDAG, D., Investitionsdeterminanten im Wohnungs - und Städtebau während der deutschen Nachkriegsperiode, in: Jahrbuch für Sozialwissenschaft, Bd. 18, 1967, S.223ff.

DUWENDAG, D., KETTERER, K.-H., KÖSTERS, W., POHL, R. und SIMMERT, D.B., Geldtheorie und Geldpolitik, Köln 1974.

ECKSteIN, O., Public Finance, 3. Auflage, Englewood Cliffs, 1973.

EHRLICHER, W., Finanzwissenschaft, in: W. Ehrlicher u.a. (Hrsg.):

Kompendium der Volkswirtschaftslehre, Band 2, 4. Auflage, Göttingen 1975 , S.298ff.

EICHNER, A.S. und KRENGEL, J.A., An Essay on Post-Keynesian Theory: A New Paradigma in Economics, in: The Journal of Economic Literature, Bd. 13, 1975, S. 1293ff.

EISNER, G., Kaufkraftübertragungen durch öffentliche Finanzen, Winterthur 1956.

ELLIOTT, J.R., The Burden of the Public Debt, Comment, in: J.M.Ferguson (Hrsg.): Public Debt and Future Generations, Chapel Hill 1964, S.84ff.

ENGELS, W., SABLOTNY, H. und ZICKLER, D., Das Volksvermögen, Frankfurt 1974 . 
FECHER, H., Inzidenzprobleme finanzpolitischer Mittel zur Vermögensumteilung, in: W. Albers (Hrsg.): Öffentliche Finanzwirtschaft und Verteilung I, Schriften des Vereins für Socialpolitik, NF Bd. 75, I, Berlin 1974, S.95ff.

FELS, G., Fiskalistische oder monetaristische Konjunkturstabilisierung? in:

B. Gahlen und H.K.Schneider (Hrsg.): Grundfragen der Stabilitätspolitik, Tübingen 1974, S.199ff.

FERGUSON, J.M., Introduction, in: J.M.Ferguson (Hrsg.): Public Debt and Future Generations, Chapel Hill 1964, S.3ff.

FOX, K. -P., Die Berücksichtigung von Preisniveausteigerungen bei der $\mathrm{Be}-$ urteilung konjunktureller Wirkungen öfentlicher Haushalte, in: Finanzarchiv, NF Bd. 33, 1974/75, S. $435 \mathrm{ff}$.

FREY, R. L., Finanzpolitik und Verteilungsgerechtigkeit, in: Finanzarchiv, NF Bd. 31, 1972, S.1ff.

GANDENBERGER, O.,' Ist es möglich, durch die Aufnahme von öffentlichem Kredit die Last einer öffentlichen Ausgabe in die Zukunft zu verschieben? in: Jahrbuch für Sozialwissenschaft, Bd. 21, 1970, S.87ff.

GANDENBERGER, O., Öffentlicher Kredit und Einkommensverteilung, in: Finanzarchiv, NF Bd. 29, 1970, S.1ff.

GANDENBERGER, O., Zur Rationalität der öffentlichen Kreditnahme. Verhaltenshypothesen und normative Konsequenzen, in: Finanzarchiv, NF Bd. 30, 1971, S. 369ff.

GANDENBERGER, O., Intertemporale Verteilungswirkungen der Staatsverschuldung, in: H.Haller und W.Albers (Hrsg.): Probleme der Staatsverschuldung. Schriften des Vereins für Socialpolitik, NF Bd. 61, Berlin 1972 , S. 189ff.

GANDENBERGER, O., Die Wirkung des öffentlichen Kredits auf die Einkommensverteilung, in: Das Wirtschaftsstudium, 3. Jg., 1974, S. 383ff.

GAUDE, B., Die Mechanismen der Zentralbankgeldschöpfung und ihre Kontrollierbarkeit durch die Zentralbank, Berlin 1969.

GILLESPIE, W.I., Effect of Public Expenditures on the Distribution, in: R.A. Musgrave (Hrsg.): Essays in Fiscal Federalism, Washington 1965, S. $157 \mathrm{ff}$.

GRUBER, U., Einkommensverteilung. Wirtschaft und Gerechtigkeit, München/ Wien 1974.

H ÄUSSLER, G., Die Problematik der Last der öffentlichen Schuld, Diss. Graz 1961 .

HAKE, W., Umverteilungseffekte des Budgets, Göttingen 1972. 
HALLER, H., Zur Problematik der Kreditfinanzierung öffentlicher Ausgaben, in: Finanzarchiv, NF Bd. 19, 1958/59, S. 72ff.

HALLER, H., Finanzpolitik, 5. verb. Auflage, Tübingen 1972.

HALLER, H., Neue Motivation der Staatsverschuldung, in: H.Haller und H.C. Recktenwald (Hrsg.): Finanz- und Geldpolitik im Umbruch, Mainz 1969, S. 301ff.

HANSCHEL, J., Kreislauftheorie und Staatshaushalt, Zürich 1967.

HANSEN, A., Die Einkommens- und Vermögensverteilung in der Bundesrepublik Deutschland aus statistischer Sicht, in: W.Albers (Hrsg.): Öffentliche Finanzwirtschaft und Verteilung I, Schriften des Vereins für Socialpolitik, NF Bd. 75/I, Berlin 1974, S. $21 \mathrm{ff}$.

HANSMEYER, K. -H., La dette publique comme moyen de reporter des charges sur les générations futures, in: Revue de Science Financière, Bd. 55, 1963 , S. 543ff.

HANSMEYER, K. -H., Der öffentliche Kredit, 2. Auflage, 1970.

HANSMEYER, K. -H. und MACKSCHEIDT, K., Finanzpsychologie, in: F. Neumark (Hrsg.): Handbuch der Finanzwissenschaft, 3. Auflage, Tübingen 1976, Teillieferung 7-9, S.553ff.

HARRIS, S.E., The National Debt and the New Economics, New York/London 1947.

HAUSER, R., Geldwertgesicherte Anlagemöglichkeiten als neues sozialpolitisches Instrument, in: Finanzarchiv, NF Bd. 34, 1976, S.456ff.

HEAD, J.G., The Theory of Debt Incidence, in: Rivista di Diritto Finanziario e Scienza delle Finanze, Bd. 26, 1967, S. 175ff.

HECK, J., Die Problematik der Staatsschuldentilgung in der Theorie vom Merkantilismus bis zur Gegenwart, Diss. Frankfurt 1954.

HEDTKAMP, G., Lehrbuch der Finanzwissenschaft, Neuwied und Berlin 1968.

HENKE, K. -D., Probleme der Staatsverschuldung, in: Zeitschrift für Wirtschafts - und Sozialwissenschaften 1973, 93. Jg, S. $51 \mathrm{ff}$.

HENKE, K. -D., Die Verteilung von Gütern und Diensten auf die verschiedenen Bevölkerungsschichten, Schriften der Kommission für wirtschaftlichen und sozialen Wandel 82, Göttingen 1975.

HESSLER, H. -D., Gegenwartsprobleme staatlicher Anleihepolitik, Forschungsberichte des Landes Nordrhein-Westfalen Nr. 1373, Köln-Opladen 1964.

HÖVELMANN, P., Die Verteilungseffekte von Gewinnsteuern in angebots - und nachfragetheoretischen makroökonomischen Modellen, Diss. Freiburg 1973. 
HOF, H. -J. und WAGNER, U., Verteilungswirkungen externer Effekte - dargestellt am Beispiel verfahrensbedingter Umweltbelastungen, in: D.Cassel und H.J. Thieme (Hrsg.): Einkommensverteilung im Systemvergleich, Stuttgart 1976, S.123ff.

JARCHOW, H. -J., Theorie und Politik des Geldes. II. Geldmarkt und geldpolitische Instrumente, Göttingen 1974.

JECK, A., Wer gewinnt, wer verliert bei einer Inflation? in: J.Schlemmer (Hrsg.): Enteignung durch Inflation, München 1972, S.90ff.

KALDOR, N., Alternative Theories of Distribution, in: The Review of Economic Studies, Bd. 23, 1955/56, wiederabgedruckt in: N. Kaldor: Essays on Value and Distribution, London 1960, S.209ff.

KALDOR, N., Marginal Productivity and the Macroeconomic Theories of Distribution. Comment on Samuelson and Modigliani, in: The Review of Economic Studies, Bd. 33, 1966, S.309ff.

KAMP, M.E., SCHÖNEBECK, W. , SMOLINSKI, D. und WEILER, H., Öffentliche Finanzwissenschaft, Köln 1975.

KATH, D., Die Bedeutung des staatlichen Sparens für die Kapitalbildung und das Wirtschaftswachstum, Berlin 1968.

KIEPE, K., Die Thesen James M.Buchanans zur Last der Staatsverschuldung, Diss. Münster 1970.

KITSCHLER, W., Entwicklungslinien der neueren Steuerinzidenzlehre, Diss. Mainz 1965.

KLOTEN, N., Erfolg und Mißerfolg der Stabilisierungspolitik (1969-1974), in: Deutsche Bundesbank (Hrsg.): Währung und Wirtschaft in Deutschland $1876-1975$, Frankfurt 1976, S. 643ff.

KOBLITZ, H.G., Einkommensverteilung und Inflation in kurzfristiger Analyse, Berlin-New York 1971.

KOCH, W.A.S., Die Gläubigerstruktur als Ansatzpunkt für ein staatliches debt management, Meisenheim am Glan 1972.

KOCK, H., Staatsverschuldung, Wachstumszyklen und Einkommensverteilung, in: B.Gahlen (Hrsg.): Wachstumszyklen und Verteilung, Tübingen 1974, S. 262ff.

KÖHLER, C., Probleme der Geldmengensteuerung, Arbeitspapiere des Instituts für Empirische Wirtschaftsforschung, Heft 4, Berlin 1976.

KOELLREUTER, C., Zur Theorie der internationalen Steuerinzidenz. Ein neoklassischer und ein postkeynesianischer Beitrag, Zürich 1973.

KOULIS, J., Wirtschaftliche Grundfragen des Staatskredits, Frankfurt 1948.

KRELLE, W., Die Investitionsfunktion, in: Jahrbücher für Nationalökonomie und Statistik, Bd. 172, 1960, S. $345 \mathrm{ff}$. 
KRELLE, W., Vermögensverteilung: Zahlen, Empfehlungen, Absichtserklärungen, in: Wirtschaftswoche, Band 26, 1972, Heft 39, S. $73 \mathrm{ff}$.

KRELLE, W., Verteilungstheorie, Tübingen 1962.

KROMPHARDT, J., Kapitalbildung in Arbeitnehmerhand und Einkommensverteilung im Gleichgewicht, in: Zeitschrift für die gesamte Staatswissenschaft, Bd. 122, 1966, S.247ff.

KRUPP, H.-J., "Personelle" und "funktionelle" Einkommensverteilung, in: Jahrbücher für Nationalökonomie und Statistik, Bd. 180, 1967, S.1ff.

LERNER, A.P., The Burden of Debt, in: J.M.Ferguson (Hrsg.): Public Debt and Future Generations, Chapel Hill 1964, S.91ff.

LINDVERS, G., Defizitfinanzierung staatlicher Ausgaben und Geldpolitik im Konjunkturablauf. Analyse des Wirkungszusammenhangs auf der Grundlage alternativer monetärer Hypothesen, Diss. Freiburg 1971.

McLURE, G.E., Tax Incidence, Macroeconomic Policy and Absolute Prices, in: Quarterly Journal of Economics, Bd. 84, 1970, S.254ff.

MEADE, J.E., Is the National Debt a Burden? in: J.M.Ferguson: Public Debt and Future Generations, Chapel Hill 1964, S.19ff.

MEISTER, K., Das Problem der Last in der Theorie der öffentlichen Schuld, Diss. Erlangen-Nürnberg 1967.

MIESZKOWSKI, P., Tax Incidence Theory: The Effects of Taxes on the Distribution of Income, in: Journal of Economic Literature, Bd. VII, 1969, S.1103ff.

MILBRADT, G.H., Ziele und Strategien des debt management, Baden-Baden 1975.

MILlER, D.C., Taxes, The Public Debt and Transfers of Income, Urbana 1950 .

MISHAN, E.J., How to Make a Burden of the Public Debt, in: J.M.Ferguson (Hrsg.): Public Debt and Future Generations, Chapel Hill 1964, S.184ff.

MISHAN, E.J., The Burden of the Public Debt: A Rejoinder, in: Journal of Political Economy, Bd. 72, 1964, S. $491 \mathrm{ff}$.

MODIGLIANI, F., Long-run Implications of Alternative Fiscal Policies and the Burden of the National Debt, in: Economic Journal, Bd. 72, 1961, wiederabgedruckt in: J.M.Ferguson (Hrsg.): Public Debt and Future Generations, Chapel Hill 1964, S.107ff.

MOLITOR, B., Öffentliche Armut-privater Reichtum, in: Ordo, Band 24, 1973, S. $141 \mathrm{ff}$.

MOLITOR, B., Unsoziale Staatsverschuldung, in: Verteilungspolitik in Perspektive, Hamburg 1975, S.101ff. 
MORGENSTERN, O., Thirteen Critical Points in Contemporary Economic Theory: An Interpretation, in: Journal of Economic Literature, Band 10, 1972, S. 1163ff.

MÜCKL, W., BAUMGARTEN, P. und SCHMIDT, K. -D., Die Beziehungen zwischen Wirtschaftsstruktur und Einkommensverteilung, Tübingen 1968.

MÜCKL, W., Gleichgewichtswachstum, Einkommens - und Vermögensverteilung, Tübingen 1975.

MÜCKL, W. und HAUSER, R., Die Wirkungen der Inflation auf die Einkommens - und Vermögensverteilung, Schriften der Kommission für wirtschaftlichen und sozialen Wandel 60, Göttingen 1975.

MUSGRAVE, R.A., Theorie der öffentlichen Schuld, in: W.Gerloff und F. Neumark (Hrsg.): Handbuch der Finanzwissenschaft, Bd. 3, 2. Auflage, Tübingen 1958, S. $68 \mathrm{ff}$.

MUSGRAVE, R.A., The Theory of Public Finance, New York 1959.

MUSGRAVE, R.A., Public Debt and Future Generations (Besprechung des Sammelbandes von Ferguson) in: American Economic Review, Bd. 55, 1965, S. $1226 \mathrm{ff}$.

MUSGRAVE, R.A., Finanztheorie, 2. Auflage, Tübingen 1969.

MUSGRAVE, R.A. und MUSGRAVE, P.B., Public Finance in Theory and Practice, New York 1973.

MUSGRAVE, R.A., MUSGRAVE, P.B. und KULlmeR, L., Die öffentlichen Finanzen in Theorie und Praxis Band I, Tübingen 1975.

NEISSER, H., Is the Public Debt a Burden on Future Generations? in: Social Research, Bd. 28, 1961, S.730ff, Wiederabgedruckt in: J.M. Ferguson (Hrsg.): Public Debt and Future Generations, Chapel Hill 1964, S. $145 \mathrm{ff}$.

NEUBAUER, W., Strategien, Techniken und Wirkungen der Geld- und Kreditpolitik, Göttingen 1972.

OBERHAUSER, A., Finanzpolitik und private Vermögensbildung, Köln und Opladen 1963.

OBERHAUSER, A., Staatsschuldentilgung als finanzpolitisches Instrument, in: Finanzarchiv, NF Bd. 24, 1965, S.283ff.

OBERHAUSER, A., Der Investivlohn als Mittel der Verteilungspolitik, in: H. Arndt (Hrsg.): Lohnpolitik und Einkommensverteilung, Schriften des Vereins für Socialpolitik, NF Bd. 51, Berlin 1969, S.273ff.

OBERHAUSER, A., Gewinnorientierte Einkommenspolitik und Staatsverschuldung, in: H.Haller (Hrsg.): Theorie und Praxis des finanzpolitischen Interventionismus, Fritz Neumark zum 70. Geburtstag, Tübingen 1970, S. $529 \mathrm{ff}$. 
OBERHAUSER, A., Entwicklungstendenzen und Bestimmungsfaktoren der Vermögensbildung und Vermögensverteilung in der Bundesrepublik Deutschland, in: G.Bruns und K.Häuser (Hrsg.): Vermögensbildung, Vermögensverteilung und Kapitalmarkt, Frankfurt 1974, S.32ff.

OBERHAUSER, A., Stabilitätspolitik bei steigender Staatsquote, Schriften der Kommission für wirtschaftlichen und sozialen Wandel Nr. 43, Göttingen 1975.

OBERHAUSER, A., Die Liquiditätstheorie des Geldes als Gegenkonzept zum Monetarismus in: Kredit und Kapital, 10. Jg., 1977, S.207ff.

PARRAVICINI, G., Outline of a Monetary Approach to the Theory of Tax Incidence, in: Banca Nazionale del Lavoro, Bd. 10, 1957, S.233ff.

PASINETTI, L.L., Rate of Economic Growth, in: The Review of Economic Studies, Bd. 29, 1962, S. 267ff.

PEACOCK, A.T., The Rehabilitation of Classical Debt Theory, in: Economica, Band 26, 1959, S. $161 \mathrm{ff}$.

PECHMAN, J.A. und OKNER, B.A., Who Bears the Tax Burden? Studies of Government Finance, Brookings Institution, Washington D.C., 1974 .

PEFFEKOVEN, R., Einführung in die Grundbegriffe der Finanzwissenschaft, Darmstadt 1976.

PFLEIDERER, O., Realvermögen und Geldvermögen unter dem Einfluß der Inflation, in: W.Albers (Hrsg.): Öffentliche Finanzwirtschaft und Verteilung II, Schriften des Vereins für Socialpolitik, NF Band 75, II, Berlin 1974, S.9ff.

PHELPS, E.S., Fiscal Neutrality toward Economic Growth, New York 1965.

POHMER, D., Die Staatsverschuldung als Instrument der Verteilungspolitik, in: H.Haller u. W.Albers (Hrsg.): Probleme der Staatsverschuldung, Schriften des Vereins für Socialpolitik, N.F. Band 61, Berlin 1972, S. S. $143 \mathrm{ff}$.

PREISER, E., Investition und Zins, in: E.Preiser: Bildung und Verteilung des Volkseinkommens, 3. Auflage, Göttingen 1963, S.176-196.

PUVIANI, A., Die Illusion in der Finanzwirtschaft, Berlin 1960.

RAHMANN, B., Grundlagen konjunkturbeeinflussender Haushaltspolitik. Ein Beitrag zu einer Theorie der Budgetwirkungen, Berlin 1972.

RASMUSSEN, P.N., On the Inter relations between Growth and the Distribution of Income, in: J. Marchal und B.Ducros (Hrsg.): The Distribution of National Income, London/New York 1968, S. $511 \mathrm{ff}$.

RECKTENWALD, H. -C., Steuerüberwälzungslehre, 2. Auflage, Berlin 1966. 
REINHARDT, H., On the Incidence of Public Debt in: Social Research, Band 12, 1945, S.205ff.

RICARDO, D., Principles of Political Economy and Taxation (hrsgg. von R.M. Hartwell), London 1971

ROLPH, E.R. und BREAK, G.F., Public Finance, New York 1961.

RUGGLES, R., Volkseinkommen und volkswirtschaftliche Gesamtrechnung, Wien/Stuttgart 1952.

SACHVERST ÄNDIGENRAT, Jahresgutachten des Sachverständigenrates 1974.

SCHEELE, E., Theorie der Einkommensverteilung, in: Kompendium der Volkswirtschaftslehre, W.Ehrlicher, I. Esenwein-Rothe, H.Jürgensen und K. Rose (Hrsg.): 5. Auflage, Göttingen 1975, S.297ff.

SCHEELE, E., Einkommensverteilung und Wirtschaftswachstum, Tübingen 1965.

SCHERF, H., Inflation und Einkommensverteilung, in: Weltwirtschaftliches Archiv, Bd. 108, 1968, S.258ff.

SCHILLERT, U., Gewinne als Quelle der Vermögenspolitik? Die Belastbar keit der Unternehmensgewinne durch vermögenspolitische Maßnahmen, Berlin 1976.

SCHLESINGER, H., Fiskalistische oder monetaristische Konjunkturstabilisierung? in: B. Gahlen und H.Schneider (Hrsg.): Grundfragen der Stabilitätspolitik, Tübingen 1974, S.217ff.

SCHLESINGER, H., Vermögensbildung und Vermögensverteilung in der Bundesrepublik Deutschland, in: G.Bruns und K.Häuser (Hrsg.): Vermögensbildung, Vermögens verteilung und Kapitalmarkt, Frankfurt 1974, S. $7 \mathrm{ff}$.

SCHMAH, U., Der Einkommensredistributionseffekt der Anleihefinanzierung bei unterschiedlichen Zeitpräferenzen, in: Finanzarchiv, NF Bd. 26, 1967, S.232ff.

SCHMÖLDERS, G., Grenzen der Staatsverschuldung, in: Zeitschrift für das gesamte Kreditwesen, 20.Jg., 1967, S.1067ff.

SCHMÖLDERS, G., Finanz- und Steuerpsychologie, Reinbek 1970.

SCHMÖLDERS, G., Finanzpolitik, 3. Auflage, Berlin/Heidelberg/New York 1970 .

SCHUMACHER, U., Nationalbudget und öffentlicher Haushalt, Stuttgart 1958.

SCITOVSKY, T., The Burden of the Public Debt: Comment, in: J.M.Ferguson (Hrsg.): Public Debt and Future Generations, Chapel Hill 1964, S.82ff.

SEN, A.K., Neo-Classical and Neo-Keynesian Theories of Distribution, in:

Economic Record, Bd. 39, 1963, S.53ff. 
SHOUP, C.S., Staatsverschuldung und küntige Generationen, in: H.C.Recktenwald (Hrsg.): Finanztheorie, Köln 1969, S.459ff.

SHOUP, C.S., Public Finance, Chicago 1970, 2. Auflage.

SIEBKE, J., Fiskalistische oder monetaristische Konjunkturstabilisierung? in: B. Gahlen und H.Schneider (Hrsg.): Grundfragen der Stabilitätspolitik, Tubingen 1974, S.231ff.

SIEBKE, J. und WILLMS, M., Geldpolitik und Vermögenseffekte, in: S.Klatt und M.Willms (Hrsg.): Strukturwandel und makroökonomische Steuerung, Festschrift für Fritz Voigt, Berlin 1975, S.323ff.

STOBBE, A., Untersuchungen zur makroökonomischen Theorie der Einkommensverteilung, Tübingen 1962 .

TOBIN, J., The Burden of the Public Debt: A Review Article, in: The Journal of Finance, Bd. 20, 1965, S.679ff.

TOBIN, J., Reply, in: Journal of Finance, Bd. 21, 1966, S.547ff.

TOLKEMITT, G., Zur Theorie der langfristigen Wirkungen öffentlicher Verschuldung, Tübingen 1975.

TULLOCK, G., Public Debt - Who Bears the Burden? in: J.M.Fergus on (Hrsg.): Public Debt and Future Generations, Chapel Hill 1964, S.100ff.

VICKREY, V., The Burden of the Public Debt: Comment, in: The American Economic Review, Bd. 51, 1961, S.74ff.

WALTERS, A.A., How to make a Benefit of the Burden of National Debt, in: National Tax Journal, Vol. XX, 1967, S.316ff.

WEISE, H., Probleme der Geld- und Kreditpolitik im Lichte des RadcliffeReports, Tübingen 1964.

WICKE, L., Schuldnergewinne und Gläubigerverluste während einer schleichenden Inflation, in: Zeitschrift für Wirtschafts - und Sozialwissenschaften, 92. Jg., 1972, II. Halbband, S. 563ff.

WICKE, L., Vermögensverteilung und schleichende Inflation, Meisenheim am Glan 1975.

WILLGERODT, H., BARTEL, K. und SCHILLERT, U., Vermögen für alle. Probleme der Bildung, Verteilung und Werterhaltung des Vermögens in der Marktwirtschaft, Düsseldorf-Wien 1971.

WILLMS, M., Der Einsatz der Staatsschuld als geldpolitisches Instrument, in: Kredit und Kapital, 1. Jg., 1968, S.405ff.

WISEMAN, J., The Logic of National Debt Policy, in: J.M.Ferguson (Hrsg.): Public Debt and Future Generations, Chapel Hill 1964, S.176ff.

WISSENSCHAFTLICHER BEIRAT BEIM BUNDESWIRTSCHAFTSMINISTERIUM, Fragen der Staatsverschuldung, Stellungnahme 1968. 
WITTMANN, W., Einführung in die Finanzwissenschaft, I. Teil, Stuttgart 1970. WITTMANN, W., Einführung in die Finanzwissenschaft, III. Teil, Stuttgart 1972 .

WITTMANN, W., Einfuihrung in die Finanzwissenschaft, III. Teil, 2. Auflage, Stuttgart 1976.

WOLF, R., Die Last der Staatsverschuldung unter besonderer Berücksichtigung der Erfahrungen in den USA, Diss. Frankfurt 1955.

ZIERCKE, M., Inflation und Einkommensverteilung in der Bundesrepublik von 1950 bis 1969, in: Jahrbuch für Sozialwissenschaft, Bd. 22, 1971, S.127ff.

ZIMMERMANN, H., Der letzte "klassische" Deckungsgrundsatz, in: Finanzarchiv, NF Bd. 24, 1965, S.70ff.

ZIMMERMANN, H. und HENKE, K. -D., Einführung in die Finanzwissenschaft, München 1975. 


\section{FINANZWISSENSCHAFTLICHE SCHRIFTEN}

Band 1 Prof. Dr. Werner Steden: Finanzpolitik und Einkommensverteilung. Ein Wachstums- und Konjunkturmodell der Bundesrepublik Deutschland. 378 S., 1979

Band 2 Rainer Hagemann: Kommunale Finanzplanung im föderativen Staat. 173 S., 1976.

Band 3 Klaus Scherer: Maßstäbe zur Beurteilung von konjunkturellen Wirkungen des öffentlichen Haushalts. 242 S., 1977.

Band 4 Brita Steinbach: „Formula Flexibility" - Kristische Analyse und Vergleich mit diskretionärer Konjunkturpolitik. 370 S., 1977.

Band 5 Hans-Georg Petersen: Personelle Einkommensbesteuerung und Inflation. Eine theoretisch-empirische Analyse der Lohn- und veranlagten Einkommensteuer in der Bundesrepublik Deutschland. 330 S., 1977.

Band 6 Friedemann Tetsch: Raumwirkungen des Finanzsystems der Bundesrepublik Deutschland. Eine Untersuchung der Auswirkungen der Finanzreform von 1969 auf die Einnahmenposition der untergeordneten Gebietskörperschaften und ihrer regionalpolitischen Zieladäquanz. 384 S., 1978.

Band 7 Wilhelm Pfähler: Normative Theorie der fiskalischen Besteuerung. Ein methodologischer und theoretischer Beitrag zur Integration der normativen Besteuerungstheorie in die Wohlfahrtstheorie. 224 S., 1978.

Band 8 Wolfgang Wiegard: Optimale Schattenpreise und Produktionsprogramme für öffentliche Unternehmen. Second-Best-Modelle im finanzwirtschaftlichen Staatsbereich. 222 S., 1978.

Band 9 Hans P. Fischer: Die Finanzierung des Unweltschutzes im Rahmen einer rationalen Umweltpolitik. 464 S., 1978.

Band 10 Rainer Paulenz: Der Einsatz finanzpolitischer Instrumente in der Forschungsund Entwicklungspolitik. 260 S., 1978.

Band 11 Hans-Joachim Hauser: Verteilungswirkungen der Staatsverschuldung. Eine kreislauftheoretische Inzidenzbetrachtung. 158 S., 1979 
